\title{
Brain
}

\section{ATP1A2- and ATP1A3-associated early profound epileptic encephalopathy and polymicrogyria}

\begin{tabular}{|c|c|}
\hline Journal: & Brain \\
\hline Manuscript ID & BRAIN-2020-01771.R2 \\
\hline Manuscript Type: & Original Article \\
\hline $\begin{array}{r}\text { Date Submitted by the } \\
\text { Author: }\end{array}$ & $\mathrm{n} / \mathrm{a}$ \\
\hline Complete List of Authors: & $\begin{array}{l}\text { Vetro, Annalisa; AOU Meyer, Pediatric Neurology, Neurogenetics and } \\
\text { Neurobiology Unit and Laboratories, Meyer Children's Hospital, University } \\
\text { of Florence, Florence, Italy } \\
\text { Nielsen, Hang; Aarhus Universitet, Department of Biomedicine } \\
\text { Holm, Rikke; Aarhus Universitet, Department of Biomedicine } \\
\text { Hevner, Robert; University of California San Diego Health Sciences, } \\
\text { Department of Pathology, University of California San Diego, San Diego, } \\
\text { CA, USA } \\
\text { Parrini, Elena; AOU Meyer, Pediatric Neurology, Neurogenetics and } \\
\text { Neurobiology Unit and Laboratories, Meyer Children's Hospital, University } \\
\text { of Florence, Florence, Italy } \\
\text { Powis, Zoe; Ambry Genetics Corp } \\
\text { Møller, Rikke; Danish Epilepsy Centre, } \\
\text { Bellan, Cristina; Azienda Ospedaliera Bolognini Seriate, Department of } \\
\text { Neonatal Intensive Care Unit, Bolognini Hospital, ASST-Bergamo Est, } \\
\text { Seriate, Italy } \\
\text { Simonati, Alessandro; University of Verona Policlinico G. B. Rossi, } \\
\text { Neurology (Child Neurology and Neuropathology), Department of } \\
\text { Neuroscience, Biomedicine and Movement, University of Verona, Verona, } \\
\text { Italy } \\
\text { Lesca, Gaëtan; University Hospital Centre Lyon, Department of Medical } \\
\text { Genetics, Member of the ERN EpiCARE, University Hospital of Lyon, Lyon, } \\
\text { France } \\
\text { Helbig, Katherine; Children's Hospital of Philadelphia, Division of } \\
\text { Neurology } \\
\text { Palmer, Elizabeth; Sydney Children's Hospital Randwick, Centre for } \\
\text { Clinical Genetics, Sydney Children's Hospital, Randwick, NSW, Australia; } \\
\text { University of New South Wales, School of Women's and Children's } \\
\text { Health, University of New South Wales, Randwick, NSW, Australia } \\
\text { Mei, Davide; AOU Meyer, Pediatric Neurology, Neurogenetics and } \\
\text { Neurobiology Unit and Laboratories, Meyer Children's Hospital, University } \\
\text { of Florence, Florence, Italy } \\
\text { Ballardini, Elisa; University of Ferrara, Neonatal Intensive Care Unit, } \\
\text { Pediatric Section, Department of Medical Sciences, Ferrara University, } \\
\text { Ferrara, Italy } \\
\text { van Haeringen, Arie ; Leids Universitair Medisch Centrum, Department of } \\
\text { Clinical Genetics } \\
\text { Syrbe, Steffen; University Hospital Heidelberg, Department of General } \\
\text { Paediatrics, Division of Child Neurology and Inherited Metabolic Diseases, }\end{array}$ \\
\hline
\end{tabular}




\begin{tabular}{|c|c|}
\hline & $\begin{array}{l}\text { Centre for Paediatrics and Adolescent Medicine, University Hospital } \\
\text { Heidelberg, Heidelberg, Germany } \\
\text { Leuzzi, Vincenzo; Sapienza University of Rome, Department of Human } \\
\text { Neuroscience, Unit of Child Neurology and Psychiatry, Sapienza } \\
\text { University, Rome, Italy } \\
\text { Cioni, Giovanni; IRCCS Fondazione Stella Maris, Department of } \\
\text { Developmental Neuroscience, IRCCS Fondazione Stella Maris, Pisa, Italy } \\
\text { Curry, Cynthia; University of California San Francisco, Genetic Medicine, } \\
\text { Department of Pediatrics, University of California, San Francisco/Fresno, } \\
\text { CA, USA } \\
\text { Costain, Gregory ; The Hospital for Sick Children, Division of Clinical and } \\
\text { Metabolic Genetics, Department of Pediatrics, The Hospital for Sick } \\
\text { Children, Toronto, Ontario, Canada } \\
\text { Santucci, Margherita; IRCCS, Institute of Neurological Sciences, Bellaria } \\
\text { Hospital, Bologna, Italy, Child Neuropsychiatry Unit, IRCCS, Institute of } \\
\text { Neurological Sciences, Bellaria Hospital, Bologna, Italy; University of } \\
\text { Bologna, DIBINEM } \\
\text { Chong, Karen; Mount Sinai Hospital, University of Toronto, Toronto, } \\
\text { Ontario, Canada, he Prenatal Diagnosis and Medical Genetics Program, } \\
\text { Department of Obstetrics and Gynecology, Mount Sinai Hospital, } \\
\text { University of Toronto, Toronto, Ontario, Canada } \\
\text { Mancini, Grazia; Erasmus MC University Medical Center, Rotterdam, The } \\
\text { Netherlands, Department of Clinical Genetics } \\
\text { Clayton-Smith, Jill; The University of Manchester, Manchester Centre for } \\
\text { Genomic Medicine, University of Manchester, St Mary's Hospital, } \\
\text { Manchester, United Kingdom } \\
\text { Bigoni, Stefania; Ferrara University Hospital, Department of Mother and } \\
\text { Child } \\
\text { Scheffer, Ingrid; University of Melbourne, Austin Health and Royal } \\
\text { Children's Hospital and Florey Neuroscience Institutes, Epilepsy Research } \\
\text { Centre and Departments of Medicine and Pediatrics } \\
\text { Dobyns, William ; University of Minnesota, Department of Pediatrics } \\
\text { (Genetics), University of Minnesota, Minneapolis, USA } \\
\text { Vilsen, Bente ; Aarhus Universitet, Department of Biomedicine } \\
\text { Guerrini, Renzo; AOU Meyer, Pediatric Neurology, Neurogenetics and } \\
\text { Neurobiology Unit and Laboratories, Meyer Children's Hospital, University } \\
\text { of Florence, Florence, Italy }\end{array}$ \\
\hline Methodology: & GENETICS, GENOMICS \& EPIGENETICS \\
\hline Subject area: & Genetics, Epilepsy \\
\hline
\end{tabular}

\section{SCHOLARONE ${ }^{\text {m }}$ Manuscripts}




\section{$A T P 1 A 2-$ and $A T P 1 A 3-$ associated early profound epileptic encephalopathy and polymicrogyria}

Annalisa Vetro ${ }^{1}$, Hang N. Nielsen ${ }^{2}$, Rikke Holm ${ }^{2}$, Robert F. Hevner ${ }^{3}$, Elena Parrini ${ }^{1}$, Zoe Powis, $\mathrm{MS}^{4}$, Rikke S. Møller ${ }^{5,6}$, Cristina Bellan ${ }^{7}$, Alessandro Simonati ${ }^{8}$, Gaétan Lesca ${ }^{9}$, Katherine L. Helbig ${ }^{10}$, Elizabeth E. Palmer ${ }^{11,12}$, Davide Mei $^{1}$, Elisa Ballardini ${ }^{13}$, Arie Van Haeringen ${ }^{14}$, Steffen Syrbe ${ }^{15}$, Vincenzo Leuzzi ${ }^{16}$, Giovanni Cioni ${ }^{17}$, Cynthia J. Curry ${ }^{18}$, Gregory Costain ${ }^{19}$, Margherita Santucci ${ }^{20,21}$, Karen Chong ${ }^{22}$, Grazia M. S. Mancini ${ }^{23}$, Jill Clayton-Smith ${ }^{24}$, ATP1A2/A3-collaborators ${ }^{\S}$, Stefania Bigoni ${ }^{25}$, Ingrid E. Scheffer ${ }^{26}$, William B. Dobyns ${ }^{27 *}$, Bente Vilsen ${ }^{2 *}$, and Renzo Guerrini ${ }^{1 *}$

1. Pediatric Neurology, Neurogenetics and Neurobiology Unit and Laboratories, Meyer Children's Hospital, University of Florence, Florence, Italy

2. Department of Biomedicine, Aarhus University, DK-8000, Aarhus C, Denmark

3. Department of Pathology, University of California San Diego, San Diego, CA, USA

4. Ambry Genetics, Aliso Viejo, CA, USA

5. Department of Epilepsy Genetics and Personalized Medicine Danish Epilepsy Centre, Filadelfia, Denmark

6. Department of Regional Health Services, University of Southern Denmark, Odense, Denmark

7. Department of Neonatal Intensive Care Unit, Bolognini Hospital, ASST-Bergamo Est, Seriate, Italy

8. Neurology (Child Neurology and Neuropathology), Department of Neuroscience, Biomedicine and Movement, University of Verona, Verona, Italy

9. Department of Medical Genetics, Member of the ERN EpiCARE, University Hospital of Lyon, Lyon, France

10. Division of Neurology, Children's Hospital of Philadelphia, Philadelphia, Pennsylvania

11. Centre for Clinical Genetics, Sydney Children's Hospital, Randwick, NSW, Australia

12. School of Women's and Children's Health, University of New South Wales, Randwick, NSW, Australia

13. Neonatal Intensive Care Unit, Pediatric Section, Department of Medical Sciences, Ferrara University, Ferrara, Italy 
14. Department of Clinical Genetics, Leiden University Medical Center, Leiden, Netherlands

15. Division of Pediatric Epileptology, Centre for Paediatrics and Adolescent Medicine, University Hospital Heidelberg, Heidelberg, Germany

16. Department of Human Neuroscience, Unit of Child Neurology and Psychiatry, Sapienza University, Rome, Italy

17. Department of Developmental Neuroscience, IRCCS Fondazione Stella Maris, Pisa, Italy

18. Genetic Medicine, Department of Pediatrics, University of California, San Francisco/Fresno, CA, USA

19. Division of Clinical and Metabolic Genetics, Department of Pediatrics, The Hospital for Sick Children, Toronto, Ontario, Canada

20. Child Neuropsychiatry Unit, IRCCS, Institute of Neurological Sciences, Bellaria Hospital, Bologna, Italy

21. DIBINEM, University of Bologna, Bologna, Italy

22. The Prenatal Diagnosis and Medical Genetics Program, Department of Obstetrics and Gynecology, Mount Sinai Hospital, University of Toronto, Toronto, Ontario, Canada

23. Department of Clinical Genetics, Erasmus MC University Medical Center, Rotterdam, The Netherlands

24. Manchester Centre for Genomic Medicine, University of Manchester, St Mary's Hospital, Manchester, United Kingdom

25. Medical Genetics Unit, Department of Mother and Child, Ferrara University Hospital, Ferrara, Italy

26. University of Melbourne, Austin Health and Royal Children's Hospital, Florey and Murdoch Institutes, Melbourne, Australia

27. Department of Pediatrics (Genetics), University of Minnesota, Minneapolis, USA

$\S$ The list of ATP1A2/A3-collaborators is available in the Appendix 1, with full details in the Supplementary Material

* These authors contributed equally to this work

\section{Corresponding author}

Prof. Renzo Guerrini,

Neuroscience Department, Children's Hospital Anna Meyer-University of Florence, Viale

Pieraccini 24, 50139 Florence, Italy; telephone: +39 0555662573, e-mail: r.guerrini@meyer.it 
Correspondence for Neuropathology may be addressed to:

Robert F. Hevner,

Department of Pathology, University of California San Diego, San Diego, CA, USA:

rhevner@ucsd.edu

Running title: $\mathrm{Na}^{+} \mathrm{K}^{+}$-pump dysfunction and encephalopathy 


\section{Abstract}

Constitutional heterozygous mutations of $A T P 1 A 2$ and $A T P 1 A 3$, encoding for two distinct isoforms of the $\mathrm{Na}^{+}, \mathrm{K}^{+}$-ATPase (NKA) alpha-subunit, have been associated with familial hemiplegic migraine (ATP1A2), alternating hemiplegia of childhood (ATP1A2/A3), rapid-onset dystonia-parkinsonism, cerebellar ataxia-areflexia-progressive optic atrophy, and relapsing encephalopathy with cerebellar ataxia (all ATP1A3). A few reports have described single individuals with heterozygous mutations of $A T P 1 A 2 / A 3$ associated with severe childhood epilepsies. Early lethal hydrops fetalis, arthrogryposis, microcephaly, and polymicrogyria have been associated with homozygous truncating mutations in ATP1A2. We investigated the genetic causes of developmental and epileptic encephalopathies variably associated with malformations of cortical development in a large cohort and identified 22 patients with de novo or inherited heterozygous ATP1A2/A3 mutations. We characterized clinical, neuroimaging and neuropathological findings, performed in-silico and in-vitro assays of the mutations' effects on the NKA-pump function, and studied genotype-phenotype correlations. Twenty-two patients harboured 19 distinct heterozygous mutations of ATP1A2 (six patients, five mutations) and ATP1A3 (16 patients, 14 mutations, including a mosaic individual). Polymicrogyria occurred in ten $(45 \%)$ patients, showing a mainly bilateral perisylvian pattern. Most patients manifested early, often neonatal, onset seizures with a multifocal or migrating pattern. A distinctive, 'profound' phenotype, featuring polymicrogyria or progressive brain atrophy and epilepsy, resulted in early lethality in seven patients (32\%). In-silico evaluation predicted all mutations to be detrimental. We tested 14 mutations in transfected COS-1 cells and demonstrated impaired NKA-pump activity, consistent with severe loss of function. Genotype-phenotype analysis suggests a link between the most severe phenotypes and lack of COS-1 cell-survival, and also reveals a wide continuum of severity distributed across mutations that variably impair NKApump activity. We performed neuropathological analysis of the whole brain in two individuals with polymicrogyria respectively related to a heterozygous ATP1A3 mutation and a homozygous ATP1A2 mutation and found close similarities with findings suggesting a mainly neural pathogenesis, compounded by vascular and leptomeningeal abnormalities. Combining our report with all prior reports, we estimate that about 5\% of mutations in ATP1A2 and 12\% in $A T P 1 A 3$ can be associated with the severe and novel phenotypes that we describe here. Notably, a few of these mutations were associated with more than one phenotype. These findings assign novel, 'profound' and early lethal phenotypes of developmental and epileptic encephalopathies and polymicrogyria to the phenotypic spectrum associated with 
heterozygous- $A T P 1 A 2 / A 3$ mutations and indicate that severely impaired NKA pump function can disrupt brain morphogenesis.

Keywords: ATP1A2, ATP1A3, developmental and epileptic encephalopathy; polymicrogyria; $\mathrm{Na}^{+} / \mathrm{K}^{+}$-ATPase pump 


\section{INTRODUCTION}

The $\mathrm{Na}^{+} / \mathrm{K}^{+}$-ATPase (NKA) ion pump is a ubiquitous transmembrane enzyme responsible for active exchange of $\mathrm{Na}^{+}$and $\mathrm{K}^{+}$ions across the plasma membranes of higher eukaryotic cells (Kaplan, 2002). In neural tissue, this process is pivotal for maintaining the resting membrane potential, excitability, and secondary active transport that involves $\mathrm{Na}^{+} / \mathrm{H}^{+}$and $\mathrm{Na}^{+} / \mathrm{Ca}^{2+}$ exchange, $\mathrm{K}^{+} / \mathrm{Cl}^{-}$cotransport, and $\mathrm{Na}^{+}$-dependent neurotransmitter uptake (Friedrich et al., 2016). The NKA pump transports three $\mathrm{Na}^{+}$ions out of the cell and two $\mathrm{K}^{+}$ions into the cell for each ATP hydrolysed, undergoing large conformational changes between two principal conformations (E1 and E2) and their phosphorylated intermediates $\left(\mathrm{E}_{1} \mathrm{P}\right.$ and $\left.\mathrm{E}_{2} \mathrm{P}\right)$ (Kaplan, 2002; Friedrich et al., 2016; Holm et al., 2016). It is composed of a large catalytic $\alpha$ subunit and smaller $\beta$ and $\gamma$ subunits. The $\alpha$-subunit contains binding sites for three $\mathrm{Na}^{+}$ions in the $\mathrm{E} 1$ conformation or two $\mathrm{K}^{+}$ions in the $\mathrm{E} 2$ conformation, as well as a catalytic site that binds ATP and phosphate. The $\beta$-subunit is required for protein folding and targeting the $\alpha$-subunit to the plasma membrane, while both $\beta$ and $\gamma$ subunits act as fine modulators of ion affinity in different tissues (Friedrich et al., 2016).

The four known human isoforms of the $\alpha$-subunit ( $\alpha 1-4)$ encoded by four paralogous genes (ATP1A1-4) share $84-91 \%$ of the amino acid sequence and have developmental and tissue expression specificity. The isoforms differ in affinity for $\mathrm{Na}^{+}, \mathrm{K}^{+}$and ATP, and have different enzyme kinetics (Blanco, 2005). The $\alpha 2$ - and $\alpha 3$-isoforms, encoded by ATP1A2 and ATP1A3, are predominantly expressed in CNS. The expression of both isoforms is primarily neuronal during embryonic development. In the adult brain, $\alpha 3$ maintains its neuronal expression while $\alpha 2$ is primarily expressed in glial cells (McGrail et al., 1991; Moseley et al., 2003). Multiple isoforms of $\beta$ and $\gamma$ regulatory subunits also exist, with different tissue distribution and functional effects.

Constitutional heterozygous mutations of $A T P 1 A 2 / A 3$ have been associated with several autosomal dominant neurological disorders with limited overlap. These include familial hemiplegic migraine (FHM; ATP1A2 - MIM\#602481); rapid-onset dystonia-parkinsonism (RDP; ATP1A3 - MIM\#128235); alternating hemiplegia of childhood [AHC; ATP1A3-MIM \#614820 and a single family with an ATP1A2 mutation (Bassi et al., 2004; Swoboda et al., 2004)]; cerebellar ataxia-areflexia-progressive optic atrophy (CAPOS; ATP1A3 MIM\#601338); and relapsing encephalopathy with cerebellar ataxia [RECA; ATP1A3 - (Dard et al., 2015)]. Epilepsy and intellectual disability may co-occur with AHC and FHM, and severe epilepsies have been described in rare patients with ATP1A2 or ATP1A3 mutations (Paciorkowski et al., 2015; Liu et al., 2018). Early lethal hydrops fetalis, intrauterine growth 
restriction, arthrogryposis, microcephaly, polymicrogyria, and lack of respiratory drive have been associated with homozygous truncating mutations in ATP1A2 (Chatron et al., 2019; Monteiro et al., 2020).

In this study, we describe a spectrum of severe neurodevelopmental phenotypes associated with heterozygous mutations of either $A T P 1 A 2$ or $A T P 1 A 3$ that may include early lethality, most often occurring during status epilepticus, microcephaly and the cortical malformation polymicrogyria as well as progressive brain atrophy. Combined with prior reports of FHM, RDP, AHC, CAPOS and RECA, our data define a wide spectrum of disorders that we designate "ATP1A2/A3-opathies". We explore the detrimental effects of causative mutations on NKA pump function using several different experimental paradigms and show that the most severe phenotypes are caused by mutations that are lethal in mammalian cell culture (COS-1 cells). We also demonstrate that neuropathological features of polymicrogyria associated with heterozygous ATP1A3 and biallelic ATP1A2 mutations are similar and support a mainly neural pathogenesis, compounded by vascular abnormalities.

\section{MATERIALS AND METHODS}

\section{Patients}

Our initial discovery cohort consisted of 2800 individuals referred to the Neuroscience and Neurogenetics Department of the Meyer Children's Hospital to investigate the genetic causes of developmental and epileptic encephalopathies (DEEs, defined according to Scheffer et al, 2017). Within this cohort, we identified de novo and inherited heterozygous mutations of ATP1A2 and ATP1A3 in nine patients (Pts 3, 6, 9, 11, 12, 14-16, and 20). Alerted by this association, we identified another 13 patients with similar features through international collaborations and by interrogating the DECIPHER database (http://decipher.sanger.ac.uk) (Pts $1,2,4,5,7,8,10,13,17,18,19,21$ and 22). We obtained written informed consent from all participants or their legal guardians according to local requirements. The study was approved by the Pediatric Ethics Committee of the Tuscany Region (grant 602531). We reviewed medical records, ECG, EEGs, and brain MRI scans. Seizure types were classified following ILAE criteria (Scheffer et al., 2017) whenever applicable and used more descriptive terms when seizure phenomenology could not be easily classified using classification terminology. We submitted all novel mutations to DECIPHER. One child with a biallelic mutation of ATP1A2 (Pt23) was described clinically in a prior report (Monteiro et al., 2020). Here we report brain autopsy findings and compare them to another child in this cohort. 
Detailed materials and methods for MRI investigations, brain neuropathology in Patients 11 and 23, genetic analysis, homology modeling and structural analysis, and functional characterization of $A T P 1 A 2$ and $A T P 1 A 3$ heterozygous mutations are reported in the Supplementary Material.

\section{RESULTS}

\section{Clinical description}

Clinical and genetic data for 22 patients (11 males/11 females) with 19 heterozygous mutations in ATP1A2 (N=6, Pts 1-6) or ATP1A3 (N=16, Pts 7-22) are summarized in Tables 1 and 2. Data for Patient 23, carrying a biallelic ATP1A2 mutation and not considered in the clinical study, is also included in the Tables.

Epilepsy was reported in 21/22 individuals, with seizure onset during the neonatal period in 11 individuals and during infancy or childhood in the remaining nine. Refractory status epilepticus had occurred in 10 patients, in five of whom as the presenting type of seizure in the neonatal period (Pts 3, 4, 7, 11, and 13). Most had multiple seizure types, with multifocal $(\mathrm{N}=9)$ and focal seizures $(\mathrm{N}=7)$ being most frequent. The multifocal seizure pattern observed in these patients corresponded to the epilepsy of infancy with migrating focal seizures (EIMFS) syndrome (Burgess et al., 2019). Recurrent, prolonged, seizure-related apneic episodes, accompanied by intense cyanosis, were a prominent feature in eight patients ( $\mathrm{Pts} 3,7,11,12$, $16,17,20,21)$. In two additional neonates, apneic episodes were attributed to central apnea (Pts 14 and 18). A burst suppression EEG pattern was documented in two children (Pts 3 and 11). Multiple antiseizure medications were used both in the acute treatment of status epilepticus/prolonged seizures and as chronic treatments, with no evidence of any drug being more effective than any other or causing seizure worsening.

Survival was often short, as eight children died due to complications of status epilepticus (Pts $3,4,7,10,11,17,19)$, or intercurrent respiratory infections (Pt 18); seven of these children had died between ten days and 30 months of life (Pts 3, 4, 7, 11, 17-19) and one at 14 years (Pt 10). Overall, four children's demise happened within the first two months of life (Pts 4, 7, 17 and 19), too early to detect progressive worsening, which was instead apparent in the remaining four who died at a later age. 
ECG data, available for 19 children (ATP1A2: Pts 2-4, 6; ATP1A3: Pts 7-14, 16-22) were unremarkable in all, except Patient 13 in whom a short QT interval with unusual ST segment was reported, which had remained asymptomatic at age 3 years (Table 1).

Severe congenital or postnatal (usually by age two years) microcephaly was observed in 10/22 patients. Global developmental delay and intellectual disability was documented in 18/22 patients, including all who survived long enough to be assessed. The disability was rated as severe to profound in 14 , moderate in three, and mild in 1/18 patients. Severe axial hypotonia was reported in 14 individuals including three with dyskinetic quadriparesis (Pts 2, 10, 18). Attacks of AH were observed in only ene two children thild (Pts 16 and 20), both having polymicrogyria. Additional clinical findings in this cohort included Pierre-Robin sequence (micrognathia with cleft palate, Pt11), optic nerve atrophy (Pt10), and hypogenetic lung syndrome (Pt14).

\section{Brain imaging}

All 22 patients with ATP1A2/A3 heterozygous mutations had at least one brain MRI scan and six were scanned more than once at greater than 1-year intervals. The main finding, observed in ten patients (45\%), was polymicrogyria (nine ATP1A3 mutations: Pts 7, 11, 13-16, 17, 19 and 20; one ATP1A2 mutation: Pt6), which was perisylvian predominant, bilateral in nine patients and unilateral in one (Pt15), inconstantly involving other areas (Fig. 1). Perisylvian involvement was accompanied in all by severe disruption of the sulcal pattern, with a verticallyoriented and extended Sylvian fissure. Associated findings included microcephaly in 7/10 and thick corpus callosum in 4/10 patients (Table 1, Fig. 1). Two subjects with polymicrogyria had repeated brain MRI studies that showed no signs of progressive changes.

Severe and progressive brain atrophy was instead documented in two patients, without polymicrogyria (Pts 2 and 8, Supplementary Fig. 1). In the ten remaining patients, brain MRI was normal or showed mild atrophic changes, and remained so in the two who were scanned twice (Pts 9 and 10).

\section{Neuropathological brain examination}

In Patient 11, with a heterozygous ATP1A3 mutation (A3-K764del), and in Patient 23, with a homozygous ATP1A2 frameshift mutation (A2-R279G*4), we performed post-mortem neuropathological examination and compared findings (Fig. 2 and 3). 
The brain of Patient 11 appeared small for the age of four postnatal months (brain weight not available). The surface gyri were abnormal, mostly small and irregular, but with apparent pachygyria of pericentral cortex bilaterally, and bilateral widening of the central sulcus (Fig. 2A-C). In slices, the corpus callosum was present but small at the genu and was truncated with lack of midline crossing at the level of the posterior body and splenium, thus forming Probst bundles (Fig. 2G). The regions of surface pachygyria showed abnormally thick cortical gray matter (Fig. 2D,E). Hemispheric white matter was also reduced. The basal ganglia and thalami appeared relatively spared, with almost normal size. The cerebellar folia showed no obvious defects. The cerebellar dentate nuclei and the inferior olives had simplified morphology, and no arcuate nuclei were detected. Histology revealed that the macroscopically pachygyric cortex was thick, disorganized, and partially covered by rinds of leptomeningeal glioneuronal heterotopia (Fig. 2F,G). Histology also demonstrated that the macroscopic appearance of pachygyria actually corresponded to disorganized layers with some features of classic fourlayered polymicrogyria (arrowheads, Fig. 2F,G); the pachygyric appearance was conferred in part by the rind of leptomeningeal heterotopia. Some areas exhibited more definitive features of polymicrogyria (arrows, Fig. 2F,G), while other areas showed essentially normal cortical lamination. Overall, these findings indicated a variable malformation of cortical development affecting central regions more severely that was also associated with malformations of brainstem and cerebellar nuclei.

The brain of Patient 23 was very small for the age of 33 gestational weeks ( $91.5 \mathrm{~g}$; expected 220 g). Surface gyri were difficult to assess due to adherent meninges with subarachnoid hemorrhage (agonal) and congested blood vessels (Fig. 3A,B). Midline section revealed that the corpus callosum was small but present, including the splenium (arrow Fig. 3B). The cerebellum was moderately hypoplastic, with reduced foliation (Fig. 3C). Coronal slices through the hemispheres revealed severe hypoplasia of gray and white structures, as well as moderate enlargement of the posterior lateral ventricles (i.e. colpocephaly: Fig. 3D-F). The cortical gyri were diffusely small with extensive folding, suggestive of polymicrogyria, except for the hippocampus, which appeared relatively normal bilaterally (arrows, Fig. 3E). Histology confirmed diffuse polymicrogyria throughout the cortex except in the hippocampus, which displayed relatively normal lamination (Fig. 3G,H). Leptomeningeal glioneuronal heterotopia were widespread over the external surface of the hemispheres (Fig. 3I). Histology also revealed abundant focal calcifications (or mineralization) involving cerebral gray and white matter, leptomeninges and blood vessels (arrows, Fig. 3G-L). Some blood vessels had abnormally thick 
walls (intimal hyperplasia) as well as mineralizations (Fig. 3L). Also, the cerebellar deep nuclei were dysplastic, and the pyramidal tracts were severely hypoplastic (not shown).

\section{Genetic findings}

We identified 19 heterozygous mutations of ATP1A2 $(\mathrm{N}=5)$ or ATP1A3 $(\mathrm{N}=14$; Table 1) including 15 missense substitutions, three in-frame deletions, and one insertion. All 19 mutations affected highly conserved residues among both orthologs and paralogs of ATP1A2 and ATP1A3 (Table 1, Supplementary Fig. 2) and were interpreted as pathogenic based on both in silico analysis using sequence data and the functional studies described below. Two mutations (A2-G366A and A3-S361P) were recurrent, occurring in two individuals each. The mutations occurred de novo in 20/22 patients and were inherited in two individuals from one family (Pts 14-15: A3-D887Y). The latter was inherited across three generations from a reportedly healthy man to his daughter (Pt15) and grandson (Pt14). None of the mutations were present in either GnomAD or our internal dataset.

Analysis of the variant allele frequency (VAF) showed that 21/22 mutations were constitutional and one mosaic with VAF $26.2 \%$. Eleven heterozygous mutations were novel (ATP1A2 N=2; ATP1A3 N=9; Table 1). Of the eight mutations previously reported, three were associated with FHM (Pt6: A2-R908Q) or AHC (Pt10: A3-D609Y; Pt13: A3-D801N) (De Vries et al., 2007; Heinzen et al., 2012; Rosewich et al., 2012; Marzin et al., 2018). The mosaic mutations found in our series (Pt21: A3-F857del) was reported as a constitutional mutation with no clinical details in one individual (Retterer et al., 2016). Four additional mutations found in Patients 1, 3, 16 and 22 reported here had been mentioned in previous series within which these patients had been included (Panagiotakaki et al., 2015; Parrini et al., 2017; Ueda et al., 2018; Burgess et al., 2019). In subjects investigated by WES or WGS, we excluded additional potentially pathogenic mutations.

No additional potentially pathogenic mutations were identified in the 20 patients with available

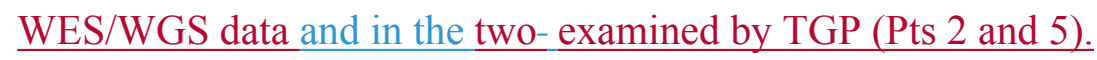

\section{Homology modeling and structural analysis}

Of the 19 mutations in our cohort, eight ATP1A3 mutations, but only two ATP1A2 mutations, affected the transmembrane helices M3 (A3-L292R, A2-I293M), M4 (A3-G316V, A2-C341F), M5 (A3-K764del, A3-P775R), M6 (A3-D801N), M7 (A3-F857del), M8 (A3-L924P), and M10 
(A3-D992dup) (Fig. 4 and Supplementary Fig. 3). The predicted structural impacts of all mutations in our cohort are detailed in Supplementary Table 1 and illustrated in Supplementary Fig. 4-10. A3-D801N has previously been shown to impair $\mathrm{Na}^{+}$and $\mathrm{K}^{+}$transport as $\mathrm{D} 801$ is known to directly bind $\mathrm{Na}^{+}$and $\mathrm{K}^{+}$at both sites I and II (Weigand et al., 2014; Li et al., 2015; Holm et al., 2016). A3-L924P is predicted to break the M8 helix, thereby indirectly disturbing the interaction of the juxtaposed D923 with $\mathrm{Na}^{+}$at site III (Arystarkhova et al., 2019). The mutations A3-L292R, A2-I293M, A3-G316V, A2-C341F, A3-K764del, A3-P775R, A3L924P, and A3-D992dup found in transmembrane helices and A3-P972del in the L8-9 loop connecting the transmembrane helices M8 and M9 are predicted to affect the ion binding sites indirectly. Four mutations affected the P domain, which contains the phosphorylation site (A2G366A, A2-R593Q, A3-S361P, and A3-D609Y). A3-D609Y replaces the aspartate of the conserved TGD motif critical for ATP binding (Holm et al., 2016). The remaining four mutations (A2-R908Q, A3-D887Y, A3-L888P, A3-G893W) affected the L7-8 extracellular loop between transmembrane helices M7 and M8, which interacts with the extracellular domain of the $\beta$-subunit. Hence, these mutations are predicted to interfere with the $\alpha$ - $\beta$ interaction crucial to expression in the plasma membrane. These four mutations were all associated with polymicrogyria. A3-G893W introduces a bulky tryptophan corresponding to the glycine of the SYGQ motif at the interface with the $\beta$-subunit. A3-F857del is likely to affect the interaction between M7 and the transmembrane helix of the $\beta$-subunit. No mutations involved the Nterminal third of the protein including M1-M2 and the A domain.

\section{Functional characterization of $A T P 1 A 2-A 3$ heterozygous mutations}

The primary function of ATP driven NKA pumps is to rapidly exchange three intracellular $\mathrm{Na}^{+}$ ions for two extracellular $\mathrm{K}^{+}$ions. In the presence of ATP and intracellular $\mathrm{Na}^{+}$, the ATP-NKA pump in its E1 state forms a phosphorylated intermediate (E1P) that changes configuration from the E1P to E2P state, exposing $\mathrm{Na}^{+}$to the extracellular space. In the presence of extracellular $\mathrm{K}^{+}$, three $\mathrm{Na}^{+}$are released and two $\mathrm{K}^{+}$bound (Fig. 5D). The phosphorylated intermediate (E2P) is hydrolyzed to the E2 state which equilibrates to the E1 state in the presence of intracellular ATP, delivering two $\mathrm{K}^{+}$to the intracellular space.

Among the Cos- 1 cell lines transfected with the 14 mutations we tested, only three ATP1A2 and two ATP1A3 mutants retained the 5-10\% wild type NKA pump activity required for cell survival under ouabain selection (Holm et al., 2016). The remaining two ATP1A2 and seven ATP1A3 mutants could not support cell growth in stable transfections, indicating that they lacked NKA pump activity or were not expressed in the plasma membrane (Table 2 and 
Supplementary Table 1). Hence, we analysed these mutants by transient transfection into COS1 cells using siRNA to knock down the endogenous NKA pump, as described previously (Nielsen et al., 2019).

Among the five stably expressed mutants retaining transport activity (cell survival), only A2I293M and A2-R593Q showed phosphorylation levels $>50 \%$ wild-type, whereas levels of A2R908Q, A3-P972del, and A3-D887Y were 26-35\% wild-type (Fig. 5A, Table 2). However, the turnover rate (rate of Pi liberation per active site in the presence of both $\mathrm{Na}^{+}$and $\mathrm{K}^{+}$) was markedly reduced for A2-I293M (27\% wild-type) and A2-R593Q (42\% wild-type) (Fig. 5B, Table 2). Hence, the NKA pump activity calculated by multiplying the phosphorylation level by the turnover rate was markedly reduced for all five mutants (17-36\% wild type), thus accounting for the pathogenic effects of all five stably expressed mutations (Table 2).

Among the nine remaining mutants that could only be transiently expressed because of lack of NKA pump activity (Fig. 5C), the phosphorylation levels of A2-G366A (61\%), A3-L292R (61\%), and A3-G316V (70\%) indicated that they were expressed in the plasma membrane and retained the ability to bind $\mathrm{Na}^{+}$and ATP, despite their inability to undergo the complete transport cycle.

To explore the $\mathrm{Na}^{+}$- and $\mathrm{K}^{+}$-binding properties of the mutants, we took advantage of the $\mathrm{Na}^{+}$and $\mathrm{K}^{+}$-dependence of NKA phosphorylation and pump activity, which could be studied for the stably as well as the transiently expressed mutants showing robust phosphorylation. The effects of mutations on the E1-E2 and E1P-E2P conformational changes (cf. reaction cycle in Fig. 5D) are summarized in Fig. 6A,B, Supplementary Fig. 11, and 12, with full text presentation in the Supplementary Materials. The very low or undetectable phosphorylation (expression) levels in the remaining six mutants (A2-C341F, A3-S361P, A3-K764del, A3-P775R, A3-L888P, and A3-P992dup), prevented further study of their phosphorylation properties (Fig. 5C).

In brief, the mutations in the transmembrane helices for which the ion binding properties could be examined (M3: A2-I293M and A3-L292R, M4: A3-G316V, Supplementary Fig. 4 and 9), profoundly interfered with $\mathrm{Na}^{+}$and/or $\mathrm{K}^{+}$binding in various ways. $\mathrm{Na}^{+}$binding was also disturbed by one of the mutations in the P-domain (A2-G366A, Supplementary Fig. 6) and the mutation in the L9-10 loop (A3-P972del, Supplementary Fig. 10). In addition, A2-G366A disturbed $\mathrm{K}^{+}$binding, as the $\mathrm{E} 2 \mathrm{P}$ phosphoenzyme intermediate of this mutant was completely insensitive to $\mathrm{K}^{+}$. The M3 mutant (A2-I293M, Supplementary Fig. 4) had a strong conformational shift in favor of E1/E1P explaining the reduced turnover rate. A similar, but less strong conformational shift was seen for the P-domain mutation near the ATP binding site (A2-R593Q, Supplementary Fig. 7). The remaining mutations in transmembrane helices (A2- 
C341F, A3-K764del, A3-P775R, and A3-D992dup, Supplementary Fig. 5, 9, and 10) resulted in low or undetectable phosphorylation levels, either due to inability to react with $\mathrm{Na}^{+}$and ATP to form a phosphoenzyme, or lack of expression of the mutant in the plasma membrane. The same was the case for the P-domain mutation (A3-S361P) located five residues from the phosphorylated aspartate (Supplementary Fig. 6). Mutations in the L7-8 loop, interacting with the $\beta$-subunit crucial to expression in the plasma membrane (Supplementary Fig. 8), also reduced the phosphorylation level, either partially (A3-D887Y and A2-R908Q) or completely (A3-L888P), most likely due to reduced expression.

In synthesis, in every patient whose mutations were tested at least one of the assays used showed severe impairment, as summarized in Table 2. The 11 mutations not supporting cell growth in culture were associated with the most severe clinical phenotype, while the five mutations supporting cell growth were associated with more variable clinical phenotypes.

\section{DISCUSSION}

Since the first reports of ATP1A2 and ATP1A3 mutations in 2003-2004 (De Fusco et al., 2003; De Carvalho Aguiar et al., 2004) more than 200 mutations ( $=92$ ATP1A2, $\mathrm{N}=136$ ATP1A3) have been identified [Human Gene Mutation Database (HGMD) Professional 2018.3, https://portal.biobase-international.com, last accessed June 2020]. Autosomal dominant disorders associated with $A T P 1 A 3$ mutations have been conceptualized as a phenotypic continuum that includes RDP, AHC, CAPOS, RECA, and DEE (Rosewich et al., 2014; Dard et al., 2015; Paciorkowski et al., 2015). ATP1A2 mutations have been associated with FHM and, in a single family, AHC (Bassi et al., 2004; Swoboda et al., 2004), defining a much narrower and less severe phenotype than ATP1A3 (Fig. 7).

Here we describe 22 individuals with heterozygous $A T P 1 A 2$ or ATP1A3 mutations associated with developmental encephalopathies manifesting most often as early-onset DEE, and associated with frequent early lethality (in 32\%) and polymicrogyria (in 45\%). Clinical and functional findings assign these novel, 'profound' phenotypes to the existing spectrum of disorders associated with heterozygous $A T P 1 A 2 / A 3$ mutations and demonstrate that severely impaired $\mathrm{Na}^{+}, \mathrm{K}^{+}$-pump function can disrupt brain morphogenesis.

Combining our report of 19 mutations (11 novel) with all prior reports, we estimate that about $5 \%$ of mutations in $A T P 1 A 2$ and $12 \%$ in ATP1A3 are associated with the severe and novel phenotypes that we describe in this series. Notably, a few of these mutations were associated with more than one phenotype (Fig. 7). 


\section{Epilepsy}

Epilepsy occurs in about $50 \%$ of patients with AHC and $15-30 \%$ of those with FHM (Deprez et al., 2008; Heinzen et al., 2012; Rosewich et al., 2012; Prontera et al., 2018). In our cohort, which was assembled based on clinical features of developmental encephalopathy, epilepsy occurred in 21 of 22 patients (95\%), with neonatal or early infantile onset in most and episodes of status epilepticus in 10 (45\%). Status epilepticus has been reported to occur in up to $40 \%$ of patients with ATP1A3-AHC (Panagiotakaki et al., 2015) but is a less frequent feature in ATP1A2-FHM (Deprez et al., 2008; Prontera et al., 2018). The occurrence of multiple seizure types, especially multifocal seizures manifested as EIMFS, in most patients indicates widespread epileptogenesis and brain dysfunction. Patients 3 and 4 exhibited a remarkably severe phenotype among those with ATP1A2 mutations reported so far, manifesting neonatal onset super-refractory status epilepticus featuring prolonged apneic episodes. Both patients died within the first year of life without achieving developmental or motor skills. Prolonged seizurerelated apneic episodes were a recurrent feature in five additional individuals, all exhibiting ATP1A3 mutations. In two further patients with ATP1A3 mutations, similar apneic episodes lacked any electrographic correlate and were classified as central apnea. In these patients, structure of the brainstem was intact, suggesting a disturbance of respiratory system regulation. Respiratory disturbances have been described in ATP1A3-AHC (Rosewich et al., 2014). Biallelic ATP1A2 mutations have also been associated with respiratory failure due to absence of spontaneous respiratory effort (Chatron et al., 2019; Monteiro et al., 2020) resembling knockout Atpla 2 or Atpla3 mice, whose defective respiratory rhythm generation was attributed to failed $\mathrm{Cl}^{-}$clearance through the $\mathrm{K}-\mathrm{Cl}$ transporter $(\mathrm{KCC} 2)$ in brainstem postsynaptic neurons (Ikeda et al., 2003, 2017). The KCC2 transporter relies on the $\mathrm{K}^{+}$gradient created by the NKA to pump $\mathrm{Cl}^{-}$out of the cells. The same pathophysiological mechanism might cause the nonepileptic episodic respiratory disturbances in Patients 14 and 18.

Patients with ATP1A3-related disease are at increased risk of exhibiting dynamic ECG abnormalities, a finding substantiated by the multiple cardiac rhythm abnormalities observed the in murine Atpla3 model (Balestrini et al., 2020). However, while sudden seizure-related cardiac death has been induced in murine Atpla3-related disease, premature mortality remained limited to $2 \%$ in the international AHC-ATP1A3 registry cohort over a 20 years period (Rosewich et al., 2017), mainly as a consequence of accidental events or respiratory complications of status epilepticus. In our series, a short QT interval, an ECG abnormality which is potentially predisposing to life threatening arrhythmia, was present in one patient $(\mathrm{Pt}$ 
13) and was the only abnormal finding observed among 19 patients whose ECGs were available for inspection. This patient's mutation, the A3-D801N, a highly recurrent AH mutation, was also present in one patient with $\mathrm{AH}$ and short QT reported by Balestrini et al (Balestrini et al., 2020). The six patients with $A T P 1 A 3$ mutations who died prematurely in our series had normal ECGs. These patients died either as a consequence of an intercurrent respiratory infection or during status epilepticus after progressive worsening of their conditions. These circumstances of death were not consistent with a sudden cardiac event but it cannot be excluded that a compromised cardiac function might have contributed to these patients' vulnerability.

\section{Brain MRI abnormalities and neuropathological findings}

Polymicrogyria, which we observed with consistent features in ten patients $(45 \%)$, is a novel phenotype for heterozygous ATP1A2 and ATP1A3 mutations. Although it is not known how many patients carrying mutations in these genes had good quality MRI imaging, subtle alterations of the cortical folding might have been overlooked in older studies. It is possible that previously reported patients may not have had adequate MRI imaging and thus subtle alterations of the cortical folding might have been overlooked in older studies. In addition, phenotype-guided genetic testing, largely applied in the past, might have prevented novel malformation phenotypes from being found.

In one qualitative MRI study, brain and cerebellar atrophy were reported in up to $50 \%$ of patients with ATP1A3-AHC (Sasaki et al., 2017). Microcephaly with rapidly progressive brain atrophy was described in a single patient with ATP1A3-related DEE (Paciorkowski et al., 2015). Two quantitative MRI studies in small series confirmed reduced total brain volume, with prevalent white matter involvement (Severino et al., 2020) and mild, possibly age related, cerebellar atrophy (Ghusayni et al., 2020).

Polymicrogyria is etiologically heterogeneous and variable in topography (Guerrini and Dobyns, 2014). In this series, the malformation was either limited to the perisylvian cortex or more diffuse but perisylvian predominant. Its characteristics shared similarities with those observed with mutations in several developmental genes (the microcephaly gene WDR62, multiple tubulin genes, the PIK3CA-AKT pathway associated PIK3R2 gene) but recently also associated with the ion channel genes SCN3A (Smith et al., 2018; Zaman et al., 2020) and, in more severe forms, GRIN1 and GRIN2B (Platzer et al., 2017; Fry et al., 2018).

Mutations of $S C N 3 A$ were initially associated with non-malformation epilepsy and DEE (MIM \#617935; MIM \#617938), and more recently with polymicrogyria (Smith et al., 2018; Zaman 
et al., 2020). However, mutations associated with polymicrogyria proved to be gain-of-function resulting in increased non-inactivating persistent $\mathrm{Na}^{+}$currents $\left(\mathrm{I}-\mathrm{Na} \mathrm{P}_{\mathrm{P}}\right)$. Changes in the sodium flux in glia and neurons may reverse $\mathrm{Na}^{+} / \mathrm{Ca}^{2+}$ exchangers triggering calcium waves (Smith et al., 2018; Zaman et al., 2020).

In GRIN1-associated polymicrogyria, gain-of-function mutations causing hyperactivation of NMDA receptors increase intracellular $\mathrm{Ca}^{2+}$, which was predicted to induce polymicrogyria either by causing increased cell death during foetal brain development (excitotoxicity) or by activating signalling pathways such as PI3K-AKT (Fry et al., 2018). A similar mechanism would be at play with GRIN2B mutations (Smith and Walsh, 2020). Altered NMDA receptormediated calcium mobilization disturbs neuronal migration in a mouse model of Zellweger syndrome, a rare metabolic disorder featuring a polymicrogyria-like cortical malformation (Gressens et al., 2000). The altered functioning of the NKA pump provides further evidence that changes in ion homeostasis during embryogenesis may disrupt cortical development (Smith and Walsh, 2020).

One child from a large family segregating FHM (A2-R348P) was reported to have lissencephaly with microcephaly and retinal dysplasia, likely an unrelated phenotype (Pelzer et al., 2017). One of the mutations found in our series has been reported in about $40 \%$ of individuals with AHC (Pt13: A3-D801N) with no brain malformation reported (Rosewich et al., 2012). Different phenotypes associated to the same mutation are not new to ATP1A3. The D923N mutation is recurrent in RDP but has also been observed in one family with four affected individuals with AH (Roubergue et al., 2013). These authors postulated that these clinical syndromes represent the different expressions of the same disorder and that the specific ATP1A3 mutation is not the only determinant of clinical expression, implying that genetic, epigenetic, and environmental factors may all influence clinical expression of ATP1A3 related disease. The A2-R908Q mutation was associated with early onset epileptic encephalopathy in one patient in our series and with familial hemiplegic migraine in multiple unrelated individuals in the literature (De Vries et al., 2007; Roth et al., 2014; Blicher et al., 2016), demonstrating that phenotypic heterogeneity is not limited to ATP1A3 mutations.

We observed variable expressivity for polymicrogyria with one mutation in our series, in which two unrelated individuals carrying the same ATP1A3 mutation (Pts 9 and 20: A3-S361P) had early onset DEE and microcephaly, associated with polymicrogyria and alternating hemiplegia only in one (Pt20). We also observed incomplete penetrance and variable expressivity within a single family in which the proband (Pt14: A3-D887Y) had early onset DEE and bilateral polymicrogyria, his mother (Pt15) had migraine, mild intellectual disability and unilateral 
polymicrogyria, and the maternal grandfather had no reported symptoms and a normal brain MRI despite carrying a germline mutation. Incomplete penetrance and variable expressivity have also been observed with $S C N 3 A$-associated polymicrogyria (Smith et al., 2018). Similarly, GRIN1 mutations even within the same functional domain can result in DEE with normal MRI or polymicrogyria (Fry et al., 2018).

Neuropathological study of the whole brain in Patients 11 (A3-K764del) and 23 (homozygous A2-R279Gfs*4), displayed substantially similar findings. The most obvious similarities were small size of the hemispheres with widespread polymicrogyria covered by extensive leptomeningeal glioneuronal heterotopia, confirmed histologically in both brains. Also, the white matter was atrophic and the corpus callosum small, suggesting that cortical axons were deficient. The most striking difference between the two brains were the abundant mineralizations seen in the brain and blood vessels of Patient 23, which were not observed in Patient 11. Extensive vascular and parenchymal mineralization was also reported in previous clinical and pathological studies of patients with polymicrogyria and biallelic ATP1A2 mutations (Chatron et al., 2019). These findings suggest that ATP1A2 may be important for development and function of both neural and vascular tissue in the brain.

The known expression patterns of $A T P 1 A 2$ and $A T P 1 A 3$ support the microcephaly and polymicrogyria seen in our series (mouse: http://www.informatics.jax.org; human: http://www.hdbr.org/expression). In developing mouse brain, Atpla2 is highly expressed in neural progenitors and meninges, then persists after birth in glial cells (McGrail et al., 1991; Herrera et al., 1994; Moseley et al., 2003). Atpla3 is also expressed in neural progenitors but not in meninges and persists after birth in postnatal neurons (McGrail et al., 1991; Herrera et al., 1994). Thus, both genes could be implicated in direct injury to radial glia and migrating neurons. In sum, the expression patterns of $A T P 1 A 2 / A 3$ in developing brain combined with observations in two brains from our series support a mainly neural (radial glia, progenitors, migrating neurons) pathogenesis that may be compounded by meningeal and vascular abnormalities. No obvious brain anatomical defects have been reported in ATP1A2/A3 mouse models (Ikeda et al., 2003, 2017). However, mouse models are not particularly suitable to unveil abnormal cortical folding and defective cortical lamination might have remained unnoticed as no specific studies seem to have investigated it. 
Different studies have suggested a direct link between the $\mathrm{Na}+, \mathrm{K}+$-ATPase and signal transduction pathways involving, among others, the Src family kinases, and PI3K, which has been implicated in a spectrum of brain malformations. According to these studies, the transmembrane NKA pump would act as a scaffold, tethering signalling proteins together into distinct cellular compartments, thus contributing to the modulation of signal transduction within the cell (Reinhard et al., 2013).

\section{Structural/functional considerations}

A clustering of AHC-associated ATP1A3 mutations in transmembrane domains 2-9 has been previously reported (Rosewich et al., 2014; Holm et al., 2016). Conversely, FHM-associated ATP1A2 mutations and RDP-associated ATP1A3 mutations are scattered throughout the protein (Rosewich et al., 2014; Holm et al., 2016).

In our cohort we observed an enrichment of ATP1A3 mutations in transmembrane domains M3M10 clustered around the ion binding sites (8/14; Supplementary Fig. 3), as previously noted for AHC-associated mutations, suggesting that the most severe ATP1A3-related phenotypes may be associated with this location (Sweadner et al., 2019). There was no clustering distribution of -ATP1A2 mutations in our series, although the A2-G366A mutation, affecting the $\mathrm{P}$ domain, was recurrent in two unrelated patients with super-refractory status epilepticus who died prematurely (Pts 3,4).

Most of the mutations found in our cohort result in severe functional defects based on both disruption of protein structure and biochemical analysis. Many of the mutations replace or insert bulky and/or charged residues such as aromatic amino acids, proline or arginine with smaller or less charged residues, while a few simply delete or duplicate a residue. All 14 of the mutations we tested caused reduced NKA pump activity in the COS-1 cellular model. In nine mutants the NKA pump activity was too low for survival of the transfected COS-1 cells, substantiating the 'profound' phenotypes featuring early death, DEE, progressive brain atrophy and polymicrogyria observed in most patients carrying these mutations (Table 2). The very low pump activity results from severely reduced expression level, inability to form a phosphoenzyme or, for the phosphorylatable mutants A3-L292R, A3-G316V and A2-G366A, a severe reduction in $\mathrm{Na}^{+}$and/or $\mathrm{K}^{+}$affinity. The remaining five mutations caused reduced (17$36 \%$ ) activity that was sufficient for cell growth, attributed to either reduced expression level in the plasma membrane (A2-R908Q, A3-P972del, and A3-D887Y), or an E1P shift of the E1PE2P conformational equilibrium (A2-I293M, A2-R593Q). 
Based on the impaired NKA pump activity observed in the COS-1 cells, where only the mutant NKA is expressed, we classify the mutations in our cohort as loss-of-function, including those mutants for which the impaired pump activity is due to reduced plasma membrane targeting, as previously indicated for A2-R908Q (Tavraz et al., 2009).

Dominant negative effects, which have previously been suggested for A3-D801N (Pt3), A3E815K and A3-G947R (Li et al., 2015) may also be contributing to the severe phenotype in our series. A number of factors argue against haploinsufficiency as the main pathogenetic mechanism, including absence of phenotypic manifestations in carrier parents, similarities of the neuropathological polymicrogyria phenotype with that observed with biallelic mutations [Pt23, (Chatron et al., 2019)] and the nonmalformative phenotypes observed in individuals with large deletions involving ATP1A2/A3 (DECIPHER, https://decipher.sanger.ac.uk/; ClinVar, https://www.ncbi.nlm.nih.gov/clinvar/). Misfolding of the mutant $\alpha$-subunit and allele competition for the $\beta$-subunit might also contribute to deleteriousness of ATP1A3 mutations and their phenotypic heterogeneity (Arystarkhova et al., 2019).

Sweadner et al (Sweadner et al., 2019) suggested that genes that only diverged slightly after past duplications, such as $A T P 1 A 2$ and $A T P 1 A 3$, would be expected to harbor similar pathogenic mutations, but observed that only relatively few mutations affected the corresponding amino acids in these two genes. Our study provides additional examples of mutations affecting homologous residues in ATP1A2 and ATP1A3 (Supplementary Table 1). The ATP1A2 glycine 366 (A2-G366), which is mutated in Patients 3 and 4 (A2-G366A) with early lethal phenotypes, corresponds to the G358 residue in ATP1A3 mutated in a newborn with seizures and progressive brain atrophy who died prematurely (G358V) (Paciorkowski et al., 2015). Different de novo substitutions of A2-G366 have been reported in one patient with refractory epilepsy (A2-G366V) (Liu et al., 2018) and in another with a nonspecific neurodevelopmental disorder (A2-G366C) (McRae et al., 2017). A2-G366, or its equivalent in ATP1A3, is located in the P-domain at the junction between the P1-helix and the P1- $\beta$-strand leading directly to the phosphorylated active site aspartate of the DKTGT motif (Supplementary Fig. 6). The glycine is important for the direction of this $\beta$ strand. In COS- 1 cells transfected with the A3-G366A, the NKA showed a 5-fold reduction in $\mathrm{Na}^{+}$affinity and a highly reduced apparent $\mathrm{K}^{+}$affinity too, with lack of transport activity due to lack of $\mathrm{K}^{+}$-induced dephosphorylation of E2P.

\section{Genotype-phenotype analysis}


The phenotypes observed in our cohort were predominately severe, with short survival (death at $0-3$ years) observed in $7 / 22$ (32\%), severe congenital or postnatal microcephaly in $10 / 22$ (45\%), polymicrogyria in 10/22 (45\%), and severe or profound early developmental delay or intellectual disability in 14/18 (78\%) individuals. We tested 14 mutations from 17 patients and next separated our patient cohort into two groups based on COS-1 cell survival in ouabain selection pressure (Table 2). Group one included 11 patients harbouring 9 different mutations, all Group 1 comprised nine 11 patients whose nine mutations resultinged in lack of cell survival; we observed short early mortality survival in 5/11 (45\%), severe microcephaly in 6/11 (55\%), polymicrogyria in 5/11 (45\%) and severe or profound intellectual disability in $8 / 9$ (89\%) patients. Group 2 included six patients whose mutations were associated with cell survival; we found short survivalearly mortality and severe microcephaly in only $1 / 6$ (17\%) each, polymicrogyria in $3 / 6(50 \%)$ and severe or profound intellectual disability in $2 / 6(33 \%)$ patients. A comparison of the two groups suggests a trend toward more severe phenotype in Group 1, but the differences were not statistically significant due to small numbers (Fisher's Exact Test). Further, all of the phenotypes in both groups were relatively severe, which fits with our finding of severe functional defects in various experimental paradigms for all mutations (Table 2).

For example, seven children (five from Group $1_{2}$ and one from Group 2, and a further patient with the untested A3-G893W) died at 0-3 years with profound encephalopathies classified as DEE. In Ggroup 1, tFwo individuals without polymicrogyria carried the severe A2-G366A mutation, while the remaining four-three all had polymicrogyria with mutations affecting the transmembrane helices (A3-L292R, A3-K764del, and A3-D992dup) and, causing or the SYGQ motif of the L7-8 loop binding the $\beta$-subunit (A3-G893W). The three mutations tested (A3L292R, A3 K764del, A3 D992dup) eaused-severe impairment of NKA pump activity. The, while-A3-L292R alse-caused severe reduction of $\mathrm{Na}^{+}$and $\mathrm{K}^{+}$affinity, compatible with the location of the bulky, positively charged arginine substituent in position to repel $\mathrm{Na}+$ and $\mathrm{K}^{+}$at their binding sites (Supplementary Fig. 9). For A3-K764del and A3-D992dup, phosphorylation and expression were very low or undetectable, most likely caused by misfolding and/or disruption of $\mathrm{Na}^{+}$binding, as these residues are involved in an intricate hydrogen bonding network that is essential for positioning of the transmembrane helices and C-terminus forming the fold for $\mathrm{Na}+$ binding at site III (Supplementary Fig. 10).

The remaining 15 subjects survived infancy although one later died at 14 years. We tested ten mutations from this group, including five (A2-C341F, A3-G316V, A3-S361P, A3-P775R, A3L888P) that resulted in loss of NKA pump activity as indicated by lack of COS-1 cell survival. 
The six children harbouring these five mutations all had severe phenotypes that included intractable epilepsy and severe intellectual disability, variably associated with microcephaly, polymicrogyria and alternating hemiplegia (A3-L888P and A3-S361P), severe hypotonia, and/or progressive brain atrophy (A2-C341F, A3-G316V, A3-S361P). Only the two patients with one child in our series (Pt16) had attacks of alternating hemiplegia in this group (Pts 16 and 20) were the only ones to manifest this disorder in the entire series. However,, although recognition of attacks may have been hampered by severe hypotonia and frequent seizures in the other children. In Pt8:A3-G316V, the low pump activity was associated with low $\mathrm{K}^{+}$ affinity, compatible with insertion of a bulky valine close to the ion binding sites (Supplementary Fig. 9). Phosphorylation and expression were very low or undetectable for A2C341F, A3-S361P, A3-P775R, and A3-L888P. For A3-S361P, the lack of phosphorylation most likely results from redirection of the $\beta$-strand leading to the phosphorylated aspartate by insertion of a proline five residues before the aspartate (Supplementary Fig. 6). For A2-C341F and A3-P775R, the inserted bulky side chains appear to disturb ion binding and helix packing in the membrane, especially when charged arginine is involved (Supplementary Fig. 5 and 9). A3-L888P inserts a proline redirecting the L7-8 loop, predicted to disrupt the interaction between the $\alpha$ - and $\beta$-subunits, which should prevent expression in the plasma membrane (Supplementary Fig. 8).

The remaining five mutations resulted in partially conserved NKA pump activity allowing long term COS-1 cell survival. In the six subjects harbouring these mutations, the phenotypes were of variable severity. Three patients (Pt6: A2-R908Q; Pt14-15: A3-D887Y; Pt18: A3-P972del) had severe phenotypes that overlap with those associated with COS-1 lethal mutations, including severe hypotonia and polymicrogyria. In COS-1 cells, these mutations led to reduced expression in the plasma membrane by interference with the $\alpha-\beta$ interaction (Supplementary Fig. 8) leading to retention of immature protein in the endoplasmic reticulum and/or decreased stability. The ATP1A3 residue homologous to A2-R908 is targeted by an AHC mutation (A3R901T) (Holm et al., 2016) and the ATP1A2 residue homologous to A3-G893 is targeted by a hemiplegic migraine mutation (A2-G900R) (Deprez et al., 2008). The remaining three patients had less severe phenotypes consisting of mild-moderate intellectual disability with mild focal epilepsy (Pt1: A2-I293M; Pt5: A2-R593Q) or unilateral polymicrogyria and migraine (Pt15: A3-D887Y). The less severe phenotypes associated with A2-I293M and A2-R593Q may be related to their near normal expression level, which suggests a relatively stable protein, combined with retention of NKA pump activity, albeit with reduced maximal turnover rate. 
Patient 21 carried the A3-F857del in 26.2\% of WES reads. Mosaicism for ATP1A3 mutations has been reported in a single AHC patient with VAF 44\% and a mild phenotype and in six asymptomatic parents of AHC probands (VAF <18.82\%) (Yang et al., 2019). The phenotype of Patient 21 was severe in spite of the mosaic state of the mutation which is likely to affect the interaction between M7 and the transmembrane helix of the $\beta$ subunit.

\section{Conclusion}

This series links profound early lethal phenotypes with COS-1 lethal mutations, but also reveals a continuum of phenotypic severity, in which both severe and milder phenotypes can be associated with mutations partially sparing NKA pump function, while polymicrogyria is distributed across mutations of variable effect. The molecular diagnostic yield of polymicrogyria is low, with multiple genes accounting for a small number of diagnoses each, and a relevant number of patients resulting from nongenetic, mainly vascular or infective, prenatal causes (Guerrini and Dobyns, 2014; Mirzaa et al., 2015; Park et al., 2020). The proportion of patients with polymicrogyria related to $A T P 1 A 2 / A 3$ remains unknown since available NGS studies on MCDs have been either performed with gene panels which included neither of the two genes (Oegema et al., 2020) or carrying out WES in small series in which no pathogenic variants in either gene emerged (Mirzaa et al., 2015; Wiszniewski et al., 2018). This study widens the clinical spectrum of ATP1A2/A3-opathies to include profound epilepsy phenotypes, with and without associated polymicrogyria. Inclusion of $A T P 1 A 2 / A 3$, in virtual panels used to interrogate WES/WGS data and in targeted-gene diagnostic panels for polymicrogyria and DEE will clarify the magnitude of their role in large series with DEEs and MCDswith these disorders.

\section{Contributors}

$\mathrm{RG}, \mathrm{BV}$ and WBD conceived the study and coordinated the experimental design of the research. AV, SB, EP, ZP, RSM, CB, GL, KLH, EEP, DM, EB, AVH, SS, VL, GiCi, CJC, GrCo, MS, $\mathrm{KC}, \mathrm{GM}, \mathrm{JCS}$, IES, WBD, and RG participated in genetic or clinical data acquisition and in the interpretation of results relevant to their domain of interest. HNN, RH and BV performed and interpreted functional experiments and structural analyses. RFH and AS acquired and interpreted neuropathological data. AV, RFH, BV, WBD, and RG drafted the manuscript and coordinated its finalisation. All authors critically reviewed the manuscript and approved the final draft. 


\section{Acknowledgements}

We thank Nina Juste and Randi Scheel, Aarhus University, for expert technical assistance. We also thank Mrs Diana Bazan for her skilful technical assistance (Neuropathology). This work was generated within the European Reference Networks EpiCARE and ITHACA.

\section{Funding}

This work was supported by grants to B.V. from the Lundbeck Foundation (grant R223-2016595) and the Danish Medical Research Council (grant 7016-00193B), grants to R.G. from the European Union Seventh Framework Programme FP7/2013 under the project DESIRE (grant 602531) and the Italian Ministry of Health and Tuscany Region (grant RF-2013-02355240) and grants to I.E.S. from the National Health and Medical Research Council of Australia (grants APP1091593, APP1104831).

Competing interests: The authors declare no competing interests.

\section{Appendix 1}

ATP1A2/A3-collaborators. Full details are provided in the Supplementary material.

Damien Sanlaville, Rani Sachdev, Ian Andrews, Francesco Mari, Anna Cavalli, Carmen Barba, Beatrice De Maria, Giampaolo Garani, Johannes R. Lemke, Mario Mastrangelo, Emily Tam, Elizabeth Donner, Helen Branson, Fabiola P. Monteiro, Fernando Kok, Katherine B Howell, Stephanie Leech, Heather Mefford, Alison Muir. 


\section{References:}

Arystarkhova E, Haq IU, Luebbert T, Mochel F, Saunders-Pullman R, Bressman SB, et al. Factors in the disease severity of ATP1A3 mutations: Impairment, misfolding, and allele competition. Neurobiol Dis 2019; 132: 104577.

Balestrini S, Mikati MA, Garcia-Roves RA, Carboni M, Hunanyan AS, Kherallah B, et al. Cardiac phenotype in ATP1A3 -related syndromes: A multicentre cohort study . Neurology 2020

Bassi MT, Bresolin N, Tonelli A, Nazos K, Crippa F, Baschirotto C, et al. A novel mutation in the ATP1A2 genes causes alternating hemiplegia of childhood. J Med Genet 2004

Blanco G. Na,K-ATPase subunit heterogeneity as a mechanism for tissue-specific ion regulation. Semin Nephrol 2005; 25: 292-303.

Blicher JU, Tietze A, Donahue MJ, Smith SA, Østergaard L. Perfusion and pH MRI in familial hemiplegic migraine with prolonged aura. Cephalalgia 2016; 36: 279-83.

Burgess R, Wang S, McTague A, Boysen KE, Yang X, Zeng Q, et al. The Genetic Landscape of Epilepsy of Infancy with Migrating Focal Seizures. Ann Neurol 2019; 86: 821-31.

De Carvalho Aguiar P, Sweadner KJ, Penniston JT, Zaremba J, Liu L, Caton M, et al. Mutations in the $\mathrm{Na}+\mathrm{K}+$-ATPase $\alpha 3$ gene ATP1A3 are associated with rapid-onset dystonia parkinsonism. Neuron 2004; 43: 169-75.

Chatron N, Cabet S, Alix E, Buenerd A, Cox P, Guibaud L, et al. A novel lethal recognizable polymicrogyric syndrome caused by ATP1A2 homozygous truncating variants. Brain 2019; 142: $3367-74$.

Dard R, Mignot C, Durr A, Lesca G, Sanlaville D, Roze E, et al. Relapsing encephalopathy with cerebellar ataxia related to an ATP1A3 mutation. Dev Med Child Neurol 2015; 57: 11836.

Deprez L, Weckhuysen S, Peeters K, Deconinck T, Claeys KG, Claes LRF, et al. Epilepsy as part of the phenotype associated with ATP1A2 mutations. Epilepsia 2008; 49: 500-8.

Friedrich T, Tavraz NN, Junghans C. ATP1A2 mutations in migraine: Seeing through the facets 
of an ion pump onto the neurobiology of disease. Front Physiol 2016; 7: 1-21.

Fry AE, Fawcett KA, Zelnik N, Yuan H, Thompson BAN, Shemer-Meiri L, et al. De novo mutations in GRIN1 cause extensive bilateral polymicrogyria. Brain 2018; 141: 698-712.

De Fusco M, Marconi R, Silvestri L, Atorino L, Rampoldi L, Morgante L, et al. Haploinsufficiency of ATP1A2 encoding the $\mathrm{Na}+/ \mathrm{K}+$ pump $\alpha 2$ subunit associated with familial hemiplegic migraine type 2. Nat Genet 2003; 33: 192-6.

Ghusayni R, Richardson JP, Uchitel J, Abdelnour E, McLean M, Prange L, et al. Magnetic resonance imaging volumetric analysis in patients with Alternating hemiplegia of childhood: A pilot study. Eur J Paediatr Neurol 2020; 26

Gressens P, Baes M, Leroux P, Lombet A, Van Veldhoven P, Janssen A, et al. Neuronal migration disorder in Zellweger mice is secondary to glutamate receptor dysfunction. Ann Neurol 2000; 48: 336-43.

Guerrini R, Dobyns WB. Malformations of cortical development: Clinical features and genetic causes. Lancet Neurol 2014; 13: 710-26.

Heinzen EL, Swoboda KJ, Hitomi Y, Gurrieri F, De Vries B, Tiziano FD, et al. De novo mutations in ATP1A3 cause alternating hemiplegia of childhood. Nat Genet 2012; 44: 1030-4.

Herrera VLM, Cova T, Sassoon D, Ruiz-Opazo N. Developmental cell-specific regulation of $\mathrm{Na}+-\mathrm{K}+-\mathrm{ATPase} \alpha 1-, \alpha 2-$, and $\alpha 3$-isoform gene expression. Am J Physiol - Cell Physiol 1994; 266: $35-5$.

Holm R, Toustrup-Jensen MS, Einholm AP, Schack VR, Andersen JP, Vilsen B. Neurological disease mutations of $\alpha 3 \mathrm{Na}+, \mathrm{K}+$-ATPase: Structural and functional perspectives and rescue of compromised function. Biochim Biophys Acta - Bioenerg 2016; 1857: 1807-28.

Ikeda K, Onaka T, Yamakado M, Nakai J, Ishikawa TO, Taketo MM, et al. Degeneration of the amygdala/piriform cortex and enhanced fear/anxiety behaviors in sodium pump $\alpha 2$ subunit (Atpla2)-deficient mice. J Neurosci 2003; 23: 4667-76.

Ikeda K, Onimaru H, Kawakami K. Knockout of sodium pump $\alpha 3$ subunit gene (Atp1a3-/-) results in perinatal seizure and defective respiratory rhythm generation. Brain Res 2017; 1666: 27-37. 
Kaplan JH. Biochemistry of Na,K-ATPase. Annu Rev Biochem 2002; 71: 511-35.

Li M, Jazayeri D, Corry B, McSweeney KM, Heinzen EL, Goldstein DB, et al. A functional correlate of severity in alternating hemiplegia of childhood. Neurobiol Dis 2015; 77: 88-93.

Liu J, Tong L, Song S, Niu Y, Li J, Wu X, et al. Novel and de novo mutations in pediatric refractory epilepsy. Mol Brain 2018; 11: 1-18.

Marzin P, Mignot C, Dorison N, Dufour L, Ville D, Kaminska A, et al. Early-onset encephalopathy with paroxysmal movement disorders and epileptic seizures without hemiplegic attacks: About three children with novel ATP1A3 mutations. Brain Dev 2018; 40: $768-74$.

McGrail KM, Phillips JM, Sweadner KJ. Immunofluorescent localization of three Na,KATPase isozymes in the rat central nervous system: Both neurons and glia can express more than one Na,K-ATPase. J Neurosci 1991; 11: 381-91.

McRae JF, Clayton S, Fitzgerald TW, Kaplanis J, Prigmore E, Rajan D, et al. Prevalence and architecture of de novo mutations in developmental disorders. Nature 2017; 542: 433-8.

Mirzaa GM, Conti V, Timms AE, Smyser CD, Ahmed S, Carter M, et al. Characterisation of mutations of the phosphoinositide-3-kinase regulatory subunit, PIK3R2, in perisylvian polymicrogyria: A next-generation sequencing study. Lancet Neurol 2015; 14: 1182-95.

Monteiro FP, Curry CJ, Hevner R, Elliott S, Fisher JH, Turocy J, et al. Biallelic loss of function variants in ATP1A2 cause hydrops fetalis, microcephaly, arthrogryposis and extensive cortical malformations. Eur J Med Genet 2020; 63: 103624.

Moseley AE, Lieske SP, Wetzel RK, James PF, He S, Shelly DA, et al. The Na,K-ATPase $\alpha 2$ isoform is expressed in neurons, and its absence disrupts neuronal activity in newborn mice. $\mathrm{J}$ Biol Chem 2003; 278: 5317-24.

Nielsen HN, Spontarelli K, Holm R, Andersen JP, Einholm AP, Artigas P, et al. Distinct effects of Q925 mutation on intracellular and extracellular $\mathrm{Na}+$ and $\mathrm{K}+$ binding to the $\mathrm{Na}+, \mathrm{K}+-$ ATPase. Sci Rep 2019; 9

Oegema R, Barakat TS, Wilke M, Stouffs K, Amrom D, Aronica E, et al. International consensus recommendations on the diagnostic work-up for malformations of cortical 
development. Nat Rev Neurol 2020

Paciorkowski AR, McDaniel SS, Jansen LA, Tully H, Tuttle E, Ghoneim DH, et al. Novel mutations in ATP1A3 associated with catastrophic early life epilepsy, episodic prolonged apnea, and postnatal microcephaly. Epilepsia 2015; 56: 422-30.

Panagiotakaki E, de Grandis E, Stagnaro M, Heinzen EL, Fons C, Sisodiya S, et al. Clinical profile of patients with ATP1A3 mutations in Alternating Hemiplegia of Childhood - A study of 155 patients. Orphanet J Rare Dis 2015; 10

Park K, Chapman T, Aldinger K, Mirzaa G, Zeiger J, Beck A, et al. The spectrum of brain malformations and disruptions in twins. Am J Med Genet Part A 2020; In press, Article ID: AJMGA61972; Article DOI: 10.1002/ajmg.a.61972

Parrini E, Marini C, Mei D, Galuppi A, Cellini E, Pucatti D, et al. Diagnostic Targeted Resequencing in 349 Patients with Drug-Resistant Pediatric Epilepsies Identifies Causative Mutations in 30 Different Genes. Hum Mutat 2017; 38: 216-25.

Pelzer N, Blom DE, Stam AH, Vijfhuizen LS, Hageman ATM, Van Vliet JA, et al. Recurrent coma and fever in familial hemiplegic migraine type 2. A prospective 15-year follow-up of a large family with a novel ATP1A2 mutation. Cephalalgia 2017; 37: 737-55.

Platzer K, Yuan H, Schütz H, Winschel A, Chen W, Hu C, et al. GRIN2B encephalopathy: Novel findings on phenotype, variant clustering, functional consequences and treatment aspects. J Med Genet 2017; 54: 460-70.

Prontera P, Sarchielli P, Caproni S, Bedetti C, Cupini LM, Calabresi P, et al. Epilepsy in hemiplegic migraine: Genetic mutations and clinical implications. Cephalalgia 2018; 38: 36173.

Reinhard L, Tidow H, Clausen MJ, Nissen P. Na+,K+-ATPase as a docking station: Proteinprotein complexes of the $\mathrm{Na}+, \mathrm{K}+-\mathrm{ATPase}$. Cell Mol Life Sci 2013; 70: 205-22.

Retterer K, Juusola J, Cho MT, Vitazka P, Millan F, Gibellini F, et al. Clinical application of whole-exome sequencing across clinical indications. Genet Med 2016; 18: 696-704.

Rosewich H, Ohlenbusch A, Huppke P, Schlotawa L, Baethmann M, Carrilho I, et al. The expanding clinical and genetic spectrum of ATP1A3-related disorders. Neurology 2014; 82: 
$945-55$.

Rosewich H, Sweney MT, Debrosse S, Ess K, Ozelius L, Andermann E, et al. Research conference summary from the 2014 International Task Force on ATP1A3-related disorders. Neurol Genet 2017; 3

Rosewich H, Thiele H, Ohlenbusch A, Maschke U, Altmüller J, Frommolt P, et al. Heterozygous de-novo mutations in ATP1A3 in patients with alternating hemiplegia of childhood: A whole-exome sequencing gene-identification study. Lancet Neurol 2012; 11: 764-73.

Roth C, Freilinger T, Kirovski G, Dunkel J, Shah Y, Wilken B, et al. Clinical spectrum in three families with familial hemiplegic migraine type 2 including a novel mutation in the ATP1A2 gene. Cephalalgia 2014; 34: 183-90.

Roubergue A, Roze E, Vuillaumier-Barrot S, Fontenille MJ, Méneret A, Vidailhet M, et al. The multiple faces of the ATP1A3-related dystonic movement disorder. Mov Disord 2013; 28

Sasaki M, Ishii A, Saito Y, Hirose S. Progressive Brain Atrophy in Alternating Hemiplegia of Childhood. Mov Disord Clin Pract 2017; 4: 406-11.

Scheffer IE, Berkovic S, Capovilla G, Connolly MB, French J, Guilhoto L, et al. ILAE classification of the epilepsies: Position paper of the ILAE Commission for Classification and Terminology. Epilepsia 2017; 58: 512-21.

Severino M, Pisciotta L, Tortora D, Toselli B, Stagnaro M, Cordani R, et al. White matter and cerebellar involvement in alternating hemiplegia of childhood. J Neurol 2020; 267

Smith RS, Kenny CJ, Ganesh V, Jang A, Borges-Monroy R, Partlow JN, et al. Sodium Channel SCN3A (NaV1.3) Regulation of Human Cerebral Cortical Folding and Oral Motor Development. Neuron 2018; 99: 905-913.e7.

Smith RS, Walsh CA. Ion Channel Functions in Early Brain Development. Trends Neurosci 2020; 43: 103-14.

Sweadner KJ, Arystarkhova E, Penniston JT, Swoboda KJ, Brashear A, Ozelius LJ. Genotypestructure-phenotype relationships diverge in paralogs ATP1A1, ATP1A2, and ATP1A3. Neurol Genet 2019; 5 
Swoboda KJ, Kanavakis E, Xaidara A, Johnson JE, Leppert MF, Schlesinger-Massart MB, et al. Alternating hemiplegia of childhood or familial hemiplegic migraine?: A novel ATP1A2 mutation. Ann Neurol 2004; 55: 884-7.

Tavraz NN, Dürr KL, Koenderink JB, Freilinger T, Bamberg E, Dichgans M, et al. Impaired plasma membrane targeting or protein stability by certain ATP1A2 mutations identified in sporadic or familial hemiplegic migraine. Channels 2009; 3: 82-7.

Ueda K, Serajee F, Huq AM. Clinical benefit of NMDA receptor antagonists in a patient with ATP1A2 gene mutation. Pediatrics 2018; 141: S390-4.

De Vries B, Freilinger T, Vanmolkot KRJ, Koenderink JB, Stam AH, Terwindt GM, et al. Systematic analysis of three FHM genes in 39 sporadic patients with hemiplegic migraine. Neurology 2007; 69: 2170-6.

Weigand KM, Messchaert M, Swarts HGP, Russel FGM, Koenderink JB. Alternating Hemiplegia of Childhood mutations have a differential effect on $\mathrm{Na}+, \mathrm{K}+-\mathrm{ATP}$ ase activity and ouabain binding. Biochim Biophys Acta - Mol Basis Dis 2014; 1842: 1010-6.

Wiszniewski W, Gawlinski P, Gambin T, Bekiesinska-Figatowska M, Obersztyn E, AntczakMarach D, et al. Comprehensive genomic analysis of patients with disorders of cerebral cortical development. Eur J Hum Genet 2018; 26

Yang X, Yang X, Chen J, Li S, Zeng Q, Huang AY, et al. ATP1A3 mosaicism in families with alternating hemiplegia of childhood. Clin Genet 2019; 96: 43-52.

Zaman T, Helbig KL, Clatot J, Thompson CH, Kang SK, Stouffs K, et al. SCN3A-Related Neurodevelopmental Disorder: A Spectrum of Epilepsy and Brain Malformation. Ann Neurol 2020; 88: 348-62. 


\section{Legend to figures}

Figure 1 - Brain MRI in 9 patients with polymicrogyria. From top to bottom, representative brain MRI findings in Patients 6, 7, 11-17 and 20 with an indication of the mutations and age at which imaging was performed. For each patient four cuts are presented, which include axial (column A), coronal (column B), sagittal through the sylvian fissure (column C), and sagittal through the midline (column D) images. Sequences are at 1.5 to $3 \mathrm{~T}$ and include T1-weighted, T2 weighted and fluid-attenuated inversion recovery (FLAIR) images. All patients have polymicrogyria with abnormal cortical infoldings and packed microgyri, combined with abnormal sulcation. A recurrent feature, present in all patients, is an abnormal sylvian fissure with thickened cortex, pronounced infolding and vertically oriented axis. These abnormalities are better visible in the A column (enlarged sylvian space), B column (infolding) and C column (cortical thickening and verticalized sylvian fissure). There are some differences in individual patients. In Patient 6, polymicrogyria is diffuse and more prominent posteriorly. In Patient 7, the cortical abnormality is asymmetrical, due to a smaller right hemisphere. In Patient 11 too, there is asymmetry with the right hemisphere being more severely affected and smaller. In Patients 13,14, 16 and 17, polymicrogyria is almost exclusively bilateral perisylvian, sparing the remaining cortex. In Patient 15 too, who is the mother of Patient 14, the cortical abnormality is perisylvian but unilateral only involving the right hemisphere. In Patient 17, there is diffuse polymicrogyria with more severe perisylvian involvement. In line D, midline cuts show thickening of the corpus callosum in Patients 6,14 and 15. There is some degree of vermian hypoplasia in Patients 7 and 17.

Figure 2 - Neuropathology of patient 11, age 4 months (A3-K764del). (A-C) Macroscopic views of the brain from posterior (A), right (B), and left (C) sides demonstrated overall small cerebral hemispheres, with externally apparent pachygyria (asterisks) involving pericentral cortex bilaterally. (D, E) Coronal slices through anterior (D) and middle (E) regions of the hemispheres showed thick cortex corresponding to externally apparent pachygyria (asterisks), and thin but intact genu of the corpus callosum (white arrow, D). (F, G) Histology of the brain slices demonstrated leptomeningeal glioneuronal heterotopia (black arrowheads) covering cortex that appeared externally pachygyric, but histologically showed features of 4-layered polymicrogyria, also seen in other cortical areas (black arrows). Callosal fibers did not cross the midline posteriorly, but instead formed Probst bundles (PB). 


\section{Figure 3 - Neuropathology of patient 23, age 1 day (A2-R279Gfs4*, homozygous).} Macroscopic brain abnormalities (A-F). (A) Left brain, lateral view. Blood vessels were congested; the leptomeninges contained subarachnoid haemorrhage (agonal) and were adherent to the brain surface. (B) Right hemisphere, medial view. The corpus callosum was thin (white arrow). (C) Brainstem and right cerebellum, medial view. The cerebellum was moderately hypoplastic. (D, E) Coronal slices of brain, level of amygdala (D) and anterior hippocampus (E). The basal ganglia and thalamus were moderately hypoplastic; the cortex was diffusely polymicrogyric, except for the hippocampi (black arrows), which appeared relatively wellformed. (F) The occipital lobes (coronal slices) exhibited polymicrogyria and moderate colpocephaly. Histopathology (H\&E; G-N) (G) Frontal cortex, coronal section (midline right). The cortex was diffusely polymicrogyric, had an attached external rind of leptomeningeal glioneuronal heterotopia, and contained focal calcifications (arrows). The corpus callosum (CC) was small, but crossed the midline. (H) Medial temporal lobe, coronal section. The hippocampus was well-formed, including the dentate gyrus (DG), while neocortex was polymicrogyric. Focal calcifications (arrows) were noted in deep white matter. (I) Leptomeningeal glioneuronal heterotopia (Het) covered much of the cortical surface. Note focal parenchymal calcifications (arrows). (J) Leptomeninges with focal calcifications (arrows). (K) Cortical blood vessel with mineralized walls (arrows). (L) Meningeal blood vessel with medial calcifications (arrows), and intimal hyperplasia possibly due to thrombosis and recanalization. Scale bars: (A) $1 \mathrm{~cm}$ for (A-F); (G), $1 \mathrm{~mm}$ for (G,H); (I), $100 \mu \mathrm{m}$ for (I,J); $50 \mu \mathrm{m}$ for (K,L).

\section{Figure 4: Overview of the structural location of ATP1A2 and ATP1A3 disease mutations}

\section{in the cohort}

The NKA $\alpha$-subunit consists of three major cytoplasmic domains A ("actuator"), N ("nucleotide-binding"), and P ("phosphorylation") linked to a membrane domain composed of ten transmembrane helices (M1-M10) with their intervening loops on both membrane sides. The $\beta$-subunit (PDB ID 3WGV, chain B, teal color) consists of a transmembrane helix $\beta \mathrm{m}$ and an extracellular domain that interacts with the $\alpha$-subunit loop connecting M7 and M8 (L7-8 loop). Amino acid residues targeted by the mutations in the cohort are shown in blue (ATP1A2 mutations) and red (ATP1A3 mutations) in the structure of the ATP1A1 $\alpha$-subunit in E1 form (PDB ID 3WGV, chain A, grey color) with $3 \mathrm{Na}^{+}$(golden spheres labelled I, II, and III according to common nomenclature) and $\mathrm{ADP}$ with phosphate analog $\mathrm{AlF}_{4}$ and the phosphorylated aspartate in green. 


\section{Figure 5: Relative phosphorylation/expression and turnover rate for ATPase activity with} NKA reaction cycle

(A) Phosphorylation level of stably expressed ATP1A2 and ATP1A3 wild type and mutants. The phosphorylation level ( $\mathrm{pmol} / \mathrm{mg}$ total plasma membrane protein) is shown in \% of wild type. Phosphorylation was determined under stoichiometric conditions, thus reflecting the expression level (“active site concentration"). (B) Turnover rate $\left(\mathrm{min}^{-1}\right)$ determined by relating the maximum NKA activity to the phosphorylation level. (C) Phosphorylation level of transiently expressed ATP1A2 and ATP1A3 wild type and mutants determined as described for (A). The NKA activity per mg total plasma membrane protein, calculated by multiplying the turnover rate by the phosphorylation level, was 17\% (A2-I293M), 36\% (A2-R593Q), 22\% (A2R908Q), 30\% (A3-P972del), and 33\% (A3-D887Y), relative to wild-type, indicating that the function of these mutants was substantially impaired despite their ability to support cell growth. (D) Reaction cycle of NKA. E1 and E1P are $\mathrm{Na}^{+}$selective conformations, whereas E2 and E2P are $\mathrm{K}^{+}$selective conformations. Phosphorylation by ATP (indicated by “P”). The cytoplasmic and extracellular sides are indicated by c and e, respectively, see detailed description in supplementary legend to figure 5 (Supplementary Materials) (Holm et al., 2016).

\section{Figure 6: $\mathrm{Na}^{+}$and $\mathrm{K}^{+}$affinity of ATP1A2/A3 mutants}

(A) $\mathrm{Na}^{+}$affinity determined from $\mathrm{Na}^{+}$activation of phosphorylation. The binding of three $\mathrm{Na}^{+}$ to the E1 form activates phosphorylation from ATP. Phosphorylation was measured at the indicated concentrations of $\mathrm{Na}^{+}$. Symbols with error bars represent mean $\pm \mathrm{SD}$. Each line represents the best fit of a Hill equation. The apparent $\mathrm{Na}^{+}$affinities $\left(\mathrm{K}_{0.5}\right.$ values $)$ and Hill coefficients $\left(\mathrm{n}_{\mathrm{H}}\right)$ were as follows. Stable expression (upper panels): A2-wt (wild-type), $534 \pm$ $52 \mu \mathrm{M}\left(\mathrm{n}_{\mathrm{H}}=2.0\right) ;$ A2-I293M, $2790 \pm 592 \mu \mathrm{M}\left(\mathrm{n}_{\mathrm{H}}=1.7\right)$; A2-R593Q, $384 \pm 24 \mu \mathrm{M}\left(\mathrm{n}_{\mathrm{H}}=2.6\right)$; A2-R908Q, $601 \pm 81 \mu \mathrm{M}\left(\mathrm{n}_{\mathrm{H}}=1.7\right)$; A3-wt, $916 \pm 105 \mu \mathrm{M}\left(\mathrm{n}_{\mathrm{H}}=2.4\right)$; A3-D887Y, $1260 \pm 93$ $\mu \mathrm{M}\left(\mathrm{n}_{\mathrm{H}}=2.1\right)$; A3-P972del, $1331 \pm 303 \mu \mathrm{M}\left(\mathrm{n}_{\mathrm{H}}=1.2\right)$. Transient expression (lower panels): A2-wt, $595 \pm 80 \mu \mathrm{M}\left(\mathrm{n}_{\mathrm{H}}=2.5\right)$; A2-G366A, 3079 $\pm 192 \mu \mathrm{M}\left(\mathrm{n}_{\mathrm{H}}=1.3\right)$; A3-wt, $1120 \pm 127 \mu \mathrm{M}$ $\left(\mathrm{n}_{\mathrm{H}}=2.1\right)$; A3-L292R, $3013 \pm 254 \mu \mathrm{M}\left(\mathrm{n}_{\mathrm{H}}=1.9\right)$; A3-G316V, $272 \pm 47 \mu \mathrm{M}\left(\mathrm{n}_{\mathrm{H}}=1.3\right)$. The apparent $\mathrm{Na}^{+}$affinity was reduced 3- to 5-fold ( $\mathrm{K}_{0.5}$ increased) for A2-I293M, A2-G366A, and A3-L292R, and increased 4-fold for A3-G316V ( $\mathrm{K}_{0.5}$ decreased). For A3-P972del, the cooperativity of $\mathrm{Na}^{+}$binding at the three sites was severely reduced (Hill coefficient 1.2 vs. 2.4 for the wild-type). (B) $\mathrm{K}^{+}$affinity determined from $\mathrm{K}^{+}$inhibition of phosphorylation. Accumulation of phosphoenzyme is inhibited by $\mathrm{K}^{+}$, because $\mathrm{K}^{+}$activates dephosphorylation of E2P and competes with $\mathrm{Na}^{+}$for binding to E1 (cf. reaction cycle in Fig. 5). Phosphorylation 
was measured at the indicated concentrations of $\mathrm{K}^{+}$on transiently expressed NKA enzyme. Symbols with error bars represent mean \pm SD. Each line represents the best fit of the Hill equation for inhibitory ligand binding (Nielsen et al., 2019). The apparent $\mathrm{K}^{+}$affinities $\left(\mathrm{K}_{0.5}\right.$ values) were as follows: A2-wt, $188 \pm 43 \mu \mathrm{M}$; A2-G366A, >100 mM; A3-wt, $281 \pm 63 \mu \mathrm{M}$; A3-L292R, $4570 \pm 700 \mu \mathrm{M}$; A3-G316V, >10 mM. Hence, A3-L292R and A3-G316V showed 16- and $>35$-fold reduction of apparent $\mathrm{K}^{+}$affinity, and A2-G366A was totally insensitive to $\mathrm{K}^{+}$.

\section{Figure 7 - Distribution of ATP1A2/A3 mutations across their associated phenotypes.}

The pie charts show the distribution of heterozygous mutations of ATP1A2 (left) and ATP1A3 (right) across their associated phenotypes. We identified 92 ATP1A2 and 136 ATP1A3 heterozygous mutations in the Human Gene Mutation Database (https://portal.biobaseinternational.com), and stratified them based on the reported phenotypes, adding to previously published data the 11 novel mutations ( 2 in ATP1A2 and 9 in ATP1A3) we reported in this series. Collectively, up to $5 \%$ of ATP1A2 (6/94) and 12\% of ATP1A3 (18/145) mutations can be associated with developmental and epileptic encephalopathy. Polymicrogyria can be associated with $1 \%$ of $A T P 1 A 2$ and $5.5 \%$ of $A T P 1 A 3$ mutations (yellow-dashed area). Abbreviations: AHC, alternating hemiplegia of childhood; DYT, dystonia; DEE, developmental and epileptic encephalopathy (this term includes epileptic encephalopathies and developmental encephalopathies with epilepsy); FHM, familial hemiplegic migraine; PMG, polymicrogyria; RDP, rapid-onset dystonia-parkinsonism; RP, rare phenotypes (this sub-group includes CAPOS, RECA, ataxia and non-specified nervous system abnormalities). 
Table 1

\begin{tabular}{|c|c|c|c|c|c|c|c|c|c|}
\hline & \multicolumn{6}{|c|}{$A T P 1 A 2$, heterozygous } & \multicolumn{3}{|c|}{$A T P 1 A 3$, heterozygous } \\
\hline $\begin{array}{l}\text { Patient } \\
\text { Nr/sex }\end{array}$ & $1 / \mathbf{M}$ & $2 / \mathbf{M}$ & $3 / \mathbf{M}$ & $4 / F$ & 5/M & 6/M & $7 / \mathrm{F}$ & $8 / F$ & $9 / F$ \\
\hline $\begin{array}{c}\text { Mutation } \\
\text { (protein)/ } \\
\text { abbreviati } \\
\text { on }\end{array}$ & $\begin{array}{c}\mathrm{p} .(\text { Ile29 } \\
\text { 3Met)/A } \\
2- \\
\text { I293M }\end{array}$ & $\begin{array}{c}\text { p.(Cys34 } \\
1 \mathrm{Phe}) / \mathrm{A} \\
2-\mathrm{C} 341 \mathrm{~F}\end{array}$ & $\begin{array}{c}\text { p.(Gly36 } \\
\text { 6Ala)**/ } \\
\text { A2- } \\
\text { G366A }\end{array}$ & $\begin{array}{c}\text { p.(Gly36 } \\
\text { 6Ala)**/ } \\
\text { A2- } \\
\text { G366A }\end{array}$ & $\begin{array}{c}\text { p. }(\text { Arg59 } \\
\text { 3Gln)/A } \\
2- \\
\text { R593Q }\end{array}$ & $\begin{array}{c}\text { p.(Arg90 } \\
8 \mathrm{Gln}) / \mathrm{A} \\
2- \\
\text { R908Q }\end{array}$ & $\begin{array}{l}\text { p.(Leu29 } \\
\text { 2Arg)/A } \\
\text { 3-L292R }\end{array}$ & $\begin{array}{c}\text { p.(Gly31 } \\
\text { 6Val)/A } \\
\text { 3- } \\
\text { G316V }\end{array}$ & $\begin{array}{c}\text { p.(Ser361 } \\
\text { Pro)**/A } \\
3-S 361 P\end{array}$ \\
\hline $\begin{array}{c}\text { Inherited/ } \\
\text { De } \\
\text { novo/type } \\
\text { of } \\
\text { analysis } \\
\end{array}$ & $\begin{array}{c}\text { De } \\
\text { novo/W } \\
\text { ES }\end{array}$ & $\begin{array}{c}\text { De } \\
\text { novo/TG } \\
\text { P }\end{array}$ & $\begin{array}{c}\text { De } \\
\text { novo/WE } \\
\mathrm{S}\end{array}$ & $\begin{array}{c}\text { De } \\
\text { novo/WE } \\
\text { S }\end{array}$ & $\begin{array}{c}\text { De } \\
\text { novo/TG } \\
\text { P }\end{array}$ & $\begin{array}{c}\text { De } \\
\text { novo/W } \\
\text { ES }\end{array}$ & $\begin{array}{c}\text { De } \\
\text { novo/W } \\
\text { GS }\end{array}$ & $\begin{array}{c}\text { De } \\
\text { novo/W } \\
\text { ES }\end{array}$ & $\begin{array}{c}\text { De } \\
\text { novo/WE } \\
\mathrm{S}\end{array}$ \\
\hline 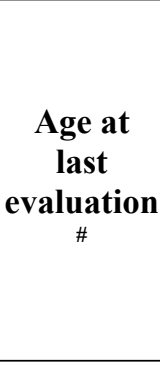 & $12 y$ & $5 y$ & $\begin{array}{c}1 \mathrm{y}, \\
\text { deceased } \\
\text { (respirato } \\
\text { ry } \\
\text { complicat } \\
\text { ions of } \\
\text { status } \\
\text { epilepticu } \\
\text { s) }\end{array}$ & $\begin{array}{l}1 \mathrm{~m} 3 \mathrm{w}, \\
\text { deceased } \\
\text { (respirato } \\
\text { ry } \\
\text { complicat } \\
\text { ions of } \\
\text { status } \\
\text { epilepticu } \\
\text { s) }\end{array}$ & $8 y$ & $11 y$ & $\begin{array}{c}2 \mathrm{~m}, \\
\text { deceased } \\
\text { (respirat } \\
\text { ory } \\
\text { complica } \\
\text { tions of } \\
\text { status } \\
\text { epileptic } \\
\text { us) }\end{array}$ & $6 y$ & $7 y$ \\
\hline $\begin{array}{c}\text { Prenatal/ } \\
\text { perinatal } \\
\text { findings }\end{array}$ & $\begin{array}{c}\text { Unrema } \\
\text { rkable }\end{array}$ & $\begin{array}{c}\text { Unremar } \\
\text { kable }\end{array}$ & $\begin{array}{c}\text { Dizygotic } \\
\text { twin } \\
\text { (twin } \\
\text { unaffecte } \\
\text { d) }\end{array}$ & $\begin{array}{l}\text { Caesarea } \\
\mathrm{n} \text { section } \\
\text { at } 38 \mathrm{w} \\
\text { because } \\
\text { of } \\
\text { oligohydr } \\
\text { amnios }\end{array}$ & $\begin{array}{c}\text { Unremar } \\
\text { kable }\end{array}$ & $\begin{array}{c}\text { Unremar } \\
\text { kable }\end{array}$ & $\begin{array}{c}\text { Antenata } \\
1 \\
\text { 'hiccoug } \\
\text { h'. Apgar } \\
\text { 3/6/6 }\end{array}$ & $\begin{array}{c}\text { Unremar } \\
\text { kable }\end{array}$ & $\begin{array}{c}\text { Unremar } \\
\text { kable }\end{array}$ \\
\hline $\begin{array}{c}\text { Age at } \\
\text { seizure } \\
\text { onset/ } \\
\text { type/sever } \\
\text { ity }\end{array}$ & $\begin{array}{c}18 \mathrm{~m} / \text { foc } \\
\text { al } \\
\text { dyscogn } \\
\text { itive, } \\
\text { left } \\
\text { unilater } \\
\text { al } \\
\text { clonic/i } \\
\text { nitially } \\
\text { frequent } \\
\text {; seizure } \\
\text { free } \\
\text { since } \\
\text { age } 5 y\end{array}$ & $\begin{array}{l}\text { 3w/tonic } \\
\text { spasms, } \\
\text { migratin } \\
\text { g focal } \\
\text { seizures/ } \\
\text { severe }\end{array}$ & $\begin{array}{l}1 \mathrm{~m} / \text { prolo } \\
\text { nged } \\
\text { migrating } \\
\text { focal } \\
\text { seizures } \\
\text { with } \\
\text { apnea, } \\
\text { cyanosis } \\
\text { and } \\
\text { hypertonu } \\
\text { s } \\
\text { evolving } \\
\text { to super- } \\
\text { refractory } \\
\text { status } \\
\text { epilepticu } \\
\text { s }\end{array}$ & $\begin{array}{l}\text { 10d/prolo } \\
\text { nged } \\
\text { migrating } \\
\text { focal } \\
\text { seizures } \\
\text { evolving } \\
\text { to super- } \\
\text { refractory } \\
\text { status } \\
\text { epilepticu } \\
\text { s }\end{array}$ & $\begin{array}{l}\text { 8y/gener } \\
\text { alised } \\
\text { TC with } \\
\text { prolonge } \\
\text { d post- } \\
\text { ictal } \\
\text { confusio } \\
\text { n/recent } \\
\text { onset }\end{array}$ & $\begin{array}{c}8 \mathrm{y} / \text { focal } \\
\text { onset } \\
\text { with } \\
\text { bilateral } \\
\text { posturin } \\
\text { g/rare }\end{array}$ & \begin{tabular}{|} 
At \\
birth/mi \\
grating \\
focal \\
seizures \\
with \\
apnea/ \\
almost \\
continuo \\
us \\
seizures, \\
status \\
epileptic \\
us/sever \\
e
\end{tabular} & $\begin{array}{c}4 \mathrm{y} / \\
\text { migratin } \\
\mathrm{g} \text { focal } \\
\text { and } \\
\text { generalis } \\
\text { ed } \\
\text { seizures/ } \\
\text { severe }\end{array}$ & $\begin{array}{l}5 \mathrm{~m} / \text { focal } \\
\text { temporal } \\
\text { status } \\
\text { epilepticu } \\
\text { s with } \\
\text { ictal } \\
\text { vomiting } \\
\text { at onset, } \\
\text { hemiclon } \\
\text { ic/severe }\end{array}$ \\
\hline $\begin{array}{c}\text { Interictal } \\
\text { EEG }\end{array}$ & $\begin{array}{c}\text { Bilateral } \\
\text { occipital } \\
\text { discharg } \\
\text { es }\end{array}$ & $\begin{array}{c}\text { Slow } \\
\text { backgro } \\
\text { und, } \\
\text { multifoc } \\
\text { al sharp } \\
\text { waves }\end{array}$ & $\begin{array}{c}\text { Burst } \\
\text { suppressi } \\
\text { on, } \\
\text { multifoca } \\
1 \\
\text { discharge } \\
\text { s }\end{array}$ & $\begin{array}{c}\text { Slow } \\
\text { backgrou } \\
\text { nd } \\
\text { activity, } \\
\text { multifoca } \\
1 \\
\text { discharge } \\
\text { s }\end{array}$ & NA & $\begin{array}{c}\text { Continu } \\
\text { ous } \\
\text { bilateral } \\
\text { fronto- } \\
\text { temporal } \\
\text { and } \\
\text { multifoc } \\
\text { al } \\
\text { discharg } \\
\text { es } \\
\end{array}$ & $\begin{array}{c}\text { Multifoc } \\
\text { al } \\
\text { discharg } \\
\text { es }\end{array}$ & $\begin{array}{c}\text { Slow } \\
\text { backgro } \\
\text { und, } \\
\text { multifoc } \\
\text { al } \\
\text { discharg } \\
\text { es }\end{array}$ & $\begin{array}{c}\text { Slow } \\
\text { backgrou } \\
\text { nd, } \\
\text { multifoca } \\
1 \\
\text { discharge } \\
\text { s }\end{array}$ \\
\hline
\end{tabular}




\begin{tabular}{|c|c|c|c|c|c|c|c|c|c|}
\hline & \multicolumn{6}{|c|}{$A T P 1 A 2$, heterozygous } & \multicolumn{3}{|c|}{$A T P 1 A 3$, heterozygous } \\
\hline $\begin{array}{l}\text { Patient } \\
\text { Nr/sex }\end{array}$ & $1 / \mathbf{M}$ & $2 / \mathbf{M}$ & 3/M & $4 / F$ & $5 / \mathbf{M}$ & 6/M & $7 / F$ & $8 / \mathbf{F}$ & $9 / \mathbf{F}$ \\
\hline $\begin{array}{c}\text { Brain } \\
\text { MRI: } \\
\text { age/findin } \\
\text { gs }\end{array}$ & $\begin{array}{c}18 \\
\mathrm{~m} / \mathrm{bilate} \\
\text { ral } \\
\text { peritrigo } \\
\text { nal high } \\
\text { signal } \\
\text { intensity }\end{array}$ & $\begin{array}{c}1 \mathrm{~m} / \text { thin } \\
\text { corpus } \\
\text { callosum } \\
\text {, frontal } \\
\text { and } \\
\text { cerebella } \\
\mathrm{r} \\
\text { atrophy; } \\
13 \mathrm{~m} / \\
\text { worseni } \\
\text { ng of } \\
\text { atrophy, } \\
\text { dilated } \\
\text { ventricle } \\
\text { s }\end{array}$ & $\begin{array}{c}1 \mathrm{~m} / \text { norm } \\
\text { al }\end{array}$ & $\begin{array}{c}1 \mathrm{~m} / \text { norm } \\
\text { al }\end{array}$ & $\begin{array}{c}\text { 8y/norm } \\
\text { al }\end{array}$ & $\begin{array}{c}\text { 3y/bilate } \\
\text { ral } \\
\text { perisylvi } \\
\text { an } \\
\text { polymicr } \\
\text { ogyria, } \\
\text { thickene } \\
\text { d corpus } \\
\text { callosum }\end{array}$ & $\begin{array}{l}\text { 4d/exten } \\
\text { sive } \\
\text { bilateral, } \\
\text { perisylvi } \\
\text { an } \\
\text { predomi } \\
\text { nant, } \\
\text { polymicr } \\
\text { ogyria }\end{array}$ & $\begin{array}{c}7 \mathrm{~m} / \text { diffu } \\
\text { se brain, } \\
\text { brainste } \\
\mathrm{m} \text { and } \\
\text { cerebella } \\
\mathrm{r} \\
\text { atrophy, } \\
\text { thin } \\
\text { corpus } \\
\text { callosu } \\
\text { m; } 3 \\
\text { y/worse } \\
\text { ning of } \\
\text { atrophy }\end{array}$ & $\begin{array}{c}8 \mathrm{~m} \text { and } \\
30 \mathrm{~m} / \mathrm{nor} \\
\mathrm{mal}\end{array}$ \\
\hline $\begin{array}{c}\text { Microcep } \\
\text { haly } \\
\text { (OFC <- } \\
\text { 2SD) } \\
\end{array}$ & No & No & No & $\begin{array}{c}\text { Yes, } \\
\text { postnatal } \\
(-2 \mathrm{SD})\end{array}$ & No & $\begin{array}{c}\text { Yes, } \\
\text { postnatal } \\
(-2.5 \mathrm{SD})\end{array}$ & $\begin{array}{c}\text { Yes, } \\
\text { postnatal } \\
(-2.4 \mathrm{SD})\end{array}$ & No & $\begin{array}{c}\text { Yes, } \\
\text { postnatal } \\
(-2.8 \mathrm{SD})\end{array}$ \\
\hline $\begin{array}{c}\text { Other } \\
\text { Neurologi } \\
\text { cal } \\
\text { Features }\end{array}$ & \begin{tabular}{|c} 
Moderat \\
$\mathrm{e}$ \\
develop \\
mental \\
delay/I \\
$\mathrm{D}$, \\
ADHD, \\
dubious \\
episodes \\
of left \\
sided \\
hemipar \\
esis \\
likely \\
postictal
\end{tabular} & $\begin{array}{c}\text { Severe } \\
\text { develop } \\
\text { mental } \\
\text { delay, } \\
\text { nonverb } \\
\text { al, } \\
\text { severe } \\
\text { axial } \\
\text { hypotoni } \\
\text { a, } \\
\text { dyskinet } \\
\text { ic } \\
\text { quadripa } \\
\text { resis }\end{array}$ & $\begin{array}{l}\text { Profound } \\
\text { delay, } \\
\text { severe } \\
\text { hypotonia }\end{array}$ & $\begin{array}{l}\text { Profound } \\
\text { delay, } \\
\text { severe } \\
\text { hypotonia } \\
\text {, poor eye } \\
\text { contact }\end{array}$ & No & $\begin{array}{c}\text { Moderat } \\
\text { e } \\
\text { develop } \\
\text { mental } \\
\text { delay, } \\
\text { hypotoni } \\
\text { c } \\
\text { quadripa }\end{array}$ & $\begin{array}{l}\text { Profoun } \\
\text { d delay, } \\
\text { severe } \\
\text { hypotoni } \\
\text { a }\end{array}$ & $\begin{array}{l}\text { Profoun } \\
\text { d delay, } \\
\text { hypotoni } \\
\text { c } \\
\text { quadripa } \\
\text { resis }\end{array}$ & $\begin{array}{c}\text { Moderate } \\
\text { developm } \\
\text { ental } \\
\text { delay }\end{array}$ \\
\hline $\begin{array}{l}\text { Additiona } \\
\text { I Clinical } \\
\text { findings } \\
\text { and ECG }\end{array}$ & $\begin{array}{c}\text { Sleep } \\
\text { apnea } \\
\text { (obstruc } \\
\text { tive?/ } \\
\text { central? } \\
\text { ), cyclic } \\
\text { vomitin } \\
\text { g; ECG } \\
\text { unavaila } \\
\text { ble }\end{array}$ & $\begin{array}{c}\text { No; } \\
\text { ECG: } \\
\text { normal }\end{array}$ & $\begin{array}{c}\text { No; ECG: } \\
\text { normal }\end{array}$ & $\begin{array}{c}\text { Down } \\
\text { syndrome } \\
\text { with } \\
\text { complex } \\
\text { congenita } \\
1 \text { heart } \\
\text { disease; } \\
\text { ECG: } \\
\text { normal }\end{array}$ & $\begin{array}{c}\text { No; } \\
\text { ECG } \\
\text { unavaila } \\
\text { ble }\end{array}$ & $\begin{array}{c}\text { No; } \\
\text { ECG: } \\
\text { normal }\end{array}$ & $\begin{array}{c}\text { Right } \\
\text { ventricul } \\
\text { ar } \\
\text { hypertro } \\
\text { phy }\end{array}$ & $\begin{array}{c}\text { No; } \\
\text { ECG: } \\
\text { normal }\end{array}$ & $\begin{array}{c}\text { No; } \\
\text { ECG: } \\
\text { normal }\end{array}$ \\
\hline $\begin{array}{l}\text { Novel/Ref } \\
\text {. (for } \\
\text { previously } \\
\text { reported } \\
\text { mutations } \\
\text { ) }\end{array}$ & $\begin{array}{c}\text { (Ueda et } \\
\text { al., } \\
\text { 2018) }\end{array}$ & Novel & $\begin{array}{l}\text { (Parrini et } \\
\text { al., 2017) }\end{array}$ & $\begin{array}{l}\text { (Parrini et } \\
\text { al., 2017) }\end{array}$ & Novel & $\begin{array}{c}(\mathrm{De} \\
\text { Vries et } \\
\text { al., } \\
2007)\end{array}$ & Novel & Novel & Novel \\
\hline
\end{tabular}


Table 1 (continues)

\begin{tabular}{|c|c|c|c|c|c|c|c|c|c|}
\hline \multirow[b]{2}{*}{$\begin{array}{l}\text { Patient } \\
\text { Nr/sex }\end{array}$} & \multicolumn{9}{|c|}{$A T P 1 A 3$, heterozygous } \\
\hline & $10 / M$ & $11 / F$ & $12 / \mathrm{M}$ & $13 / \mathbf{M}$ & 14/M & $15 / F$ & $16 / M$ & $17 / F$ & $18 / F$ \\
\hline $\begin{array}{c}\text { Mutation } \\
\text { (protein)/a } \\
\text { bbreviatio } \\
\text { n }\end{array}$ & $\begin{array}{l}\text { p.(Asp60 } \\
\text { 9Tyr)/A3 } \\
\text {-D609Y }\end{array}$ & $\begin{array}{c}\text { p.(Lys76 } \\
\text { 4del)/A3 } \\
- \\
\text { K764del } \\
\end{array}$ & $\begin{array}{l}\text { p.(Pro77 } \\
\text { 5Arg)/A } \\
\text { 3-P775R }\end{array}$ & $\begin{array}{l}\text { p. }(\text { Asp80 } \\
1 \text { Asn }) / A \\
3-D 801 N\end{array}$ & $\begin{array}{l}\text { p.(Asp88 } \\
7 \mathrm{Tyr}) / \mathrm{A} 3 \\
-\mathrm{D} 887 \mathrm{Y}\end{array}$ & $\begin{array}{l}\text { p. }(\text { Asp88 } \\
7 \mathrm{Tyr}) / \mathrm{A} 3 \\
-\mathrm{D} 887 \mathrm{Y}\end{array}$ & $\begin{array}{l}\text { p.(Leu88 } \\
\text { 8Pro)/A3 } \\
\text {-L888P }\end{array}$ & $\begin{array}{c}\text { p.(Gly89 } \\
\text { 3Trp)/A } \\
\text { 3- } \\
\text { G893W }\end{array}$ & $\begin{array}{c}\text { p.(Pro97 } \\
\text { 2del)/A3 } \\
- \\
\text { P972del }\end{array}$ \\
\hline $\begin{array}{c}\text { Inherited/ } \\
\text { De } \\
\text { novo/type } \\
\text { of analysis }\end{array}$ & $\begin{array}{c}\text { De } \\
\text { novo/WE } \\
\mathrm{S}\end{array}$ & $\begin{array}{c}\text { De } \\
\text { novo/W } \\
\text { ES }\end{array}$ & $\begin{array}{c}\text { De } \\
\text { novo/W } \\
\text { ES }\end{array}$ & $\begin{array}{c}\text { De } \\
\text { novo/WE } \\
\text { S }\end{array}$ & $\begin{array}{c}\text { Inherited } \\
\text { (mat)/W } \\
\text { ES }\end{array}$ & $\begin{array}{c}\text { Inherited } \\
\text { (pat)/WE } \\
\text { S }\end{array}$ & $\begin{array}{c}\text { De } \\
\text { novo/W } \\
\text { ES }\end{array}$ & $\begin{array}{c}\text { De } \\
\text { novo/W } \\
\text { ES }\end{array}$ & $\begin{array}{c}\text { De } \\
\text { novo/W } \\
\text { ES }\end{array}$ \\
\hline $\begin{array}{c}\text { Age at last } \\
\text { evaluation } \\
\#\end{array}$ & $\begin{array}{c}14 y, \\
\text { deceased } \\
\text { (respirato } \\
\text { ry } \\
\text { complica } \\
\text { tions of } \\
\text { status } \\
\text { epileptic } \\
\text { us) } \\
\end{array}$ & $\begin{array}{c}4 \mathrm{~m}, \\
\text { deceased } \\
\text { (respirat } \\
\text { ory } \\
\text { complic } \\
\text { ations of } \\
\text { status } \\
\text { epileptic } \\
\text { us) }\end{array}$ & $12 y$ & $3 y$ & $7 y$ & $32 y$ & $28 y$ & $\begin{array}{c}10 \mathrm{~d}, \\
\text { deceased } \\
\text { (respirat } \\
\text { ory } \\
\text { complica } \\
\text { tions of } \\
\text { status } \\
\text { epileptic } \\
\text { us) }\end{array}$ & $\begin{array}{c}2 \mathrm{y} 6 \mathrm{~m}, \\
\text { decease } \\
\mathrm{d} \\
\text { (intercur } \\
\text { rent } \\
\text { respirato } \\
\text { ry } \\
\text { infection } \\
\text { ) }\end{array}$ \\
\hline $\begin{array}{c}\text { Prenatal/p } \\
\text { erinatal } \\
\text { findings }\end{array}$ & $\begin{array}{l}\text { Unremar } \\
\text { kable }\end{array}$ & $\begin{array}{l}\text { Prenatal } \\
\text { US at } \\
32 \mathrm{w}: \\
\text { microgn } \\
\text { athia, } \\
\text { prefront } \\
\text { al } \\
\text { edema, } \\
\text { reduced } \\
\text { fetal } \\
\text { moveme } \\
\text { nts. } \\
\text { Apgar } \\
2 / 5 / 7\end{array}$ & $\begin{array}{l}\text { Unremar } \\
\text { kable }\end{array}$ & $\begin{array}{l}\text { Unremar } \\
\text { kable }\end{array}$ & $\begin{array}{l}\text { Unremar } \\
\text { kable }\end{array}$ & $\begin{array}{c}\text { Unremar } \\
\text { kable }\end{array}$ & $\begin{array}{l}\text { Unremar } \\
\text { kable }\end{array}$ & $\begin{array}{l}\text { Unremar } \\
\text { kable }\end{array}$ & $\begin{array}{c}\text { Born at } \\
\text { term by } \\
\text { Caesare } \\
\text { an } \\
\text { section } \\
\text { because } \\
\text { of } \\
\text { maternal } \\
\text { bleeding }\end{array}$ \\
\hline $\begin{array}{c}\text { Age at } \\
\text { seizure } \\
\text { onset/ } \\
\text { type/severi } \\
\text { ty }\end{array}$ & $\begin{array}{l}2 \mathrm{~m} / \text { focal } \\
\text { clonic- } \\
\text { tonic, } \\
\text { tonic } \\
\text { posturing } \\
\text { and } \\
\text { sweating, } \\
\text { dyscogni } \\
\text { tive with } \\
\text { nystagm } \\
\text { us,episod } \\
\text { es of } \\
\text { status } \\
\text { epileptic } \\
\text { us/severe }\end{array}$ & $\begin{array}{c}9 \mathrm{~d} / \\
\text { migratin } \\
\mathrm{g} \text { focal } \\
\text { seizures } \\
\text { with } \\
\text { apnea, } \\
\text { hyperton } \\
\text { us, and } \\
\text { eye } \\
\text { deviatio } \\
\text { n,super- } \\
\text { refractor } \\
\text { y status } \\
\text { epileptic } \\
\text { us/sever } \\
\text { e }\end{array}$ & $\begin{array}{l}2 y / \text { tonic } \\
\text { seizures } \\
\text { with } \\
\text { cyanosis/ } \\
\text { severe }\end{array}$ & $\begin{array}{c}\text { At birth/ } \\
\text { multifoca } \\
1 \\
\text { seizures, } \\
\text { eye } \\
\text { deviation } \\
\text {, tonic } \\
\text { posturing } \\
\text { episodes } \\
\text { of status } \\
\text { epileptic } \\
\text { us/severe }\end{array}$ & $\begin{array}{c}\text { 3y/gener } \\
\text { alised } \\
\text { tonic } \\
\text { during } \\
\text { sleep/mo } \\
\text { derately } \\
\text { severe }\end{array}$ & $\begin{array}{c}\text { No } \\
\text { seizures }\end{array}$ & $\begin{array}{c}1 \mathrm{~m} / \\
\text { hypotoni } \\
\text { a and } \\
\text { cyanosis; } \\
\text { 3y/absen } \\
\text { ce with } \\
\text { eyelid } \\
\text { myoclon } \\
\text { ia/severe }\end{array}$ & $\begin{array}{c}\text { At } \\
\text { birth/neo } \\
\text { natal } \\
\text { seizures } \\
\text { with } \\
\text { apnea, } \\
\text { cyanosis } \\
\text { and } \\
\text { hyperton } \\
\text { us, } \\
\text { evolving } \\
\text { to status } \\
\text { epileptic } \\
\text { us/severe }\end{array}$ & $\begin{array}{c}2 \mathrm{w} / \text { seve } \\
\text { re } \\
\text { epilepsy } \\
\text { (semiolo } \\
\text { gy } \\
\text { unspecif } \\
\text { ied) }\end{array}$ \\
\hline
\end{tabular}




\begin{tabular}{|c|c|c|c|c|c|c|c|c|c|}
\hline & \multicolumn{9}{|c|}{$A T P 1 A 3$, heterozygous } \\
\hline $\begin{array}{l}\text { Patient } \\
\text { Nr/sex }\end{array}$ & $10 / M$ & $11 / F$ & $12 / \mathrm{M}$ & $13 / M$ & 14/M & $15 / F$ & $16 / M$ & $17 / F$ & $18 / F$ \\
\hline $\begin{array}{c}\text { Interictal } \\
\text { EEG }\end{array}$ & $\begin{array}{c}\text { Slow } \\
\text { backgrou } \\
\text { nd, } \\
\text { multifoca } \\
1 \\
\text { discharge } \\
\text { s }\end{array}$ & $\begin{array}{c}\text { Burst } \\
\text { suppress } \\
\text { ion and } \\
\text { multifoc } \\
\text { al } \\
\text { discharg } \\
\text { es }\end{array}$ & $\begin{array}{l}\text { Multifoc } \\
\text { al } \\
\text { discharg } \\
\text { es }\end{array}$ & $\begin{array}{c}\text { Multifoc } \\
\text { al } \\
\text { discharge } \\
\text { s }\end{array}$ & $\begin{array}{l}\text { Backgro } \\
\text { und with } \\
\text { beta } \\
\text { activity, } \\
\text { multifoc } \\
\text { al } \\
\text { discharg } \\
\text { es }\end{array}$ & $\begin{array}{c}\text { Right } \\
\text { temporal } \\
\text { slow and } \\
\text { sharp } \\
\text { wave } \\
\text { activity }\end{array}$ & $\begin{array}{c}\text { Focal } \\
\text { and } \\
\text { generalis } \\
\text { ed } \\
\text { discharg } \\
\text { es, photo } \\
\text { paroxys } \\
\text { mal } \\
\text { response }\end{array}$ & $\begin{array}{l}\text { Multifoc } \\
\text { al } \\
\text { discharg } \\
\text { es }\end{array}$ & $\begin{array}{c}\text { EEG } \\
\text { details } \\
\text { missing }\end{array}$ \\
\hline $\begin{array}{c}\text { Brain } \\
\text { MRI: } \\
\text { age/findin } \\
\text { gs }\end{array}$ & $\begin{array}{l}\text { 13y/mild } \\
\text { bilateral } \\
\text { frontote } \\
\text { mporal } \\
\text { atrophy }\end{array}$ & $\begin{array}{c}2 \mathrm{~m} / \text { diffu } \\
\text { se } \\
\text { polymicr } \\
\text { ogyria, } \\
\text { dilated } \\
\text { ventricle } \\
\text { s }\end{array}$ & $\begin{array}{c}\text { 8y/norm } \\
\text { al }\end{array}$ & $\begin{array}{l}\text { 1d/extens } \\
\text { ive, } \\
\text { bilateral, } \\
\text { perisylvi } \\
\text { an } \\
\text { predomin } \\
\text { ant, } \\
\text { polymicr } \\
\text { ogyria }\end{array}$ & $\begin{array}{c}\text { 1y and } \\
\text { 3y/bilate } \\
\text { ral } \\
\text { extensive } \\
\text { perisylvi } \\
\text { an } \\
\text { predomi } \\
\text { nant } \\
\text { polymicr } \\
\text { ogyria, } \\
\text { thick } \\
\text { corpus } \\
\text { callosum }\end{array}$ & $\begin{array}{c}30 y / \\
\text { right } \\
\text { perisylvi } \\
\text { an } \\
\text { polymicr } \\
\text { ogyria, } \\
\text { thick } \\
\text { corpus } \\
\text { callosum }\end{array}$ & $\begin{array}{c}3 \mathrm{~m}, 1 \mathrm{y}, \\
5 \mathrm{y}, 8 \mathrm{y}, \\
17 \mathrm{y} \text { and } \\
25 \mathrm{y} / \mathrm{bilat} \\
\text { eral } \\
\text { extensiv } \\
\mathrm{e} \\
\text { perisylvi } \\
\text { an } \\
\text { predomi } \\
\text { nant } \\
\text { polymicr } \\
\text { ogyria; } \\
\text { no signs } \\
\text { of } \\
\text { progressi } \\
\text { on }\end{array}$ & $\begin{array}{c}2 \mathrm{~d} / \\
\text { bilateral } \\
\text { extensiv } \\
\mathrm{e}, \\
\text { perisylvi } \\
\text { an } \\
\text { predomi } \\
\text { nant } \\
\text { polymicr } \\
\text { ogyria }\end{array}$ & $\begin{array}{c}2 \mathrm{~m} / \text { nor } \\
\mathrm{mal}\end{array}$ \\
\hline $\begin{array}{c}\text { Microceph } \\
\text { aly (OFC } \\
<-2 \text { SD) }\end{array}$ & No & $\begin{array}{c}\text { Yes, } \\
\text { congenit } \\
\text { al } \\
(-3 \mathrm{SD})\end{array}$ & No & $\begin{array}{c}\text { Yes, } \\
\text { postnatal } \\
(-2 \mathrm{SD})\end{array}$ & No & No & No & $\begin{array}{c}\text { Yes, } \\
\text { congenit } \\
\text { al (SD } \\
\text { not } \\
\text { available } \\
\text { ) }\end{array}$ & No \\
\hline $\begin{array}{c}\text { Other } \\
\text { Neurologi } \\
\text { cal } \\
\text { Features }\end{array}$ & $\begin{array}{l}\text { Profound } \\
\text { develop } \\
\text { mental } \\
\text { delay, } \\
\text { hypotoni } \\
\text { c- } \\
\text { dyskineti } \\
\text { c } \\
\text { quadripar } \\
\text { esis, } \\
\text { optic } \\
\text { atrophy }\end{array}$ & $\begin{array}{c}\text { Severe } \\
\text { develop } \\
\text { mental } \\
\text { delay, } \\
\text { hypotoni } \\
\text { c } \\
\text { quadripa } \\
\text { resis, } \\
\text { absent } \\
\text { ABR }\end{array}$ & $\begin{array}{c}\text { Severe } \\
\text { develop } \\
\text { mental } \\
\text { delay, } \\
\text { hypotoni } \\
\text { c } \\
\text { quadripa } \\
\text { resis }\end{array}$ & $\begin{array}{c}\text { Severe } \\
\text { develop } \\
\text { mental } \\
\text { delay, } \\
\text { severe } \\
\text { axial } \\
\text { hypotoni } \\
\text { a }\end{array}$ & $\begin{array}{c}\text { Severe } \\
\text { develop } \\
\text { mental } \\
\text { delay, } \\
\text { hypotoni } \\
\text { c } \\
\text { quadripa } \\
\text { resis, } \\
\text { central } \\
\text { apnoea }\end{array}$ & $\begin{array}{c}\text { Mild } \\
\text { intellectu } \\
\text { al } \\
\text { disability } \\
\text { and } \\
\text { dyspraxi } \\
\text { a, } \\
\text { migraine } \\
\text { with } \\
\text { somatose } \\
\text { nsory } \\
\text { aura }\end{array}$ & $\begin{array}{c}\text { Severe } \\
\text { intellectu } \\
\text { al } \\
\text { disability } \\
\text { d } \\
\text { dyskines } \\
\text { ia; } \\
\text { alternati } \\
\text { ng } \\
\text { hemipleg } \\
\text { ia } \\
\text { attacks } \\
\text { since age } \\
\text { 5m with } \\
\text { good } \\
\text { response } \\
\text { to } \\
\text { flunarizi } \\
\text { ne }\end{array}$ & $\begin{array}{l}\text { Irritabilit } \\
\text { y, limb } \\
\text { hyperton } \\
\text { us, } \\
\text { abnorma } \\
1 \\
\text { neonatal } \\
\text { reflexes }\end{array}$ & $\begin{array}{c}\text { Severe } \\
\text { develop } \\
\text { mental } \\
\text { delay, } \\
\text { dyskinet } \\
\text { ic } \\
\text { quadripa } \\
\text { resis, } \\
\text { central } \\
\text { apnoea }\end{array}$ \\
\hline
\end{tabular}




\begin{tabular}{|c|c|c|c|c|c|c|c|c|c|}
\hline & \multicolumn{9}{|c|}{$A T P 1 A 3$, heterozygous } \\
\hline $\begin{array}{l}\text { Patient } \\
\text { Nr/sex }\end{array}$ & $10 / M$ & $11 / F$ & $12 / \mathbf{M}$ & $13 / \mathrm{M}$ & $14 / M$ & $15 / F$ & $16 / \mathrm{M}$ & $17 / F$ & $18 / \mathbf{F}$ \\
\hline $\begin{array}{l}\text { Additional } \\
\text { Clinical } \\
\text { findings } \\
\text { and ECG }\end{array}$ & $\begin{array}{l}\text { Drug- } \\
\text { induced } \\
\text { (valproat } \\
\text { e) renal } \\
\text { Fanconi } \\
\text { syndrom } \\
\text { e; ECG: } \\
\text { normal }\end{array}$ & $\begin{array}{l}\text { Pierre- } \\
\text { Robin } \\
\text { sequenc } \\
\text { e; ECG: } \\
\text { normal }\end{array}$ & $\begin{array}{c}\text { No; } \\
\text { ECG: } \\
\text { normal }\end{array}$ & $\begin{array}{l}\text { Short QT } \\
\text { interval, } \\
\text { unusual } \\
\text { ST } \\
\text { segment } \\
\text { in } 12 \\
\text { lead } \\
\text { ECG, } \\
\text { with two } \\
\text { normal } \\
\text { Holter } \\
\text { ECGs }\end{array}$ & $\begin{array}{l}\text { Hypogen } \\
\text { etic right } \\
\text { lung } \\
\text { with } \\
\text { mild } \\
\text { right } \\
\text { ventricul } \\
\text { ar } \\
\text { hypertro } \\
\text { phy and } \\
\text { stomach } \\
\text { malrotati } \\
\text { on; } \\
\text { ECG: } \\
\text { normal }\end{array}$ & $\begin{array}{c}\text { No; ECG } \\
\text { unavaila } \\
\text { ble }\end{array}$ & $\begin{array}{c}\text { No; } \\
\text { ECG: } \\
\text { normal }\end{array}$ & $\begin{array}{l}\text { High } \\
\text { pitched } \\
\text { cry; } \\
\text { ECG: } \\
\text { normal }\end{array}$ & $\begin{array}{l}\text { No; } \\
\text { ECG: } \\
\text { normal }\end{array}$ \\
\hline $\begin{array}{l}\text { Novel/Ref. } \\
\text { (for } \\
\text { previously } \\
\text { reported } \\
\text { mutations) }\end{array}$ & $\begin{array}{l}\text { (Marzin } \\
\text { et al., } \\
\text { 2018) }\end{array}$ & Novel & Novel & $\begin{array}{c}\text { (Rosewic } \\
\text { h et al., } \\
\text { 2012) }\end{array}$ & Novel & Novel & $\begin{array}{c}\text { (Panagio } \\
\text { takaki et } \\
\text { al., } \\
\text { 2015) }\end{array}$ & Novel & Novel \\
\hline
\end{tabular}


Table 1 (continues)

\begin{tabular}{|c|c|c|c|c|c|}
\hline & \multicolumn{4}{|c|}{$A T P 1 A 3$, heterozygous } & $\begin{array}{c}A T P 1 A 2 \\
\text { homozygous }\end{array}$ \\
\hline Patient $\mathrm{Nr} / \mathrm{sex}$ & 19/M & $20 / \mathbf{F}$ & $21 / F$ & $22 / F$ & $23 / F$ \\
\hline $\begin{array}{c}\text { Mutation } \\
\text { (protein)/abbrevi } \\
\text { ation }\end{array}$ & $\begin{array}{l}\text { p.(Asp992dup)/ } \\
\text { A3-D992dup }\end{array}$ & $\begin{array}{c}\text { p.(Ser361Pro)** } \\
\text { /A3-S361P }\end{array}$ & $\begin{array}{c}\text { p. }(\text { Phe } 857 \mathrm{del}) / \\
\text { A3-F857del }\end{array}$ & $\begin{array}{l}\text { p.(Leu924Pro)/ } \\
\text { A3-L924P }\end{array}$ & $\begin{array}{l}\text { p.(Arg279Glyfs*4) } \\
\text { /A2-R279Gfs*4 }\end{array}$ \\
\hline $\begin{array}{l}\text { Inherited/De } \\
\text { novo/type of } \\
\text { analysis }\end{array}$ & De novo/WES & De novo/WES & $\begin{array}{c}\text { De novo } \\
\text { (mosaic)/WES }\end{array}$ & De novo/WES & $\begin{array}{c}\text { Inherited } \\
\text { (biparental)/WES }\end{array}$ \\
\hline $\begin{array}{l}\text { Age at last } \\
\text { evaluation }\end{array}$ & $\begin{array}{l}3 \mathrm{w} \text {, deceased } \\
\text { (progressive } \\
\text { worsening of } \\
\text { the general } \\
\text { conditions } \\
\text { during status } \\
\text { epilepticus) }\end{array}$ & $7 \mathrm{~m} 12 \mathrm{~m}$ & $18 \mathrm{y}$ & $4 y 7 m$ & $\begin{array}{l}1 \mathrm{~d}, \text { deceased } \\
\text { (progressive } \\
\text { respiratory } \\
\text { insufficiency) }\end{array}$ \\
\hline $\begin{array}{c}\text { Prenatal/perinata } \\
\text { I findings }\end{array}$ & Unremarkable & Unremarkable & Unremarkable & $\begin{array}{l}\text { Delivery } \\
\text { complicated by } \\
\text { meconium } \\
\text { aspiration }\end{array}$ & \begin{tabular}{|} 
First trimester: \\
increased nuchal \\
translucency; \\
second trimester: \\
polyhydramnios, \\
IUGR, fetal \\
hydrops, \\
microcephaly, \\
clenched hands, \\
and abnormal fetal \\
movements \\
consistent with \\
seizure activity \\
\end{tabular} \\
\hline $\begin{array}{c}\text { Age at seizure } \\
\text { onset/ } \\
\text { type/severity }\end{array}$ & $\begin{array}{l}\text { At birth/focal } \\
\text { motor with } \\
\text { tonic posturing, } \\
\text { evolving to } \\
\text { status } \\
\text { epilepticus/seve } \\
\text { re }\end{array}$ & $\begin{array}{l}3 \mathrm{~m} / \text { focal clonic, } \\
\text { bilateral tonic } \\
\text { posturing with } \\
\text { apnea/controlled } \\
\text { since age } 5 \mathrm{~m}\end{array}$ & $\begin{array}{c}\text { Day } \\
\text { 1/hiccoughs, } \\
\text { stiffening and } \\
\text { abducting; } \\
\text { 2m/left- focal; } \\
\text { 2y/ tonic, oral } \\
\text { automatisms, } \\
\text { apnea, severe } \\
\text { clusters } \\
\text { 30/day, often } \\
\text { evolving into } \\
\text { spasms; } \\
\text { 5y/tonic, } \\
\text { atonic, } \\
\text { migrating } \\
\text { focal seizures, } \\
\text { focal impaired } \\
\text { awareness; } \\
\text { 19y/focal, } \\
\text { tonic, } \\
\text { spasms/severe }\end{array}$ & $\begin{array}{c}\text { 6w/ multifocal } \\
\text { seizures; } \\
\text { 4y/epileptic } \\
\text { spasms. For } \\
\text { most of life } \\
\text { daily use of } \\
\text { emergency } \\
\text { antiseizure } \\
\text { medications } \\
\text { and bag+mask } \\
\text { ventilation } \\
\text { because of } \\
\text { compromising } \\
\text { focal seizures } \\
\text { or focal status } \\
\text { epilepticus/sev } \\
\text { ere }\end{array}$ & $\begin{array}{l}\text { At birth/neonatal } \\
\text { seizures }\end{array}$ \\
\hline
\end{tabular}




\begin{tabular}{|c|c|c|c|c|c|}
\hline & \multicolumn{4}{|c|}{$A T P 1 A 3$, heterozygous } & $\begin{array}{c}A T P 1 A 2, \\
\text { homozygous }\end{array}$ \\
\hline Patient $\mathrm{Nr} / \mathrm{sex}$ & $19 / \mathrm{M}$ & $20 / \mathbf{F}$ & $21 / F$ & $22 / F$ & $23 / F$ \\
\hline Interictal EEG & NA & $\begin{array}{c}3-7 \mathrm{~m} / \mathrm{slow} \\
\text { background, } \\
\text { more prominent } \\
\text { on the right }\end{array}$ & $\begin{array}{c}\text { 8y/poorly } \\
\text { organised } \\
\text { background, } \\
\text { bilateral } \\
\text { asynchronous } \\
\text { epileptiform } \\
\text { discharges; } \\
18 \mathrm{y} / \\
\text { multifocal } \\
\text { discharges } \\
\end{array}$ & $\begin{array}{c}\text { 10w/normal } \\
\text { background, } \\
\text { multifocal } \\
\text { discharges; 1- } \\
\text { 4y/slow } \\
\text { background } \\
\text { multifocal } \\
\text { discharges }\end{array}$ & NA \\
\hline $\begin{array}{l}\text { Brain MRI: } \\
\text { age/findings }\end{array}$ & $\begin{array}{l}\text { 1d/abnormality } \\
\text { of thalamus } \\
\text { morphology, } \\
\text { bilateral, } \\
\text { polymicrogyria } \\
\text { more severe } \\
\text { anteriorly }\end{array}$ & $\begin{array}{c}3 \mathrm{~m} / \text { bilateral } \\
\text { perisylvian } \\
\text { polymicrogyria }\end{array}$ & $\begin{array}{c}5 y / \text { mild } \\
\text { atrophy with } \\
\text { small right } \\
\text { hippocampus; } \\
\text { bilateral } \\
\text { anterior } \\
\text { delayed } \\
\text { myelination }\end{array}$ & $2 \mathrm{~m} /$ normal & $\begin{array}{l}\text { MRI not available; } \\
\text { Cranial CT: scan } \\
\text { ventriculomegaly, } \\
\text { diffuse } \\
\text { calcifications and } \\
\text { poor gyral } \\
\text { formation }\end{array}$ \\
\hline $\begin{array}{l}\text { Microcephaly } \\
\text { (OFC <-2SD) }\end{array}$ & $\begin{array}{l}\text { Yes, postnatal } \\
\text { (SD not } \\
\text { available) } \\
\end{array}$ & $\begin{array}{l}\text { Yes, congenital } \\
\quad(-3.2 \mathrm{SD})\end{array}$ & No & $\begin{array}{l}\text { Yes, postnatal } \\
\quad(-2.6 \mathrm{SD})\end{array}$ & Yes, congenital \\
\hline $\begin{array}{c}\text { Other } \\
\text { Neurological } \\
\text { Features }\end{array}$ & $\begin{array}{c}\text { Hypotonic } \\
\text { quadriparesis }\end{array}$ & $\begin{array}{l}\begin{array}{c}\text { Axial hypotonia, } \\
\text { quadriparesis; } \\
\text { alternating }\end{array} \\
\begin{array}{c}\text { hemiplegia } \\
\text { attacks since age }\end{array} \\
\frac{\text { 7m, flunarizine }}{\text { started at } 12 \mathrm{~m}} \\
\underline{\text { months }}\end{array}$ & $\begin{array}{c}\text { Severe } \\
\text { intellectual } \\
\text { disability with } \\
\text { regression: } \\
\text { babbled at } \\
12 \mathrm{~m} \text { and } \\
\text { ceased at } 5 \mathrm{y}, \\
\text { walked at } 4 \mathrm{y} \\
\text { but became } \\
\text { unstable; } \\
\text { reluctant to } \\
\text { walk and non- } \\
\text { verbal at } 18 \mathrm{y}\end{array}$ & $\begin{array}{c}\text { Severe- } \\
\text { profound } \\
\text { developmental } \\
\text { delay, not able } \\
\text { to grasp, } \\
\text { inconsistent } \\
\text { visual } \\
\text { attention, no } \\
\text { head control, } \\
\text { no oral feeding }\end{array}$ & $\begin{array}{l}\text { No spontaneous } \\
\text { respiratory drive; } \\
\text { generalised four } \\
\text { limb contractures }\end{array}$ \\
\hline $\begin{array}{c}\text { Additional } \\
\text { Clinical findings } \\
\text { and ECG }\end{array}$ & $\begin{array}{l}\text { No; ECG: } \\
\text { normal }\end{array}$ & $\begin{array}{l}\text { Congenital hip } \\
\text { dysplasia; ECG: } \\
\text { normal }\end{array}$ & $\begin{array}{l}\text { Constipation, } \\
\text { PEG, } \\
\text { fundoplication, } \\
\text { precocious } \\
\text { puberty, mild } \\
\text { scoliosis, } \\
\text { divergent } \\
\text { strabismus; } \\
\text { ECG: normal } \\
\end{array}$ & $\begin{array}{c}\text { Gastrostomy } \\
\text { fed, } \\
\text { constipation, } \\
\text { sialorrhea, } \\
\text { osteopenia } \\
\text { with pathologic } \\
\text { fracture, hip } \\
\text { dysplasia; } \\
\text { ECG: normal } \\
\end{array}$ & $\begin{array}{l}\text { Hypertelorism, } \\
\text { proptotic eyes with } \\
\text { shallow orbits, } \\
\text { periorbital edema, } \\
\text { high arched palate } \\
\text { and a short neck }\end{array}$ \\
\hline $\begin{array}{c}\text { Novel/Ref. (for } \\
\text { previously } \\
\text { reported } \\
\text { mutations) }\end{array}$ & Novel & Novel & $\begin{array}{c}\text { (Retterer et al., } \\
\text { 2016) }\end{array}$ & $\begin{array}{l}\text { (Arystarkhova } \\
\text { et al., 2019) }\end{array}$ & $\begin{array}{c}\text { (Monteiro et al., } \\
2020)\end{array}$ \\
\hline
\end{tabular}


Table 1 - Clinical features in our cohort. Patients 1-19 are ordered by c.DNA. The position of Patients 20-22 at the end of the table reflects their inclusion in this series at a late stage. ${ }^{\text {For }}$ deceased patients, the cause of death is reported in brackets. The c.DNA positions refer to the reference transcripts NM_000702.4 (for ATP1A2) and NM_152296.5 (for ATP1A3). Abbreviations and symbols: **, recurrent mutation identified in $>1$ unrelated patients from this cohort; d, days; w, weeks; m, months; y, years; ABR, auditory brainstem response; ADHD, attention deficit hyperactivity disorder; ID, intellectual disability; IPS, intermittent photic stimulation; IUGR, intrauterine growth restriction; NA, not available/not applicable; PEG, percutaneous endoscopic gastrostomy; OFC, occipital frontal circumference; TC, tonic-clonic; TGP, targeted-gene panel; US, ultrasound; WES, whole-exome sequencing; WGS, whole-genome sequencing. 
Table 2

\begin{tabular}{|c|c|c|c|c|c|c|c|c|c|c|c|c|c|c|c|c|c|}
\hline \multicolumn{3}{|c|}{ Patient/Mutation } & \multicolumn{5}{|c|}{ Phenotype } & \multicolumn{10}{|c|}{ Location/Function } \\
\hline $\operatorname{Pt} N$ & Gene & Variant & $\begin{array}{l}\text { Died } \\
0-3 y\end{array}$ & DD/ID & MIC (subtype) & PMG & $\underline{\mathbf{A H}}$ & $\begin{array}{l}\text { Struct } \\
\text { ural } \\
\text { locati } \\
\text { on }\end{array}$ & $\begin{array}{c}\text { Cell } \\
\text { surviva } \\
\text { l in } \\
\text { culture } \\
\text { (under } \\
\text { ouabai } \\
\text { n } \\
\text { selectio } \\
\text { n) }\end{array}$ & $\begin{array}{l}\text { Phosph } \\
\text { o- } \\
\text { rylatio } \\
\text { n }\end{array}$ & $\begin{array}{c}\text { Ma } \\
\text { X } \\
\text { TO } \\
\text { rat } \\
\text { e }\end{array}$ & $\begin{array}{c}\text { NKA } \\
\text { activity/ } \\
\text { mg } \\
\text { membra } \\
\text { ne } \\
\text { protein }\end{array}$ & $\begin{array}{c}\mathrm{Na}^{+} \\
\text {affinity }\end{array}$ & $\begin{array}{l}\mathrm{Na}^{+} / \mathrm{K}^{+} \\
\text {selectiv } \\
\text { ity }\end{array}$ & $\begin{array}{c}\mathbf{K}^{+} \\
\text {affinity }\end{array}$ & $\begin{array}{c}\text { Conformati } \\
\text { onal change } \\
\text { E1-E2 }\end{array}$ & $\begin{array}{c}\text { Conformatio } \\
\text { nal change } \\
\text { E1P-E2P }\end{array}$ \\
\hline
\end{tabular}

\begin{tabular}{|c|c|c|c|c|c|c|c|c|c|c|c|c|c|c|c|c|c|}
\hline 2 & $\begin{array}{c}\text { ATP1 } \\
\text { A2 } \\
\end{array}$ & $\begin{array}{c}\mathrm{A} 2- \\
\mathrm{C} 341 \mathrm{~F} \\
\end{array}$ & No & SEV & No & No & $\underline{\mathrm{No}}$ & M4 & No & $10-15 \%$ &.. &.. &.. &.$\cdot$ &.. &.. &. \\
\hline 3 & $\begin{array}{c}\text { ATP1 } \\
\text { A2 } \\
\end{array}$ & $\begin{array}{c}\text { A2- } \\
\text { G366A }\end{array}$ & Yes & SEV & No & No & $\underline{\text { No }}$ & $\mathrm{P}$ & No & $61 \%$ &.. &.. & $\begin{array}{l}\downarrow 3-5 \\
\text { fold }\end{array}$ &.. & $\begin{array}{l}\downarrow>15 \\
\text { fold }\end{array}$ &.. & $\begin{array}{c}\text { shift } \\
\mathrm{E} 1 \mathrm{P} \rightarrow \rightarrow \mathrm{E} 2 \mathrm{P}\end{array}$ \\
\hline 4 & $\begin{array}{c}\text { ATP1 } \\
\text { A2 }\end{array}$ & $\begin{array}{c}\text { A2- } \\
\text { G366A }\end{array}$ & Yes & SEV & $\begin{array}{c}\text { MIC } \\
\text { (postnatal)* }\end{array}$ & No & $\underline{\text { No }}$ & $\mathrm{P}$ & No & $61 \%$ &.. &.. & $\begin{array}{l}\downarrow 3-5 \\
\text { fold }\end{array}$ &.. & $\begin{array}{l}\downarrow>15 \\
\text { fold }\end{array}$ &.$\cdot$ & $\begin{array}{c}\text { shift } \\
\mathrm{E} 1 \mathrm{P} \rightarrow \rightarrow \mathrm{E} 2 \mathrm{P}\end{array}$ \\
\hline 7 & $\begin{array}{c}\text { ATP1 } \\
\text { A3 } \\
\end{array}$ & $\begin{array}{c}\text { A3- } \\
\text { L292R }\end{array}$ & Yes & SEV & $\begin{array}{c}\text { MIC } \\
\text { (postnatal)** }\end{array}$ & Yes & $\underline{\mathrm{No}}$ & M3 & No & $61 \%$ &.. &.. & $\begin{array}{l}\downarrow 3-5 \\
\text { fold }\end{array}$ &.. & $\begin{array}{l}\downarrow>15 \\
\text { fold }\end{array}$ &.. &.. \\
\hline 8 & $\begin{array}{c}\text { ATP1 } \\
\mathbf{A 3}\end{array}$ & $\begin{array}{c}\text { A3- } \\
\text { G316V }\end{array}$ & No & SEV & No & No & $\underline{\text { No }}$ & M4 & No & $70 \%$ & .. & .. & $\uparrow 4$ fold &.. & $\begin{array}{c}\downarrow>15 \\
\text { fold }\end{array}$ &.. &.. \\
\hline 9 & $\begin{array}{c}\text { ATP1 } \\
\text { A3 } \\
\end{array}$ & A3-S361P & No & MOD & $\begin{array}{c}\text { MIC } \\
\text { (postnatal)** }\end{array}$ & No & $\underline{\text { No }}$ & $\mathrm{P}$ & No & $10-15 \%$ &.. &.. &.. &. &.. &.. &.. \\
\hline 20 & $\begin{array}{c}\text { ATP1 } \\
\text { A3 }\end{array}$ & A3-S361P & No & NA & $\begin{array}{c}\text { MIC } \\
\text { (congenital)** }\end{array}$ & Yes & $\underline{\text { Yes }}$ & $\mathrm{P}$ & No & $10-15 \%$ &.. &.. &.. &.. & .. &.. &.. \\
\hline 11 & $\begin{array}{c}\text { ATP1 } \\
\text { A3 } \\
\end{array}$ & $\begin{array}{c}\text { A3- } \\
\text { K764del } \\
\end{array}$ & Yes & SEV & $\begin{array}{c}\text { MIC } \\
\text { (congenital)** }\end{array}$ & Yes & $\underline{\text { No }}$ & M5 & No & $10-15 \%$ &.. & .. &.. &.. &.. &.. &.. \\
\hline 12 & $\begin{array}{c}\text { ATP1 } \\
\text { A3 } \\
\end{array}$ & $\begin{array}{c}\text { A3- } \\
\text { P775R }\end{array}$ & No & SEV & No & No & $\underline{\text { No }}$ & M5 & No & $\sim 0 \%$ &.$\cdot$ &.. &.. &.. &.. &.. &.. \\
\hline 16 & $\begin{array}{c}\text { ATP1 } \\
\text { A3 } \\
\end{array}$ & $\begin{array}{c}\text { A3- } \\
\text { L888P } \\
\end{array}$ & No & SEV & No & Yes & $\underline{\text { Yes }}$ & L7-8 & No & $\sim 0 \%$ &.. &.. &.. &.. &.. &.. &.. \\
\hline 19 & $\begin{array}{c}\text { ATP1 } \\
\text { A3 }\end{array}$ & $\begin{array}{c}\text { A3- } \\
\text { D992dup }\end{array}$ & Yes & NA & $\begin{array}{c}\text { MIC } \\
\text { (postnatal) }\end{array}$ & Yes & $\underline{\text { No }}$ & M10 & No & $\sim 0 \%$ &.. &.. &.. &.. &.. &.. &.. \\
\hline & & & $\begin{array}{c}5 \text { of } \\
11\end{array}$ & $\begin{array}{l}8 \text { of } 9 \\
\text { SEV }\end{array}$ & 6 of 11 & $\begin{array}{c}5 \text { of } \\
11\end{array}$ & $\frac{2 \text { of }}{11}$ & & & & & & & & & & \\
\hline \multicolumn{18}{|c|}{ Group 2 - Variant does support cell survival in culture (this study; $N=5$ mutations in 6 patients) } \\
\hline 1 & $\begin{array}{c}\text { ATP1 } \\
\text { A2 }\end{array}$ & $\begin{array}{c}\text { A2- } \\
\text { I293M }\end{array}$ & No & MOD & No & No & $\underline{\text { No }}$ & M3 & Yes & $>50 \%$ & $\begin{array}{l}27 \\
\%\end{array}$ & $\sim 17 \%$ & $\begin{array}{l}\downarrow 3-5 \\
\text { fold }\end{array}$ & $\downarrow$ &.. & $\begin{array}{c}\text { shift } \\
\mathrm{E} 2 \rightarrow \rightarrow \mathrm{E} 1\end{array}$ & $\begin{array}{c}\text { shift } \\
\mathrm{E} 2 \mathrm{P} \rightarrow \rightarrow \mathrm{E} 1 \mathrm{P}\end{array}$ \\
\hline
\end{tabular}




\begin{tabular}{|c|c|c|c|c|c|c|c|c|c|c|c|c|c|c|c|c|c|}
\hline 5 & $\begin{array}{c}\text { ATP1 } \\
\text { A2 }\end{array}$ & $\begin{array}{c}\text { A2- } \\
\text { R593Q }\end{array}$ & No & No & No & No & $\underline{\text { No }}$ & $P$ & Yes & $>50 \%$ & $\begin{array}{l}42 \\
\% \\
\end{array}$ & $\sim 36 \%$ & $\begin{array}{l}\sim \text { wild- } \\
\text { type }\end{array}$ & $\downarrow$ &.. & shift E2 $\rightarrow$ E1 & $\begin{array}{c}\text { shift } \\
\mathrm{E} 2 \mathrm{P} \rightarrow \mathrm{E} 1 \mathrm{P}\end{array}$ \\
\hline 6 & $\begin{array}{c}\text { ATP1 } \\
\text { A2 } \\
\end{array}$ & $\begin{array}{c}\text { A2- } \\
\text { R908Q }\end{array}$ & No & MOD & $\begin{array}{c}\text { MIC } \\
\text { (postnatal)** }\end{array}$ & Yes & $\underline{\text { No }}$ & L7-8 & Yes & $\sim 26 \%$ & $\begin{array}{l}84 \\
\% \\
\end{array}$ & $\sim 22 \%$ & $\begin{array}{l}\sim \text { wild- } \\
\text { type }\end{array}$ &.. &.. & shift E1 $\rightarrow$ E2 &.. \\
\hline 14 & $\begin{array}{c}\text { ATP1 } \\
\text { A3 }\end{array}$ & $\begin{array}{c}\text { A3- } \\
\text { D887Y }\end{array}$ & No & SEV & No & Yes & $\underline{\text { No }}$ & L7-8 & Yes & $\sim 35 \%$ & $\begin{array}{l}95 \\
\% \\
\end{array}$ & $\sim 33 \%$ & $\begin{array}{l}\text { wild- } \\
\text { type }\end{array}$ & $\downarrow$ &.. & shift E2 $\rightarrow$ E1 &.. \\
\hline 15 & $\begin{array}{c}\text { ATP1 } \\
\text { A3 } \\
\end{array}$ & $\begin{array}{c}\text { A3- } \\
\text { D887Y } \\
\end{array}$ & No & MILD & No & Yes & $\underline{\text { No }}$ & L7-8 & Yes & $\sim 35 \%$ & $\begin{array}{l}95 \\
\% \\
\end{array}$ & $\sim 33 \%$ & $\begin{array}{l}\text { 〜wild- } \\
\text { type }\end{array}$ & $\downarrow$ &.. & shift E2 $\rightarrow$ E1 &.. \\
\hline \multirow[t]{2}{*}{18} & $\begin{array}{c}\text { ATP1 } \\
\text { A3 }\end{array}$ & $\begin{array}{c}\text { A3- } \\
\text { P972del }\end{array}$ & Yes & SEV & No & No & $\underline{\text { No }}$ & L9-10 & Yes & $\sim 35 \%$ & $\begin{array}{l}85 \\
\%\end{array}$ & $\sim 30 \%$ & $\begin{array}{l}\downarrow \\
\text { slightly }\end{array}$ & $\uparrow$ &.. & shift E1 $\rightarrow$ E2 & $\begin{array}{c}\text { shift } \\
\text { E2P } \rightarrow \text { E1P }\end{array}$ \\
\hline & & & $\begin{array}{c}1 \text { of } \\
6\end{array}$ & $\begin{array}{l}2 \text { of } 6 \\
\text { SEV }\end{array}$ & 1 of 6 & 3 of 6 & $\underline{0 \text { of } 6}$ & & & & & & & & & & \\
\hline
\end{tabular}

Table 2 - Schematic representation of the main clinical features in our cohort with structural location and functional effect of the corresponding mutations. We separated the 17 patients (14 mutations) for whom experimental data were available from this study into two groups based on COS-1 cell survival under ouabain selection pressure. The mutations are distributed across the following locations: phosphorylation domain (P), transmembrane helices (M3-10) and their intervening loops (L). Nine different aspect of the NKA pump function investigated by at least one of the different experimental approaches are showed. Abbreviations and symbols: $\mathrm{AH}$, alternating hemiplegia; DD/ID, developmental delay/intellectual disability; MIC, microcephaly $(*=-2 \mathrm{SD} ; * *=>-3 \mathrm{SD}$; no asterisk means there is microcephaly but $\mathrm{SD}$ are unknown); MOD, moderate; mos, mosaic; NA, not applicable/not available; PMG, polymicrogyria; Pt, patient; SEV, severe; TO, turnover; VAF, variant allele frequency. 


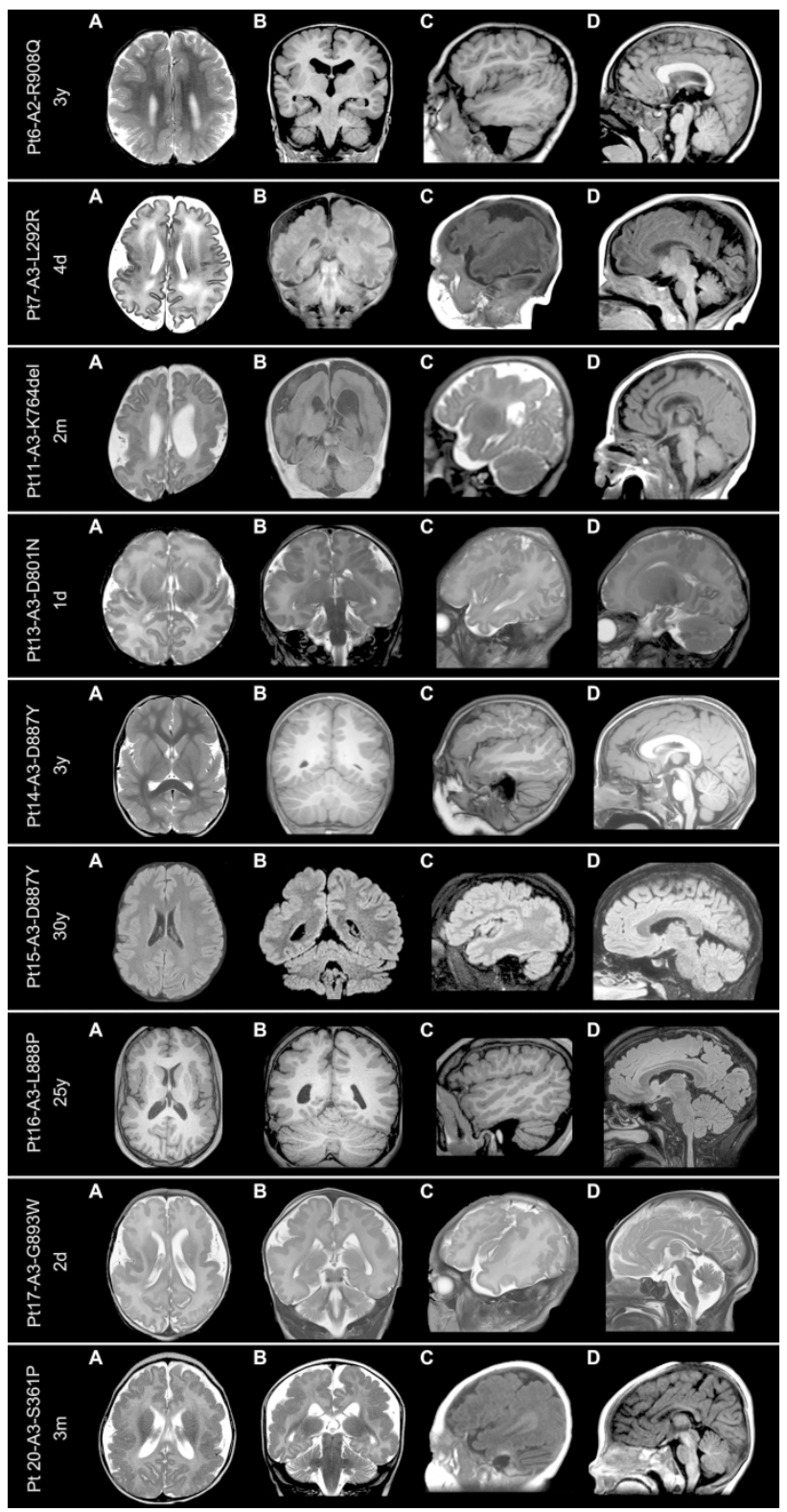

Figure 1 - Brain MRI in 9 patients with polymicrogyria. From top to bottom, representative brain MRI findings in Patients 6, 7, 11-17 and 20 with an indication of the mutations and age at which imaging was performed. For each patient four cuts are presented, which include axial (column A), coronal (column B), sagittal through the sylvian fissure (column C), and sagittal through the midline (column D) images. Sequences are at 1.5 to 3T and include T1-weighted, T2 weighted and fluid-attenuated inversion recovery (FLAIR) images. All patients have polymicrogyria with abnormal cortical infoldings and packed microgyri, combined with abnormal sulcation. A recurrent feature, present in all patients, is an abnormal sylvian fissure with thickened cortex, pronounced infolding and vertically oriented axis. These abnormalities are better visible in the A column (enlarged sylvian space), B column (infolding) and C column (cortical thickening and verticalized sylvian fissure). There are some differences in individual patients. In Patient 6, polymicrogyria is diffuse and more prominent posteriorly. In Patient 7, the cortical abnormality is asymmetrical, due to a smaller right hemisphere. In Patient 11 too, there is asymmetry with the right hemisphere being more severely affected and smaller. In Patients 13,14, 16 and 17, polymicrogyria is almost exclusively bilateral perisylvian, sparing the remaining cortex. In Patient 15 too, who is the mother of Patient 14, the cortical 
abnormality is perisylvian but unilateral only involving the right hemisphere. In Patient 17 , there is diffuse polymicrogyria with more severe perisylvian involvement. In line D, midline cuts show thickening of the corpus callosum in Patients 6, 14 and 15. There is some degree of vermian hypoplasia in Patients 7 and 17. 

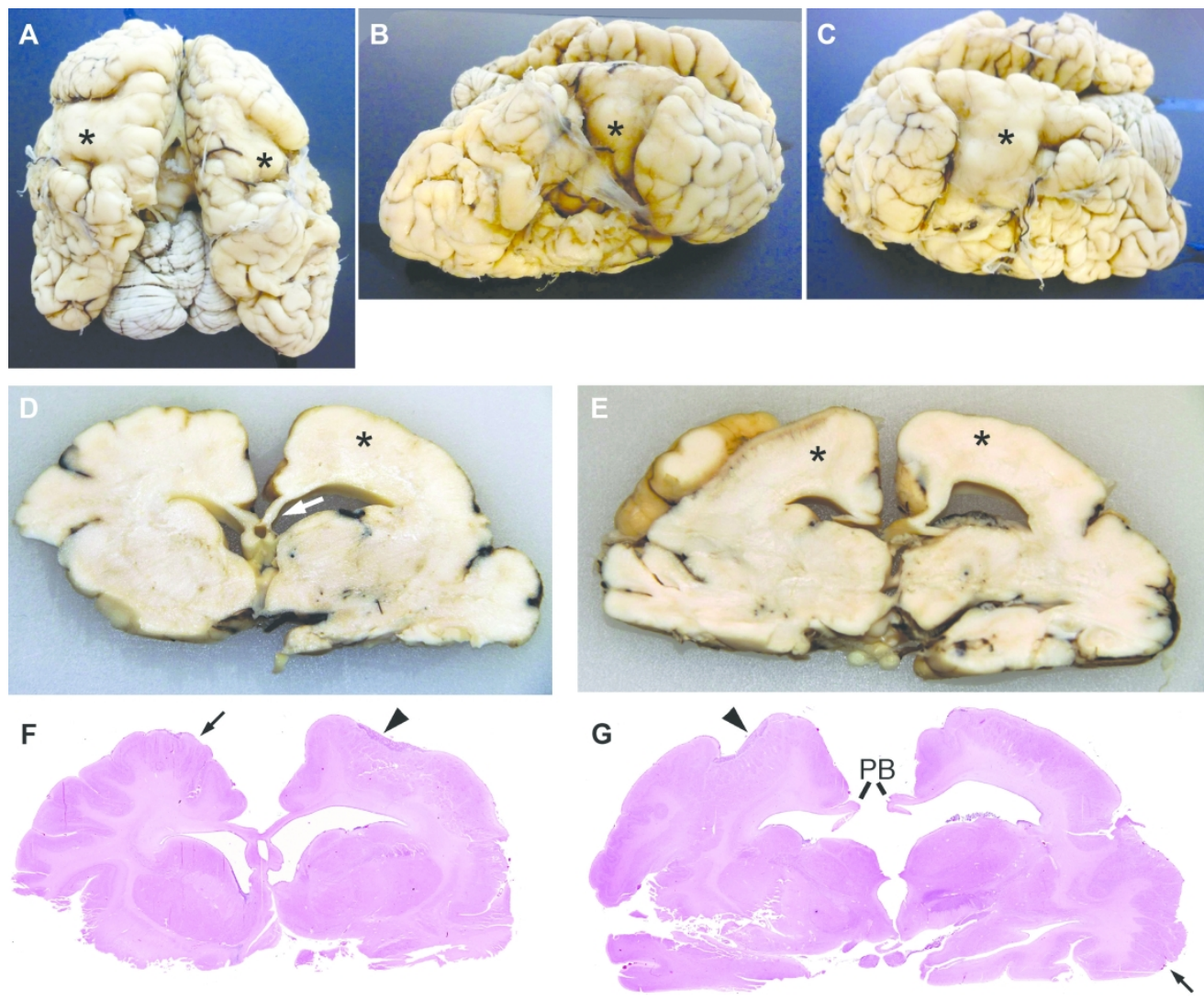

Figure 2 - Neuropathology of patient 11, age 4 months (A3-K764del). (A-C) Macroscopic views of the brain from posterior $(A)$, right (B), and left (C) sides demonstrated overall small cerebral hemispheres, with externally apparent pachygyria (asterisks) involving pericentral cortex bilaterally. (D, E) Coronal slices through anterior (D) and middle (E) regions of the hemispheres showed thick cortex corresponding to externally apparent pachygyria (asterisks), and thin but intact genu of the corpus callosum (white arrow, D).

$(F, G)$ Histology of the brain slices demonstrated leptomeningeal glioneuronal heterotopia (black arrowheads) covering cortex that appeared externally pachygyric, but histologically showed features of 4layered polymicrogyria, also seen in other cortical areas (black arrows). Callosal fibers did not cross the midline posteriorly, but instead formed Probst bundles (PB). 

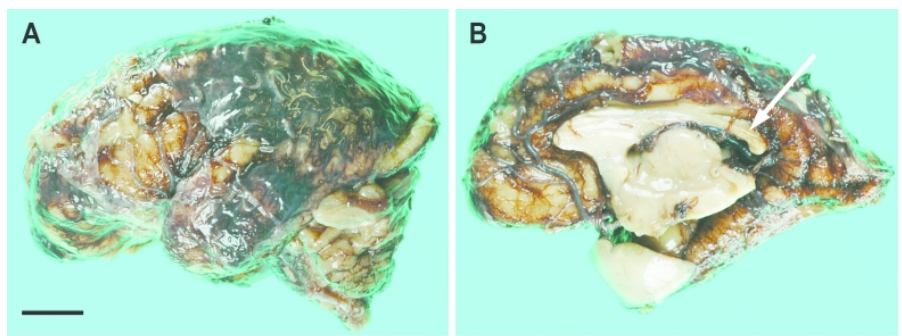

C

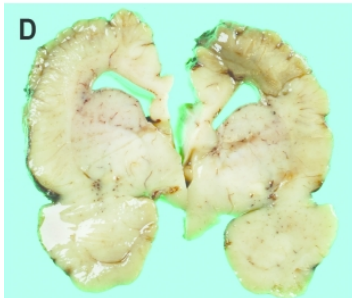

Exptory, $F$
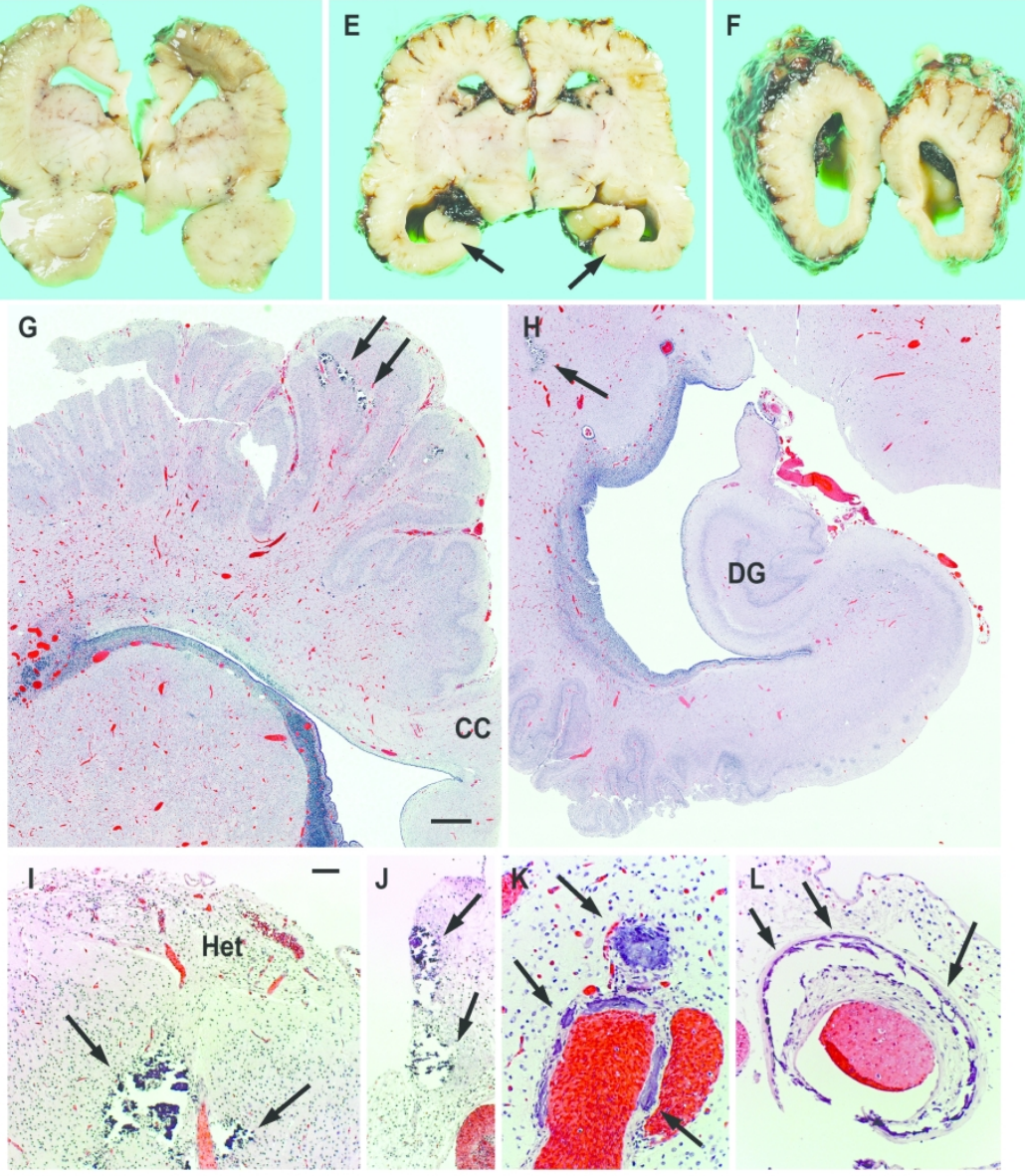

Neuropathology of patient 23, age 1 day (A2-R279Gfs4*, homozygous). Macroscopic brain abnormalities (AF). (A) Left brain, lateral view. Blood vessels were congested; the leptomeninges contained subarachnoid haemorrhage (agonal) and were adherent to the brain surface. (B) Right hemisphere, medial view. The corpus callosum was thin (white arrow). (C) Brainstem and right cerebellum, medial view. The cerebellum was moderately hypoplastic. (D, E) Coronal slices of brain, level of amygdala (D) and anterior hippocampus (E). The basal ganglia and thalamus were moderately hypoplastic; the cortex was diffusely polymicrogyric, except for the hippocampi (black arrows), which appeared relatively well-formed. (F) The occipital lobes (coronal slices) exhibited polymicrogyria and moderate colpocephaly. Histopathology (H\&E; G-N) (G) Frontal cortex, coronal section (midline right). The cortex was diffusely polymicrogyric, had an attached external rind of leptomeningeal glioneuronal heterotopia, and contained focal calcifications (arrows). The corpus

callosum (CC) was small, but crossed the midline. (H) Medial temporal lobe, coronal section. The hippocampus was well-formed, including the dentate gyrus (DG), while neocortex was polymicrogyric. Focal calcifications (arrows) were noted in deep white matter. (I) Leptomeningeal glioneuronal heterotopia (Het) covered much of the cortical surface. Note focal parenchymal calcifications (arrows). (J) Leptomeninges with 
focal calcifications (arrows). (K) Cortical blood vessel with mineralized walls (arrows). (L) Meningeal blood vessel with medial calcifications (arrows), and intimal hyperplasia possibly due to thrombosis and recanalization. Scale bars: (A) $1 \mathrm{~cm}$ for $(A-F) ;(G), 1 \mathrm{~mm}$ for $(\mathrm{G}, \mathrm{H})$; (I), $100 \mu \mathrm{m}$ for $(\mathrm{I}, \mathrm{J}) ; 50 \mu \mathrm{m}$ for $(\mathrm{K}, \mathrm{L})$. 


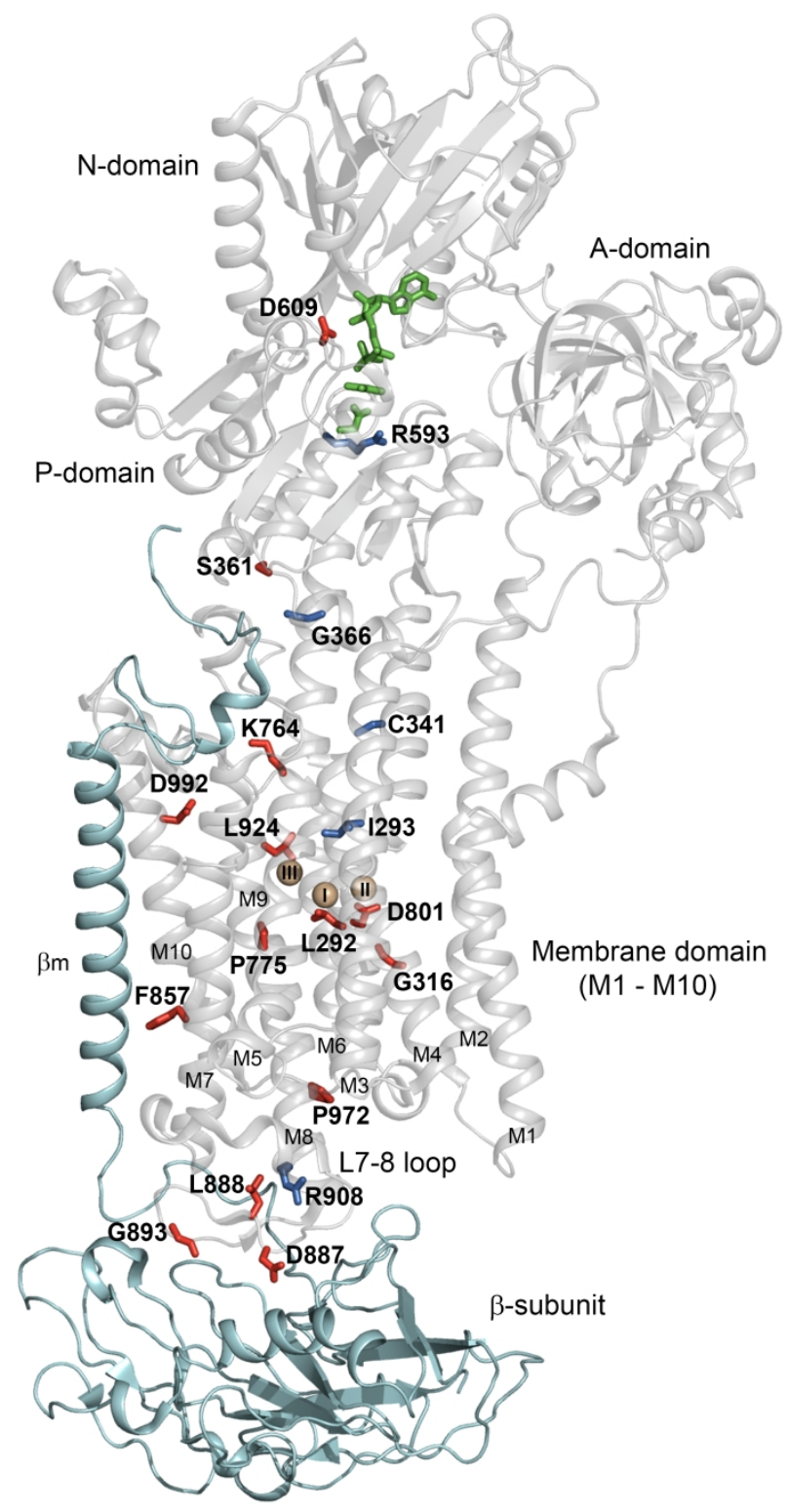

Figure 4: Overview of the structural location of ATP1A2 and ATP1A3 disease mutations in the cohort The NKA a-subunit consists of three major cytoplasmic domains A ("actuator"), N ("nucleotide-binding"), and $\mathrm{P}$ ("phosphorylation") linked to a membrane domain composed of ten transmembrane helices (M1-M10) with their intervening loops on both membrane sides. The $\beta$-subunit (PDB ID 3WGV, chain B, teal color) consists of a transmembrane helix $\beta \mathrm{m}$ and an extracellular domain that interacts with the a-subunit loop connecting M7 and M8 (L7-8 loop). Amino acid residues targeted by the mutations in the cohort are shown in blue (ATP1A2 mutations) and red (ATP1A3 mutations) in the structure of the ATP1A1 a-subunit in E1 form (PDB ID 3WGV, chain A, grey color) with $3 \mathrm{Na}+$ (golden spheres labelled I, II, and III according to common nomenclature) and ADP with phosphate analog AlF4 and the phosphorylated aspartate in green.

$99 \times 189 \mathrm{~mm}(600 \times 600 \mathrm{DPI})$ 

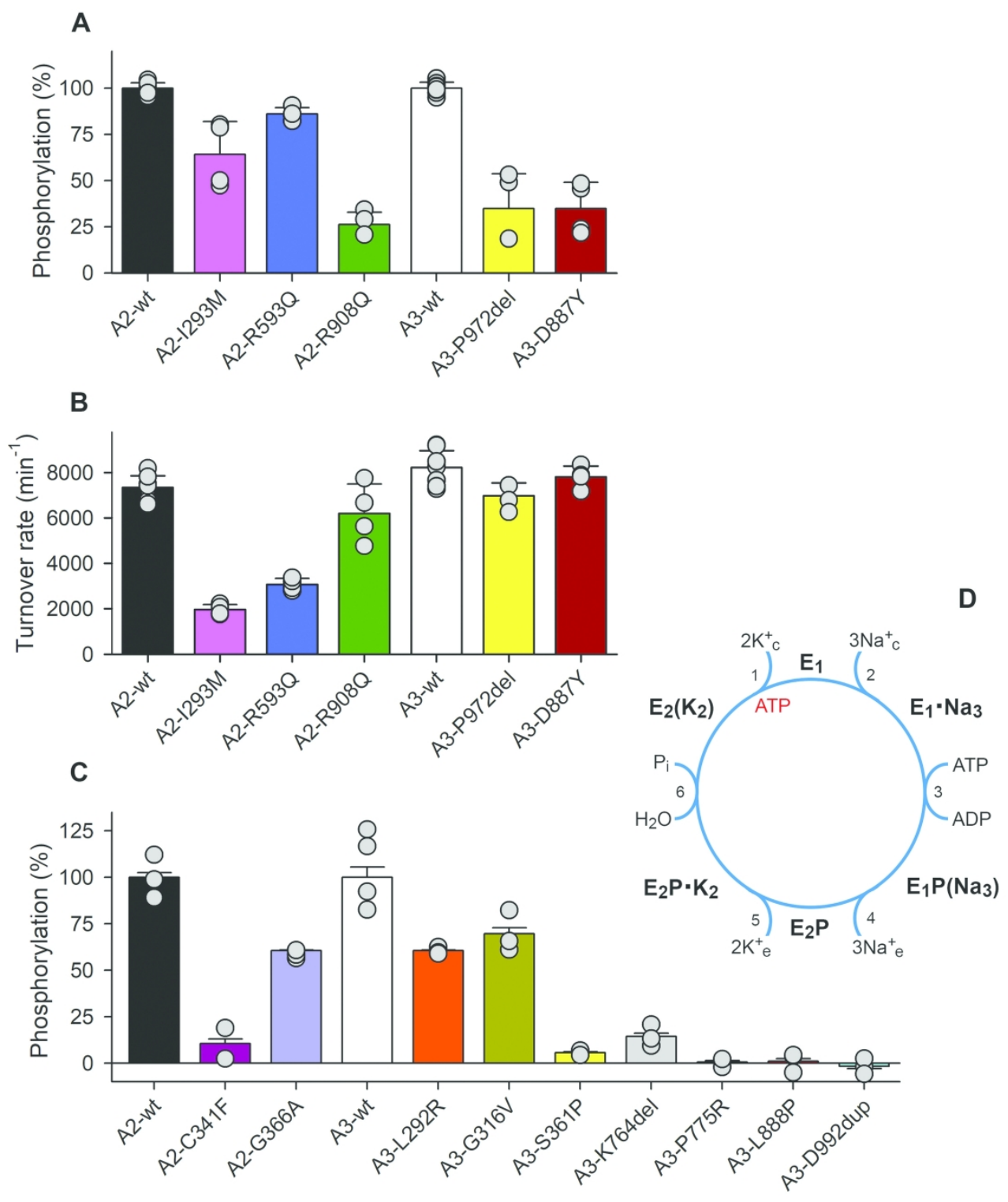

Figure 5: Relative phosphorylation/expression and turnover rate for ATPase activity with NKA reaction cycle(A) Phosphorylation level of stably expressed ATP1A2 and ATP1A3 wild type and mutants. The phosphorylation level (pmol/mg total plasma membrane protein) is shown in $\%$ of wild type.

Phosphorylation was determined under stoichiometric conditions, thus reflecting the expression level ("active site concentration"). (B) Turnover rate (min-1) determined by relating the maximum NKA activity to the phosphorylation level. (C) Phosphorylation level of transiently expressed ATP1A2 and ATP1A3 wild type and mutants determined as described for (A). The NKA activity per mg total plasma membrane protein, calculated by multiplying the turnover rate by the phosphorylation level, was $17 \%$ (A2-I293M), 36\% (A2R593Q), 22\% (A2-R908Q), 30\% (A3-P972del), and 33\% (A3-D887Y), relative to wild-type, indicating that the function of these mutants was substantially impaired despite their ability to support cell growth. (D) Reaction cycle of NKA. E1 and E1P are Na+ selective conformations, whereas E2 and E2P are K+ selective conformations. Phosphorylation by ATP (indicated by "P"). The cytoplasmic and extracellular sides are indicated by $\mathrm{c}$ and e, respectively, see detailed description in supplementary legend to figure 5

(Supplementary Materials) (Holm et al., 2016). 
A

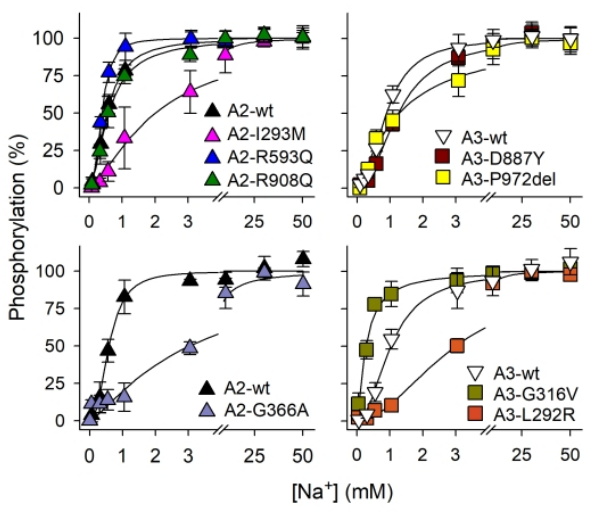

B

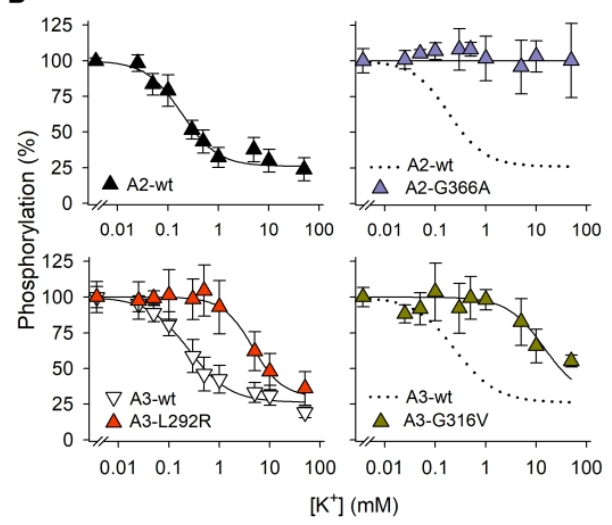

Figure 6: $\mathrm{Na}+$ and $\mathrm{K}+$ affinity of ATP1A2/A3 mutants

(A) $\mathrm{Na}+$ affinity determined from $\mathrm{Na}+$ activation of phosphorylation. The binding of three $\mathrm{Na}+$ to the $\mathrm{E} 1$ form activates phosphorylation from ATP. Phosphorylation was measured at the indicated concentrations of $\mathrm{Na}+$. Symbols with error bars represent mean \pm SD. Each line represents the best fit of a Hill equation. The apparent $\mathrm{Na}+$ affinities (K0.5 values) and Hill coefficients $(\mathrm{nH})$ were as follows. Stable expression (upper panels): A2-wt (wild-type), $534 \pm 52 \mu \mathrm{M}(\mathrm{nH}=2.0) ; \mathrm{A} 2-\mathrm{I} 293 \mathrm{M}, 2790 \pm 592 \mu \mathrm{M}(\mathrm{nH}=1.7) ; \mathrm{A} 2-\mathrm{R} 593 \mathrm{Q}$,

$384 \pm 24 \mu \mathrm{M}(\mathrm{nH}=2.6) ; \mathrm{A} 2-\mathrm{R} 908 \mathrm{Q}, 601 \pm 81 \mu \mathrm{M}(\mathrm{nH}=1.7) ; \mathrm{A} 3-\mathrm{wt}, 916 \pm 105 \mu \mathrm{M}(\mathrm{nH}=2.4) ; \mathrm{A} 3-$ $\mathrm{D} 887 \mathrm{Y}, 1260 \pm 93 \mu \mathrm{M}(\mathrm{nH}=2.1) ; \mathrm{A} 3-\mathrm{P} 972 \mathrm{del}, 1331 \pm 303 \mu \mathrm{M}(\mathrm{nH}=1.2)$. Transient expression (lower panels): A2-wt, $595 \pm 80 \mu \mathrm{M}(\mathrm{nH}=2.5) ; \mathrm{A} 2-\mathrm{G} 366 \mathrm{~A}, 3079 \pm 192 \mu \mathrm{M}(\mathrm{nH}=1.3) ; \mathrm{A} 3-\mathrm{wt}, 1120 \pm 127 \mu \mathrm{M}$ $(\mathrm{nH}=2.1) ; \mathrm{A3}-\mathrm{L} 292 \mathrm{R}, 3013 \pm 254 \mu \mathrm{M}(\mathrm{nH}=1.9) ; \mathrm{A} 3-\mathrm{G} 316 \mathrm{~V}, 272 \pm 47 \mu \mathrm{M}(\mathrm{nH}=1.3)$. The apparent $\mathrm{Na}+$ affinity was reduced 3- to 5-fold (K0.5 increased) for A2-I293M, A2-G366A, and A3-L292R, and increased 4fold for A3-G316V (K0.5 decreased). For A3-P972del, the cooperativity of $\mathrm{Na}+$ binding at the three sites was severely reduced (Hill coefficient 1.2 vs. 2.4 for the wild-type). (B) $\mathrm{K}+$ affinity determined from $\mathrm{K}+$ inhibition of phosphorylation. Accumulation of phosphoenzyme is inhibited by $\mathrm{K}+$, because $\mathrm{K}+$ activates dephosphorylation of E2P and competes with $\mathrm{Na}+$ for binding to $\mathrm{E} 1$ (cf. reaction cycle in Fig. 5).

Phosphorylation was measured at the indicated concentrations of $\mathrm{K}+$ on transiently expressed NKA enzyme. Symbols with error bars represent mean \pm SD. Each line represents the best fit of the Hill equation for inhibitory ligand binding (Nielsen et al., 2019). The apparent $\mathrm{K}+$ affinities (K0.5 values) were as follows: A2wt, $188 \pm 43 \mu \mathrm{M}$; A2-G366A, >100 mM; A3-wt, $281 \pm 63 \mu \mathrm{M}$; A3-L292R, $4570 \pm 700 \mu \mathrm{M} ; \mathrm{A3}-\mathrm{G} 316 \mathrm{~V},>10$ $\mathrm{mM}$. Hence, A3-L292R and A3-G316V showed 16- and >35-fold reduction of apparent K+ affinity, and A2G366A was totally insensitive to $\mathrm{K}+$.

$176 \times 76 \mathrm{~mm}(600 \times 600 \mathrm{DPI})$ 


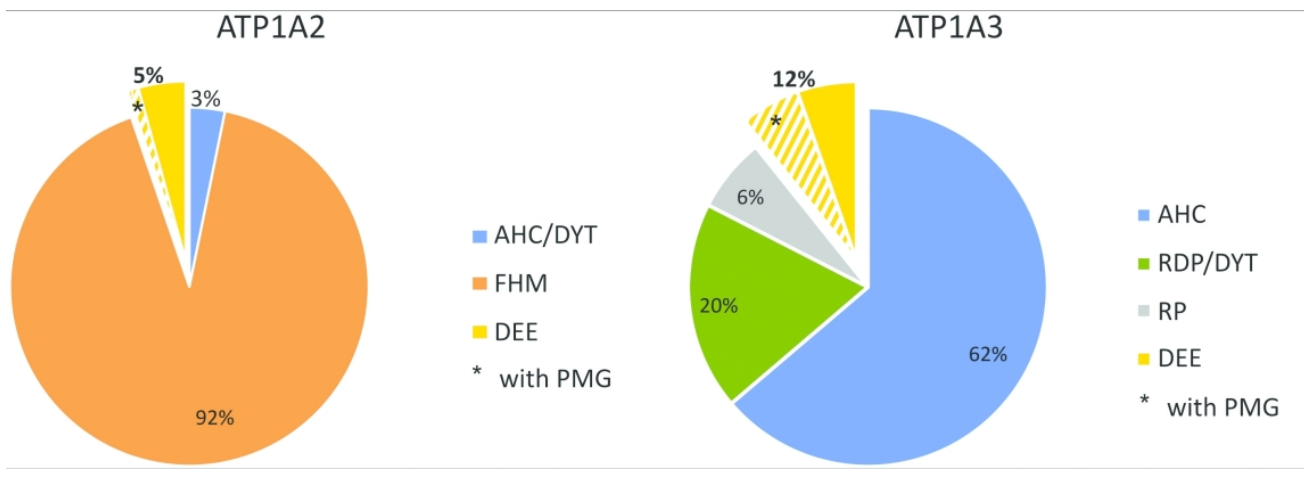

Figure 7 - Distribution of ATP1A2/A3 mutations across their associated phenotypes.

The pie charts show the distribution of heterozygous mutations of ATP1A2 (left) and ATP1A3 (right) across their associated phenotypes. We identified 92 ATP1A2 and 136 ATP1A3 heterozygous mutations in the Human Gene Mutation Database (https://portal.biobase-international.com), and stratified them based on the reported phenotypes, adding to previously published data the 11 novel mutations ( 2 in ATP1A2 and 9 in ATP1A3) we reported in this series. Collectively, up to 5\% of ATP1A2 (6/94) and 12\% of ATP1A3 (18/145) mutations can be associated with developmental and epileptic encephalopathy. Polymicrogyria can be associated with $1 \%$ of ATP1A2 and 5.5\% of ATP1A3 mutations (yellow-dashed area). Abbreviations: AHC, alternating hemiplegia of childhood; DYT, dystonia; DEE, developmental and epileptic encephalopathy (this term includes epileptic encephalopathies and developmental encephalopathies with epilepsy); FHM, familial hemiplegic migraine; PMG, polymicrogyria; RDP, rapid-onset dystonia-parkinsonism; RP, rare phenotypes (this sub-group includes CAPOS, RECA, ataxia and non-specified nervous system abnormalities).

$119 \times 42 \mathrm{~mm}(600 \times 600 \mathrm{DPI})$ 


\section{$A T P 1 A 2-$ and $A T P 1 A 3-$ associated early profound epileptic encephalopathy and polymicrogyria}

Annalisa Vetro ${ }^{1}$, Hang N. Nielsen ${ }^{2}$, Rikke Holm ${ }^{2}$, Robert F. Hevner ${ }^{3}$, Elena Parrini ${ }^{1}$, Zoe Powis, $\mathrm{MS}^{4}$, Rikke S. Møller ${ }^{5,6}$, Cristina Bellan ${ }^{7}$, Alessandro Simonati ${ }^{8}$, Gaétan Lesca ${ }^{9}$, Katherine L. Helbig $^{10}$, Elizabeth E. Palmer ${ }^{11,12}$, Davide $\mathrm{Mei}^{1}$, Elisa Ballardini ${ }^{13}$, Arie Van Haeringen ${ }^{14}$, Steffen Syrbe ${ }^{15}$, Vincenzo Leuzzi ${ }^{16}$, Giovanni Cioni ${ }^{17}$, Cynthia J. Curry ${ }^{18}$, Gregory Costain ${ }^{19}$, Margherita Santucci ${ }^{20,21}$, Karen Chong ${ }^{22}$, Grazia M. S. Mancini ${ }^{23}$, Jill Clayton-Smith ${ }^{24}$, ATP1A2/A3-collaborators ${ }^{\S}$, Stefania Bigoni ${ }^{25}$, Ingrid E. Scheffer ${ }^{26}$, William B. Dobyns ${ }^{27 *}$, Bente Vilsen ${ }^{2 *}$, and Renzo Guerrini ${ }^{1 *}$

1. Pediatric Neurology, Neurogenetics and Neurobiology Unit and Laboratories, Meyer Children's Hospital, University of Florence, Florence, Italy

2. Department of Biomedicine, Aarhus University, DK-8000, Aarhus C, Denmark

3. Department of Pathology, University of California San Diego, San Diego, CA, USA

4. Ambry Genetics, Aliso Viejo, CA, USA

5. Department of Epilepsy Genetics and Personalized Medicine Danish Epilepsy Centre, Filadelfia, Denmark

6. Department of Regional Health Services, University of Southern Denmark, Odense, Denmark

7. Department of Neonatal Intensive Care Unit, Bolognini Hospital, ASST-Bergamo Est, Seriate, Italy

8. Neurology (Child Neurology and Neuropathology), Department of Neuroscience, Biomedicine and Movement, University of Verona, Verona, Italy

9. Department of Medical Genetics, Member of the ERN EpiCARE, University Hospital of Lyon, Lyon, France

10. Division of Neurology, Children's Hospital of Philadelphia, Philadelphia, Pennsylvania

11. Centre for Clinical Genetics, Sydney Children's Hospital, Randwick, NSW, Australia

12. School of Women's and Children's Health, University of New South Wales, Randwick, NSW, Australia

13. Neonatal Intensive Care Unit, Pediatric Section, Department of Medical Sciences, Ferrara University, Ferrara, Italy 
14. Department of Clinical Genetics, Leiden University Medical Center, Leiden, Netherlands

15. Division of Pediatric Epileptology, Centre for Paediatrics and Adolescent Medicine, University Hospital Heidelberg, Heidelberg, Germany

16. Department of Human Neuroscience, Unit of Child Neurology and Psychiatry, Sapienza University, Rome, Italy

17. Department of Developmental Neuroscience, IRCCS Fondazione Stella Maris, Pisa, Italy

18. Genetic Medicine, Department of Pediatrics, University of California, San Francisco/Fresno, CA, USA

19. Division of Clinical and Metabolic Genetics, Department of Pediatrics, The Hospital for Sick Children, Toronto, Ontario, Canada

20. Child Neuropsychiatry Unit, IRCCS, Institute of Neurological Sciences, Bellaria Hospital, Bologna, Italy

21. DIBINEM, University of Bologna, Bologna, Italy

22. The Prenatal Diagnosis and Medical Genetics Program, Department of Obstetrics and Gynecology, Mount Sinai Hospital, University of Toronto, Toronto, Ontario, Canada

23. Department of Clinical Genetics, Erasmus MC University Medical Center, Rotterdam, The Netherlands

24. Manchester Centre for Genomic Medicine, University of Manchester, St Mary's Hospital, Manchester, United Kingdom

25. Medical Genetics Unit, Department of Mother and Child, Ferrara University Hospital, Ferrara, Italy

26. University of Melbourne, Austin Health and Royal Children's Hospital, Florey and Murdoch Institutes, Melbourne, Australia

27. Department of Pediatrics (Genetics), University of Minnesota, Minneapolis, USA

$\S$ The list of ATP1A2/A3-collaborators is available in the Appendix 1, with full details in the Supplementary Material

* These authors contributed equally to this work

\section{Corresponding author}

Prof. Renzo Guerrini,

Neuroscience Department, Children's Hospital Anna Meyer-University of Florence, Viale

Pieraccini 24, 50139 Florence, Italy; telephone: +39 0555662573, e-mail: r.guerrini@meyer.it 
Correspondence for Neuropathology may be addressed to:

Robert F. Hevner,

Department of Pathology, University of California San Diego, San Diego, CA, USA: rhevner@ucsd.edu

Running title: $\mathrm{Na}^{+} \mathrm{K}^{+}$-pump dysfunction and encephalopathy 


\section{Abstract}

Constitutional heterozygous mutations of $A T P 1 A 2$ and $A T P 1 A 3$, encoding for two distinct isoforms of the $\mathrm{Na}^{+}, \mathrm{K}^{+}$-ATPase (NKA) alpha-subunit, have been associated with familial hemiplegic migraine (ATP1A2), alternating hemiplegia of childhood (ATP1A2/A3), rapid-onset dystonia-parkinsonism, cerebellar ataxia-areflexia-progressive optic atrophy, and relapsing encephalopathy with cerebellar ataxia (all ATP1A3). A few reports have described single individuals with heterozygous mutations of $A T P 1 A 2 / A 3$ associated with severe childhood epilepsies. Early lethal hydrops fetalis, arthrogryposis, microcephaly, and polymicrogyria have been associated with homozygous truncating mutations in ATP1A2. We investigated the genetic causes of developmental and epileptic encephalopathies variably associated with malformations of cortical development in a large cohort and identified 22 patients with de novo or inherited heterozygous ATP1A2/A3 mutations. We characterized clinical, neuroimaging and neuropathological findings, performed in-silico and in-vitro assays of the mutations' effects on the NKA-pump function, and studied genotype-phenotype correlations. Twenty-two patients harboured 19 distinct heterozygous mutations of ATP1A2 (six patients, five mutations) and ATP1A3 (16 patients, 14 mutations, including a mosaic individual). Polymicrogyria occurred in ten $(45 \%)$ patients, showing a mainly bilateral perisylvian pattern. Most patients manifested early, often neonatal, onset seizures with a multifocal or migrating pattern. A distinctive, 'profound' phenotype, featuring polymicrogyria or progressive brain atrophy and epilepsy, resulted in early lethality in seven patients (32\%). In-silico evaluation predicted all mutations to be detrimental. We tested 14 mutations in transfected COS-1 cells and demonstrated impaired NKA-pump activity, consistent with severe loss of function. Genotype-phenotype analysis suggests a link between the most severe phenotypes and lack of COS-1 cell-survival, and also reveals a wide continuum of severity distributed across mutations that variably impair NKApump activity. We performed neuropathological analysis of the whole brain in two individuals with polymicrogyria respectively related to a heterozygous ATP1A3 mutation and a homozygous ATP1A2 mutation and found close similarities with findings suggesting a mainly neural pathogenesis, compounded by vascular and leptomeningeal abnormalities. Combining our report with all prior reports, we estimate that about 5\% of mutations in ATP1A2 and 12\% in $A T P 1 A 3$ can be associated with the severe and novel phenotypes that we describe here. Notably, a few of these mutations were associated with more than one phenotype. These findings assign novel, 'profound' and early lethal phenotypes of developmental and epileptic encephalopathies and polymicrogyria to the phenotypic spectrum associated with 
heterozygous- $A T P 1 A 2 / A 3$ mutations and indicate that severely impaired NKA pump function can disrupt brain morphogenesis.

Keywords: ATP1A2, ATP1A3, developmental and epileptic encephalopathy; polymicrogyria; $\mathrm{Na}^{+} / \mathrm{K}^{+}$-ATPase pump 


\section{INTRODUCTION}

The $\mathrm{Na}^{+} / \mathrm{K}^{+}$-ATPase (NKA) ion pump is a ubiquitous transmembrane enzyme responsible for active exchange of $\mathrm{Na}^{+}$and $\mathrm{K}^{+}$ions across the plasma membranes of higher eukaryotic cells (Kaplan, 2002). In neural tissue, this process is pivotal for maintaining the resting membrane potential, excitability, and secondary active transport that involves $\mathrm{Na}^{+} / \mathrm{H}^{+}$and $\mathrm{Na}^{+} / \mathrm{Ca}^{2+}$ exchange, $\mathrm{K}^{+} / \mathrm{Cl}^{-}$cotransport, and $\mathrm{Na}^{+}$-dependent neurotransmitter uptake (Friedrich et al., 2016). The NKA pump transports three $\mathrm{Na}^{+}$ions out of the cell and two $\mathrm{K}^{+}$ions into the cell for each ATP hydrolysed, undergoing large conformational changes between two principal conformations (E1 and E2) and their phosphorylated intermediates $\left(\mathrm{E}_{1} \mathrm{P}\right.$ and $\left.\mathrm{E}_{2} \mathrm{P}\right)$ (Kaplan, 2002; Friedrich et al., 2016; Holm et al., 2016). It is composed of a large catalytic $\alpha$ subunit and smaller $\beta$ and $\gamma$ subunits. The $\alpha$-subunit contains binding sites for three $\mathrm{Na}^{+}$ions in the $\mathrm{E} 1$ conformation or two $\mathrm{K}^{+}$ions in the $\mathrm{E} 2$ conformation, as well as a catalytic site that binds ATP and phosphate. The $\beta$-subunit is required for protein folding and targeting the $\alpha$-subunit to the plasma membrane, while both $\beta$ and $\gamma$ subunits act as fine modulators of ion affinity in different tissues (Friedrich et al., 2016).

The four known human isoforms of the $\alpha$-subunit ( $\alpha 1-4)$ encoded by four paralogous genes (ATP1A1-4) share $84-91 \%$ of the amino acid sequence and have developmental and tissue expression specificity. The isoforms differ in affinity for $\mathrm{Na}^{+}, \mathrm{K}^{+}$and ATP, and have different enzyme kinetics (Blanco, 2005). The $\alpha 2$ - and $\alpha 3$-isoforms, encoded by ATP1A2 and ATP1A3, are predominantly expressed in CNS. The expression of both isoforms is primarily neuronal during embryonic development. In the adult brain, $\alpha 3$ maintains its neuronal expression while $\alpha 2$ is primarily expressed in glial cells (McGrail et al., 1991; Moseley et al., 2003). Multiple isoforms of $\beta$ and $\gamma$ regulatory subunits also exist, with different tissue distribution and functional effects.

Constitutional heterozygous mutations of $A T P 1 A 2 / A 3$ have been associated with several autosomal dominant neurological disorders with limited overlap. These include familial hemiplegic migraine (FHM; ATP1A2 - MIM\#602481); rapid-onset dystonia-parkinsonism (RDP; ATP1A3 - MIM\#128235); alternating hemiplegia of childhood [AHC; ATP1A3-MIM \#614820 and a single family with an ATP1A2 mutation (Bassi et al., 2004; Swoboda et al., 2004)]; cerebellar ataxia-areflexia-progressive optic atrophy (CAPOS; ATP1A3 MIM\#601338); and relapsing encephalopathy with cerebellar ataxia [RECA; ATP1A3 - (Dard et al., 2015)]. Epilepsy and intellectual disability may co-occur with AHC and FHM, and severe epilepsies have been described in rare patients with ATP1A2 or ATP1A3 mutations (Paciorkowski et al., 2015; Liu et al., 2018). Early lethal hydrops fetalis, intrauterine growth 
restriction, arthrogryposis, microcephaly, polymicrogyria, and lack of respiratory drive have been associated with homozygous truncating mutations in ATP1A2 (Chatron et al., 2019; Monteiro et al., 2020).

In this study, we describe a spectrum of severe neurodevelopmental phenotypes associated with heterozygous mutations of either ATP1A2 or ATP1A3 that may include early lethality, most often occurring during status epilepticus, microcephaly and the cortical malformation polymicrogyria as well as progressive brain atrophy. Combined with prior reports of FHM, RDP, AHC, CAPOS and RECA, our data define a wide spectrum of disorders that we designate "ATP1A2/A3-opathies". We explore the detrimental effects of causative mutations on NKA pump function using several different experimental paradigms and show that the most severe phenotypes are caused by mutations that are lethal in mammalian cell culture (COS-1 cells). We also demonstrate that neuropathological features of polymicrogyria associated with heterozygous $A T P 1 A 3$ and biallelic ATP1A2 mutations are similar and support a mainly neural pathogenesis, compounded by vascular abnormalities.

\section{MATERIALS AND METHODS}

\section{Patients}

Our initial discovery cohort consisted of 2800 individuals referred to the Neuroscience and Neurogenetics Department of the Meyer Children's Hospital to investigate the genetic causes of developmental and epileptic encephalopathies (DEEs, defined according to Scheffer et al, 2017). Within this cohort, we identified de novo and inherited heterozygous mutations of ATP1A2 and ATP1A3 in nine patients (Pts 3, 6, 9, 11, 12, 14-16, and 20). Alerted by this association, we identified another 13 patients with similar features through international collaborations and by interrogating the DECIPHER database (http://decipher.sanger.ac.uk) (Pts $1,2,4,5,7,8,10,13,17,18,19,21$ and 22). We obtained written informed consent from all participants or their legal guardians according to local requirements. The study was approved by the Pediatric Ethics Committee of the Tuscany Region (grant 602531). We reviewed medical records, ECG, EEGs, and brain MRI scans. Seizure types were classified following ILAE criteria (Scheffer et al., 2017) whenever applicable and used more descriptive terms when seizure phenomenology could not be easily classified using classification terminology. We submitted all novel mutations to DECIPHER. One child with a biallelic mutation of ATP1A2 (Pt23) was described clinically in a prior report (Monteiro et al., 2020). Here we report brain autopsy findings and compare them to another child in this cohort. 
Detailed materials and methods for MRI investigations, brain neuropathology in Patients 11 and 23, genetic analysis, homology modeling and structural analysis, and functional characterization of $A T P 1 A 2$ and $A T P 1 A 3$ heterozygous mutations are reported in the Supplementary Material.

\section{RESULTS}

\section{Clinical description}

Clinical and genetic data for 22 patients (11 males/11 females) with 19 heterozygous mutations in ATP1A2 (N=6, Pts 1-6) or ATP1A3 (N=16, Pts 7-22) are summarized in Tables 1 and 2. Data for Patient 23, carrying a biallelic ATP1A2 mutation and not considered in the clinical study, is also included in the Tables.

Epilepsy was reported in 21/22 individuals, with seizure onset during the neonatal period in 11 individuals and during infancy or childhood in the remaining nine. Refractory status epilepticus had occurred in 10 patients, in five of whom as the presenting type of seizure in the neonatal period (Pts 3, 4, 7, 11, and 13). Most had multiple seizure types, with multifocal $(\mathrm{N}=9)$ and focal seizures $(\mathrm{N}=7)$ being most frequent. The multifocal seizure pattern observed in these patients corresponded to the epilepsy of infancy with migrating focal seizures (EIMFS) syndrome (Burgess et al., 2019). Recurrent, prolonged, seizure-related apneic episodes, accompanied by intense cyanosis, were a prominent feature in eight patients ( $\mathrm{Pts} 3,7,11,12$, $16,17,20,21)$. In two additional neonates, apneic episodes were attributed to central apnea (Pts 14 and 18). A burst suppression EEG pattern was documented in two children (Pts 3 and 11). Multiple antiseizure medications were used both in the acute treatment of status epilepticus/prolonged seizures and as chronic treatments, with no evidence of any drug being more effective than any other or causing seizure worsening.

Survival was often short, as eight children died due to complications of status epilepticus (Pts $3,4,7,10,11,17,19)$, or intercurrent respiratory infections (Pt 18); seven of these children had died between ten days and 30 months of life (Pts 3, 4, 7, 11, 17-19) and one at 14 years (Pt 10). Overall, four children's demise happened within the first two months of life (Pts 4, 7, 17 and 19), too early to detect progressive worsening, which was instead apparent in the remaining four who died at a later age. 
ECG data, available for 19 children (ATP1A2: Pts 2-4, 6; ATP1A3: Pts 7-14, 16-22) were unremarkable in all, except Patient 13 in whom a short QT interval with unusual ST segment was reported, which had remained asymptomatic at age 3 years (Table 1).

Severe congenital or postnatal (usually by age two years) microcephaly was observed in 10/22 patients. Global developmental delay and intellectual disability was documented in 18/22 patients, including all who survived long enough to be assessed. The disability was rated as severe to profound in 14 , moderate in three, and mild in 1/18 patients. Severe axial hypotonia was reported in 14 individuals including three with dyskinetic quadriparesis (Pts 2, 10, 18). Attacks of AH were observed in only two children (Pts16 and 20), both having polymicrogyria. Additional clinical findings in this cohort included Pierre-Robin sequence (micrognathia with cleft palate, Pt11), optic nerve atrophy (Pt10), and hypogenetic lung syndrome (Pt14).

\section{Brain imaging}

All 22 patients with ATP1A2/A3 heterozygous mutations had at least one brain MRI scan and six were scanned more than once at greater than 1-year intervals. The main finding, observed in ten patients (45\%), was polymicrogyria (nine ATP1A3 mutations: Pts 7, 11, 13-16, 17, 19 and 20; one ATP1A2 mutation: Pt6), which was perisylvian predominant, bilateral in nine patients and unilateral in one (Pt15), inconstantly involving other areas (Fig. 1). Perisylvian involvement was accompanied in all by severe disruption of the sulcal pattern, with a verticallyoriented and extended Sylvian fissure. Associated findings included microcephaly in $7 / 10$ and thick corpus callosum in 4/10 patients (Table 1, Fig. 1). Two subjects with polymicrogyria had repeated brain MRI studies that showed no signs of progressive changes.

Severe and progressive brain atrophy was instead documented in two patients, without polymicrogyria (Pts 2 and 8, Supplementary Fig. 1). In the ten remaining patients, brain MRI was normal or showed mild atrophic changes, and remained so in the two who were scanned twice (Pts 9 and 10).

\section{Neuropathological brain examination}

In Patient 11, with a heterozygous ATP1A3 mutation (A3-K764del), and in Patient 23, with a homozygous ATP1A2 frameshift mutation (A2-R279G*4), we performed post-mortem neuropathological examination and compared findings (Fig. 2 and 3).

The brain of Patient 11 appeared small for the age of four postnatal months (brain weight not available). The surface gyri were abnormal, mostly small and irregular, but with apparent 
pachygyria of pericentral cortex bilaterally, and bilateral widening of the central sulcus (Fig. 2A-C). In slices, the corpus callosum was present but small at the genu and was truncated with lack of midline crossing at the level of the posterior body and splenium, thus forming Probst bundles (Fig. 2G). The regions of surface pachygyria showed abnormally thick cortical gray matter (Fig. 2D,E). Hemispheric white matter was also reduced. The basal ganglia and thalami appeared relatively spared, with almost normal size. The cerebellar folia showed no obvious defects. The cerebellar dentate nuclei and the inferior olives had simplified morphology, and no arcuate nuclei were detected. Histology revealed that the macroscopically pachygyric cortex was thick, disorganized, and partially covered by rinds of leptomeningeal glioneuronal heterotopia (Fig. 2F,G). Histology also demonstrated that the macroscopic appearance of pachygyria actually corresponded to disorganized layers with some features of classic fourlayered polymicrogyria (arrowheads, Fig. 2F,G); the pachygyric appearance was conferred in part by the rind of leptomeningeal heterotopia. Some areas exhibited more definitive features of polymicrogyria (arrows, Fig. 2F,G), while other areas showed essentially normal cortical lamination. Overall, these findings indicated a variable malformation of cortical development affecting central regions more severely that was also associated with malformations of brainstem and cerebellar nuclei.

The brain of Patient 23 was very small for the age of 33 gestational weeks $(91.5 \mathrm{~g}$; expected 220 g). Surface gyri were difficult to assess due to adherent meninges with subarachnoid hemorrhage (agonal) and congested blood vessels (Fig. 3A,B). Midline section revealed that the corpus callosum was small but present, including the splenium (arrow Fig. 3B). The cerebellum was moderately hypoplastic, with reduced foliation (Fig. 3C). Coronal slices through the hemispheres revealed severe hypoplasia of gray and white structures, as well as moderate enlargement of the posterior lateral ventricles (i.e. colpocephaly: Fig. 3D-F). The cortical gyri were diffusely small with extensive folding, suggestive of polymicrogyria, except for the hippocampus, which appeared relatively normal bilaterally (arrows, Fig. 3E). Histology confirmed diffuse polymicrogyria throughout the cortex except in the hippocampus, which displayed relatively normal lamination (Fig. 3G,H). Leptomeningeal glioneuronal heterotopia were widespread over the external surface of the hemispheres (Fig. 3I). Histology also revealed abundant focal calcifications (or mineralization) involving cerebral gray and white matter, leptomeninges and blood vessels (arrows, Fig. 3G-L). Some blood vessels had abnormally thick walls (intimal hyperplasia) as well as mineralizations (Fig. 3L). Also, the cerebellar deep nuclei were dysplastic, and the pyramidal tracts were severely hypoplastic (not shown). 


\section{Genetic findings}

We identified 19 heterozygous mutations of ATP1A2 $(\mathrm{N}=5)$ or ATP1A3 $(\mathrm{N}=14$; Table 1) including 15 missense substitutions, three in-frame deletions, and one insertion. All 19 mutations affected highly conserved residues among both orthologs and paralogs of ATP1A2 and ATP1A3 (Table 1, Supplementary Fig. 2) and were interpreted as pathogenic based on both in silico analysis using sequence data and the functional studies described below. Two mutations (A2-G366A and A3-S361P) were recurrent, occurring in two individuals each. The mutations occurred de novo in 20/22 patients and were inherited in two individuals from one family (Pts 14-15: A3-D887Y). The latter was inherited across three generations from a reportedly healthy man to his daughter (Pt15) and grandson (Pt14). None of the mutations were present in either GnomAD or our internal dataset.

Analysis of the variant allele frequency (VAF) showed that 21/22 mutations were constitutional and one mosaic with VAF $26.2 \%$. Eleven heterozygous mutations were novel (ATP1A2 N=2; ATP1A3 N=9; Table 1). Of the eight mutations previously reported, three were associated with FHM (Pt6: A2-R908Q) or AHC (Pt10: A3-D609Y; Pt13: A3-D801N) (De Vries et al., 2007; Heinzen et al., 2012; Rosewich et al., 2012; Marzin et al., 2018). The mosaic mutations found in our series (Pt21: A3-F857del) was reported as a constitutional mutation with no clinical details in one individual (Retterer et al., 2016). Four additional mutations found in Patients 1 , 3, 16 and 22 reported here had been mentioned in previous series within which these patients had been included (Panagiotakaki et al., 2015; Parrini et al., 2017; Ueda et al., 2018; Burgess et al., 2019). No additional potentially pathogenic mutations were identified in the 20 patients with available WES/WGS data and in the two examined by TGP (Pts 2 and 5).

\section{Homology modeling and structural analysis}

Of the 19 mutations in our cohort, eight ATP1A3 mutations, but only two ATP1A2 mutations, affected the transmembrane helices M3 (A3-L292R, A2-I293M), M4 (A3-G316V, A2-C341F), M5 (A3-K764del, A3-P775R), M6 (A3-D801N), M7 (A3-F857del), M8 (A3-L924P), and M10 (A3-D992dup) (Fig. 4 and Supplementary Fig. 3). The predicted structural impacts of all mutations in our cohort are detailed in Supplementary Table 1 and illustrated in Supplementary Fig. 4-10. A3-D801N has previously been shown to impair $\mathrm{Na}^{+}$and $\mathrm{K}^{+}$transport as $\mathrm{D} 801$ is known to directly bind $\mathrm{Na}^{+}$and $\mathrm{K}^{+}$at both sites I and II (Weigand et al., 2014; Li et al., 2015; Holm et al., 2016). A3-L924P is predicted to break the M8 helix, thereby indirectly disturbing the interaction of the juxtaposed D923 with $\mathrm{Na}^{+}$at site III (Arystarkhova et al., 2019). The 
mutations A3-L292R, A2-I293M, A3-G316V, A2-C341F, A3-K764del, A3-P775R, A3L924P, and A3-D992dup found in transmembrane helices and A3-P972del in the L8-9 loop connecting the transmembrane helices M8 and M9 are predicted to affect the ion binding sites indirectly. Four mutations affected the P domain, which contains the phosphorylation site (A2G366A, A2-R593Q, A3-S361P, and A3-D609Y). A3-D609Y replaces the aspartate of the conserved TGD motif critical for ATP binding (Holm et al., 2016). The remaining four mutations (A2-R908Q, A3-D887Y, A3-L888P, A3-G893W) affected the L7-8 extracellular loop between transmembrane helices M7 and M8, which interacts with the extracellular domain of the $\beta$-subunit. Hence, these mutations are predicted to interfere with the $\alpha$ - $\beta$ interaction crucial to expression in the plasma membrane. These four mutations were all associated with polymicrogyria. A3-G893W introduces a bulky tryptophan corresponding to the glycine of the SYGQ motif at the interface with the $\beta$-subunit. A3-F857del is likely to affect the interaction between M7 and the transmembrane helix of the $\beta$-subunit. No mutations involved the Nterminal third of the protein including M1-M2 and the A domain.

\section{Functional characterization of $A T P 1 A 2-A 3$ heterozygous mutations}

The primary function of ATP driven NKA pumps is to rapidly exchange three intracellular $\mathrm{Na}^{+}$ ions for two extracellular $\mathrm{K}^{+}$ions. In the presence of ATP and intracellular $\mathrm{Na}^{+}$, the ATP-NKA pump in its E1 state forms a phosphorylated intermediate (E1P) that changes configuration from the E1P to E2P state, exposing $\mathrm{Na}^{+}$to the extracellular space. In the presence of extracellular $\mathrm{K}^{+}$, three $\mathrm{Na}^{+}$are released and two $\mathrm{K}^{+}$bound (Fig. 5D). The phosphorylated intermediate (E2P) is hydrolyzed to the E2 state which equilibrates to the E1 state in the presence of intracellular ATP, delivering two $\mathrm{K}^{+}$to the intracellular space.

Among the Cos- 1 cell lines transfected with the 14 mutations we tested, only three ATP1A2 and two $A T P 1 A 3$ mutants retained the $5-10 \%$ wild type NKA pump activity required for cell survival under ouabain selection (Holm et al., 2016). The remaining two ATP1A2 and seven ATP1A3 mutants could not support cell growth in stable transfections, indicating that they lacked NKA pump activity or were not expressed in the plasma membrane (Table 2 and Supplementary Table 1). Hence, we analysed these mutants by transient transfection into COS1 cells using siRNA to knock down the endogenous NKA pump, as described previously (Nielsen et al., 2019).

Among the five stably expressed mutants retaining transport activity (cell survival), only A2I293M and A2-R593Q showed phosphorylation levels $>50 \%$ wild-type, whereas levels of A2R908Q, A3-P972del, and A3-D887Y were 26-35\% wild-type (Fig. 5A, Table 2). However, the 
turnover rate (rate of $\mathrm{Pi}$ liberation per active site in the presence of both $\mathrm{Na}^{+}$and $\mathrm{K}^{+}$) was markedly reduced for A2-I293M (27\% wild-type) and A2-R593Q (42\% wild-type) (Fig. 5B, Table 2). Hence, the NKA pump activity calculated by multiplying the phosphorylation level by the turnover rate was markedly reduced for all five mutants (17-36\% wild type), thus accounting for the pathogenic effects of all five stably expressed mutations (Table 2).

Among the nine remaining mutants that could only be transiently expressed because of lack of NKA pump activity (Fig. 5C), the phosphorylation levels of A2-G366A (61\%), A3-L292R (61\%), and A3-G316V (70\%) indicated that they were expressed in the plasma membrane and retained the ability to bind $\mathrm{Na}^{+}$and ATP, despite their inability to undergo the complete transport cycle.

To explore the $\mathrm{Na}^{+}$- and $\mathrm{K}^{+}$-binding properties of the mutants, we took advantage of the $\mathrm{Na}^{+}$and $\mathrm{K}^{+}$-dependence of NKA phosphorylation and pump activity, which could be studied for the stably as well as the transiently expressed mutants showing robust phosphorylation. The effects of mutations on the E1-E2 and E1P-E2P conformational changes (cf. reaction cycle in Fig. 5D) are summarized in Fig. 6A,B, Supplementary Fig. 11, and 12, with full text presentation in the Supplementary Materials. The very low or undetectable phosphorylation (expression) levels in the remaining six mutants (A2-C341F, A3-S361P, A3-K764del, A3-P775R, A3-L888P, and A3-P992dup), prevented further study of their phosphorylation properties (Fig. 5C).

In brief, the mutations in the transmembrane helices for which the ion binding properties could be examined (M3: A2-I293M and A3-L292R, M4: A3-G316V, Supplementary Fig. 4 and 9), profoundly interfered with $\mathrm{Na}^{+}$and/or $\mathrm{K}^{+}$binding in various ways. $\mathrm{Na}^{+}$binding was also disturbed by one of the mutations in the P-domain (A2-G366A, Supplementary Fig. 6) and the mutation in the L9-10 loop (A3-P972del, Supplementary Fig. 10). In addition, A2-G366A disturbed $\mathrm{K}^{+}$binding, as the E2P phosphoenzyme intermediate of this mutant was completely insensitive to $\mathrm{K}^{+}$. The M3 mutant (A2-I293M, Supplementary Fig. 4) had a strong conformational shift in favor of E1/E1P explaining the reduced turnover rate. A similar, but less strong conformational shift was seen for the P-domain mutation near the ATP binding site (A2-R593Q, Supplementary Fig. 7). The remaining mutations in transmembrane helices (A2C341F, A3-K764del, A3-P775R, and A3-D992dup, Supplementary Fig. 5, 9, and 10) resulted in low or undetectable phosphorylation levels, either due to inability to react with $\mathrm{Na}^{+}$and ATP to form a phosphoenzyme, or lack of expression of the mutant in the plasma membrane. The same was the case for the P-domain mutation (A3-S361P) located five residues from the phosphorylated aspartate (Supplementary Fig. 6). Mutations in the L7-8 loop, interacting with the $\beta$-subunit crucial to expression in the plasma membrane (Supplementary Fig. 8), also 
reduced the phosphorylation level, either partially (A3-D887Y and A2-R908Q) or completely (A3-L888P), most likely due to reduced expression.

In synthesis, in every patient whose mutations were tested at least one of the assays used showed severe impairment, as summarized in Table 2. The 11 mutations not supporting cell growth in culture were associated with the most severe clinical phenotype, while the five mutations supporting cell growth were associated with more variable clinical phenotypes.

\section{DISCUSSION}

Since the first reports of ATP1A2 and ATP1A3 mutations in 2003-2004 (De Fusco et al., 2003; De Carvalho Aguiar et al., 2004) more than 200 mutations (N=92 ATP1A2, $\mathrm{N}=136$ ATP1A3) have been identified [Human Gene Mutation Database (HGMD) Professional 2018.3, https://portal.biobase-international.com, last accessed June 2020]. Autosomal dominant disorders associated with ATP1A3 mutations have been conceptualized as a phenotypic continuum that includes RDP, AHC, CAPOS, RECA, and DEE (Rosewich et al., 2014; Dard et al., 2015; Paciorkowski et al., 2015). ATP1A2 mutations have been associated with FHM and, in a single family, AHC (Bassi et al., 2004; Swoboda et al., 2004), defining a much narrower and less severe phenotype than ATP1A3 (Fig. 7).

Here we describe 22 individuals with heterozygous ATP1A2 or ATP1A3 mutations associated with developmental encephalopathies manifesting most often as early-onset DEE, and associated with frequent early lethality (in 32\%) and polymicrogyria (in 45\%). Clinical and functional findings assign these novel, 'profound' phenotypes to the existing spectrum of disorders associated with heterozygous $A T P 1 A 2 / A 3$ mutations and demonstrate that severely impaired $\mathrm{Na}^{+}, \mathrm{K}^{+}$-pump function can disrupt brain morphogenesis.

Combining our report of 19 mutations (11 novel) with all prior reports, we estimate that about $5 \%$ of mutations in ATP1A2 and 12\% in ATP1A3 are associated with the severe and novel phenotypes that we describe in this series. Notably, a few of these mutations were associated with more than one phenotype (Fig. 7).

\section{Epilepsy}

Epilepsy occurs in about 50\% of patients with AHC and 15-30\% of those with FHM (Deprez et al., 2008; Heinzen et al., 2012; Rosewich et al., 2012; Prontera et al., 2018). In our cohort, which was assembled based on clinical features of developmental encephalopathy, epilepsy occurred in 21 of 22 patients $(95 \%)$, with neonatal or early infantile onset in most and episodes 
of status epilepticus in 10 (45\%). Status epilepticus has been reported to occur in up to $40 \%$ of patients with ATP1A3-AHC (Panagiotakaki et al., 2015) but is a less frequent feature in ATP1A2-FHM (Deprez et al., 2008; Prontera et al., 2018). The occurrence of multiple seizure types, especially multifocal seizures manifested as EIMFS, in most patients indicates widespread epileptogenesis and brain dysfunction. Patients 3 and 4 exhibited a remarkably severe phenotype among those with $A T P 1 A 2$ mutations reported so far, manifesting neonatal onset super-refractory status epilepticus featuring prolonged apneic episodes. Both patients died within the first year of life without achieving developmental or motor skills. Prolonged seizurerelated apneic episodes were a recurrent feature in five additional individuals, all exhibiting ATP1A3 mutations. In two further patients with ATP1A3 mutations, similar apneic episodes lacked any electrographic correlate and were classified as central apnea. In these patients, structure of the brainstem was intact, suggesting a disturbance of respiratory system regulation. Respiratory disturbances have been described in ATP1A3-AHC (Rosewich et al., 2014). Biallelic ATP1A2 mutations have also been associated with respiratory failure due to absence of spontaneous respiratory effort (Chatron et al., 2019; Monteiro et al., 2020) resembling knockout Atpla 2 or Atpla3 mice, whose defective respiratory rhythm generation was attributed to failed $\mathrm{Cl}^{-}$clearance through the $\mathrm{K}-\mathrm{Cl}$ transporter $(\mathrm{KCC} 2)$ in brainstem postsynaptic neurons (Ikeda et al., 2003, 2017). The KCC2 transporter relies on the $\mathrm{K}^{+}$gradient created by the NKA to pump $\mathrm{Cl}^{-}$out of the cells. The same pathophysiological mechanism might cause the nonepileptic episodic respiratory disturbances in Patients 14 and 18.

Patients with ATP1A3-related disease are at increased risk of exhibiting dynamic ECG abnormalities, a finding substantiated by the multiple cardiac rhythm abnormalities observed the in murine Atpla3 model (Balestrini et al., 2020). However, while sudden seizure-related cardiac death has been induced in murine Atpla3-related disease, premature mortality remained limited to $2 \%$ in the international AHC-ATP1A3 registry cohort over a 20 years period (Rosewich et al., 2017), mainly as a consequence of accidental events or respiratory complications of status epilepticus. In our series, a short QT interval, an ECG abnormality which is potentially predisposing to life threatening arrhythmia, was present in one patient ( $\mathrm{Pt}$ 13) and was the only abnormal finding observed among 19 patients whose ECGs were available for inspection. This patient's mutation, the A3-D801N, a highly recurrent AH mutation, was also present in one patient with AH and short QT reported by Balestrini et al (Balestrini et al., 2020). The six patients with ATP1A3 mutations who died prematurely in our series had normal ECGs. These patients died either as a consequence of an intercurrent respiratory infection or during status epilepticus after progressive worsening of their conditions. These circumstances 
of death were not consistent with a sudden cardiac event but it cannot be excluded that a compromised cardiac function might have contributed to these patients' vulnerability.

\section{Brain MRI abnormalities and neuropathological findings}

Polymicrogyria, which we observed with consistent features in ten patients $(45 \%)$, is a novel phenotype for heterozygous $A T P 1 A 2$ and $A T P 1 A 3$ mutations. It is possible that previously reported patients may not have had adequate MRI imaging and thus subtle alterations of the cortical folding might have been overlooked in older studies. In addition, phenotype-guided genetic testing, largely applied in the past, might have prevented novel malformation phenotypes from being found.

In one qualitative MRI study, brain and cerebellar atrophy were reported in up to $50 \%$ of patients with ATP1A3-AHC (Sasaki et al., 2017). Microcephaly with rapidly progressive brain atrophy was described in a single patient with ATP1A3-related DEE (Paciorkowski et al., 2015). Two quantitative MRI studies in small series confirmed reduced total brain volume, with prevalent white matter involvement (Severino et al., 2020) and mild, possibly age related, cerebellar atrophy (Ghusayni et al., 2020).

Polymicrogyria is etiologically heterogeneous and variable in topography (Guerrini and Dobyns, 2014). In this series, the malformation was either limited to the perisylvian cortex or more diffuse but perisylvian predominant. Its characteristics shared similarities with those observed with mutations in several developmental genes (the microcephaly gene WDR62, multiple tubulin genes, the PIK3CA-AKT pathway associated PIK3R2 gene) but recently also associated with the ion channel genes SCN3A (Smith et al., 2018; Zaman et al., 2020) and, in more severe forms, GRIN1 and GRIN2B (Platzer et al., 2017; Fry et al., 2018).

Mutations of $S C N 3 A$ were initially associated with non-malformation epilepsy and DEE (MIM \#617935; MIM \#617938), and more recently with polymicrogyria (Smith et al., 2018; Zaman et al., 2020). However, mutations associated with polymicrogyria proved to be gain-of-function resulting in increased non-inactivating persistent $\mathrm{Na}^{+}$currents $\left(\mathrm{I}-\mathrm{Na}_{\mathrm{P}}\right)$. Changes in the sodium flux in glia and neurons may reverse $\mathrm{Na}^{+} / \mathrm{Ca}^{2+}$ exchangers triggering calcium waves (Smith et al., 2018; Zaman et al., 2020).

In GRIN1-associated polymicrogyria, gain-of-function mutations causing hyperactivation of NMDA receptors increase intracellular $\mathrm{Ca}^{2+}$, which was predicted to induce polymicrogyria either by causing increased cell death during foetal brain development (excitotoxicity) or by activating signalling pathways such as PI3K-AKT (Fry et al., 2018). A similar mechanism 
would be at play with GRIN2B mutations (Smith and Walsh, 2020). Altered NMDA receptormediated calcium mobilization disturbs neuronal migration in a mouse model of Zellweger syndrome, a rare metabolic disorder featuring a polymicrogyria-like cortical malformation (Gressens et al., 2000). The altered functioning of the NKA pump provides further evidence that changes in ion homeostasis during embryogenesis may disrupt cortical development (Smith and Walsh, 2020).

One child from a large family segregating FHM (A2-R348P) was reported to have lissencephaly with microcephaly and retinal dysplasia, likely an unrelated phenotype (Pelzer et al., 2017). One of the mutations found in our series has been reported in about $40 \%$ of individuals with AHC (Pt13: A3-D801N) with no brain malformation reported (Rosewich et al., 2012). Different phenotypes associated to the same mutation are not new to ATP1A3. The D923N mutation is recurrent in RDP but has also been observed in one family with four affected individuals with AH (Roubergue et al., 2013). These authors postulated that these clinical syndromes represent the different expressions of the same disorder and that the specific ATP1A3 mutation is not the only determinant of clinical expression, implying that genetic, epigenetic, and environmental factors may all influence clinical expression of ATP1A3 related disease. The A2-R908Q mutation was associated with early onset epileptic encephalopathy in one patient in our series and with familial hemiplegic migraine in multiple unrelated individuals in the literature (De Vries et al., 2007; Roth et al., 2014; Blicher et al., 2016), demonstrating that phenotypic heterogeneity is not limited to ATPIA3 mutations.

We observed variable expressivity for polymicrogyria with one mutation in our series, in which two unrelated individuals carrying the same ATP1A3 mutation (Pts 9 and 20: A3-S361P) had early onset DEE and microcephaly, associated with polymicrogyria and alternating hemiplegia only in one (Pt20). We also observed incomplete penetrance and variable expressivity within a single family in which the proband (Pt14: A3-D887Y) had early onset DEE and bilateral polymicrogyria, his mother (Pt15) had migraine, mild intellectual disability and unilateral polymicrogyria, and the maternal grandfather had no reported symptoms and a normal brain MRI despite carrying a germline mutation. Incomplete penetrance and variable expressivity have also been observed with $S C N 3 A$-associated polymicrogyria (Smith et al., 2018). Similarly, GRIN1 mutations even within the same functional domain can result in DEE with normal MRI or polymicrogyria (Fry et al., 2018).

Neuropathological study of the whole brain in Patients 11 (A3-K764del) and 23 (homozygous A2-R279Gfs*4), displayed substantially similar findings. The most obvious similarities were 
small size of the hemispheres with widespread polymicrogyria covered by extensive leptomeningeal glioneuronal heterotopia, confirmed histologically in both brains. Also, the white matter was atrophic and the corpus callosum small, suggesting that cortical axons were deficient. The most striking difference between the two brains were the abundant mineralizations seen in the brain and blood vessels of Patient 23, which were not observed in Patient 11. Extensive vascular and parenchymal mineralization was also reported in previous clinical and pathological studies of patients with polymicrogyria and biallelic ATP1A2 mutations (Chatron et al., 2019). These findings suggest that ATP1A2 may be important for development and function of both neural and vascular tissue in the brain.

The known expression patterns of $A T P 1 A 2$ and $A T P 1 A 3$ support the microcephaly and polymicrogyria seen in our series (mouse: http://www.informatics.jax.org; human: http://www.hdbr.org/expression). In developing mouse brain, Atpla2 is highly expressed in neural progenitors and meninges, then persists after birth in glial cells (McGrail et al., 1991; Herrera et al., 1994; Moseley et al., 2003). Atpla3 is also expressed in neural progenitors but not in meninges and persists after birth in postnatal neurons (McGrail et al., 1991; Herrera et $a l .$, 1994). Thus, both genes could be implicated in direct injury to radial glia and migrating neurons. In sum, the expression patterns of $A T P 1 A 2 / A 3$ in developing brain combined with observations in two brains from our series support a mainly neural (radial glia, progenitors, migrating neurons) pathogenesis that may be compounded by meningeal and vascular abnormalities. No obvious brain anatomical defects have been reported in ATP1A2/A3 mouse models (Ikeda et al., 2003, 2017). However, mouse models are not particularly suitable to unveil abnormal cortical folding and defective cortical lamination might have remained unnoticed as no specific studies seem to have investigated it.

Different studies have suggested a direct link between the $\mathrm{Na}+, \mathrm{K}+$-ATPase and signal transduction pathways involving, among others, the Src family kinases, and PI3K, which has been implicated in a spectrum of brain malformations. According to these studies, the transmembrane NKA pump would act as a scaffold, tethering signalling proteins together into distinct cellular compartments, thus contributing to the modulation of signal transduction within the cell (Reinhard et al., 2013).

\section{Structural/functional considerations}


A clustering of AHC-associated ATP1A3 mutations in transmembrane domains 2-9 has been previously reported (Rosewich et al., 2014; Holm et al., 2016). Conversely, FHM-associated ATP1A2 mutations and RDP-associated ATP1A3 mutations are scattered throughout the protein (Rosewich et al., 2014; Holm et al., 2016).

In our cohort we observed an enrichment of ATP1A3 mutations in transmembrane domains M3M10 clustered around the ion binding sites (8/14; Supplementary Fig. 3), as previously noted for AHC-associated mutations, suggesting that the most severe ATP1A3-related phenotypes may be associated with this location (Sweadner et al., 2019). There was no clustering distribution of ATP1A2 mutations in our series, although the A2-G366A mutation, affecting the P domain, was recurrent in two unrelated patients with super-refractory status epilepticus who died prematurely (Pts 3,4).

Most of the mutations found in our cohort result in severe functional defects based on both disruption of protein structure and biochemical analysis. Many of the mutations replace or insert bulky and/or charged residues such as aromatic amino acids, proline or arginine with smaller or less charged residues, while a few simply delete or duplicate a residue. All 14 of the mutations we tested caused reduced NKA pump activity in the COS-1 cellular model. In nine mutants the NKA pump activity was too low for survival of the transfected COS-1 cells, substantiating the 'profound' phenotypes featuring early death, DEE, progressive brain atrophy and polymicrogyria observed in most patients carrying these mutations (Table 2). The very low pump activity results from severely reduced expression level, inability to form a phosphoenzyme or, for the phosphorylatable mutants A3-L292R, A3-G316V and A2-G366A, a severe reduction in $\mathrm{Na}^{+}$and/or $\mathrm{K}^{+}$affinity. The remaining five mutations caused reduced (17$36 \%$ ) activity that was sufficient for cell growth, attributed to either reduced expression level in the plasma membrane (A2-R908Q, A3-P972del, and A3-D887Y), or an E1P shift of the E1PE2P conformational equilibrium (A2-I293M, A2-R593Q).

Based on the impaired NKA pump activity observed in the COS-1 cells, where only the mutant NKA is expressed, we classify the mutations in our cohort as loss-of-function, including those mutants for which the impaired pump activity is due to reduced plasma membrane targeting, as previously indicated for A2-R908Q (Tavraz et al., 2009).

Dominant negative effects, which have previously been suggested for A3-D801N (Pt3), A3E815K and A3-G947R (Li et al., 2015) may also be contributing to the severe phenotype in our series. A number of factors argue against haploinsufficiency as the main pathogenetic mechanism, including absence of phenotypic manifestations in carrier parents, similarities of the neuropathological polymicrogyria phenotype with that observed with biallelic mutations 
[Pt23, (Chatron et al., 2019)] and the nonmalformative phenotypes observed in individuals with large deletions involving ATP1A2/A3 (DECIPHER, https://decipher.sanger.ac.uk/; ClinVar, https://www.ncbi.nlm.nih.gov/clinvar/). Misfolding of the mutant $\alpha$-subunit and allele competition for the $\beta$-subunit might also contribute to deleteriousness of ATP1A3 mutations and their phenotypic heterogeneity (Arystarkhova et al., 2019).

Sweadner et al (Sweadner et al., 2019) suggested that genes that only diverged slightly after past duplications, such as ATP1A2 and ATP1A3, would be expected to harbor similar pathogenic mutations, but observed that only relatively few mutations affected the corresponding amino acids in these two genes. Our study provides additional examples of mutations affecting homologous residues in ATP1A2 and ATP1A3 (Supplementary Table 1). The ATP1A2 glycine 366 (A2-G366), which is mutated in Patients 3 and 4 (A2-G366A) with early lethal phenotypes, corresponds to the G358 residue in ATP1A3 mutated in a newborn with seizures and progressive brain atrophy who died prematurely (G358V) (Paciorkowski et al., 2015). Different de novo substitutions of A2-G366 have been reported in one patient with refractory epilepsy (A2-G366V) (Liu et al., 2018) and in another with a nonspecific neurodevelopmental disorder (A2-G366C) (McRae et al., 2017). A2-G366, or its equivalent in ATP1A3, is located in the P-domain at the junction between the P1-helix and the P1- $\beta$-strand leading directly to the phosphorylated active site aspartate of the DKTGT motif (Supplementary Fig. 6). The glycine is important for the direction of this $\beta$ strand. In COS-1 cells transfected with the A3-G366A, the NKA showed a 5-fold reduction in $\mathrm{Na}^{+}$affinity and a highly reduced apparent $\mathrm{K}^{+}$affinity too, with lack of transport activity due to lack of $\mathrm{K}^{+}$-induced dephosphorylation of E2P.

\section{Genotype-phenotype analysis}

The phenotypes observed in our cohort were predominately severe, with short survival (death at 0-3 years) observed in 7/22 (32\%), severe congenital or postnatal microcephaly in 10/22 (45\%), polymicrogyria in 10/22 (45\%), and severe or profound early developmental delay or intellectual disability in 14/18 (78\%) individuals. We tested 14 mutations from 17 patients and next separated our patient cohort into two groups based on COS-1 cell survival in ouabain selection pressure (Table 2). Group one included 11 patients harbouring 9 different mutations, all resulting in lack of cell survival; we observed early mortality in 5/11 (45\%), severe microcephaly in 6/11 (55\%), polymicrogyria in 5/11 (45\%) and severe or profound intellectual disability in 8/9 (89\%) patients. Group 2 included six patients whose mutations were associated with cell survival; we found early mortality and severe microcephaly in only 1/6 (17\%) each, 
polymicrogyria in 3/6 (50\%) and severe or profound intellectual disability in $2 / 6(33 \%)$ patients. A comparison of the two groups suggests a trend toward more severe phenotype in Group 1, but the differences were not statistically significant due to small numbers (Fisher's Exact Test). Further, all of the phenotypes in both groups were relatively severe, which fits with our finding of severe functional defects in various experimental paradigms for all mutations (Table 2).

For example, seven children (five from Group 1, one from Group 2, and a further patient with the untested A3-G893W) died at 0-3 years with profound encephalopathies classified as DEE. In Group 1, two individuals without polymicrogyria carried the severe A2-G366A mutation, while the remaining three all had polymicrogyria with mutations affecting the transmembrane helices (A3-L292R, A3-K764del, and A3-D992dup) and causing severe impairment of NKA pump activity. A3-L292R caused severe reduction of $\mathrm{Na}^{+}$and $\mathrm{K}^{+}$affinity, compatible with the location of the bulky, positively charged arginine substituent in position to repel $\mathrm{Na}+$ and $\mathrm{K}^{+}$at their binding sites (Supplementary Fig. 9). For A3-K764del and A3-D992dup, phosphorylation and expression were very low or undetectable, most likely caused by misfolding and/or disruption of $\mathrm{Na}^{+}$binding, as these residues are involved in an intricate hydrogen bonding network that is essential for positioning of the transmembrane helices and C-terminus forming the fold for $\mathrm{Na}+$ binding at site III (Supplementary Fig. 10).

The remaining 15 subjects survived infancy although one later died at 14 years. We tested ten mutations from this group, including five (A2-C341F, A3-G316V, A3-S361P, A3-P775R, A3L888P) that resulted in loss of NKA pump activity as indicated by lack of COS- 1 cell survival. The six children harbouring these five mutations all had severe phenotypes that included intractable epilepsy and severe intellectual disability, variably associated with microcephaly, polymicrogyria and alternating hemiplegia (A3-L888P and A3-S361P), severe hypotonia, and/or progressive brain atrophy (A2-C341F, A3-G316V, A3-S361P). The two patients with alternating hemiplegia in this group (Pts 16 and 20) were the only ones to manifest this disorder in the entire series. However, recognition of attacks may have been hampered by severe hypotonia and frequent seizures in the other children. In Pt8:A3-G316V, the low pump activity was associated with low $\mathrm{K}^{+}$affinity, compatible with insertion of a bulky valine close to the ion binding sites (Supplementary Fig. 9). Phosphorylation and expression were very low or undetectable for A2-C341F, A3-S361P, A3-P775R, and A3-L888P. For A3-S361P, the lack of phosphorylation most likely results from redirection of the $\beta$-strand leading to the phosphorylated aspartate by insertion of a proline five residues before the aspartate (Supplementary Fig. 6). For A2-C341F and A3-P775R, the inserted bulky side chains appear 
to disturb ion binding and helix packing in the membrane, especially when charged arginine is involved (Supplementary Fig. 5 and 9). A3-L888P inserts a proline redirecting the L7-8 loop, predicted to disrupt the interaction between the $\alpha$ - and $\beta$-subunits, which should prevent expression in the plasma membrane (Supplementary Fig. 8).

The remaining five mutations resulted in partially conserved NKA pump activity allowing long term COS-1 cell survival. In the six subjects harbouring these mutations, the phenotypes were of variable severity. Three patients (Pt6: A2-R908Q; Pt14-15: A3-D887Y; Pt18: A3-P972del) had severe phenotypes that overlap with those associated with COS-1 lethal mutations, including severe hypotonia and polymicrogyria. In COS-1 cells, these mutations led to reduced expression in the plasma membrane by interference with the $\alpha-\beta$ interaction (Supplementary Fig. 8) leading to retention of immature protein in the endoplasmic reticulum and/or decreased stability. The ATP1A3 residue homologous to A2-R908 is targeted by an AHC mutation (A3R901T) (Holm et al., 2016) and the ATP1A2 residue homologous to A3-G893 is targeted by a hemiplegic migraine mutation (A2-G900R) (Deprez et al., 2008). The remaining three patients had less severe phenotypes consisting of mild-moderate intellectual disability with mild focal epilepsy (Pt1: A2-I293M; Pt5: A2-R593Q) or unilateral polymicrogyria and migraine (Pt15: A3-D887Y). The less severe phenotypes associated with A2-I293M and A2-R593Q may be related to their near normal expression level, which suggests a relatively stable protein, combined with retention of NKA pump activity, albeit with reduced maximal turnover rate.

Patient 21 carried the A3-F857del in 26.2\% of WES reads. Mosaicism for ATP1A3 mutations has been reported in a single AHC patient with VAF 44\% and a mild phenotype and in six asymptomatic parents of AHC probands (VAF <18.82\%) (Yang et al., 2019). The phenotype of Patient 21 was severe in spite of the mosaic state of the mutation which is likely to affect the interaction between $\mathrm{M} 7$ and the transmembrane helix of the $\beta$ subunit.

\section{Conclusion}

This series links profound early lethal phenotypes with COS-1 lethal mutations, but also reveals a continuum of phenotypic severity, in which both severe and milder phenotypes can be associated with mutations partially sparing NKA pump function, while polymicrogyria is distributed across mutations of variable effect. The molecular diagnostic yield of polymicrogyria is low, with multiple genes accounting for a small number of diagnoses each, and a relevant number of patients resulting from nongenetic, mainly vascular or infective, prenatal causes (Guerrini and Dobyns, 2014; Mirzaa et al., 2015; Park et al., 2020). The proportion of patients with polymicrogyria related to $A T P 1 A 2 / A 3$ remains unknown since 
available NGS studies on MCDs have been either performed with gene panels which included neither of the two genes (Oegema et al., 2020) or carrying out WES in small series in which no pathogenic variants in either gene emerged (Mirzaa et al., 2015; Wiszniewski et al., 2018). This study widens the clinical spectrum of ATP1A2/A3-opathies to include profound epilepsy phenotypes, with and without associated polymicrogyria. Inclusion of $A T P 1 A 2 / A 3$, in virtual panels used to interrogate WES/WGS data and in targeted-gene diagnostic panels for polymicrogyria and DEE will clarify the magnitude of their role in large series with these disorders.

\section{Contributors}

$\mathrm{RG}, \mathrm{BV}$ and WBD conceived the study and coordinated the experimental design of the research. AV, SB, EP, ZP, RSM, CB, GL, KLH, EEP, DM, EB, AVH, SS, VL, GiCi, CJC, GrCo, MS, $\mathrm{KC}, \mathrm{GM}, \mathrm{JCS}$, IES, WBD, and RG participated in genetic or clinical data acquisition and in the interpretation of results relevant to their domain of interest. HNN, RH and BV performed and interpreted functional experiments and structural analyses. RFH and AS acquired and interpreted neuropathological data. $\mathrm{AV}, \mathrm{RFH}, \mathrm{BV}, \mathrm{WBD}$, and $\mathrm{RG}$ drafted the manuscript and coordinated its finalisation. All authors critically reviewed the manuscript and approved the final draft.

\section{Acknowledgements}

We thank Nina Juste and Randi Scheel, Aarhus University, for expert technical assistance. We also thank Mrs Diana Bazan for her skilful technical assistance (Neuropathology). This work was generated within the European Reference Networks EpiCARE and ITHACA.

\section{Funding}

This work was supported by grants to B.V. from the Lundbeck Foundation (grant R223-2016595) and the Danish Medical Research Council (grant 7016-00193B), grants to R.G. from the European Union Seventh Framework Programme FP7/2013 under the project DESIRE (grant 602531) and the Italian Ministry of Health and Tuscany Region (grant RF-2013-02355240) and grants to I.E.S. from the National Health and Medical Research Council of Australia (grants APP1091593, APP1104831).

Competing interests: The authors declare no competing interests. 


\section{Appendix 1}

ATP1A2/A3-collaborators. Full details are provided in the Supplementary material.

Damien Sanlaville, Rani Sachdev, Ian Andrews, Francesco Mari, Anna Cavalli, Carmen Barba, Beatrice De Maria, Giampaolo Garani, Johannes R. Lemke, Mario Mastrangelo, Emily Tam, Elizabeth Donner, Helen Branson, Fabiola P. Monteiro, Fernando Kok, Katherine B Howell, Stephanie Leech, Heather Mefford, Alison Muir. 


\section{References:}

Arystarkhova E, Haq IU, Luebbert T, Mochel F, Saunders-Pullman R, Bressman SB, et al. Factors in the disease severity of ATP1A3 mutations: Impairment, misfolding, and allele competition. Neurobiol Dis 2019; 132: 104577.

Balestrini S, Mikati MA, Garcia-Roves RA, Carboni M, Hunanyan AS, Kherallah B, et al. Cardiac phenotype in ATP1A3 -related syndromes: A multicentre cohort study . Neurology 2020

Bassi MT, Bresolin N, Tonelli A, Nazos K, Crippa F, Baschirotto C, et al. A novel mutation in the ATP1A2 genes causes alternating hemiplegia of childhood. J Med Genet 2004

Blanco G. Na,K-ATPase subunit heterogeneity as a mechanism for tissue-specific ion regulation. Semin Nephrol 2005; 25: 292-303.

Blicher JU, Tietze A, Donahue MJ, Smith SA, Østergaard L. Perfusion and pH MRI in familial hemiplegic migraine with prolonged aura. Cephalalgia 2016; 36: 279-83.

Burgess R, Wang S, McTague A, Boysen KE, Yang X, Zeng Q, et al. The Genetic Landscape of Epilepsy of Infancy with Migrating Focal Seizures. Ann Neurol 2019; 86: 821-31.

De Carvalho Aguiar P, Sweadner KJ, Penniston JT, Zaremba J, Liu L, Caton M, et al. Mutations in the $\mathrm{Na}+\mathrm{K}+$-ATPase $\alpha 3$ gene ATP1A3 are associated with rapid-onset dystonia parkinsonism. Neuron 2004; 43: 169-75.

Chatron N, Cabet S, Alix E, Buenerd A, Cox P, Guibaud L, et al. A novel lethal recognizable polymicrogyric syndrome caused by ATP1A2 homozygous truncating variants. Brain 2019; 142: $3367-74$.

Dard R, Mignot C, Durr A, Lesca G, Sanlaville D, Roze E, et al. Relapsing encephalopathy with cerebellar ataxia related to an ATP1A3 mutation. Dev Med Child Neurol 2015; 57: 11836.

Deprez L, Weckhuysen S, Peeters K, Deconinck T, Claeys KG, Claes LRF, et al. Epilepsy as part of the phenotype associated with ATP1A2 mutations. Epilepsia 2008; 49: 500-8.

Friedrich T, Tavraz NN, Junghans C. ATP1A2 mutations in migraine: Seeing through the facets 
of an ion pump onto the neurobiology of disease. Front Physiol 2016; 7: 1-21.

Fry AE, Fawcett KA, Zelnik N, Yuan H, Thompson BAN, Shemer-Meiri L, et al. De novo mutations in GRIN1 cause extensive bilateral polymicrogyria. Brain 2018; 141: 698-712.

De Fusco M, Marconi R, Silvestri L, Atorino L, Rampoldi L, Morgante L, et al. Haploinsufficiency of ATP1A2 encoding the $\mathrm{Na}+/ \mathrm{K}+$ pump $\alpha 2$ subunit associated with familial hemiplegic migraine type 2. Nat Genet 2003; 33: 192-6.

Ghusayni R, Richardson JP, Uchitel J, Abdelnour E, McLean M, Prange L, et al. Magnetic resonance imaging volumetric analysis in patients with Alternating hemiplegia of childhood: A pilot study. Eur J Paediatr Neurol 2020; 26

Gressens P, Baes M, Leroux P, Lombet A, Van Veldhoven P, Janssen A, et al. Neuronal migration disorder in Zellweger mice is secondary to glutamate receptor dysfunction. Ann Neurol 2000; 48: 336-43.

Guerrini R, Dobyns WB. Malformations of cortical development: Clinical features and genetic causes. Lancet Neurol 2014; 13: 710-26.

Heinzen EL, Swoboda KJ, Hitomi Y, Gurrieri F, De Vries B, Tiziano FD, et al. De novo mutations in ATP1A3 cause alternating hemiplegia of childhood. Nat Genet 2012; 44: 1030-4.

Herrera VLM, Cova T, Sassoon D, Ruiz-Opazo N. Developmental cell-specific regulation of $\mathrm{Na}+-\mathrm{K}+-\mathrm{ATPase} \alpha 1-, \alpha 2-$, and $\alpha 3$-isoform gene expression. Am J Physiol - Cell Physiol 1994; 266: $35-5$.

Holm R, Toustrup-Jensen MS, Einholm AP, Schack VR, Andersen JP, Vilsen B. Neurological disease mutations of $\alpha 3 \mathrm{Na}+, \mathrm{K}+$-ATPase: Structural and functional perspectives and rescue of compromised function. Biochim Biophys Acta - Bioenerg 2016; 1857: 1807-28.

Ikeda K, Onaka T, Yamakado M, Nakai J, Ishikawa TO, Taketo MM, et al. Degeneration of the amygdala/piriform cortex and enhanced fear/anxiety behaviors in sodium pump $\alpha 2$ subunit (Atpla2)-deficient mice. J Neurosci 2003; 23: 4667-76.

Ikeda K, Onimaru H, Kawakami K. Knockout of sodium pump $\alpha 3$ subunit gene (Atp1a3-/-) results in perinatal seizure and defective respiratory rhythm generation. Brain Res 2017; 1666: 27-37. 
Kaplan JH. Biochemistry of Na,K-ATPase. Annu Rev Biochem 2002; 71: 511-35.

Li M, Jazayeri D, Corry B, McSweeney KM, Heinzen EL, Goldstein DB, et al. A functional correlate of severity in alternating hemiplegia of childhood. Neurobiol Dis 2015; 77: 88-93.

Liu J, Tong L, Song S, Niu Y, Li J, Wu X, et al. Novel and de novo mutations in pediatric refractory epilepsy. Mol Brain 2018; 11: 1-18.

Marzin P, Mignot C, Dorison N, Dufour L, Ville D, Kaminska A, et al. Early-onset encephalopathy with paroxysmal movement disorders and epileptic seizures without hemiplegic attacks: About three children with novel ATP1A3 mutations. Brain Dev 2018; 40: $768-74$.

McGrail KM, Phillips JM, Sweadner KJ. Immunofluorescent localization of three Na,KATPase isozymes in the rat central nervous system: Both neurons and glia can express more than one Na,K-ATPase. J Neurosci 1991; 11: 381-91.

McRae JF, Clayton S, Fitzgerald TW, Kaplanis J, Prigmore E, Rajan D, et al. Prevalence and architecture of de novo mutations in developmental disorders. Nature 2017; 542: 433-8.

Mirzaa GM, Conti V, Timms AE, Smyser CD, Ahmed S, Carter M, et al. Characterisation of mutations of the phosphoinositide-3-kinase regulatory subunit, PIK3R2, in perisylvian polymicrogyria: A next-generation sequencing study. Lancet Neurol 2015; 14: 1182-95.

Monteiro FP, Curry CJ, Hevner R, Elliott S, Fisher JH, Turocy J, et al. Biallelic loss of function variants in ATP1A2 cause hydrops fetalis, microcephaly, arthrogryposis and extensive cortical malformations. Eur J Med Genet 2020; 63: 103624.

Moseley AE, Lieske SP, Wetzel RK, James PF, He S, Shelly DA, et al. The Na,K-ATPase $\alpha 2$ isoform is expressed in neurons, and its absence disrupts neuronal activity in newborn mice. $\mathrm{J}$ Biol Chem 2003; 278: 5317-24.

Nielsen HN, Spontarelli K, Holm R, Andersen JP, Einholm AP, Artigas P, et al. Distinct effects of Q925 mutation on intracellular and extracellular $\mathrm{Na}+$ and $\mathrm{K}+$ binding to the $\mathrm{Na}+, \mathrm{K}+-$ ATPase. Sci Rep 2019; 9

Oegema R, Barakat TS, Wilke M, Stouffs K, Amrom D, Aronica E, et al. International consensus recommendations on the diagnostic work-up for malformations of cortical 
development. Nat Rev Neurol 2020

Paciorkowski AR, McDaniel SS, Jansen LA, Tully H, Tuttle E, Ghoneim DH, et al. Novel mutations in ATP1A3 associated with catastrophic early life epilepsy, episodic prolonged apnea, and postnatal microcephaly. Epilepsia 2015; 56: 422-30.

Panagiotakaki E, de Grandis E, Stagnaro M, Heinzen EL, Fons C, Sisodiya S, et al. Clinical profile of patients with ATP1A3 mutations in Alternating Hemiplegia of Childhood - A study of 155 patients. Orphanet J Rare Dis 2015; 10

Park K, Chapman T, Aldinger K, Mirzaa G, Zeiger J, Beck A, et al. The spectrum of brain malformations and disruptions in twins. Am J Med Genet Part A 2020; In press, Article ID: AJMGA61972; Article DOI: 10.1002/ajmg.a.61972

Parrini E, Marini C, Mei D, Galuppi A, Cellini E, Pucatti D, et al. Diagnostic Targeted Resequencing in 349 Patients with Drug-Resistant Pediatric Epilepsies Identifies Causative Mutations in 30 Different Genes. Hum Mutat 2017; 38: 216-25.

Pelzer N, Blom DE, Stam AH, Vijfhuizen LS, Hageman ATM, Van Vliet JA, et al. Recurrent coma and fever in familial hemiplegic migraine type 2. A prospective 15-year follow-up of a large family with a novel ATP1A2 mutation. Cephalalgia 2017; 37: 737-55.

Platzer K, Yuan H, Schütz H, Winschel A, Chen W, Hu C, et al. GRIN2B encephalopathy: Novel findings on phenotype, variant clustering, functional consequences and treatment aspects. J Med Genet 2017; 54: 460-70.

Prontera P, Sarchielli P, Caproni S, Bedetti C, Cupini LM, Calabresi P, et al. Epilepsy in hemiplegic migraine: Genetic mutations and clinical implications. Cephalalgia 2018; 38: 36173.

Reinhard L, Tidow H, Clausen MJ, Nissen P. Na+,K+-ATPase as a docking station: Proteinprotein complexes of the $\mathrm{Na}+, \mathrm{K}+-\mathrm{ATPase}$. Cell Mol Life Sci 2013; 70: 205-22.

Retterer K, Juusola J, Cho MT, Vitazka P, Millan F, Gibellini F, et al. Clinical application of whole-exome sequencing across clinical indications. Genet Med 2016; 18: 696-704.

Rosewich H, Ohlenbusch A, Huppke P, Schlotawa L, Baethmann M, Carrilho I, et al. The expanding clinical and genetic spectrum of ATP1A3-related disorders. Neurology 2014; 82: 
Rosewich H, Sweney MT, Debrosse S, Ess K, Ozelius L, Andermann E, et al. Research conference summary from the 2014 International Task Force on ATP1A3-related disorders. Neurol Genet 2017; 3

Rosewich H, Thiele H, Ohlenbusch A, Maschke U, Altmüller J, Frommolt P, et al. Heterozygous de-novo mutations in ATP1A3 in patients with alternating hemiplegia of childhood: A whole-exome sequencing gene-identification study. Lancet Neurol 2012; 11: 764-73.

Roth C, Freilinger T, Kirovski G, Dunkel J, Shah Y, Wilken B, et al. Clinical spectrum in three families with familial hemiplegic migraine type 2 including a novel mutation in the ATP1A2 gene. Cephalalgia 2014; 34: 183-90.

Roubergue A, Roze E, Vuillaumier-Barrot S, Fontenille MJ, Méneret A, Vidailhet M, et al. The multiple faces of the ATP1A3-related dystonic movement disorder. Mov Disord 2013; 28

Sasaki M, Ishii A, Saito Y, Hirose S. Progressive Brain Atrophy in Alternating Hemiplegia of Childhood. Mov Disord Clin Pract 2017; 4: 406-11.

Scheffer IE, Berkovic S, Capovilla G, Connolly MB, French J, Guilhoto L, et al. ILAE classification of the epilepsies: Position paper of the ILAE Commission for Classification and Terminology. Epilepsia 2017; 58: 512-21.

Severino M, Pisciotta L, Tortora D, Toselli B, Stagnaro M, Cordani R, et al. White matter and cerebellar involvement in alternating hemiplegia of childhood. J Neurol 2020; 267

Smith RS, Kenny CJ, Ganesh V, Jang A, Borges-Monroy R, Partlow JN, et al. Sodium Channel SCN3A (NaV1.3) Regulation of Human Cerebral Cortical Folding and Oral Motor Development. Neuron 2018; 99: 905-913.e7.

Smith RS, Walsh CA. Ion Channel Functions in Early Brain Development. Trends Neurosci 2020; 43: 103-14.

Sweadner KJ, Arystarkhova E, Penniston JT, Swoboda KJ, Brashear A, Ozelius LJ. Genotypestructure-phenotype relationships diverge in paralogs ATP1A1, ATP1A2, and ATP1A3. Neurol Genet 2019; 5 
Swoboda KJ, Kanavakis E, Xaidara A, Johnson JE, Leppert MF, Schlesinger-Massart MB, et al. Alternating hemiplegia of childhood or familial hemiplegic migraine?: A novel ATP1A2 mutation. Ann Neurol 2004; 55: 884-7.

Tavraz NN, Dürr KL, Koenderink JB, Freilinger T, Bamberg E, Dichgans M, et al. Impaired plasma membrane targeting or protein stability by certain ATP1A2 mutations identified in sporadic or familial hemiplegic migraine. Channels 2009; 3: 82-7.

Ueda K, Serajee F, Huq AM. Clinical benefit of NMDA receptor antagonists in a patient with ATP1A2 gene mutation. Pediatrics 2018; 141: S390-4.

De Vries B, Freilinger T, Vanmolkot KRJ, Koenderink JB, Stam AH, Terwindt GM, et al. Systematic analysis of three FHM genes in 39 sporadic patients with hemiplegic migraine. Neurology 2007; 69: 2170-6.

Weigand KM, Messchaert M, Swarts HGP, Russel FGM, Koenderink JB. Alternating Hemiplegia of Childhood mutations have a differential effect on $\mathrm{Na}+, \mathrm{K}+$-ATPase activity and ouabain binding. Biochim Biophys Acta - Mol Basis Dis 2014; 1842: 1010-6.

Wiszniewski W, Gawlinski P, Gambin T, Bekiesinska-Figatowska M, Obersztyn E, AntczakMarach D, et al. Comprehensive genomic analysis of patients with disorders of cerebral cortical development. Eur J Hum Genet 2018; 26

Yang X, Yang X, Chen J, Li S, Zeng Q, Huang AY, et al. ATP1A3 mosaicism in families with alternating hemiplegia of childhood. Clin Genet 2019; 96: 43-52.

Zaman T, Helbig KL, Clatot J, Thompson CH, Kang SK, Stouffs K, et al. SCN3A-Related Neurodevelopmental Disorder: A Spectrum of Epilepsy and Brain Malformation. Ann Neurol 2020; 88: 348-62. 


\section{Legend to figures}

Figure 1 - Brain MRI in 9 patients with polymicrogyria. From top to bottom, representative brain MRI findings in Patients 6, 7, 11-17 and 20 with an indication of the mutations and age at which imaging was performed. For each patient four cuts are presented, which include axial (column A), coronal (column B), sagittal through the sylvian fissure (column C), and sagittal through the midline (column D) images. Sequences are at 1.5 to $3 \mathrm{~T}$ and include T1-weighted, T2 weighted and fluid-attenuated inversion recovery (FLAIR) images. All patients have polymicrogyria with abnormal cortical infoldings and packed microgyri, combined with abnormal sulcation. A recurrent feature, present in all patients, is an abnormal sylvian fissure with thickened cortex, pronounced infolding and vertically oriented axis. These abnormalities are better visible in the A column (enlarged sylvian space), B column (infolding) and C column (cortical thickening and verticalized sylvian fissure). There are some differences in individual patients. In Patient 6, polymicrogyria is diffuse and more prominent posteriorly. In Patient 7, the cortical abnormality is asymmetrical, due to a smaller right hemisphere. In Patient 11 too, there is asymmetry with the right hemisphere being more severely affected and smaller. In Patients 13,14, 16 and 17, polymicrogyria is almost exclusively bilateral perisylvian, sparing the remaining cortex. In Patient 15 too, who is the mother of Patient 14, the cortical abnormality is perisylvian but unilateral only involving the right hemisphere. In Patient 17, there is diffuse polymicrogyria with more severe perisylvian involvement. In line D, midline cuts show thickening of the corpus callosum in Patients 6,14 and 15. There is some degree of vermian hypoplasia in Patients 7 and 17.

Figure 2 - Neuropathology of patient 11, age 4 months (A3-K764del). (A-C) Macroscopic views of the brain from posterior (A), right (B), and left (C) sides demonstrated overall small cerebral hemispheres, with externally apparent pachygyria (asterisks) involving pericentral cortex bilaterally. (D, E) Coronal slices through anterior (D) and middle (E) regions of the hemispheres showed thick cortex corresponding to externally apparent pachygyria (asterisks), and thin but intact genu of the corpus callosum (white arrow, D). (F, G) Histology of the brain slices demonstrated leptomeningeal glioneuronal heterotopia (black arrowheads) covering cortex that appeared externally pachygyric, but histologically showed features of 4-layered polymicrogyria, also seen in other cortical areas (black arrows). Callosal fibers did not cross the midline posteriorly, but instead formed Probst bundles (PB). 


\section{Figure 3 - Neuropathology of patient 23, age 1 day (A2-R279Gfs4*, homozygous).} Macroscopic brain abnormalities (A-F). (A) Left brain, lateral view. Blood vessels were congested; the leptomeninges contained subarachnoid haemorrhage (agonal) and were adherent to the brain surface. (B) Right hemisphere, medial view. The corpus callosum was thin (white arrow). (C) Brainstem and right cerebellum, medial view. The cerebellum was moderately hypoplastic. (D, E) Coronal slices of brain, level of amygdala (D) and anterior hippocampus (E). The basal ganglia and thalamus were moderately hypoplastic; the cortex was diffusely polymicrogyric, except for the hippocampi (black arrows), which appeared relatively wellformed. (F) The occipital lobes (coronal slices) exhibited polymicrogyria and moderate colpocephaly. Histopathology (H\&E; G-N) (G) Frontal cortex, coronal section (midline right). The cortex was diffusely polymicrogyric, had an attached external rind of leptomeningeal glioneuronal heterotopia, and contained focal calcifications (arrows). The corpus callosum (CC) was small, but crossed the midline. (H) Medial temporal lobe, coronal section. The hippocampus was well-formed, including the dentate gyrus (DG), while neocortex was polymicrogyric. Focal calcifications (arrows) were noted in deep white matter. (I) Leptomeningeal glioneuronal heterotopia (Het) covered much of the cortical surface. Note focal parenchymal calcifications (arrows). (J) Leptomeninges with focal calcifications (arrows). (K) Cortical blood vessel with mineralized walls (arrows). (L) Meningeal blood vessel with medial calcifications (arrows), and intimal hyperplasia possibly due to thrombosis and recanalization. Scale bars: (A) $1 \mathrm{~cm}$ for (A-F); (G), $1 \mathrm{~mm}$ for (G,H); (I), $100 \mu \mathrm{m}$ for (I,J); $50 \mu \mathrm{m}$ for (K,L).

\section{Figure 4: Overview of the structural location of ATP1A2 and ATP1A3 disease mutations}

\section{in the cohort}

The NKA $\alpha$-subunit consists of three major cytoplasmic domains A ("actuator"), N ("nucleotide-binding"), and P ("phosphorylation") linked to a membrane domain composed of ten transmembrane helices (M1-M10) with their intervening loops on both membrane sides. The $\beta$-subunit (PDB ID 3WGV, chain B, teal color) consists of a transmembrane helix $\beta \mathrm{m}$ and an extracellular domain that interacts with the $\alpha$-subunit loop connecting M7 and M8 (L7-8 loop). Amino acid residues targeted by the mutations in the cohort are shown in blue (ATP1A2 mutations) and red (ATP1A3 mutations) in the structure of the ATP1A1 $\alpha$-subunit in E1 form (PDB ID 3WGV, chain A, grey color) with $3 \mathrm{Na}^{+}$(golden spheres labelled I, II, and III according to common nomenclature) and $\mathrm{ADP}$ with phosphate analog $\mathrm{AlF}_{4}$ and the phosphorylated aspartate in green. 


\section{Figure 5: Relative phosphorylation/expression and turnover rate for ATPase activity with} NKA reaction cycle

(A) Phosphorylation level of stably expressed ATP1A2 and ATP1A3 wild type and mutants. The phosphorylation level ( $\mathrm{pmol} / \mathrm{mg}$ total plasma membrane protein) is shown in \% of wild type. Phosphorylation was determined under stoichiometric conditions, thus reflecting the expression level (“active site concentration"). (B) Turnover rate $\left(\mathrm{min}^{-1}\right)$ determined by relating the maximum NKA activity to the phosphorylation level. (C) Phosphorylation level of transiently expressed ATP1A2 and ATP1A3 wild type and mutants determined as described for (A). The NKA activity per mg total plasma membrane protein, calculated by multiplying the turnover rate by the phosphorylation level, was 17\% (A2-I293M), 36\% (A2-R593Q), 22\% (A2R908Q), 30\% (A3-P972del), and 33\% (A3-D887Y), relative to wild-type, indicating that the function of these mutants was substantially impaired despite their ability to support cell growth. (D) Reaction cycle of NKA. E1 and E1P are $\mathrm{Na}^{+}$selective conformations, whereas E2 and E2P are $\mathrm{K}^{+}$selective conformations. Phosphorylation by ATP (indicated by "P”). The cytoplasmic and extracellular sides are indicated by c and e, respectively, see detailed description in supplementary legend to figure 5 (Supplementary Materials) (Holm et al., 2016).

\section{Figure 6: $\mathrm{Na}^{+}$and $\mathrm{K}^{+}$affinity of ATP1A2/A3 mutants}

(A) $\mathrm{Na}^{+}$affinity determined from $\mathrm{Na}^{+}$activation of phosphorylation. The binding of three $\mathrm{Na}^{+}$ to the E1 form activates phosphorylation from ATP. Phosphorylation was measured at the indicated concentrations of $\mathrm{Na}^{+}$. Symbols with error bars represent mean $\pm \mathrm{SD}$. Each line represents the best fit of a Hill equation. The apparent $\mathrm{Na}^{+}$affinities $\left(\mathrm{K}_{0.5}\right.$ values $)$ and Hill coefficients $\left(\mathrm{n}_{\mathrm{H}}\right)$ were as follows. Stable expression (upper panels): A2-wt (wild-type), $534 \pm$ $52 \mu \mathrm{M}\left(\mathrm{n}_{\mathrm{H}}=2.0\right) ;$ A2-I293M, $2790 \pm 592 \mu \mathrm{M}\left(\mathrm{n}_{\mathrm{H}}=1.7\right)$; A2-R593Q, $384 \pm 24 \mu \mathrm{M}\left(\mathrm{n}_{\mathrm{H}}=2.6\right)$; A2-R908Q, $601 \pm 81 \mu \mathrm{M}\left(\mathrm{n}_{\mathrm{H}}=1.7\right)$; A3-wt, $916 \pm 105 \mu \mathrm{M}\left(\mathrm{n}_{\mathrm{H}}=2.4\right)$; A3-D887Y, $1260 \pm 93$ $\mu \mathrm{M}\left(\mathrm{n}_{\mathrm{H}}=2.1\right)$; A3-P972del, $1331 \pm 303 \mu \mathrm{M}\left(\mathrm{n}_{\mathrm{H}}=1.2\right)$. Transient expression (lower panels): A2-wt, $595 \pm 80 \mu \mathrm{M}\left(\mathrm{n}_{\mathrm{H}}=2.5\right)$; A2-G366A, 3079 $\pm 192 \mu \mathrm{M}\left(\mathrm{n}_{\mathrm{H}}=1.3\right)$; A3-wt, $1120 \pm 127 \mu \mathrm{M}$ $\left(\mathrm{n}_{\mathrm{H}}=2.1\right)$; A3-L292R, $3013 \pm 254 \mu \mathrm{M}\left(\mathrm{n}_{\mathrm{H}}=1.9\right)$; A3-G316V, $272 \pm 47 \mu \mathrm{M}\left(\mathrm{n}_{\mathrm{H}}=1.3\right)$. The apparent $\mathrm{Na}^{+}$affinity was reduced 3- to 5-fold ( $\mathrm{K}_{0.5}$ increased) for A2-I293M, A2-G366A, and A3-L292R, and increased 4-fold for A3-G316V ( $\mathrm{K}_{0.5}$ decreased). For A3-P972del, the cooperativity of $\mathrm{Na}^{+}$binding at the three sites was severely reduced (Hill coefficient 1.2 vs. 2.4 for the wild-type). (B) $\mathrm{K}^{+}$affinity determined from $\mathrm{K}^{+}$inhibition of phosphorylation. Accumulation of phosphoenzyme is inhibited by $\mathrm{K}^{+}$, because $\mathrm{K}^{+}$activates dephosphorylation of E2P and competes with $\mathrm{Na}^{+}$for binding to E1 (cf. reaction cycle in Fig. 5). Phosphorylation 
was measured at the indicated concentrations of $\mathrm{K}^{+}$on transiently expressed NKA enzyme. Symbols with error bars represent mean \pm SD. Each line represents the best fit of the Hill equation for inhibitory ligand binding (Nielsen et al., 2019). The apparent $\mathrm{K}^{+}$affinities $\left(\mathrm{K}_{0.5}\right.$ values) were as follows: A2-wt, $188 \pm 43 \mu \mathrm{M}$; A2-G366A, >100 mM; A3-wt, $281 \pm 63 \mu \mathrm{M}$; A3-L292R, $4570 \pm 700 \mu \mathrm{M}$; A3-G316V, >10 mM. Hence, A3-L292R and A3-G316V showed 16- and $>35$-fold reduction of apparent $\mathrm{K}^{+}$affinity, and A2-G366A was totally insensitive to $\mathrm{K}^{+}$.

\section{Figure 7 - Distribution of ATP1A2/A3 mutations across their associated phenotypes.}

The pie charts show the distribution of heterozygous mutations of ATP1A2 (left) and ATP1A3 (right) across their associated phenotypes. We identified 92 ATP1A2 and 136 ATP1A3 heterozygous mutations in the Human Gene Mutation Database (https://portal.biobaseinternational.com), and stratified them based on the reported phenotypes, adding to previously published data the 11 novel mutations ( 2 in ATP1A2 and 9 in ATP1A3) we reported in this series. Collectively, up to $5 \%$ of ATP1A2 (6/94) and 12\% of ATP1A3 (18/145) mutations can be associated with developmental and epileptic encephalopathy. Polymicrogyria can be associated with $1 \%$ of $A T P 1 A 2$ and $5.5 \%$ of $A T P 1 A 3$ mutations (yellow-dashed area). Abbreviations: AHC, alternating hemiplegia of childhood; DYT, dystonia; DEE, developmental and epileptic encephalopathy (this term includes epileptic encephalopathies and developmental encephalopathies with epilepsy); FHM, familial hemiplegic migraine; PMG, polymicrogyria; RDP, rapid-onset dystonia-parkinsonism; RP, rare phenotypes (this sub-group includes CAPOS, RECA, ataxia and non-specified nervous system abnormalities). 
Table 1

\begin{tabular}{|c|c|c|c|c|c|c|c|c|c|}
\hline & \multicolumn{6}{|c|}{$A T P 1 A 2$, heterozygous } & \multicolumn{3}{|c|}{$A T P 1 A 3$, heterozygous } \\
\hline $\begin{array}{l}\text { Patient } \\
\text { Nr/sex }\end{array}$ & $1 / \mathbf{M}$ & $2 / \mathbf{M}$ & $3 / \mathbf{M}$ & $4 / F$ & 5/M & 6/M & $7 / \mathrm{F}$ & $8 / F$ & $9 / F$ \\
\hline $\begin{array}{c}\text { Mutation } \\
\text { (protein)/ } \\
\text { abbreviati } \\
\text { on }\end{array}$ & $\begin{array}{c}\mathrm{p} .(\text { Ile29 } \\
\text { 3Met)/A } \\
2- \\
\text { I293M }\end{array}$ & $\begin{array}{c}\text { p.(Cys34 } \\
1 \mathrm{Phe}) / \mathrm{A} \\
2-\mathrm{C} 341 \mathrm{~F}\end{array}$ & $\begin{array}{c}\text { p.(Gly36 } \\
\text { 6Ala)**/ } \\
\text { A2- } \\
\text { G366A }\end{array}$ & $\begin{array}{c}\text { p.(Gly36 } \\
\text { 6Ala)**/ } \\
\text { A2- } \\
\text { G366A }\end{array}$ & $\begin{array}{c}\text { p. }(\text { Arg59 } \\
\text { 3Gln)/A } \\
2- \\
\text { R593Q }\end{array}$ & $\begin{array}{c}\text { p.(Arg90 } \\
8 \mathrm{Gln}) / \mathrm{A} \\
2- \\
\text { R908Q }\end{array}$ & $\begin{array}{l}\text { p.(Leu29 } \\
\text { 2Arg)/A } \\
\text { 3-L292R }\end{array}$ & $\begin{array}{c}\text { p.(Gly31 } \\
\text { 6Val)/A } \\
\text { 3- } \\
\text { G316V }\end{array}$ & $\begin{array}{c}\text { p.(Ser361 } \\
\text { Pro)**/A } \\
3-S 361 P\end{array}$ \\
\hline $\begin{array}{c}\text { Inherited/ } \\
\text { De } \\
\text { novo/type } \\
\text { of } \\
\text { analysis } \\
\end{array}$ & $\begin{array}{c}\text { De } \\
\text { novo/W } \\
\text { ES }\end{array}$ & $\begin{array}{c}\text { De } \\
\text { novo/TG } \\
\text { P }\end{array}$ & $\begin{array}{c}\text { De } \\
\text { novo/WE } \\
\mathrm{S}\end{array}$ & $\begin{array}{c}\text { De } \\
\text { novo/WE } \\
\text { S }\end{array}$ & $\begin{array}{c}\text { De } \\
\text { novo/TG } \\
\text { P }\end{array}$ & $\begin{array}{c}\text { De } \\
\text { novo/W } \\
\text { ES }\end{array}$ & $\begin{array}{c}\text { De } \\
\text { novo/W } \\
\text { GS }\end{array}$ & $\begin{array}{c}\text { De } \\
\text { novo/W } \\
\text { ES }\end{array}$ & $\begin{array}{c}\text { De } \\
\text { novo/WE } \\
\mathrm{S}\end{array}$ \\
\hline 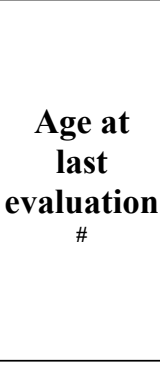 & $12 y$ & $5 y$ & $\begin{array}{c}1 \mathrm{y}, \\
\text { deceased } \\
\text { (respirato } \\
\text { ry } \\
\text { complicat } \\
\text { ions of } \\
\text { status } \\
\text { epilepticu } \\
\text { s) }\end{array}$ & $\begin{array}{l}1 \mathrm{~m} 3 \mathrm{w}, \\
\text { deceased } \\
\text { (respirato } \\
\text { ry } \\
\text { complicat } \\
\text { ions of } \\
\text { status } \\
\text { epilepticu } \\
\text { s) }\end{array}$ & $8 y$ & $11 y$ & $\begin{array}{c}2 \mathrm{~m}, \\
\text { deceased } \\
\text { (respirat } \\
\text { ory } \\
\text { complica } \\
\text { tions of } \\
\text { status } \\
\text { epileptic } \\
\text { us) }\end{array}$ & $6 y$ & $7 y$ \\
\hline $\begin{array}{c}\text { Prenatal/ } \\
\text { perinatal } \\
\text { findings }\end{array}$ & $\begin{array}{c}\text { Unrema } \\
\text { rkable }\end{array}$ & $\begin{array}{c}\text { Unremar } \\
\text { kable }\end{array}$ & $\begin{array}{c}\text { Dizygotic } \\
\text { twin } \\
\text { (twin } \\
\text { unaffecte } \\
\text { d) }\end{array}$ & $\begin{array}{l}\text { Caesarea } \\
\mathrm{n} \text { section } \\
\text { at } 38 \mathrm{w} \\
\text { because } \\
\text { of } \\
\text { oligohydr } \\
\text { amnios }\end{array}$ & $\begin{array}{c}\text { Unremar } \\
\text { kable }\end{array}$ & $\begin{array}{c}\text { Unremar } \\
\text { kable }\end{array}$ & $\begin{array}{c}\text { Antenata } \\
1 \\
\text { 'hiccoug } \\
\text { h'. Apgar } \\
\text { 3/6/6 }\end{array}$ & $\begin{array}{c}\text { Unremar } \\
\text { kable }\end{array}$ & $\begin{array}{c}\text { Unremar } \\
\text { kable }\end{array}$ \\
\hline $\begin{array}{c}\text { Age at } \\
\text { seizure } \\
\text { onset/ } \\
\text { type/sever } \\
\text { ity }\end{array}$ & $\begin{array}{c}18 \mathrm{~m} / \text { foc } \\
\text { al } \\
\text { dyscogn } \\
\text { itive, } \\
\text { left } \\
\text { unilater } \\
\text { al } \\
\text { clonic/i } \\
\text { nitially } \\
\text { frequent } \\
\text {; seizure } \\
\text { free } \\
\text { since } \\
\text { age } 5 y\end{array}$ & $\begin{array}{l}\text { 3w/tonic } \\
\text { spasms, } \\
\text { migratin } \\
\text { g focal } \\
\text { seizures/ } \\
\text { severe }\end{array}$ & $\begin{array}{l}1 \mathrm{~m} / \text { prolo } \\
\text { nged } \\
\text { migrating } \\
\text { focal } \\
\text { seizures } \\
\text { with } \\
\text { apnea, } \\
\text { cyanosis } \\
\text { and } \\
\text { hypertonu } \\
\text { s } \\
\text { evolving } \\
\text { to super- } \\
\text { refractory } \\
\text { status } \\
\text { epilepticu } \\
\text { s }\end{array}$ & $\begin{array}{l}\text { 10d/prolo } \\
\text { nged } \\
\text { migrating } \\
\text { focal } \\
\text { seizures } \\
\text { evolving } \\
\text { to super- } \\
\text { refractory } \\
\text { status } \\
\text { epilepticu } \\
\text { s }\end{array}$ & $\begin{array}{l}\text { 8y/gener } \\
\text { alised } \\
\text { TC with } \\
\text { prolonge } \\
\text { d post- } \\
\text { ictal } \\
\text { confusio } \\
\text { n/recent } \\
\text { onset }\end{array}$ & $\begin{array}{c}8 \mathrm{y} / \text { focal } \\
\text { onset } \\
\text { with } \\
\text { bilateral } \\
\text { posturin } \\
\text { g/rare }\end{array}$ & \begin{tabular}{|} 
At \\
birth/mi \\
grating \\
focal \\
seizures \\
with \\
apnea/ \\
almost \\
continuo \\
us \\
seizures, \\
status \\
epileptic \\
us/sever \\
e
\end{tabular} & $\begin{array}{c}4 \mathrm{y} / \\
\text { migratin } \\
\mathrm{g} \text { focal } \\
\text { and } \\
\text { generalis } \\
\text { ed } \\
\text { seizures/ } \\
\text { severe }\end{array}$ & $\begin{array}{l}5 \mathrm{~m} / \text { focal } \\
\text { temporal } \\
\text { status } \\
\text { epilepticu } \\
\text { s with } \\
\text { ictal } \\
\text { vomiting } \\
\text { at onset, } \\
\text { hemiclon } \\
\text { ic/severe }\end{array}$ \\
\hline $\begin{array}{c}\text { Interictal } \\
\text { EEG }\end{array}$ & $\begin{array}{c}\text { Bilateral } \\
\text { occipital } \\
\text { discharg } \\
\text { es }\end{array}$ & $\begin{array}{c}\text { Slow } \\
\text { backgro } \\
\text { und, } \\
\text { multifoc } \\
\text { al sharp } \\
\text { waves }\end{array}$ & $\begin{array}{c}\text { Burst } \\
\text { suppressi } \\
\text { on, } \\
\text { multifoca } \\
1 \\
\text { discharge } \\
\text { s }\end{array}$ & $\begin{array}{c}\text { Slow } \\
\text { backgrou } \\
\text { nd } \\
\text { activity, } \\
\text { multifoca } \\
1 \\
\text { discharge } \\
\text { s }\end{array}$ & NA & $\begin{array}{c}\text { Continu } \\
\text { ous } \\
\text { bilateral } \\
\text { fronto- } \\
\text { temporal } \\
\text { and } \\
\text { multifoc } \\
\text { al } \\
\text { discharg } \\
\text { es } \\
\end{array}$ & $\begin{array}{c}\text { Multifoc } \\
\text { al } \\
\text { discharg } \\
\text { es }\end{array}$ & $\begin{array}{c}\text { Slow } \\
\text { backgro } \\
\text { und, } \\
\text { multifoc } \\
\text { al } \\
\text { discharg } \\
\text { es }\end{array}$ & $\begin{array}{c}\text { Slow } \\
\text { backgrou } \\
\text { nd, } \\
\text { multifoca } \\
1 \\
\text { discharge } \\
\text { s }\end{array}$ \\
\hline
\end{tabular}




\begin{tabular}{|c|c|c|c|c|c|c|c|c|c|}
\hline & \multicolumn{6}{|c|}{$A T P 1 A 2$, heterozygous } & \multicolumn{3}{|c|}{$A T P 1 A 3$, heterozygous } \\
\hline $\begin{array}{l}\text { Patient } \\
\text { Nr/sex }\end{array}$ & $1 / \mathbf{M}$ & $2 / \mathbf{M}$ & 3/M & $4 / F$ & $5 / \mathbf{M}$ & 6/M & $7 / F$ & $8 / \mathbf{F}$ & $9 / \mathbf{F}$ \\
\hline $\begin{array}{c}\text { Brain } \\
\text { MRI: } \\
\text { age/findin } \\
\text { gs }\end{array}$ & $\begin{array}{c}18 \\
\mathrm{~m} / \mathrm{bilate} \\
\text { ral } \\
\text { peritrigo } \\
\text { nal high } \\
\text { signal } \\
\text { intensity }\end{array}$ & $\begin{array}{c}1 \mathrm{~m} / \text { thin } \\
\text { corpus } \\
\text { callosum } \\
\text {, frontal } \\
\text { and } \\
\text { cerebella } \\
\mathrm{r} \\
\text { atrophy; } \\
13 \mathrm{~m} / \\
\text { worseni } \\
\text { ng of } \\
\text { atrophy, } \\
\text { dilated } \\
\text { ventricle } \\
\text { s }\end{array}$ & $\begin{array}{c}1 \mathrm{~m} / \text { norm } \\
\text { al }\end{array}$ & $\begin{array}{c}1 \mathrm{~m} / \text { norm } \\
\text { al }\end{array}$ & $\begin{array}{c}\text { 8y/norm } \\
\text { al }\end{array}$ & $\begin{array}{c}\text { 3y/bilate } \\
\text { ral } \\
\text { perisylvi } \\
\text { an } \\
\text { polymicr } \\
\text { ogyria, } \\
\text { thickene } \\
\text { d corpus } \\
\text { callosum }\end{array}$ & $\begin{array}{l}\text { 4d/exten } \\
\text { sive } \\
\text { bilateral, } \\
\text { perisylvi } \\
\text { an } \\
\text { predomi } \\
\text { nant, } \\
\text { polymicr } \\
\text { ogyria }\end{array}$ & $\begin{array}{c}7 \mathrm{~m} / \text { diffu } \\
\text { se brain, } \\
\text { brainste } \\
\mathrm{m} \text { and } \\
\text { cerebella } \\
\mathrm{r} \\
\text { atrophy, } \\
\text { thin } \\
\text { corpus } \\
\text { callosu } \\
\text { m; } 3 \\
\text { y/worse } \\
\text { ning of } \\
\text { atrophy }\end{array}$ & $\begin{array}{c}8 \mathrm{~m} \text { and } \\
30 \mathrm{~m} / \mathrm{nor} \\
\mathrm{mal}\end{array}$ \\
\hline $\begin{array}{c}\text { Microcep } \\
\text { haly } \\
\text { (OFC <- } \\
\text { 2SD) } \\
\end{array}$ & No & No & No & $\begin{array}{c}\text { Yes, } \\
\text { postnatal } \\
(-2 \mathrm{SD})\end{array}$ & No & $\begin{array}{c}\text { Yes, } \\
\text { postnatal } \\
(-2.5 \mathrm{SD})\end{array}$ & $\begin{array}{c}\text { Yes, } \\
\text { postnatal } \\
(-2.4 \mathrm{SD})\end{array}$ & No & $\begin{array}{c}\text { Yes, } \\
\text { postnatal } \\
(-2.8 \mathrm{SD})\end{array}$ \\
\hline $\begin{array}{c}\text { Other } \\
\text { Neurologi } \\
\text { cal } \\
\text { Features }\end{array}$ & \begin{tabular}{|c} 
Moderat \\
$\mathrm{e}$ \\
develop \\
mental \\
delay/I \\
$\mathrm{D}$, \\
ADHD, \\
dubious \\
episodes \\
of left \\
sided \\
hemipar \\
esis \\
likely \\
postictal
\end{tabular} & $\begin{array}{c}\text { Severe } \\
\text { develop } \\
\text { mental } \\
\text { delay, } \\
\text { nonverb } \\
\text { al, } \\
\text { severe } \\
\text { axial } \\
\text { hypotoni } \\
\text { a, } \\
\text { dyskinet } \\
\text { ic } \\
\text { quadripa } \\
\text { resis }\end{array}$ & $\begin{array}{l}\text { Profound } \\
\text { delay, } \\
\text { severe } \\
\text { hypotonia }\end{array}$ & $\begin{array}{l}\text { Profound } \\
\text { delay, } \\
\text { severe } \\
\text { hypotonia } \\
\text {, poor eye } \\
\text { contact }\end{array}$ & No & $\begin{array}{c}\text { Moderat } \\
\text { e } \\
\text { develop } \\
\text { mental } \\
\text { delay, } \\
\text { hypotoni } \\
\text { c } \\
\text { quadripa }\end{array}$ & $\begin{array}{l}\text { Profoun } \\
\text { d delay, } \\
\text { severe } \\
\text { hypotoni } \\
\text { a }\end{array}$ & $\begin{array}{l}\text { Profoun } \\
\text { d delay, } \\
\text { hypotoni } \\
\text { c } \\
\text { quadripa } \\
\text { resis }\end{array}$ & $\begin{array}{c}\text { Moderate } \\
\text { developm } \\
\text { ental } \\
\text { delay }\end{array}$ \\
\hline $\begin{array}{l}\text { Additiona } \\
\text { I Clinical } \\
\text { findings } \\
\text { and ECG }\end{array}$ & $\begin{array}{c}\text { Sleep } \\
\text { apnea } \\
\text { (obstruc } \\
\text { tive?/ } \\
\text { central? } \\
\text { ), cyclic } \\
\text { vomitin } \\
\text { g; ECG } \\
\text { unavaila } \\
\text { ble }\end{array}$ & $\begin{array}{c}\text { No; } \\
\text { ECG: } \\
\text { normal }\end{array}$ & $\begin{array}{c}\text { No; ECG: } \\
\text { normal }\end{array}$ & $\begin{array}{c}\text { Down } \\
\text { syndrome } \\
\text { with } \\
\text { complex } \\
\text { congenita } \\
1 \text { heart } \\
\text { disease; } \\
\text { ECG: } \\
\text { normal }\end{array}$ & $\begin{array}{c}\text { No; } \\
\text { ECG } \\
\text { unavaila } \\
\text { ble }\end{array}$ & $\begin{array}{c}\text { No; } \\
\text { ECG: } \\
\text { normal }\end{array}$ & $\begin{array}{c}\text { Right } \\
\text { ventricul } \\
\text { ar } \\
\text { hypertro } \\
\text { phy }\end{array}$ & $\begin{array}{c}\text { No; } \\
\text { ECG: } \\
\text { normal }\end{array}$ & $\begin{array}{c}\text { No; } \\
\text { ECG: } \\
\text { normal }\end{array}$ \\
\hline $\begin{array}{l}\text { Novel/Ref } \\
\text {. (for } \\
\text { previously } \\
\text { reported } \\
\text { mutations } \\
\text { ) }\end{array}$ & $\begin{array}{c}\text { (Ueda et } \\
\text { al., } \\
\text { 2018) }\end{array}$ & Novel & $\begin{array}{l}\text { (Parrini et } \\
\text { al., 2017) }\end{array}$ & $\begin{array}{l}\text { (Parrini et } \\
\text { al., 2017) }\end{array}$ & Novel & $\begin{array}{c}(\mathrm{De} \\
\text { Vries et } \\
\text { al., } \\
2007)\end{array}$ & Novel & Novel & Novel \\
\hline
\end{tabular}


Table 1 (continues)

\begin{tabular}{|c|c|c|c|c|c|c|c|c|c|}
\hline \multirow[b]{2}{*}{$\begin{array}{l}\text { Patient } \\
\text { Nr/sex }\end{array}$} & \multicolumn{9}{|c|}{$A T P 1 A 3$, heterozygous } \\
\hline & $10 / M$ & $11 / F$ & $12 / \mathrm{M}$ & $13 / \mathbf{M}$ & 14/M & $15 / F$ & $16 / M$ & $17 / F$ & $18 / F$ \\
\hline $\begin{array}{c}\text { Mutation } \\
\text { (protein)/a } \\
\text { bbreviatio } \\
\text { n }\end{array}$ & $\begin{array}{l}\text { p.(Asp60 } \\
\text { 9Tyr)/A3 } \\
\text {-D609Y }\end{array}$ & $\begin{array}{c}\text { p.(Lys76 } \\
\text { 4del)/A3 } \\
- \\
\text { K764del } \\
\end{array}$ & $\begin{array}{l}\text { p.(Pro77 } \\
\text { 5Arg)/A } \\
\text { 3-P775R }\end{array}$ & $\begin{array}{l}\text { p. }(\text { Asp80 } \\
1 \text { Asn }) / A \\
3-D 801 N\end{array}$ & $\begin{array}{l}\text { p.(Asp88 } \\
7 \mathrm{Tyr}) / \mathrm{A} 3 \\
-\mathrm{D} 887 \mathrm{Y}\end{array}$ & $\begin{array}{l}\text { p. }(\text { Asp88 } \\
7 \mathrm{Tyr}) / \mathrm{A} 3 \\
-\mathrm{D} 887 \mathrm{Y}\end{array}$ & $\begin{array}{l}\text { p.(Leu88 } \\
\text { 8Pro)/A3 } \\
\text {-L888P }\end{array}$ & $\begin{array}{c}\text { p.(Gly89 } \\
\text { 3Trp)/A } \\
\text { 3- } \\
\text { G893W }\end{array}$ & $\begin{array}{c}\text { p.(Pro97 } \\
\text { 2del)/A3 } \\
- \\
\text { P972del }\end{array}$ \\
\hline $\begin{array}{c}\text { Inherited/ } \\
\text { De } \\
\text { novo/type } \\
\text { of analysis }\end{array}$ & $\begin{array}{c}\text { De } \\
\text { novo/WE } \\
\mathrm{S}\end{array}$ & $\begin{array}{c}\text { De } \\
\text { novo/W } \\
\text { ES }\end{array}$ & $\begin{array}{c}\text { De } \\
\text { novo/W } \\
\text { ES }\end{array}$ & $\begin{array}{c}\text { De } \\
\text { novo/WE } \\
\text { S }\end{array}$ & $\begin{array}{c}\text { Inherited } \\
\text { (mat)/W } \\
\text { ES }\end{array}$ & $\begin{array}{c}\text { Inherited } \\
\text { (pat)/WE } \\
\text { S }\end{array}$ & $\begin{array}{c}\text { De } \\
\text { novo/W } \\
\text { ES }\end{array}$ & $\begin{array}{c}\text { De } \\
\text { novo/W } \\
\text { ES }\end{array}$ & $\begin{array}{c}\text { De } \\
\text { novo/W } \\
\text { ES }\end{array}$ \\
\hline $\begin{array}{c}\text { Age at last } \\
\text { evaluation } \\
\#\end{array}$ & $\begin{array}{c}14 y, \\
\text { deceased } \\
\text { (respirato } \\
\text { ry } \\
\text { complica } \\
\text { tions of } \\
\text { status } \\
\text { epileptic } \\
\text { us) } \\
\end{array}$ & $\begin{array}{c}4 \mathrm{~m}, \\
\text { deceased } \\
\text { (respirat } \\
\text { ory } \\
\text { complic } \\
\text { ations of } \\
\text { status } \\
\text { epileptic } \\
\text { us) }\end{array}$ & $12 y$ & $3 y$ & $7 y$ & $32 y$ & $28 y$ & $\begin{array}{c}10 \mathrm{~d}, \\
\text { deceased } \\
\text { (respirat } \\
\text { ory } \\
\text { complica } \\
\text { tions of } \\
\text { status } \\
\text { epileptic } \\
\text { us) }\end{array}$ & $\begin{array}{c}2 \mathrm{y} 6 \mathrm{~m}, \\
\text { decease } \\
\mathrm{d} \\
\text { (intercur } \\
\text { rent } \\
\text { respirato } \\
\text { ry } \\
\text { infection } \\
\text { ) }\end{array}$ \\
\hline $\begin{array}{c}\text { Prenatal/p } \\
\text { erinatal } \\
\text { findings }\end{array}$ & $\begin{array}{l}\text { Unremar } \\
\text { kable }\end{array}$ & $\begin{array}{l}\text { Prenatal } \\
\text { US at } \\
32 \mathrm{w}: \\
\text { microgn } \\
\text { athia, } \\
\text { prefront } \\
\text { al } \\
\text { edema, } \\
\text { reduced } \\
\text { fetal } \\
\text { moveme } \\
\text { nts. } \\
\text { Apgar } \\
2 / 5 / 7\end{array}$ & $\begin{array}{l}\text { Unremar } \\
\text { kable }\end{array}$ & $\begin{array}{l}\text { Unremar } \\
\text { kable }\end{array}$ & $\begin{array}{l}\text { Unremar } \\
\text { kable }\end{array}$ & $\begin{array}{c}\text { Unremar } \\
\text { kable }\end{array}$ & $\begin{array}{l}\text { Unremar } \\
\text { kable }\end{array}$ & $\begin{array}{l}\text { Unremar } \\
\text { kable }\end{array}$ & $\begin{array}{c}\text { Born at } \\
\text { term by } \\
\text { Caesare } \\
\text { an } \\
\text { section } \\
\text { because } \\
\text { of } \\
\text { maternal } \\
\text { bleeding }\end{array}$ \\
\hline $\begin{array}{c}\text { Age at } \\
\text { seizure } \\
\text { onset/ } \\
\text { type/severi } \\
\text { ty }\end{array}$ & $\begin{array}{l}2 \mathrm{~m} / \text { focal } \\
\text { clonic- } \\
\text { tonic, } \\
\text { tonic } \\
\text { posturing } \\
\text { and } \\
\text { sweating, } \\
\text { dyscogni } \\
\text { tive with } \\
\text { nystagm } \\
\text { us,episod } \\
\text { es of } \\
\text { status } \\
\text { epileptic } \\
\text { us/severe }\end{array}$ & $\begin{array}{c}9 \mathrm{~d} / \\
\text { migratin } \\
\mathrm{g} \text { focal } \\
\text { seizures } \\
\text { with } \\
\text { apnea, } \\
\text { hyperton } \\
\text { us, and } \\
\text { eye } \\
\text { deviatio } \\
\text { n,super- } \\
\text { refractor } \\
\text { y status } \\
\text { epileptic } \\
\text { us/sever } \\
\text { e }\end{array}$ & $\begin{array}{l}2 y / \text { tonic } \\
\text { seizures } \\
\text { with } \\
\text { cyanosis/ } \\
\text { severe }\end{array}$ & $\begin{array}{c}\text { At birth/ } \\
\text { multifoca } \\
1 \\
\text { seizures, } \\
\text { eye } \\
\text { deviation } \\
\text {, tonic } \\
\text { posturing } \\
\text { episodes } \\
\text { of status } \\
\text { epileptic } \\
\text { us/severe }\end{array}$ & $\begin{array}{c}\text { 3y/gener } \\
\text { alised } \\
\text { tonic } \\
\text { during } \\
\text { sleep/mo } \\
\text { derately } \\
\text { severe }\end{array}$ & $\begin{array}{c}\text { No } \\
\text { seizures }\end{array}$ & $\begin{array}{c}1 \mathrm{~m} / \\
\text { hypotoni } \\
\text { a and } \\
\text { cyanosis; } \\
\text { 3y/absen } \\
\text { ce with } \\
\text { eyelid } \\
\text { myoclon } \\
\text { ia/severe }\end{array}$ & $\begin{array}{c}\text { At } \\
\text { birth/neo } \\
\text { natal } \\
\text { seizures } \\
\text { with } \\
\text { apnea, } \\
\text { cyanosis } \\
\text { and } \\
\text { hyperton } \\
\text { us, } \\
\text { evolving } \\
\text { to status } \\
\text { epileptic } \\
\text { us/severe }\end{array}$ & $\begin{array}{c}2 \mathrm{w} / \text { seve } \\
\text { re } \\
\text { epilepsy } \\
\text { (semiolo } \\
\text { gy } \\
\text { unspecif } \\
\text { ied) }\end{array}$ \\
\hline
\end{tabular}




\begin{tabular}{|c|c|c|c|c|c|c|c|c|c|}
\hline & \multicolumn{9}{|c|}{$A T P 1 A 3$, heterozygous } \\
\hline $\begin{array}{l}\text { Patient } \\
\text { Nr/sex }\end{array}$ & $10 / M$ & $11 / F$ & $12 / \mathrm{M}$ & $13 / M$ & 14/M & $15 / F$ & $16 / M$ & $17 / F$ & $18 / F$ \\
\hline $\begin{array}{c}\text { Interictal } \\
\text { EEG }\end{array}$ & $\begin{array}{c}\text { Slow } \\
\text { backgrou } \\
\text { nd, } \\
\text { multifoca } \\
1 \\
\text { discharge } \\
\text { s }\end{array}$ & $\begin{array}{c}\text { Burst } \\
\text { suppress } \\
\text { ion and } \\
\text { multifoc } \\
\text { al } \\
\text { discharg } \\
\text { es }\end{array}$ & $\begin{array}{l}\text { Multifoc } \\
\text { al } \\
\text { discharg } \\
\text { es }\end{array}$ & $\begin{array}{c}\text { Multifoc } \\
\text { al } \\
\text { discharge } \\
\text { s }\end{array}$ & $\begin{array}{l}\text { Backgro } \\
\text { und with } \\
\text { beta } \\
\text { activity, } \\
\text { multifoc } \\
\text { al } \\
\text { discharg } \\
\text { es }\end{array}$ & $\begin{array}{c}\text { Right } \\
\text { temporal } \\
\text { slow and } \\
\text { sharp } \\
\text { wave } \\
\text { activity }\end{array}$ & $\begin{array}{c}\text { Focal } \\
\text { and } \\
\text { generalis } \\
\text { ed } \\
\text { discharg } \\
\text { es, photo } \\
\text { paroxys } \\
\text { mal } \\
\text { response }\end{array}$ & $\begin{array}{l}\text { Multifoc } \\
\text { al } \\
\text { discharg } \\
\text { es }\end{array}$ & $\begin{array}{c}\text { EEG } \\
\text { details } \\
\text { missing }\end{array}$ \\
\hline $\begin{array}{c}\text { Brain } \\
\text { MRI: } \\
\text { age/findin } \\
\text { gs }\end{array}$ & $\begin{array}{l}\text { 13y/mild } \\
\text { bilateral } \\
\text { frontote } \\
\text { mporal } \\
\text { atrophy }\end{array}$ & $\begin{array}{c}2 \mathrm{~m} / \text { diffu } \\
\text { se } \\
\text { polymicr } \\
\text { ogyria, } \\
\text { dilated } \\
\text { ventricle } \\
\text { s }\end{array}$ & $\begin{array}{c}\text { 8y/norm } \\
\text { al }\end{array}$ & $\begin{array}{l}\text { 1d/extens } \\
\text { ive, } \\
\text { bilateral, } \\
\text { perisylvi } \\
\text { an } \\
\text { predomin } \\
\text { ant, } \\
\text { polymicr } \\
\text { ogyria }\end{array}$ & $\begin{array}{c}\text { 1y and } \\
\text { 3y/bilate } \\
\text { ral } \\
\text { extensive } \\
\text { perisylvi } \\
\text { an } \\
\text { predomi } \\
\text { nant } \\
\text { polymicr } \\
\text { ogyria, } \\
\text { thick } \\
\text { corpus } \\
\text { callosum }\end{array}$ & $\begin{array}{c}30 y / \\
\text { right } \\
\text { perisylvi } \\
\text { an } \\
\text { polymicr } \\
\text { ogyria, } \\
\text { thick } \\
\text { corpus } \\
\text { callosum }\end{array}$ & $\begin{array}{c}3 \mathrm{~m}, 1 \mathrm{y}, \\
5 \mathrm{y}, 8 \mathrm{y}, \\
17 \mathrm{y} \text { and } \\
25 \mathrm{y} / \mathrm{bilat} \\
\text { eral } \\
\text { extensiv } \\
\mathrm{e} \\
\text { perisylvi } \\
\text { an } \\
\text { predomi } \\
\text { nant } \\
\text { polymicr } \\
\text { ogyria; } \\
\text { no signs } \\
\text { of } \\
\text { progressi } \\
\text { on }\end{array}$ & $\begin{array}{c}2 \mathrm{~d} / \\
\text { bilateral } \\
\text { extensiv } \\
\mathrm{e}, \\
\text { perisylvi } \\
\text { an } \\
\text { predomi } \\
\text { nant } \\
\text { polymicr } \\
\text { ogyria }\end{array}$ & $\begin{array}{c}2 \mathrm{~m} / \text { nor } \\
\mathrm{mal}\end{array}$ \\
\hline $\begin{array}{c}\text { Microceph } \\
\text { aly (OFC } \\
<-2 \text { SD) }\end{array}$ & No & $\begin{array}{c}\text { Yes, } \\
\text { congenit } \\
\text { al } \\
(-3 \mathrm{SD})\end{array}$ & No & $\begin{array}{c}\text { Yes, } \\
\text { postnatal } \\
(-2 \mathrm{SD})\end{array}$ & No & No & No & $\begin{array}{c}\text { Yes, } \\
\text { congenit } \\
\text { al (SD } \\
\text { not } \\
\text { available } \\
\text { ) }\end{array}$ & No \\
\hline $\begin{array}{c}\text { Other } \\
\text { Neurologi } \\
\text { cal } \\
\text { Features }\end{array}$ & $\begin{array}{l}\text { Profound } \\
\text { develop } \\
\text { mental } \\
\text { delay, } \\
\text { hypotoni } \\
\text { c- } \\
\text { dyskineti } \\
\text { c } \\
\text { quadripar } \\
\text { esis, } \\
\text { optic } \\
\text { atrophy }\end{array}$ & $\begin{array}{c}\text { Severe } \\
\text { develop } \\
\text { mental } \\
\text { delay, } \\
\text { hypotoni } \\
\text { c } \\
\text { quadripa } \\
\text { resis, } \\
\text { absent } \\
\text { ABR }\end{array}$ & $\begin{array}{c}\text { Severe } \\
\text { develop } \\
\text { mental } \\
\text { delay, } \\
\text { hypotoni } \\
\text { c } \\
\text { quadripa } \\
\text { resis }\end{array}$ & $\begin{array}{c}\text { Severe } \\
\text { develop } \\
\text { mental } \\
\text { delay, } \\
\text { severe } \\
\text { axial } \\
\text { hypotoni } \\
\text { a }\end{array}$ & $\begin{array}{c}\text { Severe } \\
\text { develop } \\
\text { mental } \\
\text { delay, } \\
\text { hypotoni } \\
\text { c } \\
\text { quadripa } \\
\text { resis, } \\
\text { central } \\
\text { apnoea }\end{array}$ & $\begin{array}{c}\text { Mild } \\
\text { intellectu } \\
\text { al } \\
\text { disability } \\
\text { and } \\
\text { dyspraxi } \\
\text { a, } \\
\text { migraine } \\
\text { with } \\
\text { somatose } \\
\text { nsory } \\
\text { aura }\end{array}$ & $\begin{array}{c}\text { Severe } \\
\text { intellectu } \\
\text { al } \\
\text { disability } \\
\text { d } \\
\text { dyskines } \\
\text { ia; } \\
\text { alternati } \\
\text { ng } \\
\text { hemipleg } \\
\text { ia } \\
\text { attacks } \\
\text { since age } \\
\text { 5m with } \\
\text { good } \\
\text { response } \\
\text { to } \\
\text { flunarizi } \\
\text { ne }\end{array}$ & $\begin{array}{l}\text { Irritabilit } \\
\text { y, limb } \\
\text { hyperton } \\
\text { us, } \\
\text { abnorma } \\
1 \\
\text { neonatal } \\
\text { reflexes }\end{array}$ & $\begin{array}{c}\text { Severe } \\
\text { develop } \\
\text { mental } \\
\text { delay, } \\
\text { dyskinet } \\
\text { ic } \\
\text { quadripa } \\
\text { resis, } \\
\text { central } \\
\text { apnoea }\end{array}$ \\
\hline
\end{tabular}




\begin{tabular}{|c|c|c|c|c|c|c|c|c|c|}
\hline & \multicolumn{9}{|c|}{$A T P 1 A 3$, heterozygous } \\
\hline $\begin{array}{l}\text { Patient } \\
\text { Nr/sex }\end{array}$ & $10 / M$ & $11 / F$ & $12 / \mathbf{M}$ & $13 / \mathrm{M}$ & $14 / M$ & $15 / F$ & $16 / \mathrm{M}$ & $17 / F$ & $18 / \mathbf{F}$ \\
\hline $\begin{array}{l}\text { Additional } \\
\text { Clinical } \\
\text { findings } \\
\text { and ECG }\end{array}$ & $\begin{array}{l}\text { Drug- } \\
\text { induced } \\
\text { (valproat } \\
\text { e) renal } \\
\text { Fanconi } \\
\text { syndrom } \\
\text { e; ECG: } \\
\text { normal }\end{array}$ & $\begin{array}{l}\text { Pierre- } \\
\text { Robin } \\
\text { sequenc } \\
\text { e; ECG: } \\
\text { normal }\end{array}$ & $\begin{array}{c}\text { No; } \\
\text { ECG: } \\
\text { normal }\end{array}$ & $\begin{array}{l}\text { Short QT } \\
\text { interval, } \\
\text { unusual } \\
\text { ST } \\
\text { segment } \\
\text { in } 12 \\
\text { lead } \\
\text { ECG, } \\
\text { with two } \\
\text { normal } \\
\text { Holter } \\
\text { ECGs }\end{array}$ & $\begin{array}{l}\text { Hypogen } \\
\text { etic right } \\
\text { lung } \\
\text { with } \\
\text { mild } \\
\text { right } \\
\text { ventricul } \\
\text { ar } \\
\text { hypertro } \\
\text { phy and } \\
\text { stomach } \\
\text { malrotati } \\
\text { on; } \\
\text { ECG: } \\
\text { normal }\end{array}$ & $\begin{array}{c}\text { No; ECG } \\
\text { unavaila } \\
\text { ble }\end{array}$ & $\begin{array}{c}\text { No; } \\
\text { ECG: } \\
\text { normal }\end{array}$ & $\begin{array}{l}\text { High } \\
\text { pitched } \\
\text { cry; } \\
\text { ECG: } \\
\text { normal }\end{array}$ & $\begin{array}{l}\text { No; } \\
\text { ECG: } \\
\text { normal }\end{array}$ \\
\hline $\begin{array}{l}\text { Novel/Ref. } \\
\text { (for } \\
\text { previously } \\
\text { reported } \\
\text { mutations) }\end{array}$ & $\begin{array}{l}\text { (Marzin } \\
\text { et al., } \\
\text { 2018) }\end{array}$ & Novel & Novel & $\begin{array}{c}\text { (Rosewic } \\
\text { h et al., } \\
\text { 2012) }\end{array}$ & Novel & Novel & $\begin{array}{c}\text { (Panagio } \\
\text { takaki et } \\
\text { al., } \\
\text { 2015) }\end{array}$ & Novel & Novel \\
\hline
\end{tabular}


Table 1 (continues)

\begin{tabular}{|c|c|c|c|c|c|}
\hline & \multicolumn{4}{|c|}{$A T P 1 A 3$, heterozygous } & ATP1A2, \\
\hline Patient $\mathrm{Nr} / \mathrm{sex}$ & 19/M & $20 / F$ & $21 / F$ & $22 / F$ & $23 / F$ \\
\hline $\begin{array}{c}\text { Mutation } \\
\text { (protein)/abbrevi } \\
\text { ation }\end{array}$ & $\begin{array}{l}\text { p.(Asp992dup)/ } \\
\text { A3-D992dup }\end{array}$ & $\begin{array}{l}\text { p.(Ser361Pro)** } \\
\text { /A3-S361P }\end{array}$ & $\begin{array}{c}\text { p. }(\text { Phe } 857 \mathrm{del}) / \\
\text { A3-F857del }\end{array}$ & $\begin{array}{l}\text { p.(Leu924Pro)/ } \\
\text { A3-L924P }\end{array}$ & $\begin{array}{l}\text { p.(Arg279Glyfs*4) } \\
\text { /A2-R279Gfs*4 }\end{array}$ \\
\hline $\begin{array}{l}\text { Inherited/De } \\
\text { novo/type of } \\
\text { analysis }\end{array}$ & De novo/WES & De novo/WES & $\begin{array}{c}\text { De novo } \\
\text { (mosaic)/WES }\end{array}$ & De novo/WES & $\begin{array}{c}\text { Inherited } \\
\text { (biparental)/WES }\end{array}$ \\
\hline $\begin{array}{l}\text { Age at last } \\
\text { evaluation }\end{array}$ & $\begin{array}{l}3 \mathrm{w} \text {, deceased } \\
\text { (progressive } \\
\text { worsening of } \\
\text { the general } \\
\text { conditions } \\
\text { during status } \\
\text { epilepticus) }\end{array}$ & $12 \mathrm{~m}$ & $18 \mathrm{y}$ & $4 y 7 m$ & $\begin{array}{l}1 \mathrm{~d}, \text { deceased } \\
\text { (progressive } \\
\text { respiratory } \\
\text { insufficiency) }\end{array}$ \\
\hline $\begin{array}{c}\text { Prenatal/perinata } \\
\text { I findings }\end{array}$ & Unremarkable & Unremarkable & Unremarkable & $\begin{array}{l}\text { Delivery } \\
\text { complicated by } \\
\text { meconium } \\
\text { aspiration }\end{array}$ & \begin{tabular}{|} 
First trimester: \\
increased nuchal \\
translucency; \\
second trimester: \\
polyhydramnios, \\
IUGR, fetal \\
hydrops, \\
microcephaly, \\
clenched hands, \\
and abnormal fetal \\
movements \\
consistent with \\
seizure activity \\
\end{tabular} \\
\hline $\begin{array}{c}\text { Age at seizure } \\
\text { onset/ } \\
\text { type/severity }\end{array}$ & $\begin{array}{c}\text { At birth/focal } \\
\text { motor with } \\
\text { tonic posturing, } \\
\text { evolving to } \\
\text { status } \\
\text { epilepticus/seve } \\
\text { re }\end{array}$ & $\begin{array}{l}3 \mathrm{~m} / \text { focal clonic, } \\
\text { bilateral tonic } \\
\text { posturing with } \\
\text { apnea/controlled } \\
\text { since age } 5 \mathrm{~m}\end{array}$ & $\begin{array}{c}\text { Day } \\
\text { 1/hiccoughs, } \\
\text { stiffening and } \\
\text { abducting; } \\
\text { 2m/left- focal; } \\
\text { 2y/ tonic, oral } \\
\text { automatisms, } \\
\text { apnea, severe } \\
\text { clusters } \\
\text { 30/day, often } \\
\text { evolving into } \\
\text { spasms; } \\
\text { 5y/tonic, } \\
\text { atonic, } \\
\text { migrating } \\
\text { focal seizures, } \\
\text { focal impaired } \\
\text { awareness; } \\
\text { 19y/focal, } \\
\text { tonic, } \\
\text { spasms/severe }\end{array}$ & $\begin{array}{c}\text { 6w/ multifocal } \\
\text { seizures; } \\
\text { 4y/epileptic } \\
\text { spasms. For } \\
\text { most of life } \\
\text { daily use of } \\
\text { emergency } \\
\text { antiseizure } \\
\text { medications } \\
\text { and bag+mask } \\
\text { ventilation } \\
\text { because of } \\
\text { compromising } \\
\text { focal seizures } \\
\text { or focal status } \\
\text { epilepticus/sev } \\
\text { ere }\end{array}$ & $\begin{array}{l}\text { At birth/neonatal } \\
\text { seizures }\end{array}$ \\
\hline
\end{tabular}




\begin{tabular}{|c|c|c|c|c|c|}
\hline & \multicolumn{4}{|c|}{$A T P 1 A 3$, heterozygous } & $\begin{array}{c}A T P 1 A 2, \\
\text { homozygous }\end{array}$ \\
\hline Patient $\mathrm{Nr} / \mathrm{sex}$ & $19 / \mathrm{M}$ & $20 / \mathbf{F}$ & $21 / F$ & $22 / F$ & $23 / F$ \\
\hline Interictal EEG & NA & $\begin{array}{c}3-7 \mathrm{~m} / \mathrm{slow} \\
\text { background, } \\
\text { more prominent } \\
\text { on the right }\end{array}$ & $\begin{array}{c}\text { 8y/poorly } \\
\text { organised } \\
\text { background, } \\
\text { bilateral } \\
\text { asynchronous } \\
\text { epileptiform } \\
\text { discharges; } \\
18 \mathrm{y} / \\
\text { multifocal } \\
\text { discharges } \\
\end{array}$ & $\begin{array}{c}\text { 10w/normal } \\
\text { background, } \\
\text { multifocal } \\
\text { discharges; 1- } \\
\text { 4y/slow } \\
\text { background } \\
\text { multifocal } \\
\text { discharges }\end{array}$ & NA \\
\hline $\begin{array}{l}\text { Brain MRI: } \\
\text { age/findings }\end{array}$ & $\begin{array}{l}\text { 1d/abnormality } \\
\text { of thalamus } \\
\text { morphology, } \\
\text { bilateral, } \\
\text { polymicrogyria } \\
\text { more severe } \\
\text { anteriorly }\end{array}$ & $\begin{array}{c}3 \mathrm{~m} / \text { bilateral } \\
\text { perisylvian } \\
\text { polymicrogyria }\end{array}$ & $\begin{array}{c}5 y / \text { mild } \\
\text { atrophy with } \\
\text { small right } \\
\text { hippocampus; } \\
\text { bilateral } \\
\text { anterior } \\
\text { delayed } \\
\text { myelination }\end{array}$ & $2 \mathrm{~m} /$ normal & $\begin{array}{l}\text { MRI not available; } \\
\text { Cranial CT: scan } \\
\text { ventriculomegaly, } \\
\text { diffuse } \\
\text { calcifications and } \\
\text { poor gyral } \\
\text { formation }\end{array}$ \\
\hline $\begin{array}{l}\text { Microcephaly } \\
\text { (OFC <-2SD) }\end{array}$ & $\begin{array}{l}\text { Yes, postnatal } \\
\text { (SD not } \\
\text { available) } \\
\end{array}$ & $\begin{array}{l}\text { Yes, congenital } \\
\quad(-3.2 \mathrm{SD})\end{array}$ & No & $\begin{array}{l}\text { Yes, postnatal } \\
\quad(-2.6 \mathrm{SD})\end{array}$ & Yes, congenital \\
\hline $\begin{array}{c}\text { Other } \\
\text { Neurological } \\
\text { Features }\end{array}$ & $\begin{array}{c}\text { Hypotonic } \\
\text { quadriparesis }\end{array}$ & $\begin{array}{l}\text { Axial hypotonia, } \\
\text { quadriparesis; } \\
\text { alternating } \\
\text { hemiplegia } \\
\text { attacks since age } \\
7 \mathrm{~m} \text {, flunarizine } \\
\text { started at } 12 \mathrm{~m}\end{array}$ & $\begin{array}{c}\text { Severe } \\
\text { intellectual } \\
\text { disability with } \\
\text { regression: } \\
\text { babbled at } \\
12 \mathrm{~m} \text { and } \\
\text { ceased at } 5 \mathrm{y}, \\
\text { walked at } 4 \mathrm{y} \\
\text { but became } \\
\text { unstable; } \\
\text { reluctant to } \\
\text { walk and non- } \\
\text { verbal at } 18 \mathrm{y} \\
\end{array}$ & $\begin{array}{c}\text { Severe- } \\
\text { profound } \\
\text { developmental } \\
\text { delay, not able } \\
\text { to grasp, } \\
\text { inconsistent } \\
\text { visual } \\
\text { attention, no } \\
\text { head control, } \\
\text { no oral feeding }\end{array}$ & $\begin{array}{l}\text { No spontaneous } \\
\text { respiratory drive; } \\
\text { generalised four } \\
\text { limb contractures }\end{array}$ \\
\hline $\begin{array}{c}\text { Additional } \\
\text { Clinical findings } \\
\text { and ECG }\end{array}$ & $\begin{array}{l}\text { No; ECG: } \\
\text { normal }\end{array}$ & $\begin{array}{l}\text { Congenital hip } \\
\text { dysplasia; ECG: } \\
\text { normal }\end{array}$ & $\begin{array}{c}\text { Constipation, } \\
\text { PEG, } \\
\text { fundoplication, } \\
\text { precocious } \\
\text { puberty, mild } \\
\text { scoliosis, } \\
\text { divergent } \\
\text { strabismus; } \\
\text { ECG: normal } \\
\end{array}$ & $\begin{array}{c}\text { Gastrostomy } \\
\text { fed, } \\
\text { constipation, } \\
\text { sialorrhea, } \\
\text { osteopenia } \\
\text { with pathologic } \\
\text { fracture, hip } \\
\text { dysplasia; } \\
\text { ECG: normal } \\
\end{array}$ & $\begin{array}{l}\text { Hypertelorism, } \\
\text { proptotic eyes with } \\
\text { shallow orbits, } \\
\text { periorbital edema, } \\
\text { high arched palate } \\
\text { and a short neck }\end{array}$ \\
\hline $\begin{array}{c}\text { Novel/Ref. (for } \\
\text { previously } \\
\text { reported } \\
\text { mutations) }\end{array}$ & Novel & Novel & $\begin{array}{c}\text { (Retterer et al., } \\
\text { 2016) }\end{array}$ & $\begin{array}{l}\text { (Arystarkhova } \\
\text { et al., 2019) }\end{array}$ & $\begin{array}{c}\text { (Monteiro et al., } \\
2020)\end{array}$ \\
\hline
\end{tabular}


Table 1 - Clinical features in our cohort. Patients 1-19 are ordered by c.DNA. The position of Patients 20-22 at the end of the table reflects their inclusion in this series at a late stage. ${ }^{\text {FFor }}$ deceased patients, the cause of death is reported in brackets. The c.DNA positions refer to the reference transcripts NM_000702.4 (for ATP1A2) and NM_152296.5 (for ATP1A3). Abbreviations and symbols: **, recurrent mutation identified in $>1$ unrelated patients from this cohort; d, days; w, weeks; m, months; y, years; ABR, auditory brainstem response; ADHD, attention deficit hyperactivity disorder; ID, intellectual disability; IPS, intermittent photic stimulation; IUGR, intrauterine growth restriction; NA, not available/not applicable; PEG, percutaneous endoscopic gastrostomy; OFC, occipital frontal circumference; TC, tonic-clonic; TGP, targeted-gene panel; US, ultrasound; WES, whole-exome sequencing; WGS, whole-genome sequencing. 
Table 2

\begin{tabular}{|c|c|c|c|c|c|c|c|c|c|c|c|c|c|c|c|c|c|}
\hline \multicolumn{3}{|c|}{ Patient/Mutation } & \multicolumn{5}{|c|}{ Phenotype } & \multicolumn{10}{|c|}{ Location/Function } \\
\hline $\operatorname{Pt} N$ & Gene & Variant & $\begin{array}{l}\text { Died } \\
0-3 y\end{array}$ & DD/ID & MIC (subtype) & PMG & $\mathbf{A H}$ & $\begin{array}{l}\text { Struct } \\
\text { ural } \\
\text { locati } \\
\text { on }\end{array}$ & $\begin{array}{c}\text { Cell } \\
\text { surviva } \\
\text { l in } \\
\text { culture } \\
\text { (under } \\
\text { ouabai } \\
\text { n } \\
\text { selectio } \\
\text { n) }\end{array}$ & $\begin{array}{c}\text { Phosph } \\
\text { o- } \\
\text { rylatio } \\
\text { n }\end{array}$ & $\begin{array}{c}\text { Ma } \\
\mathbf{x} \\
\text { TO } \\
\text { rat } \\
\text { e }\end{array}$ & $\begin{array}{c}\text { NKA } \\
\text { activity/ } \\
\text { mg } \\
\text { membra } \\
\text { ne } \\
\text { protein }\end{array}$ & $\begin{array}{c}\mathrm{Na}^{+} \\
\text {affinity }\end{array}$ & $\begin{array}{l}\mathrm{Na}^{+} / \mathbf{K}^{+} \\
\text {selectiv } \\
\quad \text { ity }\end{array}$ & $\underset{\text { affinity }}{\mathbf{K}^{+}}$ & $\begin{array}{c}\text { Conformati } \\
\text { onal change } \\
\text { E1-E2 }\end{array}$ & $\begin{array}{c}\text { Conformatio } \\
\text { nal change } \\
\text { E1P-E2P }\end{array}$ \\
\hline \multicolumn{18}{|c|}{ Group 1 - Variant does not support cell survival in culture (this study; $N=9$ mutations in 11 patients) } \\
\hline 2 & $\begin{array}{c}\text { ATP1 } \\
\text { A2 } \\
\end{array}$ & $\begin{array}{c}\mathrm{A} 2- \\
\mathrm{C} 341 \mathrm{~F} \\
\end{array}$ & No & SEV & No & No & No & M4 & No & $10-15 \%$ &.. &. &.. &.. &.. &.. &.. \\
\hline 3 & $\begin{array}{c}\text { ATP1 } \\
\text { A2 } \\
\end{array}$ & $\begin{array}{c}\text { A2- } \\
\text { G366A }\end{array}$ & Yes & SEV & No & No & No & $\mathrm{P}$ & No & $61 \%$ &.. &.. & $\begin{array}{l}\downarrow 3-5 \\
\text { fold } \\
\end{array}$ &.. & $\begin{array}{l}\downarrow>15 \\
\text { fold } \\
\end{array}$ &.. & $\begin{array}{c}\text { shift } \\
\text { E1P } \rightarrow \rightarrow \text { E2P }\end{array}$ \\
\hline 4 & $\begin{array}{c}\mathbf{A T P 1} \\
\mathbf{A 2}\end{array}$ & $\begin{array}{c}\text { A2- } \\
\text { G366A }\end{array}$ & Yes & SEV & $\begin{array}{c}\text { MIC } \\
\text { (postnatal)* }\end{array}$ & No & No & $\mathrm{P}$ & No & $61 \%$ &.. &.. & $\begin{array}{l}\downarrow 3-5 \\
\text { fold }\end{array}$ &.. & $\begin{array}{l}\downarrow>15 \\
\text { fold }\end{array}$ &.. & $\begin{array}{c}\text { shift } \\
\text { E1P } \rightarrow \rightarrow \text { E2P }\end{array}$ \\
\hline 7 & $\begin{array}{c}\text { ATP1 } \\
\text { A3 } \\
\end{array}$ & $\begin{array}{c}\text { A3- } \\
\text { L292R } \\
\end{array}$ & Yes & SEV & $\begin{array}{c}\text { MIC } \\
\text { (postnatal)** }\end{array}$ & Yes & No & M3 & No & $61 \%$ &.. &.. & $\begin{array}{l}\downarrow 3-5 \\
\text { fold } \\
\end{array}$ &.. & $\begin{array}{l}\downarrow>15 \\
\text { fold } \\
\end{array}$ &.. &.. \\
\hline 8 & $\begin{array}{c}\text { ATP1 } \\
\text { A3 }\end{array}$ & $\begin{array}{c}\text { A3- } \\
\text { G316V }\end{array}$ & No & SEV & No & No & No & M4 & No & $70 \%$ & .. &.. & $\uparrow 4$ fold &.. & $\begin{array}{l}\downarrow>15 \\
\text { fold }\end{array}$ &. &.. \\
\hline 9 & $\begin{array}{c}\text { ATP1 } \\
\text { A3 } \\
\end{array}$ & A3-S361P & No & MOD & $\begin{array}{c}\text { MIC } \\
\text { (postnatal)** }\end{array}$ & No & No & $\mathrm{P}$ & No & $10-15 \%$ &. & .. &.. &.. &.. &.. &.. \\
\hline 20 & $\begin{array}{c}\text { ATP1 } \\
\text { A3 }\end{array}$ & A3-S361P & No & NA & $\begin{array}{c}\text { MIC } \\
\text { (congenital)** }\end{array}$ & Yes & Yes & $\mathrm{P}$ & No & $10-15 \%$ &.. &. &.. &. &.. &.. &. \\
\hline 11 & $\begin{array}{c}\text { ATP1 } \\
\text { A3 } \\
\end{array}$ & $\begin{array}{c}\text { A3- } \\
\text { K764del } \\
\end{array}$ & Yes & SEV & $\begin{array}{c}\text { MIC } \\
\text { (congenital)** }\end{array}$ & Yes & No & M5 & No & $10-15 \%$ &.. &.. &.. &.. &.. &.. &.. \\
\hline 12 & $\begin{array}{c}\text { ATP1 } \\
\text { A3 } \\
\end{array}$ & $\begin{array}{c}\text { A3- } \\
\text { P775R } \\
\end{array}$ & No & SEV & No & No & No & M5 & No & $\sim 0 \%$ &.. &.. &.. &.. &.. &.. &.. \\
\hline 16 & $\begin{array}{c}\text { ATP1 } \\
\text { A3 }\end{array}$ & $\begin{array}{c}\text { A3- } \\
\text { L888P } \\
\end{array}$ & No & SEV & No & Yes & Yes & L7-8 & No & $\sim 0 \%$ & .. &.. &.. &.. &.. &.. &.. \\
\hline \multirow[t]{2}{*}{19} & $\begin{array}{c}\text { ATP1 } \\
\text { A3 } \\
\end{array}$ & $\begin{array}{c}\text { A3- } \\
\text { D992dup }\end{array}$ & Yes & NA & $\begin{array}{c}\text { MIC } \\
\text { (postnatal) }\end{array}$ & Yes & No & M10 & No & $\sim 0 \%$ &.. &.. &.. &.. &.. &.. &.. \\
\hline & & & $\begin{array}{c}5 \text { of } \\
11 \\
\end{array}$ & $\begin{array}{l}8 \text { of } 9 \\
\text { SEV }\end{array}$ & 6 of 11 & $\begin{array}{c}5 \text { of } \\
11 \\
\end{array}$ & $\begin{array}{c}2 \text { of } \\
11\end{array}$ & & & & & & & & & & \\
\hline \multicolumn{18}{|c|}{ Group 2 - Variant does support cell survival in culture (this study; $N=5$ mutations in 6 patients) } \\
\hline 1 & $\begin{array}{c}\text { ATP1 } \\
\text { A2 }\end{array}$ & $\begin{array}{c}\mathrm{A} 2- \\
\mathrm{I} 293 \mathrm{M}\end{array}$ & No & MOD & No & No & No & M3 & Yes & $>50 \%$ & $\begin{array}{l}27 \\
\% \\
\end{array}$ & $\sim 17 \%$ & $\begin{array}{l}\downarrow 3-5 \\
\text { fold }\end{array}$ & $\downarrow$ &.$\cdot$ & $\begin{array}{c}\text { shift } \\
\mathrm{E} 2 \rightarrow \rightarrow \mathrm{E} 1\end{array}$ & $\begin{array}{c}\text { shift } \\
\text { E2P } \rightarrow \rightarrow \text { E1P }\end{array}$ \\
\hline
\end{tabular}




\begin{tabular}{|c|c|c|c|c|c|c|c|c|c|c|c|c|c|c|c|c|c|}
\hline 5 & $\begin{array}{c}\text { ATP1 } \\
\text { A2 } \\
\end{array}$ & $\begin{array}{c}\text { A2- } \\
\text { R593Q } \\
\end{array}$ & No & No & No & No & No & $\mathrm{P}$ & Yes & $>50 \%$ & $\begin{array}{l}42 \\
\% \\
\end{array}$ & $\sim 36 \%$ & $\begin{array}{c}\sim \text { wild- } \\
\text { type }\end{array}$ & $\downarrow$ &.. & shift E2 $\rightarrow$ E1 & $\begin{array}{c}\text { shift } \\
\text { E2P } \rightarrow \text { E1P }\end{array}$ \\
\hline 6 & $\begin{array}{c}\text { ATP1 } \\
\text { A2 } \\
\end{array}$ & $\begin{array}{c}\text { A2- } \\
\text { R908Q }\end{array}$ & No & MOD & $\begin{array}{c}\text { MIC } \\
\text { (postnatal)** }\end{array}$ & Yes & No & L7-8 & Yes & $\sim 26 \%$ & $\begin{array}{l}84 \\
\% \\
\end{array}$ & $\sim 22 \%$ & $\begin{array}{l}\sim \text { wild- } \\
\text { type }\end{array}$ &.. &.. & shift E1 $\rightarrow$ E2 & .. \\
\hline 14 & $\begin{array}{c}\text { ATP1 } \\
\text { A3 }\end{array}$ & $\begin{array}{c}\text { A3- } \\
\text { D887Y }\end{array}$ & No & SEV & No & Yes & No & L7-8 & Yes & $\sim 35 \%$ & $\begin{array}{l}5 \\
\% \\
\end{array}$ & $\sim 33 \%$ & $\begin{array}{l}\text { 〜wild- } \\
\text { type }\end{array}$ & $\downarrow$ &.. & shift E2 $\rightarrow$ E1 &.. \\
\hline 15 & $\begin{array}{c}\text { ATP1 } \\
\text { A3 } \\
\end{array}$ & $\begin{array}{c}\text { A3- } \\
\text { D887Y } \\
\end{array}$ & No & MILD & No & Yes & No & L7-8 & Yes & $\sim 35 \%$ & $\begin{array}{l}95 \\
\% \\
\end{array}$ & $\sim 33 \%$ & $\begin{array}{l}\text { 〜wild- } \\
\text { type }\end{array}$ & $\downarrow$ &.. & shift E2 $\rightarrow \mathrm{E} 1$ &.. \\
\hline \multirow[t]{2}{*}{18} & $\begin{array}{c}\text { ATP1 } \\
\text { A3 }\end{array}$ & $\begin{array}{c}\text { A3- } \\
\text { P972del }\end{array}$ & Yes & SEV & No & No & No & L9-10 & Yes & $\sim 35 \%$ & $\begin{array}{l}85 \\
\%\end{array}$ & $\sim 30 \%$ & $\stackrel{\downarrow}{\downarrow}$ & $\uparrow$ &.. & shift E1 $\rightarrow \mathrm{E} 2$ & $\begin{array}{c}\text { shift } \\
\mathrm{E} 2 \mathrm{P} \rightarrow \mathrm{E} 1 \mathrm{P}\end{array}$ \\
\hline & & & $\begin{array}{c}1 \text { of } \\
6\end{array}$ & $\begin{array}{l}2 \text { of } 6 \\
\text { SEV }\end{array}$ & 1 of 6 & of 6 & 0 of 6 & & & & & & & & & & \\
\hline
\end{tabular}

Table 2 - Schematic representation of the main clinical features in our cohort with structural location and functional effect of the corresponding mutations. We separated the 17 patients (14 mutations) for whom experimental data were available from this study into two groups based on COS-1 cell survival under ouabain selection pressure. The mutations are distributed across the following locations: phosphorylation domain (P), transmembrane helices (M3-10) and their intervening loops (L). Nine different aspect of the NKA pump function investigated by at least one of the different experimental approaches are showed. Abbreviations and symbols: AH, alternating hemiplegia; DD/ID, developmental delay/intellectual disability; MIC, microcephaly $(*=-2 \mathrm{SD} ; * *=>-3 \mathrm{SD}$; no asterisk means there is microcephaly but SD are unknown); MOD, moderate; NA, not applicable/not available; PMG, polymicrogyria; Pt, patient; SEV, severe; TO, turnover. 


\section{Supplementary Materials}

\section{$A T P 1 A 2-$ and $A T P 1 A 3$-associated early profound epileptic encephalopathy and polymicrogyria}

Annalisa Vetro ${ }^{1}$, Hang N. Nielsen ${ }^{2}$, Rikke Holm ${ }^{2}$, Robert F. Hevner ${ }^{3}$, Elena Parrini ${ }^{1}$, Zoe Powis, $\mathrm{MS}^{4}$, Rikke S. Møller ${ }^{5,6}$, Cristina Bellan ${ }^{7}$, Alessandro Simonati ${ }^{8}$, Gaétan Lesca $^{9}$, Katherine L. Helbig $^{10}$, Elizabeth E. Palmer ${ }^{11,12}$, Davide Mei $^{1}$, Elisa Ballardini ${ }^{13}$, Arie Van Haeringen ${ }^{14}$, Steffen Syrbe ${ }^{15}$, Vincenzo Leuzzi ${ }^{16}$, Giovanni Cioni ${ }^{17}$, Cynthia J. Curry ${ }^{18}$, Gregory Costain ${ }^{19}$, Margherita Santucci ${ }^{20,21}$, Karen Chong ${ }^{22}$, Grazia M. S. Mancini ${ }^{23}$, Jill Clayton-Smith ${ }^{24}$, ATP1A2/A3-collaborators ${ }^{\S}$, Stefania Bigoni ${ }^{25}$, Ingrid E. Scheffer ${ }^{26}$, William B. Dobyns ${ }^{27 *}$, Bente Vilsen ${ }^{2 *}$, and Renzo Guerrini ${ }^{1 *}$ 


\section{Sections:}

Supplementary Materials and Methods .............................................................................................3

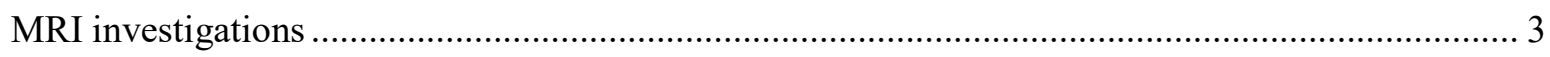

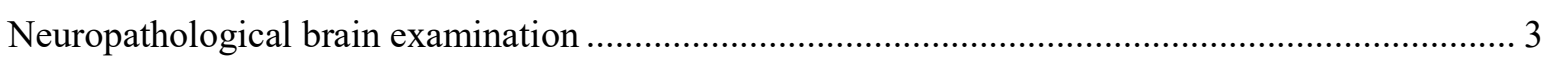

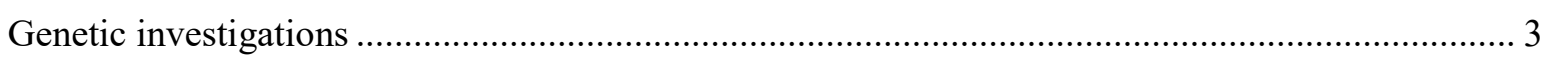

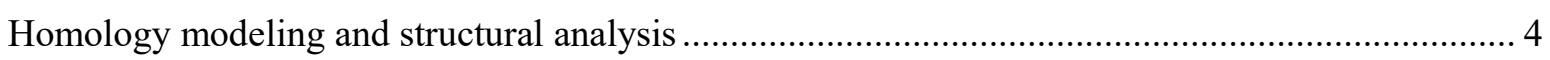

Functional characterization of ATP1A2-A3 heterozygous mutations .............................................. 4

Supplementary Results ....................................................................................................................................7

Functional characterization of ATP1A2-A3 heterozygous mutations - $\mathrm{Na}+$ - and $\mathrm{K}+$-binding

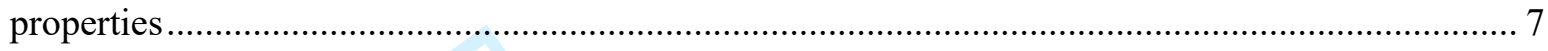

Functional characterization of $A T P 1 A 2-A 3$ heterozygous mutations - E1-E2 and E1P-E2P

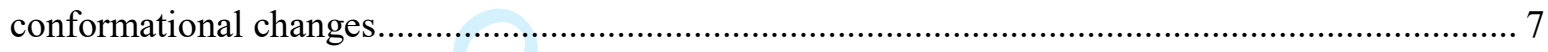

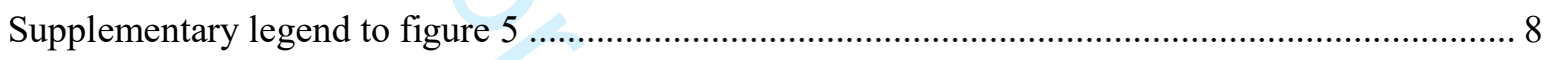

Supplementary Figures.....................................................................................................................................9

Supplementary Figure 1 - Brain MRI in two patients with progressive brain, brainstem and

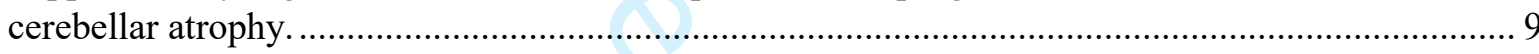

Supplementary Figure 2 - Multiple sequence alignment and conservation analysis ....................... 10

Supplementary Figure 3 Distribution of the ATP1A2 and ATP1A3 disease mutations on linear

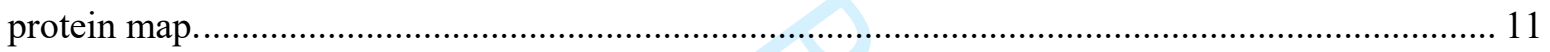

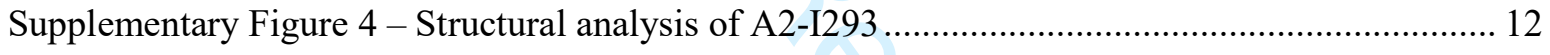

Supplementary Figure 5 - Structural analysis of A2-C341 and A3-L924 ....................................... 13

Supplementary Figure 6 - Structural analysis of A2-G366 and A3-S361 ...................................... 14

Supplementary Figure 7 - Structural analysis of A2-R593 and A3-D609 ...................................... 15

Supplementary Figure 8 - Structural analysis of A3-F857, A3-D887, A3-L888, A3-G893, and A2-

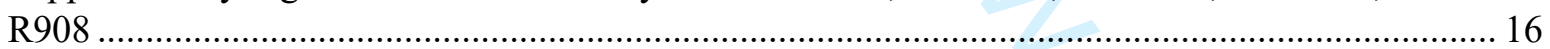

Supplementary Figure 9 - Structural analysis of A3-L292, A3-G316, A3-P775, and A3-D801...... 17

Supplementary Figure 10 - Structural analysis of A3-K764, A3-P972, and A3-D992 .................... 18

Supplementary Figure $11-\mathrm{K}+$ affinity determined from K+ dependence of NKA activity on stably

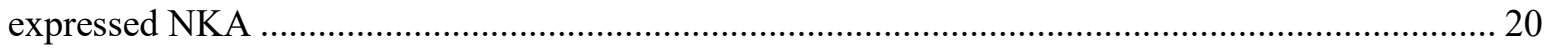

Supplementary Figure 12 - Shift of E1-E2 and E1P-E2P conformational equilibria ...................... 21

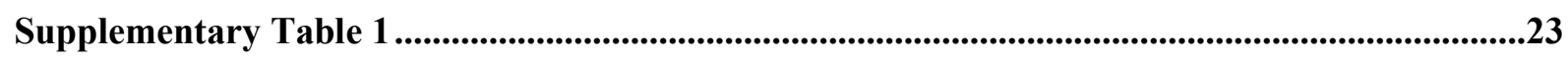

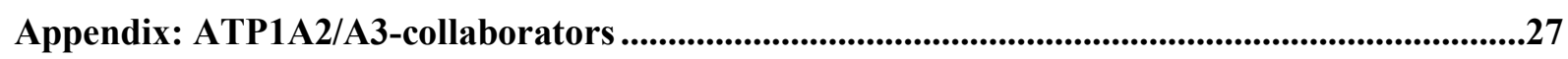

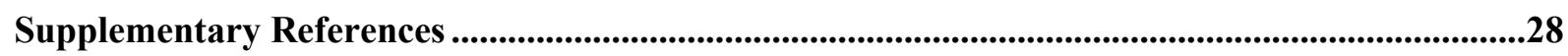




\section{Supplementary Materials and Methods}

\section{MRI investigations}

All patients underwent standard brain magnetic resonance imaging (MRI) as part of their routine clinical care. Data were acquired using 1.5-T or 3-T systems, with T1-weighted, T2-weighted, and fluid attenuated inversion recovery (FLAIR) sequences in axial, sagittal, and coronal planes at the respective centers. We used the term polymicrogyria to designate a developmental abnormality of the cerebral cortex characterized on MRI by an abnormal cortical folding, with an excessive number of abnormally small gyri, areas of infolding, variably associated with shallow sulci, thickened cortex and abnormal sulcation (Guerrini and Dobyns, 2014).

\section{Neuropathological brain examination}

We removed the brain of Patients 11 and 23 within 48 hours after death and fixed them in $70 \%$ ethanol (Patient 11 ) or $10 \%$ formalin (Patient 23 ) according to standard procedures. After macroscopic examination, we embedded representative samples of fixed brain in paraffin, sectioned at $6 \mu \mathrm{m}$, and mounted them on glass slides, using standard protocols for Hematoxylin and eosin (H-E), Luxol fast blue (LFB), and cresyl violet (Nissl) staining. We acquired brightfield images using a Carl Zeiss Axio Imager Z1 microscope equipped with motorized stage and MosaiX software (Zeiss, USA), photographed digitally images, and processed them using Photoshop (Adobe, San Jose, CA, USA) to optimize brightness, contrast, and resolution.

\section{Genetic investigations}

We performed trio-based whole exome sequencing (WES) in Patients 1, 3, 4, 6-22, and target-enrichment genepanel analysis (TGP) in Patients 2, and 5. Methods for TGP are reported in reference (Møller et al., 2015). For WES library preparation and target enrichment we used either the SureSelectXT Clinical Research Exome or the SureSelectXT Human All Exon v6 kit (Agilent Technologies, Santa Clara, CA). We sequenced the captured DNA libraries by a paired-end protocol on Illumina sequencers (HiSeq4000 or NextSeq550, Illumina, San Diego, CA, USA) to obtain an average coverage of above $80 x$, with $97.6 \%$ of target bases covered at least 10x. We performed bioinformatics analysis by standard procedures. Briefly, we aligned the sequencing reads to the GRCh37/hg19 human genome reference assembly by the BWA software package (Li and Durbin, 2009) and used the GATK suite for base quality score recalibration, realignment of insertion/deletions (InDels), and variant calling, according to GATK Best Practices recommendations (DePristo et al., 2011). For the annotation and filtering of exonic/splicesite single-nucleotide variants (SNVs) and coding InDels we used commercially available software (VarSeq, Golden Helix, Inc v1.4.6; Varvis, Limbus Medical Technologies), focusing on variants with minor allele frequency (MAF) lower than 0.01 in the GnomAD database (http://gnomad.broadinstitute.org/). We further excluded population-specific variants by interrogating our internal database (WES data from over 900 patients with DEE and 200 healthy parents) and evaluated the potential functional impact of SNVs and InDels by the pre-computed genomic variants score from dbNSFP (Liu et al., 2011), which was integrated in the annotation pipeline. We also 
manually interrogated in-silico prediction tools (Schwarz et al., 2010; Jagadeesh et al., 2016; Rentzsch et al., 2019), as well as evolutionary conservation scores (Cooper et al., 2005; Pollard et al., 2010). For selected variants, we visually inspected the quality of reads alignment by using the Integrative Genomics Viewer (Thorvaldsdóttir et al., 2013) and then proceeded to validation and segregation analysis by Sanger sequencing (primers and conditions available on request). Patient 7 was studied by whole-genome sequencing (WGS) as reported elsewhere (Palmer et al., 2018). The possible contribution of known MCD genes to the phenotype was excluded by interrogating NGS data generated by WGS and WES in all patients. Variants were classified according to the recommendation of the ACMG (American College of Medical Genetics and Genomics) (Richards et al., 2015), and reported following the guidelines of the Human Genome Variation Society (HGVS, http://www.hgvs.org/mutnomen). For cDNA numbering: nucleotide numbering uses +1 as the A of the ATG translation initiation codon in the reference sequence (Accession numbers: ATP1A2, NM_000702.4; ATP1A3, NM_152296.5). We submitted all novel variants to the publicly available database DECIPHER (http://decipher.sanger.ac.uk).

To calculate the relative percentage of mutations associated with DEE/PMG we searched the Human Gene Mutation Database (HGMD Professional, last accessed June 2020) for previously reported mutations in ATP1A2 and ATP1A3. We focused on heterozygous mutations classified as DM (disease causing mutations) by the HGMD curators (Stenson et al., 2017), and stratified them based on the reported phenotype.

\section{Homology modeling and structural analysis}

To show the evolutionary conservation of the mutated residues, we obtained the protein sequence alignment of human ATP1A2 and ATP1A3, their paralogs ATP1A1 and ATP1A4, and their orthologs in nine different species (Pan troglodytes, Rattus norvegicus, Sus scrofa, Canis familiaris, Gallus gallus, Xenopus laevis, Danio renio, Drosophila melanogaster, Caenorabditis elegans) by using Clustal Omega (Sievers et al., 2011). Because the atomic structures of the ATP1A2 and ATP1A3 isoforms of NKA have not been determined, the structural analysis of the mutations was based on the available high-resolution crystal structures of the $\alpha 1 \beta 1 \gamma$ protein complex in $\mathrm{Na}^{+}-$ bound E1 form (PDB ID 3WGV, chain A) (Kanai et al., 2013) and $\mathrm{K}^{+}$-bound E2 form (PDB ID 2ZXE) (Shinoda et al., 2009) as previously reported (Holm et al., 2016) using Pymol (Schrödinger, 2018).

\section{Functional characterization of $A T P 1 A 2 / A 3$ heterozygous mutations}

A number of assays have been developed to determine the function of mutant NKA pumps expressed in cell culture, including measurements of cell survival under ouabain selection, $\mathrm{Na}+$ and $\mathrm{K}+$ affinity and $\mathrm{Na}+-\mathrm{K}+$ specificity, expression level (active site concentration) and turnover rate, and distribution of the E1 and E2 states. We used several of these methods to investigate the functional consequences of 14 mutations (Table 2 and Supplementary Table 1) (Toustrup-Jensen et al., 2014; Nielsen et al., 2019; Roenn et al., 2019). We did not further characterize A3-D609Y, A3-D801N, A3-G893W, and A3-L924P as their impact is already well understood as mentioned above, and A3-F857del because Patient 21 was included in this series at a late stage.

Briefly, the mutations were introduced into full-length cDNA encoding ouabain-resistant human $\alpha 2-$ and $\alpha 3-$ isoforms, and the wild-type and mutant cDNA constructs were transfected into COS-1 cells. We examined whether the mutants could be stably expressed in the cells under ouabain selection pressure. In this method, the ouabain- 
sensitive endogenous NKA pump is silenced by adding ouabain to the COS-1 cell culture, and the survival of the cells therefore depends on the functional capability of the exogenous mutant enzyme, because $\mathrm{Na}^{+}$and $\mathrm{K}^{+}$transport is mandatory for cell viability. Hence, under these conditions stable cell lines expressing the exogenous NKA can be isolated if the exogenous enzyme retains at least 5-10\% of wild-type activity (Holm et al., 2016). Where absence of NKA pump activity precluded cell survival and thus the isolation of a stable cell line, we transiently expressed mutant and wild-type constructs by transfection of the COS-1 cells in the presence of siRNA to knock down the endogenous NKA as previously described (Nielsen et al., 2019). The siRNA used targeted the mRNA corresponding to part of the ouabain-binding site of the endogenous NKA, which is located in the extracellular loop between transmembrane helices M1 and M2. The presence of the correct mutation was verified by full-length sequencing of all the DNA constructs before transfection and by sequencing genomic DNA from the isolated stable cell line. Irrespective of whether mutants were stably or transiently expressed, we analysed the function of the expressed enzyme on plasma membranes isolated by differential centrifugation and made leaky by treatment with alamethicin to allow access of incubation media from both sides of the membrane. The NKA pump activity of the isolated stably transfected cell lines was studied on the isolated leaky plasma membranes at $37^{\circ} \mathrm{C}$ by following the liberation of Pi using the Baginski colorimetric method (Baginski et al., 1967). The medium contained $30 \mathrm{mM}$ histidine (pH 7.4), $1 \mathrm{mM}$ EGTA, $3 \mathrm{mM} \mathrm{MgCl}_{2}$, and $10 \mu \mathrm{M}$ ouabain to inhibit the endogenous COS-1 cell NKA, together with varying $\mathrm{Na}^{+}, \mathrm{K}^{+}$, and ATP concentrations as described below. For background subtraction, similar measurements were carried out in the presence of $10 \mathrm{mM}$ ouabain (inhibiting all NKA activity). For maximum NKA pump activity used to calculate the turnover rate, the additions were $130 \mathrm{mM} \mathrm{NaCl}, 20 \mathrm{mM} \mathrm{KCl}, 3 \mathrm{mM}$ ATP. For $\mathrm{K}^{+}$dependence of NKA pump activity, the additions were $40 \mathrm{mM} \mathrm{NaCl}, 3 \mathrm{mM} \mathrm{ATP}$, and varying concentrations of $\mathrm{KCl}$. For ATP dependence of NKA activity, the additions were $130 \mathrm{mM} \mathrm{NaCl}, 20 \mathrm{mM} \mathrm{KCl}$, and varying concentrations of ATP.

We performed phosphorylation studies with $\left[\gamma^{32} \mathrm{P}\right]$ ATP on the leaky membranes as previously described (Toustrup-Jensen et al., 2014; Nielsen et al., 2019; Roenn et al., 2019). For $\mathrm{Na}^{+}$dependence of phosphorylation, the reaction was carried out for $10 \mathrm{~s}$ at $0{ }^{\circ} \mathrm{C}$ with $2 \mu \mathrm{M}\left[\gamma^{32} \mathrm{P}\right] \mathrm{ATP}$ in $20 \mathrm{mM}$ Tris (pH 7.5), $3 \mathrm{mM} \mathrm{MgCl} 2,1 \mathrm{mM}$ EGTA, $20 \mu \mathrm{g}$ oligomycin/ml (to prevent dephosphorylation), ouabain to inhibit the endogenous COS-1 cell NKA, and varying concentrations of $\mathrm{Na}^{+}$(and $\mathrm{NMG}^{+}$to maintain constant ionic strength) with $\mathrm{Cl}^{-}$. The phosphorylation level determined under stoichiometric conditions, i.e. at a saturating $\mathrm{Na}^{+}$concentration of $150 \mathrm{mM}$, reflects the expression level ("active site concentration") and was used for calculating the turnover rate by relating the maximum NKA pump activity to the active site concentration. For $\mathrm{K}^{+}$dependence, phosphorylation was carried out for $10 \mathrm{~s}$ at $0{ }^{\circ} \mathrm{C}$ with $2 \mu \mathrm{M}\left[\gamma-{ }^{32} \mathrm{P}\right] \mathrm{ATP}$ in $20 \mathrm{mM}$ Tris (pH 7.5), $50 \mathrm{mM} \mathrm{NaCl}, 3 \mathrm{mM} \mathrm{MgCl}, 1 \mathrm{mM}$ EGTA, ouabain to inhibit the endogenous COS-1 cell NKA, and various concentrations of $\mathrm{KCl}$ (with choline chloride to maintain a constant ionic strength). In these experiments, oligomycin was absent to allow dephosphorylation. Shift of the distribution between the phosphoenzyme conformations E1P and E2P was determined by ADP-sensitivity of the phosphoenzyme. To this end, phosphorylation was carried out by incubation with $2 \mu \mathrm{M}\left[\gamma^{32}{ }^{32}\right] \mathrm{ATP}$ for $5 \mathrm{~s}$ at $0{ }^{\circ} \mathrm{C}$ in $20 \mathrm{mM}$ Tris (pH 7.5), $50 \mathrm{mM} \mathrm{NaCl}, 3 \mathrm{mM} \mathrm{MgCl}_{2}, 1 \mathrm{mM}$ EGTA, and ouabain to inhibit the endogenous enzyme. To induce dephosphorylation, $2.5 \mathrm{mM}$ ADP with $1 \mathrm{mM}$ ATP (non-radioactive) was added followed by termination of the reaction at various time intervals. In all phosphorylation experiments, the ouabain concentration added was $10 \mu \mathrm{M}$ for the stably expressed NKA and $100 \mu \mathrm{M}$ for the transiently expressed NKA to make sure that the endogenous enzyme (making up a larger fraction of the total amount of NKA in this case) did not contribute 
to the results. Phosphorylation was terminated by acid quenching, and the acid-precipitated ${ }^{32} \mathrm{P}-$ labelled phosphoenzyme was separated by SDS-polyacrylamide gel electrophoresis and quantified by phosphor imaging. We performed all experiments independently at least three times, using different preparations of isolated plasma membranes for each mutant and wild-type. Data points shown in figures are mean values with standard deviation (SD) indicated by error bars. The SigmaPlot program (SPSS, Inc.) was used to fit relevant equations to data points using non-linear regression as previously described (Nielsen et al., 2019). We determined the apparent affinities $\left(\mathrm{K}_{0.5}\right.$ values, ligand concentration giving half-maximum effect) Hill coefficients $\left(\mathrm{n}_{\mathrm{H}}\right)$ by fitting the Hill equation to each independent set of data points, followed by calculation of the mean values and SD shown in figure legends. The lines shown in the figures represent the best fit to all data points. 


\section{Supplementary Results}

\section{Functional characterization of $A T P 1 A 2-A 3$ heterozygous mutations - Na+- and K+-binding properties}

Phosphorylation of the NKA pump requires the binding of three $\mathrm{Na}^{+}$ions at the transport sites of the E1 form, which activates the transfer of the $\gamma$-phosphoryl group from ATP to NKA forming the E1P phosphorylated intermediate (cf. reaction cycle in Fig. 5D). To address the affinity for $\mathrm{Na}^{+}$, we examined the $\mathrm{Na}^{+}$dependence of phosphorylation in all the stably expressed mutants and the transiently expressed mutants showing robust phosphorylation (Fig. 6A). A marked 3- to 5-fold reduction of the apparent $\mathrm{Na}^{+}$affinity (increase of $\mathrm{K}_{0.5}$ ) was observed for A2-I293M, A2-G366A, and A3-L292R, whereas A3-G316V showed a 4-fold increase of apparent $\mathrm{Na}^{+}$affinity (decrease of $\mathrm{K}_{0.5}$ ), albeit with a reduced cooperativity of $\mathrm{Na}^{+}$binding (Hill coefficient 1.3 vs. 2.1 for the wild-type). For A2-R593Q, A2-R908Q, and A3-D887Y, the apparent $\mathrm{Na}^{+}$affinity was close to wild-type. A3P972del likewise exhibited only slightly reduced apparent $\mathrm{Na}^{+}$affinity, but the cooperativity of $\mathrm{Na}^{+}$binding at the three sites was severely reduced (Hill coefficient 1.2 vs. 2.4 for the wild-type).

Dephosphorylation of NKA, liberating inorganic phosphate, occurs when two $\mathrm{K}^{+}$ions bind to the phosphorylated intermediate in the E2P form (reactions 5 and 6 in Fig. 5D). The $\mathrm{K}^{+}$binding properties of the stably expressed mutants showing NKA pump activity (A2-I293M, A2-R593Q, A2-R908Q, A3-P972del, and A3-D887Y) were addressed by determining the $\mathrm{K}^{+}$dependence of NKA pump activity (figure S10), which for all these mutants indicated wild-type-like or slightly higher apparent $\mathrm{K}^{+}$affinity for activation. The $\mathrm{K}^{+}$concentration dependence of the wild-type shows in addition to the activation phase reflecting $\mathrm{K}^{+}$binding to the E2P phosphoenzyme intermediate also an inhibition phase at high $\mathrm{K}^{+}$concentrations, reflecting competition of $\mathrm{K}^{+}$with $\mathrm{Na}^{+}$for the $\mathrm{E} 1$ sites (Toustrup-Jensen et al., 2014) This inhibition was more pronounced for A2-I293M, A2-R593Q, and A3$\mathrm{D} 887 \mathrm{Y}$, relative to wild-type, indicating a reduced $\mathrm{Na}^{+} / \mathrm{K}^{+}$selectivity ratio, whereas the opposite was true for A3P972del, as also indicated by the increased dephosphorylation rate observed for this mutant in the presence of $\mathrm{Na}^{+}$ without $\mathrm{K}^{+}$(Supplementery Fig. 12). We assessed the $\mathrm{K}^{+}$affinity of the transiently expressed mutants showing robust phosphorylation (A2-G366A, A3-L292R, and A3-G316V) by determining the ability of $\mathrm{K}^{+}$to prevent accumulation of phosphoenzyme (Fig. 6B). These three mutants exhibited a dramatic $>15$-fold reduction of apparent $\mathrm{K}^{+}$affinity. In fact, the E2P phosphoenzyme intermediate of A2-G366A was totally insensitive to $\mathrm{K}^{+}$.

\section{Functional characterization of $A T P 1 A 2-A 3$ heterozygous mutations - E1-E2 and E1P-E2P conformational changes}

The mutational effects on the E1-E2 and E1P-E2P distributions of the respective dephospho- and phospho-forms of selected mutants were also addressed (Supplementary Fig. 12). To assess a shift of the distribution between the dephosphoenzyme conformations E1 and E2 we determined the ATP concentration dependence of NKA pump activity (Supplementary Fig. 12A). Because E1 binds ATP with higher affinity than E2 (Fig. 5D), a shift of the E1-E2 distribution results in a change of the apparent affinity for ATP. Mutant A2-I293M exhibited a pronounced increase of apparent ATP affinity relative to the wild-type, indicating a shift of the E1-E2 distribution in favour of E1. Mutants A2-R593Q and A3-D887Y were also E1-shifted, but to a lesser extent, whereas A2-R908Q and A3P972del were E2-shifted (reduced apparent ATP affinity). To assesss the distribution of the phosphoenzyme between E1P and E2P (reaction 4 in Fig. 5D), we took advantage of the ADP-sensitivity of E1P (Supplementary 
Fig. S12B). Upon addition of ADP to the phosphoenzyme formed in the presence of $\mathrm{Na}^{+}$and absence of $\mathrm{K}^{+}$, two clearly distinguishable phases of the dephosphorylation reaction are observed for the wild-type. The rapid phase reflects E1P, donating the phosphoryl group back to ADP (reversal of reaction 3 in Fig.5D), forming ATP, whereas the slow phase reflects hydrolysis of E2P (reaction 6 in Fig. 5D), which proceeds slowly in the absence of activation by $\mathrm{K}^{+}$. However, the A2-G366A mutant exhibited only one phase, corresponding to E2P, indicating that all phosphoenzyme accumulated as E2P, which in conjunction with the $\mathrm{K}^{+}$insensitivity of the phosphoenzyme (Fig. 6B) indicates inability to dephosphorylate and, hence, to catalyze ATP hydrolysis, in accordance with its strategic position in the P-domain between the P1-helix and the P1- $\beta$-strand leading directly to the phosphorylated aspartate. Mutant A2-I293M exhibited two phases like the wild-type, but a pronounced increase of the amplitude of the rapid phase relative to the wild-type was seen, indicating a conformational shift toward E1P. Mutants A2R593Q and A3-P972del showed a less pronounced E1P shift. Hence, both A2-I293M and A2-R593Q exhibited parallel shifts of the dephosphoenzyme and phosphoenzyme conformational equilibria in favour of E1/E1P. The strong effect of A2-I293M on the conformational equilibria is consistent with the predicted clash of the methionine with hydrophobic residues in the E2 form (Supplementary Fig. S4). It is furthermore of note that A3-P972del exhibited a 3-fold faster rate of the slow phase, representing E2P, relative to wild-type, indicating that in the absence of $\mathrm{K}^{+}, \mathrm{Na}^{+}$is able to activate dephosphorylation to some extent, i.e. the $\mathrm{Na}^{+} / \mathrm{K}^{+}$selectivity ratio was increased.

\section{Supplementary legend to figure 5}

The reaction cycle of NKA is shown as six numbered partial reactions. Three intracellular $\mathrm{Na}^{+}$are exchanged for two extracellular $\mathrm{K}^{+}$in each cycle resulting in hydrolysis of one ATP molecule. E1 and E1P are $\mathrm{Na}^{+}$selective conformations, whereas E2 and E2P are $\mathrm{K}^{+}$selective conformations. Phosphorylation by ATP (indicated by “P”) of a conserved aspartate residue in the P-domain (reaction 3) is triggered when E1 binds three cytoplasmic $\mathrm{Na}^{+}$ (reaction 2), whereas dephosphorylation (reaction 6) resulting in liberation of inorganic phosphate $\left(\mathrm{P}_{\mathrm{i}}\right)$ depends on the binding of two extracellular $\mathrm{K}^{+}$to E2P (reaction 5). During the transport process the ions become occluded (indicated by parentheses). The free ions are labeled c and e for cytoplasmic and extracellular, respectively. The ATP indicated in red is bound without being hydrolysed, accelerating the deocclusion of $\mathrm{K}^{+}$(reaction 1 ). 


\section{Supplementary Figures}
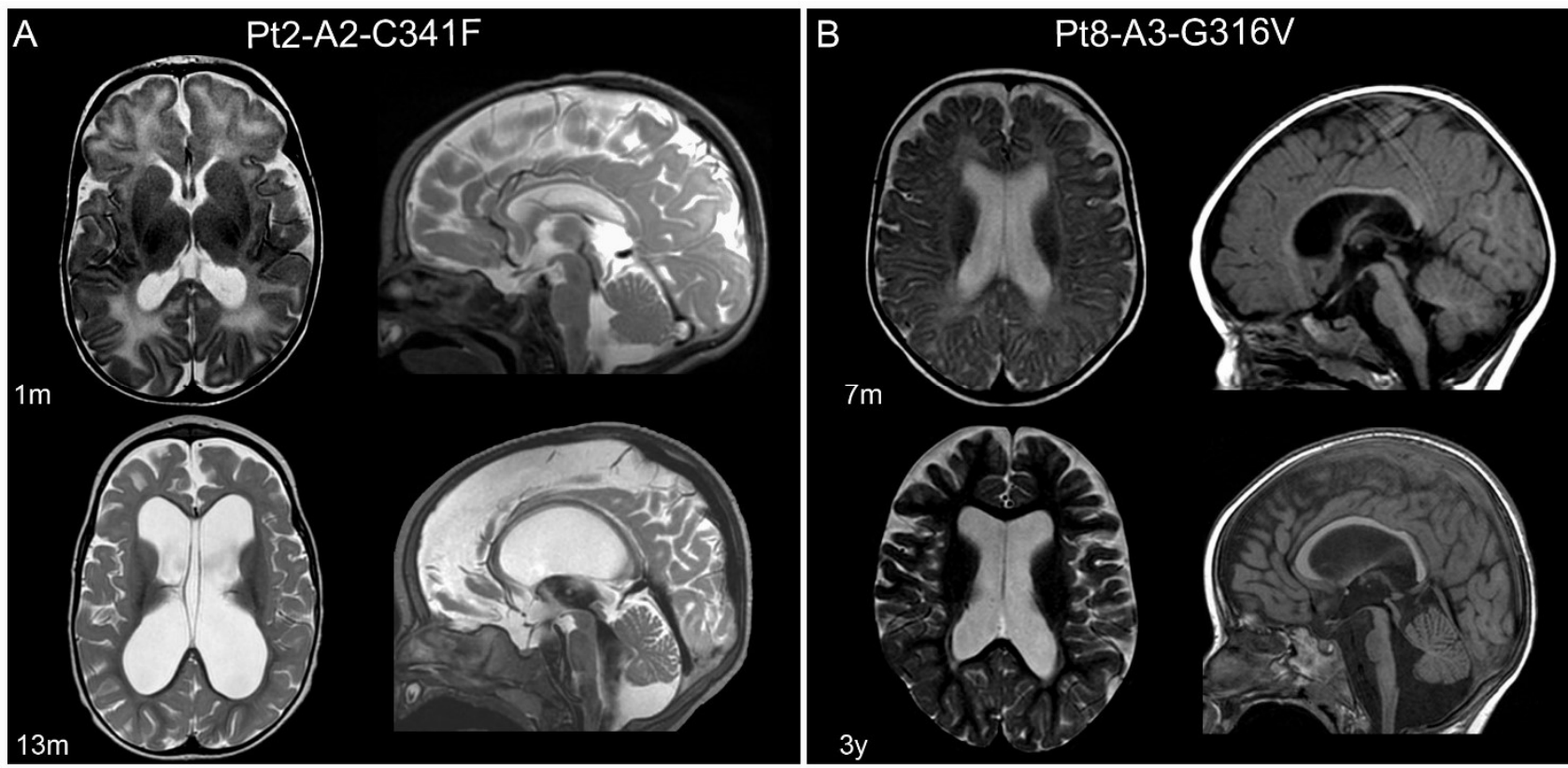

Supplementary Figure 1 - Brain MRI in two patients with progressive brain, brainstem and cerebellar atrophy.

Patient 2 (A). From left to right: axial and sagittal T2- weighted (W) images at age one month (top); axial and sagittal T2-W images at age 13 months (bottom). Patient 8 (B). From left to right: axial T2-W and sagittal T1-W images at age seven months (top); axial T2-W and sagittal T1-W at age three years (bottom). In both patients, atrophic changes involving all brain structures can be appreciated in the second MRI scan. 

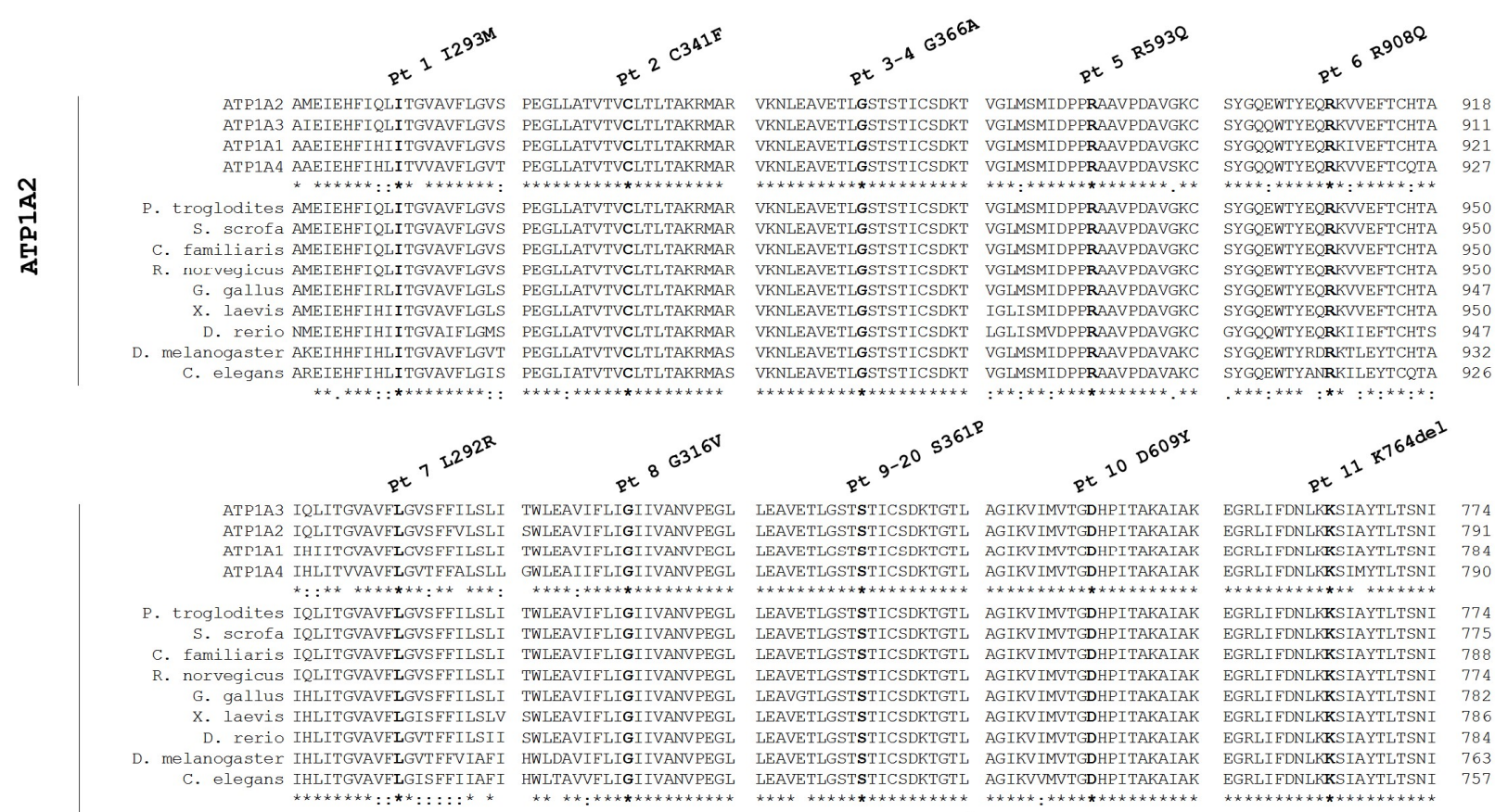

EGRLIFDNLKKSIAYYTLTSNI 774 EGRLIFDNLKKSIAYTLTSNI 791 EGRLIFDNLIKS IAYTLTSNI 784 * EGRLIFDNLKKSIAYTLTSNI 774 EGRLIFDNLKKSTAYTLTSNI 775 EGRLIFDNLKKSTAYTLTSNI 788 EGRLIFDNLKKSIAYTLTSNI 774 EGRLIFDNLKKSIAYTLTSNI 782 EGRLIFDNLKKSIAYTLTSNI $\quad 786$ EGRLIFDNLKKSIAYTLTSNI 784 EGRLIFDNLKKSTAYTLTSNI 76 EGRLIIDNLKKSIAYTLTSNI 75<smiles>[Te]=[W]=[W]</smiles>

$P^{+} 1308015$ $\begin{array}{ll}\text { ATP1A3 SIAYTLTSNIPEITPFLIFIM } & \text { PLGTITILCIDLGTDMVPAIS } \\ \text { ATP1A2 SIAYTLTSNIPEITPFLIFII } & \text { PLGTVTILCIDLGTDMVPAIS }\end{array}$ ATP1A2 SIAYTLTSNIPEITPFLLFII PLGTVTILCIDLGTDMVPAIS
ATP1A1 SIAYTLTSNIPEITPFLIFII PLGTVTILCIDLGTDMVPAIS ATP1A1 SIAYTLTSNIPEITPFLIFII PLGTVTILCIDLGTDMVPAI
ATP1A4 SIMYTLTSNIPEITPFLMFII PLGTITILIDLGTDMVAIS

P. troglodites SIAYTLTSNIPEITPFLLFIM PLGTITILCIDLGTDMVPAIS S. scrofa SIAYTLTSNIPEITPFLLFIM PLGTITILCIDLGTDMVPAIS
C. familiaris SIAYTLTSNIPEITPFLIFIM PLGTITILCIDLGTDMVPAIS R. norvegicus SIAYTLTSNIPEITPFLLFIM PLGTITILCIDLGTDMVPAIS G. gaIlus SIAYTLTSNIPEITPFLIFII PLGTCTILCIDLGTDMVPAI . laevis SIAYTLTSNIPEITPFLLFIM PLGTITILCIDLGTDMVPAI D. melanogaster SIAYTLTSNIPEIS PFLAAFIL PLGTVTILCIDLGTDMI PAIS

c. elegans SIAYTLTSNIPEISPFLTYIL PLGTVTILCIDLGTDMVPAIS
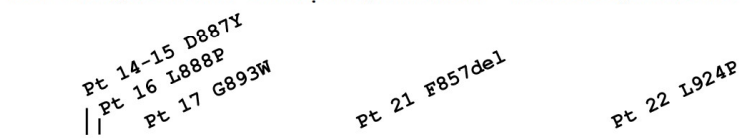

WDDRTVNDLEDSYGQQWTYEQ IGMIQALGGFFSYFVILAENG $\begin{array}{ll}\text { WDDRTMNDLEDSYGQEWTYEQ } & \text { IGMIQALGGEFTYFVILAENG } \\ \text { WDDRWINDVEDSYGQQWTYEQ } & \text { IGMIOALGGFFTYFVILAENG }\end{array}$ WEDKYLNDLEDSYGQQWTYEQ IGMIQALAGFFTYFVILAENG $:::^{* *}: * \star * * * *: * * * * * * *: \quad * * * * * * * . * * *: * * * * * * * * *$ WDDRTVNDLEDSYGQQWTYEQ IGMIQALGGEFSYFVILAENG WDDRTVNDLEDSYGQQWTYEQ IGMIOALGGFFSYFVILAEN $\begin{array}{ll}\text { WDDRTVDLEDSYGQQWTYEQ } & \text { IGMLQALGGFFSYFVILAENG } \\ \text { WDDRVNDLEDSYQQWTYEQ } & \text { IGMIAAGGFFSYFVILAENG }\end{array}$ WDDRW INDVEDSYGQWTFEQ IGMIQALGGEFTYFVIMAENG WDDRSVNDLEDSYGQQWTYEQ IGMIQALGGFFAYFVILAENG WDDRSNNDLEDSYGQQWTYEQ IGMIQALGGFFSYFVILAENG WDSKAVNDLTDSYGQEWTYRD IGMIQAAAGFFVYFVIMAENG WDSRAYNNVLDSYGQEWTYAN IGMIQASAGFFTYFWIMADNG

ASIVVVQWADLIICKTRRNSV 93 VSIVVVQWADLIICKTRRNSV 941 VSIVUVWDLVICKTRRNSV 94 VITVVQWADLIISKTRRNSL 950 VSTVVVOWADITICKTRRNSV

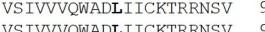
VSIVVVQWADLIICKTRRNSV 93 VS IVVVVWADL I TCKTRRNSV 94 VSTVVVVOWADT ITCKTRRNSV 934 VSIVVVQWADVIICKTRRNSV 94 VSIVVVOWADVIICKTRRNSV 94 ISIVVVQWADLIICKTRRNSI 923 VSIVVVQWADLIISKTRRNSL 9

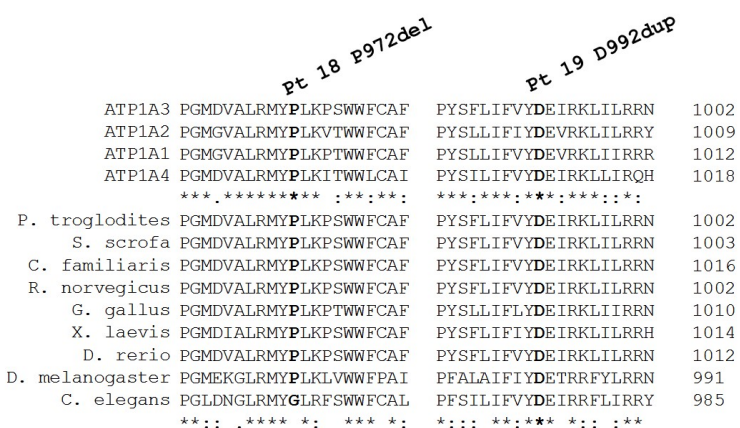

\section{Supplementary Figure 2 - Multiple sequence alignment and conservation analysis}

The protein sequence of the four paralogs of the $\alpha$ subunit (ATP1A1-4) and of the ATP1A2/ATP1A3 orthologs in nine different species (Pan troglodytes, Sus scrofa, Canis familiaris, Rattus norvegicus, Gallus gallus, Xenopus laevis, Danio renio, Drosophila melanogaster, Caenorabditis elegans) are shown, with the mutated residues in bold. The details of the ATP1A2 and ATP1A3 disease mutations in the cohort are displayed above the alignments. The asterisk indicates positions which have a single, fully conserved residue between all the input sequences, the colon indicates conservation between groups of strongly similar properties, and the period indicates conservation between groups of weakly similar properties. 

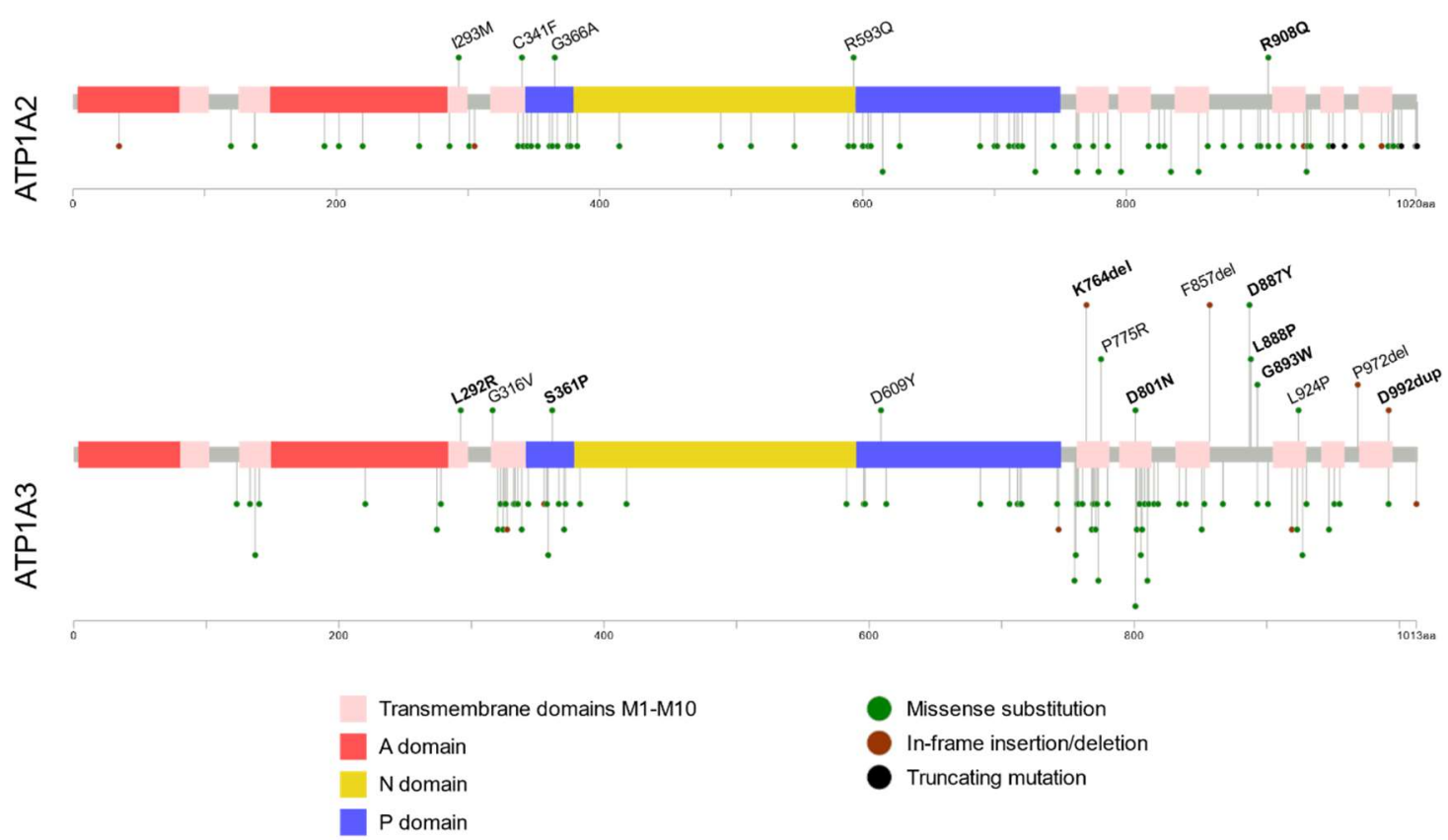

Supplementary Figure 3 Distribution of the ATP1A2 and ATP1A3 disease mutations on linear protein map.

The mutations observed in our cohort are shown and detailed at the top of each track, compared to previously published mutations, associated with classical ATP1A2-/ATP1A3-phenotypes, which are shown at the bottom. Mutations associated with polymicrogyria in our cohort are in bold. The different domains of the ATP1A2 and ATP1A3 proteins are represented as coloured box: cytoplasmic domains $\mathrm{P}$ (blue boxes), $\mathrm{N}$ (yellow box), and A (red boxes), and transmembrane helices M1-M10 (pink boxes). The mutations are represented as green (missense substitutions), brown (in-frame insertions/deletions) and black (truncating mutations) dots. 

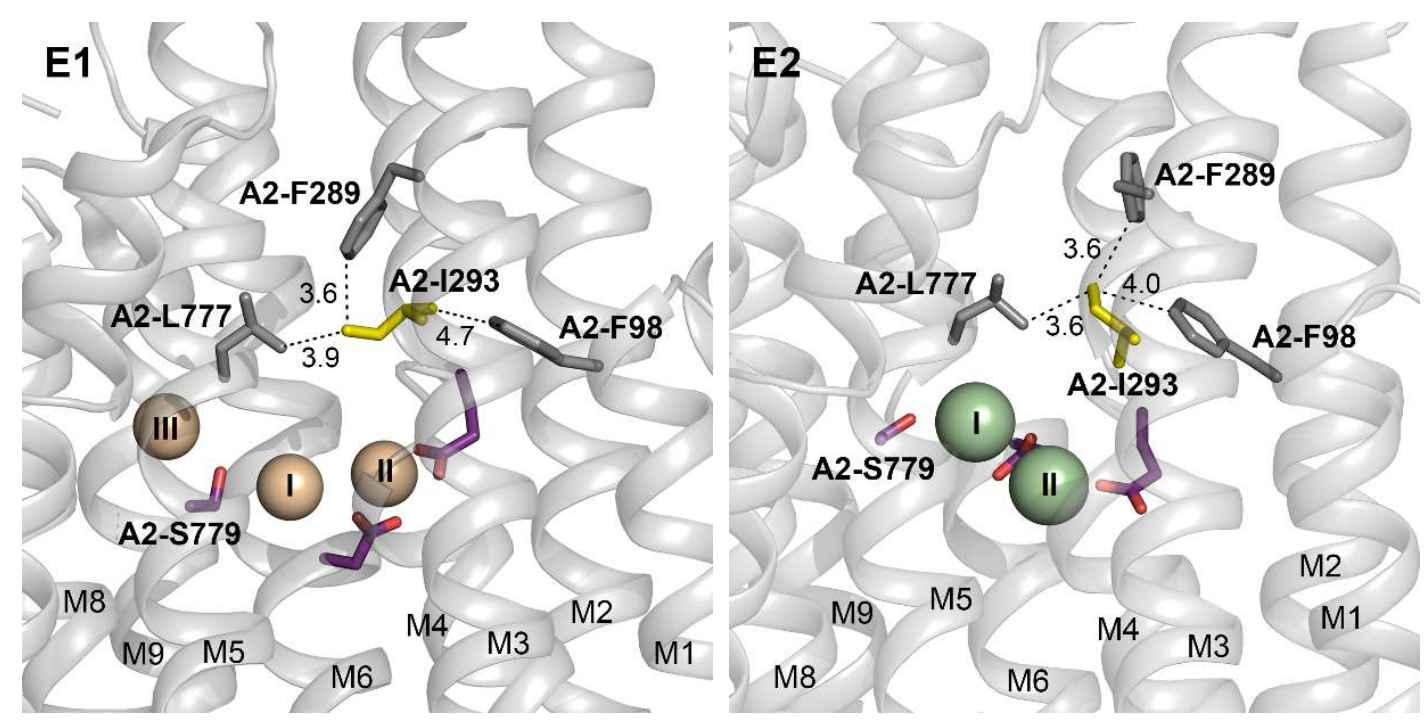

\section{Supplementary Figure 4 - Structural analysis of A2-I293}

The side chain of the leucine replaced by methionine in mutant A2-I293M is shown in yellow in the structures of the NKA pump $\alpha 1$-subunit in the $\mathrm{Na}^{+}$-bound $\mathrm{E} 1$ form (PDB ID $3 \mathrm{WGV}$, chain A) and the $\mathrm{K}^{+}$-bound E2 form (PDB ID 2ZXE) in side view of the transmembrane helices (labelled M1-M8) along the plane of the membrane. The cytoplasmic side is up. The three $\mathrm{Na}^{+}$ions in the E1 form are shown as golden spheres labelled I, II, and III, and the two $\mathrm{K}^{+}$ions in the E2 form are shown as green spheres labelled I and II (Shinoda et al., 2009; Kanai et al., 2013). Some of the residue side chains donating liganding groups for ion binding are shown in purple (oxygen red). A2-I293 is located in the M3 transmembrane helix. The substitution with methionine causes $1 \AA$ elongation of the side chain potentially disturbing $\mathrm{Na}^{+}$and $\mathrm{K}^{+}$binding. The clash with other hydrophobic side chains (carbon atoms shown in grey with distance to A2-I293 in $\AA$ ) including A2-L777 and A2-F98 at the inlet of the ion binding pocket will be most pronounced in E2, thus explaining the E1 shift. 


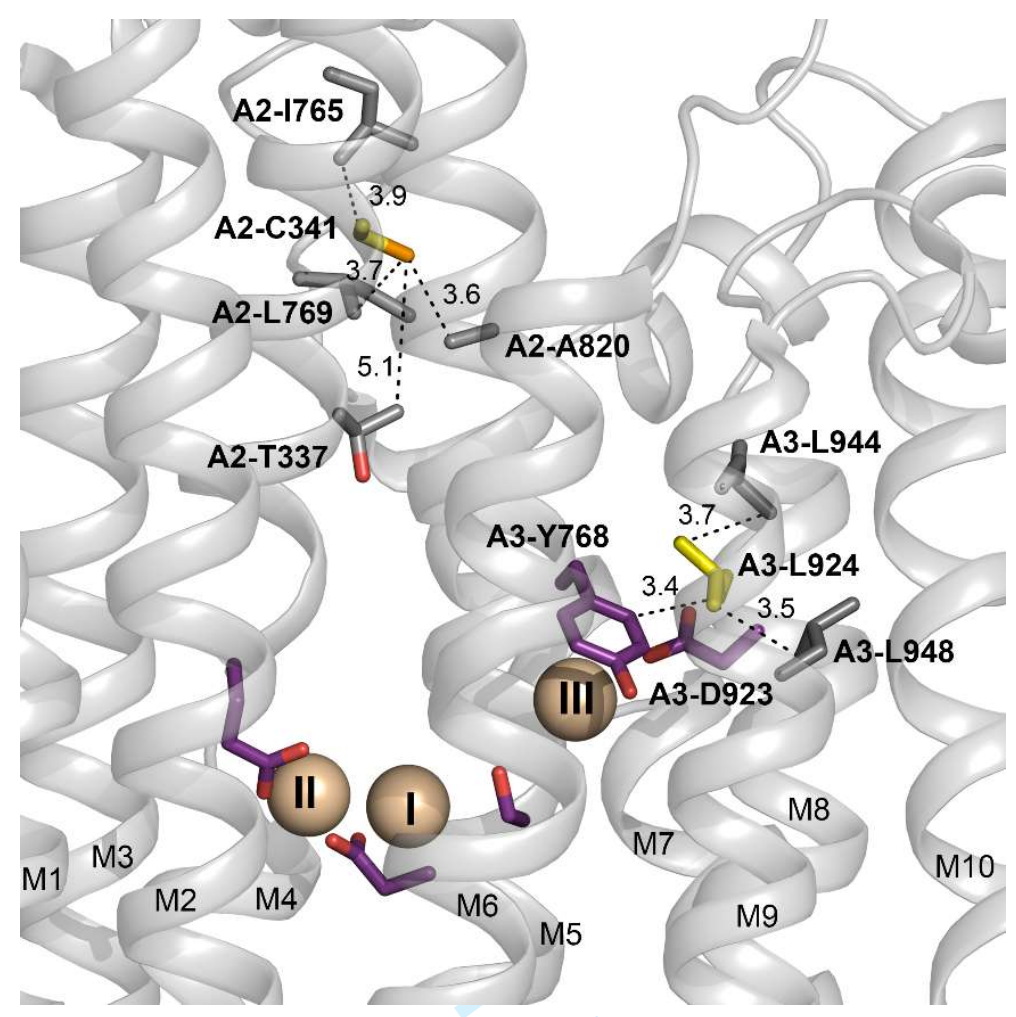

\section{Supplementary Figure 5 - Structural analysis of A2-C341 and A3-L924}

The side chains of the cysteine and leucine replaced by phenylalanine and proline in mutants A2-C341F and A3L924P are shown in yellow (sulfur orange) in the structure of the NKA pump $\alpha 1$-subunit in the $\mathrm{Na}^{+}$-bound E1 form (PDB ID 3WGV, chain A) in side view of the transmembrane helices (labelled M1-M6, M8, and M9) along the plane of the membrane. The cytoplasmic side is up. The three $\mathrm{Na}^{+}$ions are shown as golden spheres labelled I, II, and III. Some of the residue side chains donating liganding groups for ion binding are shown in purple (oxygen red). A2-C341 is located in the M4 transmembrane helix connecting the ion binding sites with the P1-helix of the P-domain leading to the phosphorylated aspartate. The bulky phenylalanine substituent in mutant A2-C341F will clash with residues in M5 and top of M6 (some shown here with distance to A2-C341 in $\AA$, carbon grey, oxygen red) likely disturbing helix packing with impact on protein folding and the ion binding sites formed by residues in M4, M5, and M6 and their functional interaction with the phosphorylation site. A3-L924 is located in M8 right besides A3-D923, which participates in $\mathrm{Na}^{+}$binding at site III (Einholm et al., 2010). Moreover A3-L924 interacts with A3-Y768, also part of site III. Replacement of A3-L924 with proline will break the M8 helix, and interfere with the "zipper" interaction with hydrophobic residues A3-L944 and A3-L948 of M9, thereby disturbing $\mathrm{Na}^{+}$ binding at site III (Arystarkhova et al., 2019). 


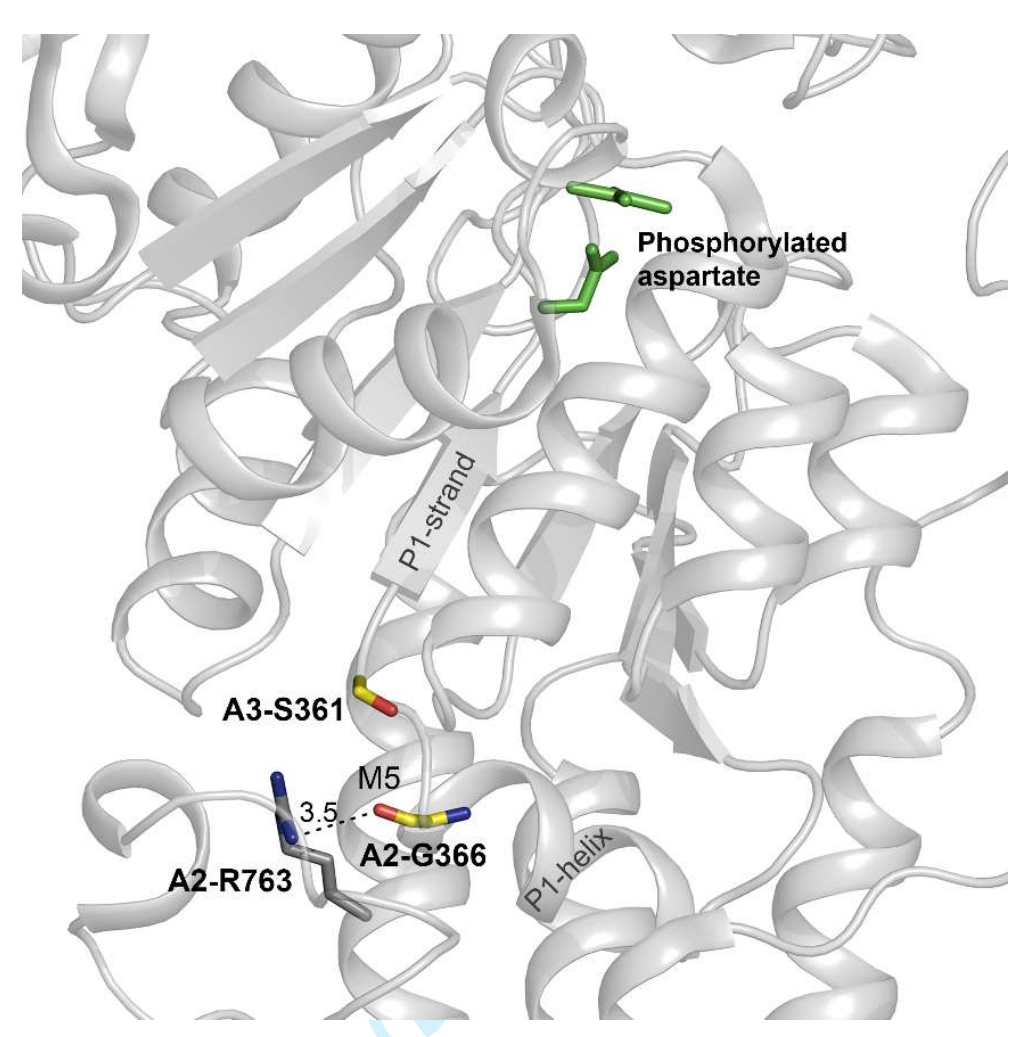

\section{Supplementary Figure 6 - Structural analysis of A2-G366 and A3-S361}

The side chains of the glycine and serine replaced by alanine and proline in mutants A2-G366A and A3-S361P, respectively, are shown in yellow (oxygen red, nitrogen blue) in the structure of the NKA pump $\alpha 1$-subunit in the $\mathrm{Na}^{+}$-bound E1 form (PDB ID 3WGV, chain A) in side view of the cytoplasmic P-domain along the plane of the membrane. A2-G366 is at the junction between the P1-helix and the P1- $\beta$-strand leading directly to the phosphorylated aspartate (D374 in A2) shown in green (phosphate analog $\mathrm{AlF}_{4}$ ). The glycine is important for the direction of this $\beta$-strand and forms a hydrogen bond with R763 at the top of M5 (side chain indicated with grey carbon atoms and blue nitrogen, distance to A2-G366 in $\AA$ ). The P1-helix extends from the M4 transmembrane helix, which like M5 is involved in ion binding. Hence, replacement of the glycine may affect both the function of the phosphorylation site and the ion binding sites. A3-S361 is located at the beginning of the P1- $\beta$-strand, five residues from the phosphorylated aspartate (D366 in A3). Proline substitution of the serine is expected to change the orientation of the P1- $\beta$-strand and thereby interfere with phosphorylation. 


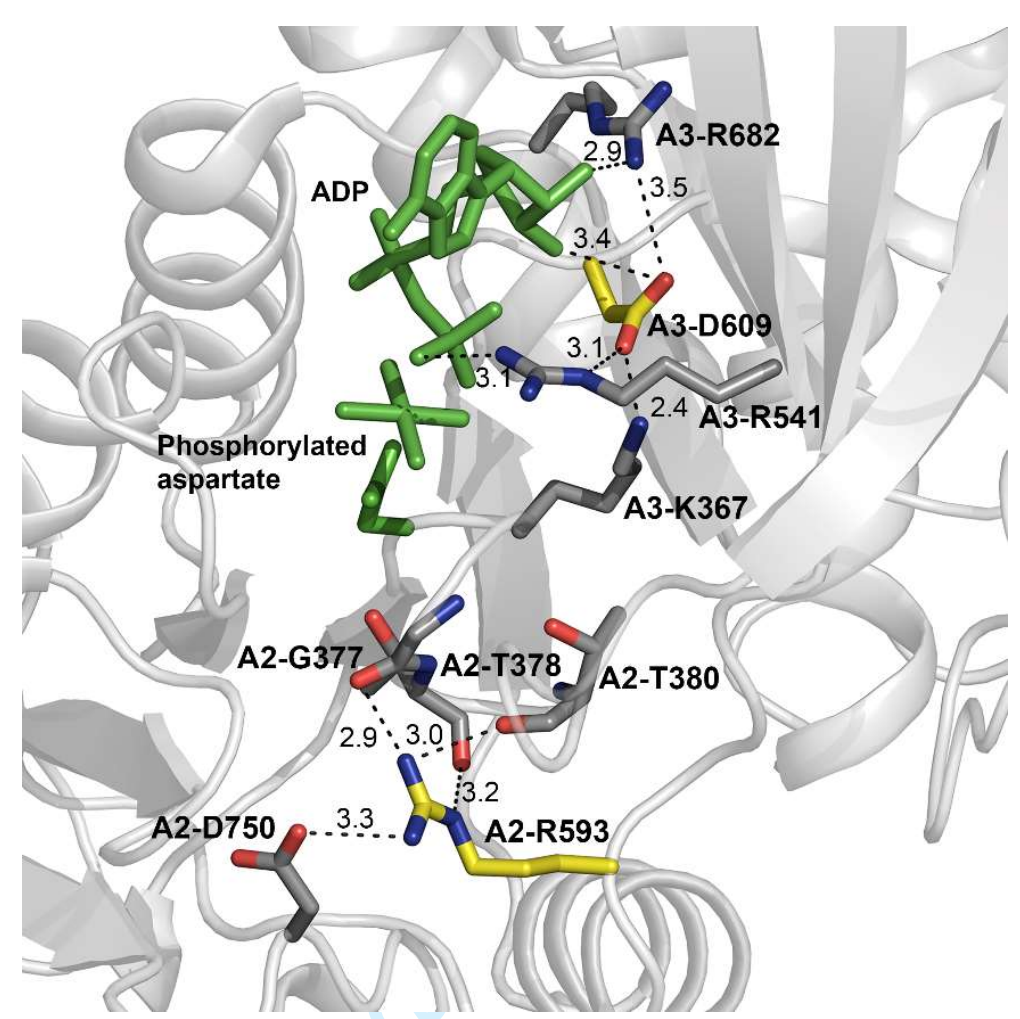

\section{Supplementary Figure 7 - Structural analysis of A2-R593 and A3-D609}

The side chains of the arginine and aspartate replaced by glutamine and tyrosine in mutants A2-R593Q and A3D609Y, respectively, are shown in yellow (oxygen red, nitrogen blue) in the structure of the NKA pump $\alpha 1$ subunit in the $\mathrm{Na}^{+}$-bound $\mathrm{E} 1$ form (PDB ID 3WGV, chain A) in top view of the P-domain from the cytoplasmic side. Both A2-R593 and A3-D609 are located near the bound nucleotide and phosphorylated aspartate (shown in green, phosphate analog $\mathrm{AlF}_{4}$ ). A2-R593 forms hydrogen bonds to backbone carbonyls of A2-G377, A2-T378, and A2-T380 close to the phosphorylated aspartate (D374 in A2), and a salt bridge to the side chain of A2-D750 (residues shown with carbon grey, oxygen red, nitrogen blue, and bond distances in $\AA$ ). These bonds are important for the configuration of the phosphorylation site. A3-D609 is part of the TGD motif at the ATP binding site. The aspartate forms bonds to the nucleotide as well as to conserved arginine residues and a lysine (A3-R541, A3-R682, and A3-K367) in the P- and N-domains involved in ATP binding (residues shown with carbon grey, oxygen red, nitrogen blue, and bond distances in $\AA$ ). Substitution with the bulky, aromatic tyrosine is expected to disrupt ATP binding/phosphorylation. 


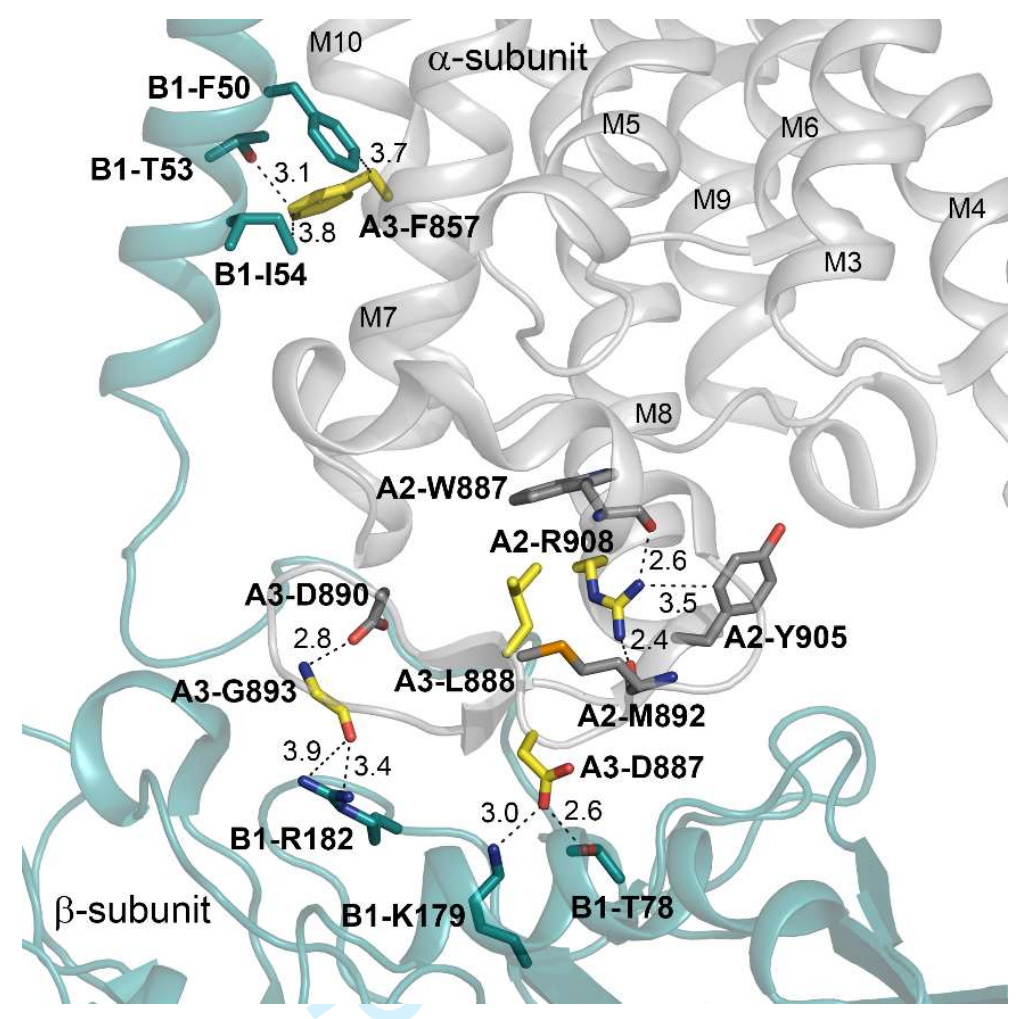

\section{Supplementary Figure 8 - Structural analysis of A3-F857, A3-D887, A3-L888, A3-G893, and A2-R908}

The side chains of the deleted phenylalanine (A3-F857del), and the aspartate, leucine, glycine, and arginine replaced by tyrosine, proline, tryptophan, and glutamine in mutants A3-D887Y, A3-L888P, A3-G893W, and A2R908Q, respectively, are shown in yellow (oxygen red, nitrogen blue) in the structure of the NKA $\alpha 1$-subunit in the $\mathrm{Na}^{+}$-bound $\mathrm{E} 1$ form (PDB ID $3 \mathrm{WGV}$, chain A) in side view of the extracellular domain along the plane of the membrane. These residues are located in M7 (A3-F857) and in the L7-8 loop connecting M7 and M8 on the extracellular side. A3-F857 interacts in a "sandwich" arrangement with B1-F50, B1-T53, and B1-I54 in the transmembrane helix of the $\beta$-subunit ( $\beta 1$-isoform, teal color, PDB ID $3 \mathrm{WGV}$, chain B). The deletion of A3-F857 likely disturbs the $\alpha \beta$ interaction, both directly, and indirectly by affecting the position of the L7-8 loop, which interacts with the extracellular domain of the $\beta$-subunit. The $\alpha \beta$ interaction involving the L7-8 loop will also be disturbed by the mutations in this loop. A3-D887 forms a salt-bridge to B1-K179 and a hydrogen bond with B1T78. The bulky tyrosine substituent will disrupt these bonds. Replacement of A3-L888 with proline will redirect the orientation of the L7-8 loop and thereby interfere with the interaction with the $\beta$-subunit. A3-G893 is part of the SYGQ motif known to interact with the $\beta$-subunit long before the atomic structure was available (Geering, 2001). The backbone carbonyl group of A3-G893 forms hydrogen bonds with B1-R182, and the backbone nitrogen is hydrogen bonded to A3-D890, thereby stabilizing the fold of the L7-8 loop. The bulky tryptophan substituent will interfere with this interaction. A2-R908 is hydrogen bonded to backbone carbonyls of A2-W887 and A2M892 and probably interacts with the aromatic ring of A2-Y905 in this loop as well, stabilizing the loop. 


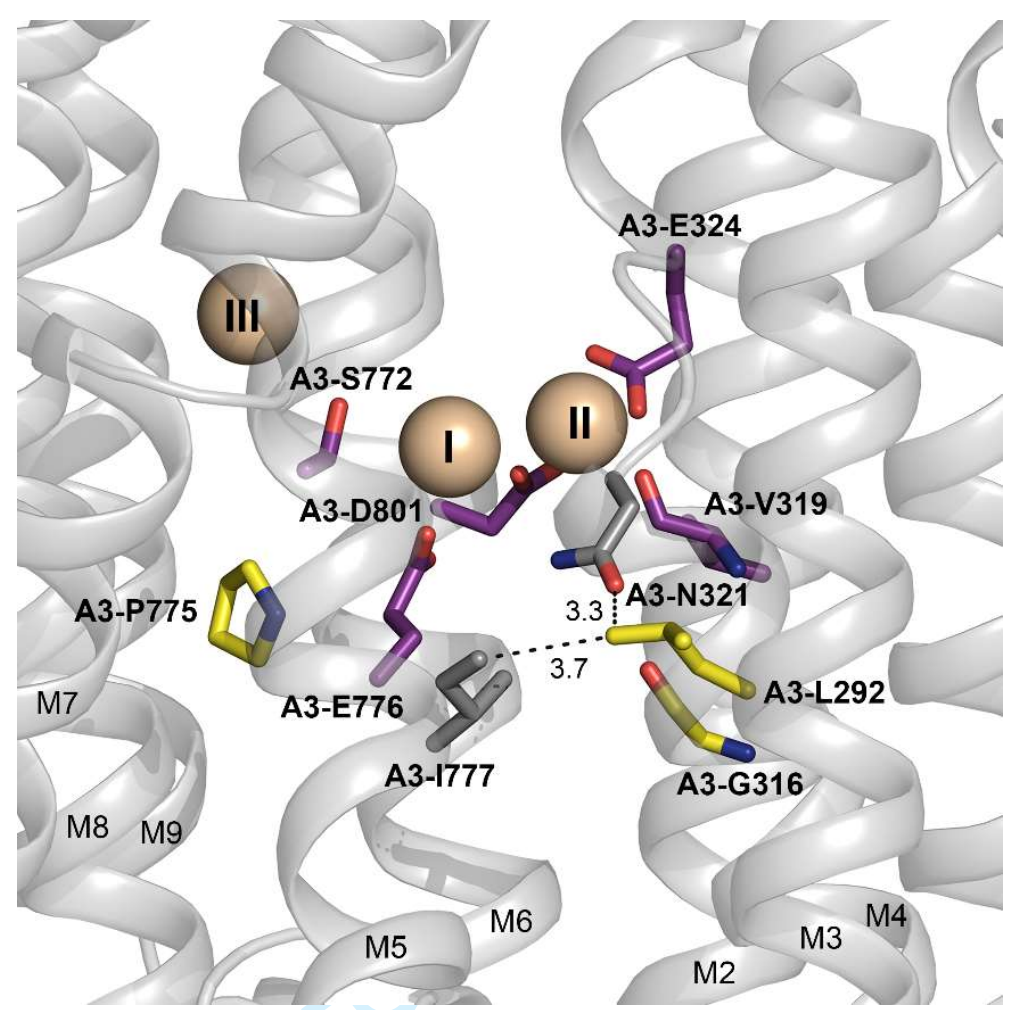

\section{Supplementary Figure 9 - Structural analysis of A3-L292, A3-G316, A3-P775, and A3-D801}

The leucine, glycine, and proline replaced by arginine, valine, and arginine in mutants A3-L292R, A3-G316V, and A3-P775R, respectively, are shown in yellow (oxygen red, nitrogen blue) in the structure of the NKA pump a1-subunit in the $\mathrm{Na}^{+}$-bound $\mathrm{E} 1$ form (PDB ID $3 \mathrm{WGV}$, chain A) in side view of the transmembrane helices (labelled M2-M9) along the plane of the membrane. Cytoplasmic side up. The aspartate replaced by asparagine in mutant A3-D801N is located in transmembrane helix M6, and is directly involved in binding of $\mathrm{Na}^{+}$and $\mathrm{K}^{+}$at sites I and II. It is therefore shown in purple (oxygen red) together with A3-E324, A3-V319, A3-S772, A3-E776, and A3-D801, which likewise donate liganding groups for $\mathrm{Na}^{+}$and $\mathrm{K}^{+}$binding (three $\mathrm{Na}^{+}$ions are shown as golden spheres labelled I, II, and III). A3-L292, located in the M3 transmembrane helix, points toward M4 and M5 (distances shown in $\AA$ ), and its replacement with the bulky and positively charged arginine disturbs residues of M4 and M5 forming the ion binding sites. A3-G316V is located before the unwounded part of transmembrane helix M4, where the ions bind at A3-E324 (site II). A bulky valine replacing A3-G316 will clash with residues in M5 and M6 and redirect the M4 helix, thereby affecting ion binding. A3-P775 is located in M5 right beside the glutamate A3-E776 involved in ion binding. Replacement with arginine will interfere with the effect of proline (helix breaker) on the helix orientation, and the positive and bulky side chain of the arginine will clash with surrounding residues, thus further disturbing helix packing as well as $\mathrm{Na}^{+}$and $\mathrm{K}^{+}$binding. 


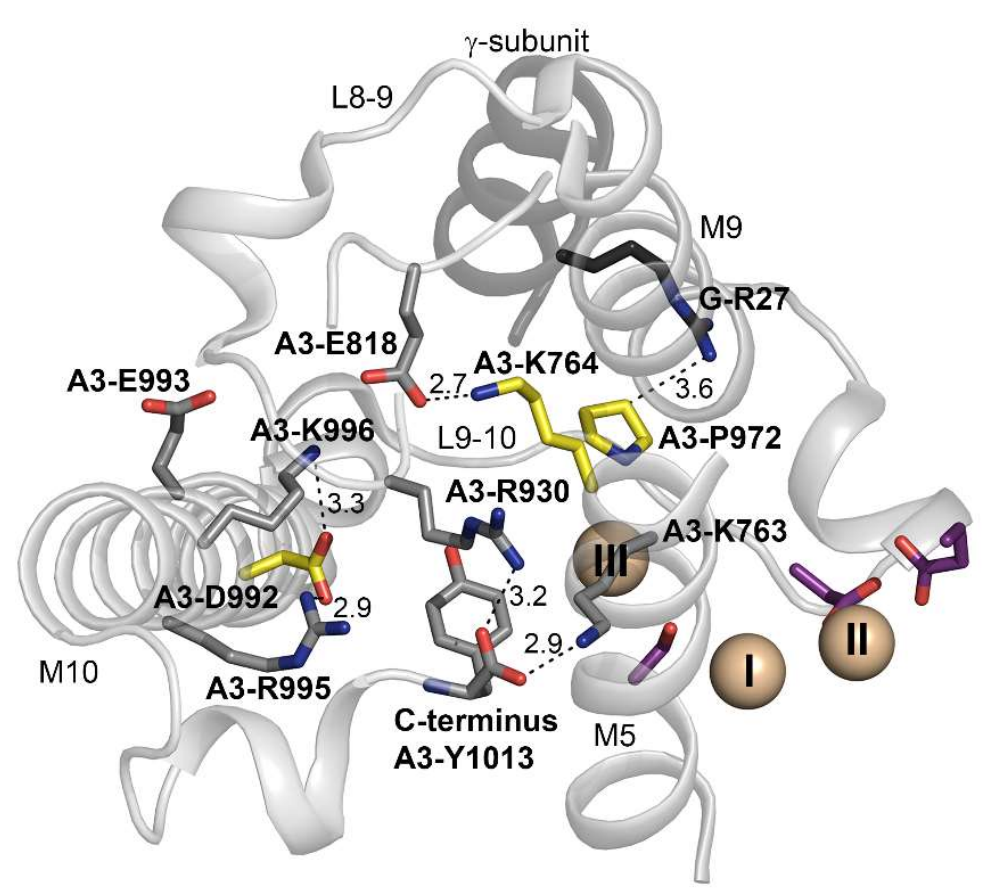

\section{Supplementary Figure 10 - Structural analysis of A3-K764, A3-P972, and A3-D992}

The lysine, proline, and aspartate deleted or duplicated in mutants A3-K764del, A3-P972del, and A3-D992dup are shown in yellow (oxygen red, nitrogen blue) in the structure of the NKA pump $\alpha 1$-subunit in the $\mathrm{Na}^{+}$-bound E1 form (PDB ID $3 \mathrm{WGV}$, chain A) in top view of the transmembrane domain from the cytoplasmic side. The three $\mathrm{Na}^{+}$ions are shown as golden spheres labelled I, II, and III, and some of the residue side chains donating liganding groups for ion binding are shown in purple (oxygen red). A3-K764 is located at the cytoplasmic end of transmembrane helix M5 before the ion binding sites. It participates in a hydrogen-bonding network of importance for the positioning of M5 and the C-terminus, and, thus, the functioning of the ion binding sites (Toustrup-Jensen et al., 2009). The network includes a bond between A3-K764 and the residue A3-E818 found mutated in patients with CAPOS syndrome (Roenn et al., 2019). A3-P972 is located in the L9-10 loop connecting the transmembrane helices M9 and M10 on the extracellular side. Deletion will interfere with the turn of the loop and shorten the loop. Hence, the directions of M9 and M10 may be changed, affecting the connection between L8-9 and the C-terminus through A3-R930 (for role of M10, see A3-D992dup below). The configuration of the C-terminus is well-known to affect $\mathrm{Na}^{+}$binding at site III through A3-K763 in M5 (Toustrup-Jensen et al., 2009). In addition, the deletion of A3-P972 will affect its interaction with the $\gamma$-subunit (shown in dark grey, PDB ID 3WGV, chain G) through G$\mathrm{R} 27$, which may further disturb the function of the $\mathrm{Na}^{+}$sites controlled by the $\gamma$-subunit. A3-D992 is located in the cytoplasmic end of M10, where it participates in interactions that indirectly are critical to $\mathrm{Na}^{+}$binding [see above for A3-P972del and (Einholm et al., 2016)]. Hence, A3-D992 positions A3-K996, which through interaction with a backbone carbonyl of L8-9 affects A3-R930 and its connection to M5 and the ion binding sites via the Cterminus. Duplication of A3-D992 is expected to disturb these interactions and thereby $\mathrm{Na}^{+}$binding. Changes in the helix orientations will also disturb helix packing and folding, thereby explaining the low expression level. A3- 
D992 is target of the AHC mutation D992Y (Heinzen et al., 2012; Sasaki et al., 2014), and the equivalent ATP1A2 residue is targeted by the HM mutation A2-D999H (Fernandez et al., 2008). 


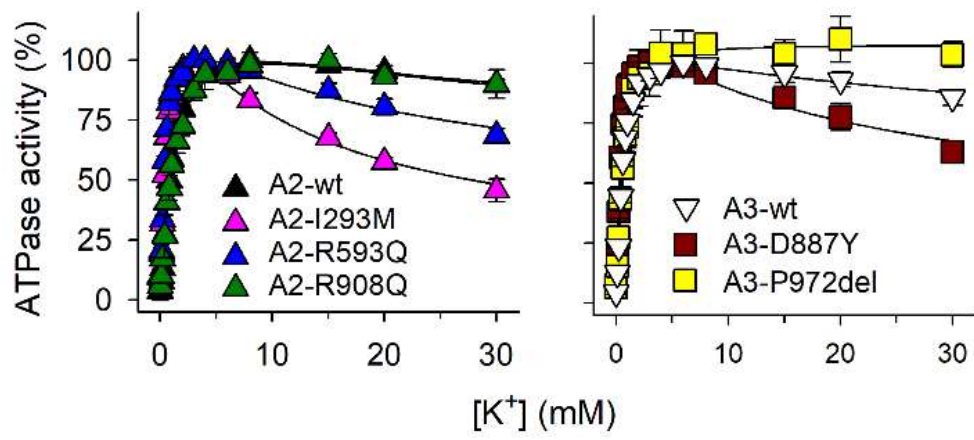

Supplementary Figure $11-\mathrm{K}+$ affinity determined from $\mathrm{K}+$ dependence of NKA activity on stably expressed NKA

ATPase activity was measured on stably expressed NKA enzyme at $37^{\circ} \mathrm{C}$ in $30 \mathrm{mM}$ histidine (pH 7.4$), 40 \mathrm{mM}$ $\mathrm{Na}^{+}, 3 \mathrm{mM} \mathrm{Mg}{ }^{2+}, 1 \mathrm{mM}$ EGTA, $3 \mathrm{mM}$ ATP, $10 \mu \mathrm{M}$ ouabain, and the indicated concentrations of $\mathrm{K}^{+}$. Symbols with error bars represent mean $\pm \mathrm{SD}$. The rising phase reflects the activation of dephosphorylation of E2P by binding of $\mathrm{K}^{+}$. The apparent $\mathrm{K}^{+}$affinities $\left(\mathrm{K}_{0.5}\right.$ values) corresponding to the rising phase were as follows: A2-wt, $759 \pm 22 \mu \mathrm{M}$; A2-I293M, $310 \pm 21 \mu \mathrm{M}$; A2-R593Q, $282 \pm 16 \mu \mathrm{M}$; A2-R908Q, $793 \pm 100 \mu \mathrm{M}$; A3-wt, $447 \pm 36$ $\mu \mathrm{M}$; A3-D887Y, $260 \pm 32 \mu \mathrm{M}$; A3-P972del, $592 \pm 35 \mu \mathrm{M}$. Hence, these mutants displayed an apparent $\mathrm{K}^{+}$ affinity similar to or slightly higher than that of the wild-type. The inhibition phase at high $\mathrm{K}^{+}$concentrations, reflecting competition of $\mathrm{K}^{+}$with $\mathrm{Na}^{+}$for the $\mathrm{E} 1$ sites was more pronounced for A2-I293M, A2-R593Q, and A3D887Y, relative to wild-type, indicating a reduced $\mathrm{Na}^{+} / \mathrm{K}^{+}$selectivity ratio. For A3-P972del an inhibition phase was not distinguishable, indicating an increased $\mathrm{Na}^{+} / \mathrm{K}^{+}$selectivity ratio, relative to wild-type (wt). 

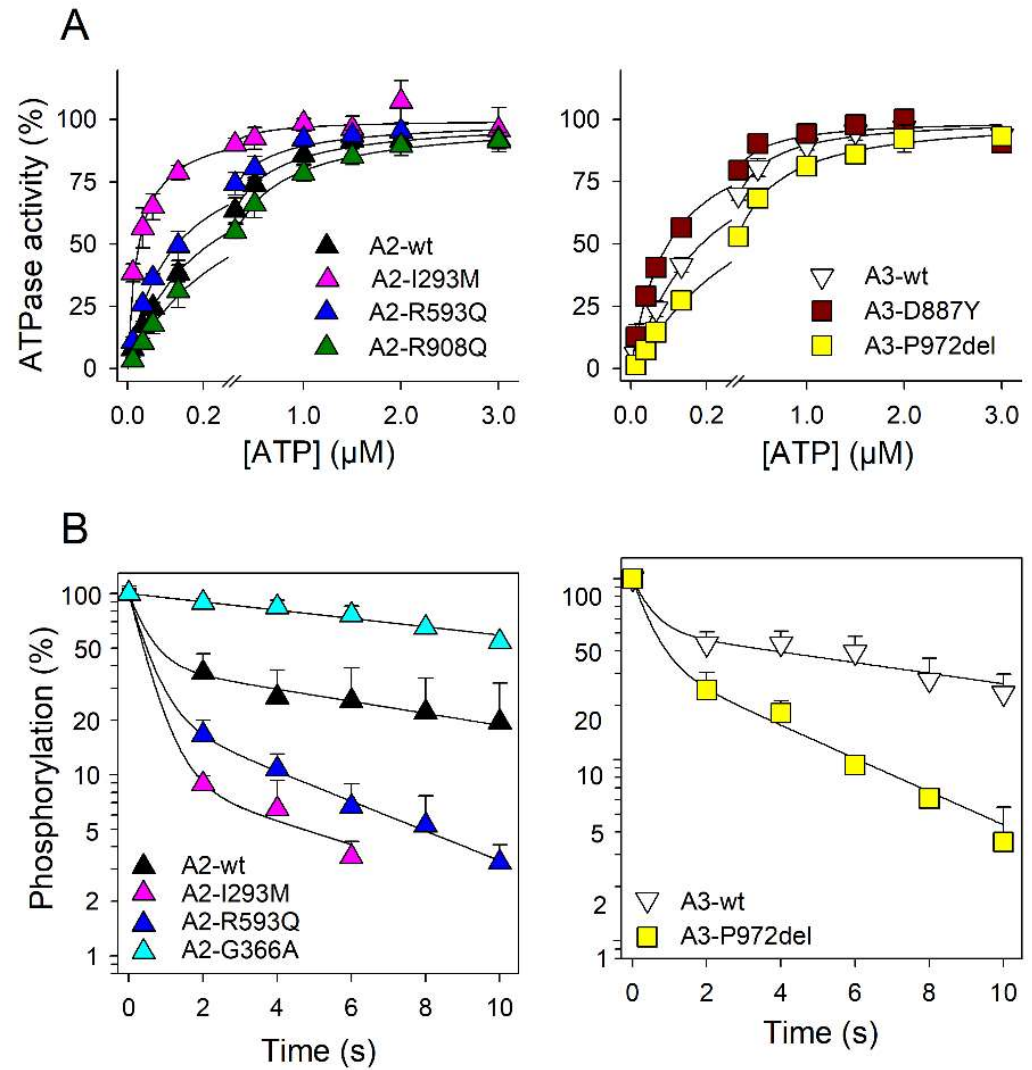

\section{Supplementary Figure 12 - Shift of E1-E2 and E1P-E2P conformational equilibria}

(A) Shift of the distribution between the dephosphoenzyme conformations E1 and E2 determined by apparent ATP affinity during steady-state enzyme turnover. Because E1 binds ATP with higher affinity than E2, shift of the E1E2 distribution results in a change of the apparent affinity for ATP, which was examined by studying the ATP dependence of NKA activity. Symbols with error bars represent mean \pm SD. Each line represents the best fit of a Hill function.(Nielsen et al., 2019) The apparent ATP affinities ( $\mathrm{K}_{0.5}$ values) are as follows: A2-wt, $158 \pm 20 \mu \mathrm{M}$; A2-I293M, $22 \pm 2 \mu \mathrm{M}$; A2-R593Q, $96 \pm 15 \mu \mathrm{M}$; A2-R908Q, $248 \pm 45 \mu \mathrm{M}$; A3-wt, $139 \pm 16 \mu \mathrm{M}$; A3-D887Y, 70 $\pm 9 \mu \mathrm{M}$; A3-P972del, $264 \pm 23 \mu \mathrm{M}$. (B) Shift of the distribution between the phosphoenzyme conformations E1P and E2P determined by ADP-sensitivity of the phosphoenzyme. Following phosphorylation of stably expressed (A2-I293M, A2-R593Q, A3-P972del) or transiently expressed (A2-G366A) NKA enzyme, ADP was added (at time " 0 ") to induce dephosphorylation, and the reaction was terminated at the indicated times. Symbols with error bars represent mean \pm SD. Each line shows the best fit of a bi-exponential decay function with the rapid phase representing the reaction of $\mathrm{ADP}$ with the ADP-sensitive E1P form and the slow phase representing dephosphorylation of the ADP-insensitive E2P form by hydrolysis. The A2-G366A mutant exhibited only one phase corresponding to E2P, therefore the best fit of a mono-exponential decay function is shown for this mutant. The extracted values for the E1P fractions of the phosphoenzyme (amplitude of the rapid phase) were: A2-wt, 58\%; A2-I293M, 91\%; A2-R593Q, 78\%; A2-G366A, 0\%; A3-wt, 48\%; A3-P972del, 62\%. Note also that A3- 
P972del exhibits a 3-fold faster rate of the slow phase, representing E2P, relative to wild-type, indicating that $\mathrm{Na}^{+}$ is able to activate dephosphorylation to some extent in the absence of $\mathrm{K}^{+}$, i.e. increased $\mathrm{Na}^{+} / \mathrm{K}^{+}$selectivity ratio. 


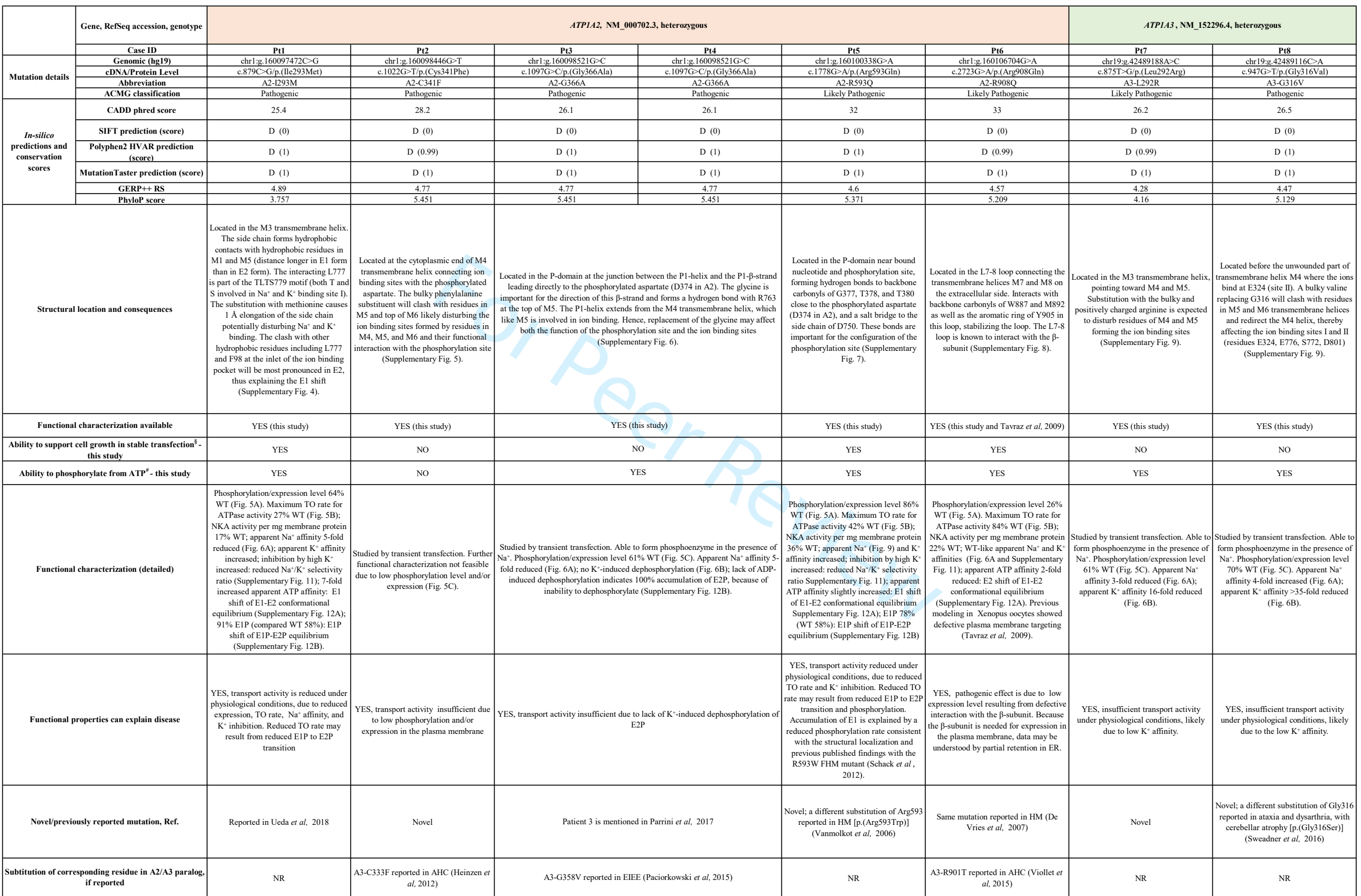




\begin{tabular}{|c|c|c|c|c|c|c|c|c|}
\hline \multicolumn{9}{|c|}{ ATPIA3, NM_152296.4, heteroroygous } \\
\hline 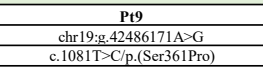 & 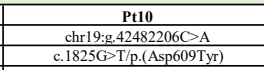 & 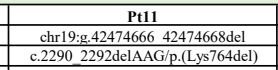 & 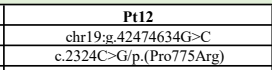 & 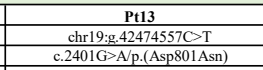 & 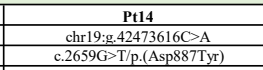 & 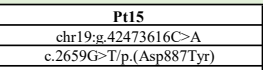 & 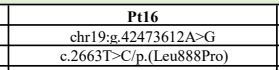 & 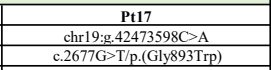 \\
\hline 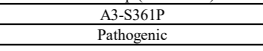 & 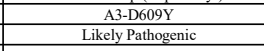 & 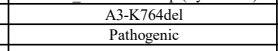 & 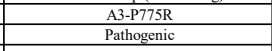 & $\begin{array}{l}\text { A3--3801N } \\
\text { Pathogenic } \\
\end{array}$ & 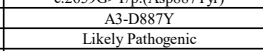 & 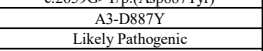 & 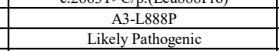 & 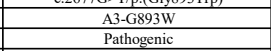 \\
\hline 25.8 & 32 & 21.3 & 25.4 & 29.9 & 25.7 & 25.7 & 26.5 & 28.1 \\
\hline $\mathrm{D}(0)$ & $\mathrm{D}(0)$ & $\mathrm{NA}$ & $\mathrm{D}(0)$ & $\mathrm{D}(0.001)$ & $\mathrm{D}(0)$ & $\mathrm{D}(0)$ & $\mathrm{D}(0)$ & $\mathrm{D}(0)$ \\
\hline P $(0.86)$ & $\mathrm{D}(1)$ & $\mathrm{NA}$ & $\mathrm{P}(0.84)$ & $\mathrm{D}(0.98)$ & P (0.88) & P $(0.88)$ & D (0.99) & $\mathrm{D}(0.99)$ \\
\hline $\mathrm{D}(\mathrm{l})$ & $\mathrm{D}(1)$ & D (0.99) & $\mathrm{D}(1)$ & $\mathrm{D}(1)$ & $\mathrm{D}(1)$ & $\mathrm{D}(1)$ & $\mathrm{D}(1)$ & $\mathrm{D}(1)$ \\
\hline$\frac{-421}{4.079}$ & $\begin{array}{l}\frac{4.44}{5.129} \\
5\end{array}$ & $\begin{array}{l}\frac{3.82}{3.987} \\
3.987\end{array}$ & $\begin{array}{l}\frac{3.82}{4.806} \\
4\end{array}$ & $\begin{array}{l}\frac{3.82}{4.806} \\
4.80\end{array}$ & $\frac{3.71}{4.725}$ & $\begin{array}{l}\frac{3.71}{4.725} \\
4\end{array}$ & $\begin{array}{l}3.71 \\
3.918 \\
\end{array}$ & $\begin{array}{l}3.71 \\
4.725 \\
\end{array}$ \\
\hline 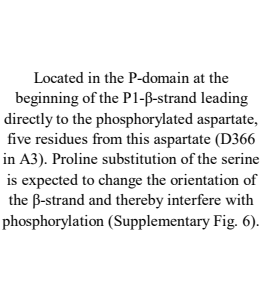 & 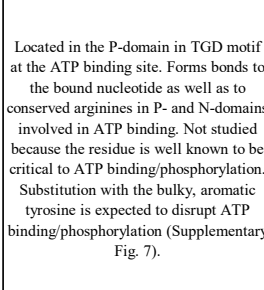 & 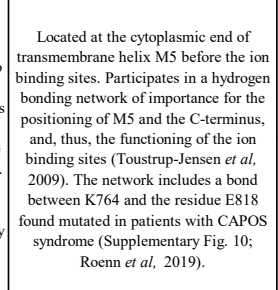 & 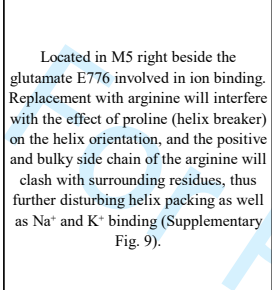 & 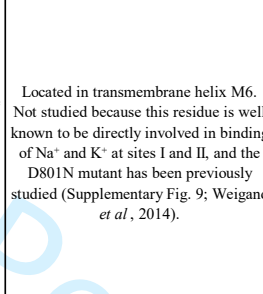 & \multicolumn{2}{|c|}{ 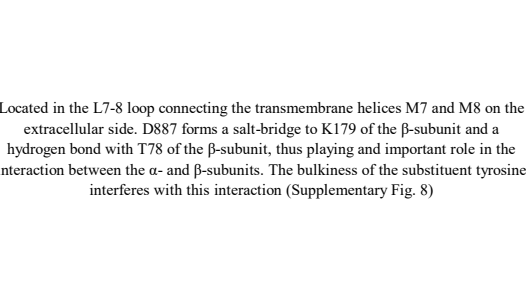 } & 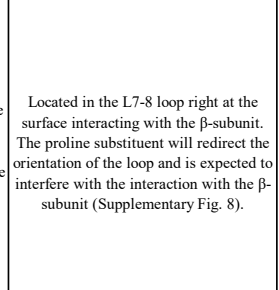 & 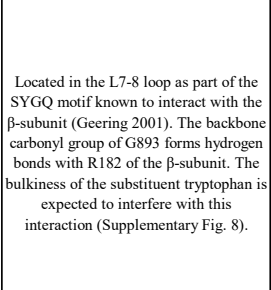 \\
\hline YES (this study) & No & YES (his study) & YES (this study) & YES (Weignd etal, 2014). & \multicolumn{2}{|c|}{ YES (his study) } & YES (this study) & No \\
\hline No & $\mathrm{NA}$ & No & No & $\mathrm{NA}$ & \multirow{2}{*}{\multicolumn{2}{|c|}{ 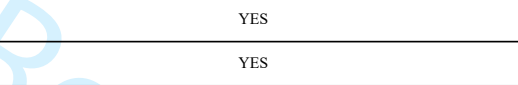 }} & мо & $\mathrm{NA}$ \\
\hline № & $\mathrm{NA}$ & (YES) & № & $\mathrm{NA}$ & & & № & $\mathrm{NA}$ \\
\hline 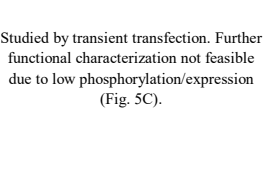 & $\mathrm{NA}$ & 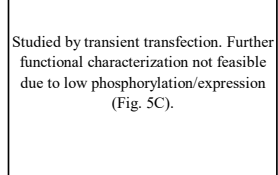 & 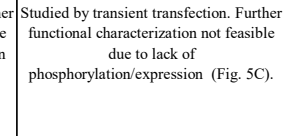 & 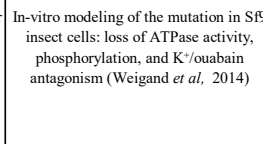 & \multicolumn{2}{|c|}{ 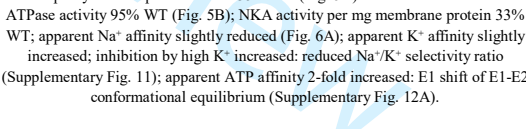 } & 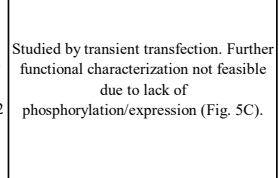 & $\mathrm{NA}$ \\
\hline 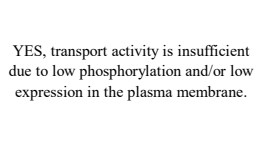 & 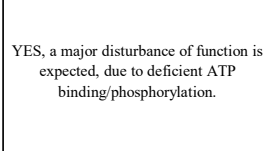 & 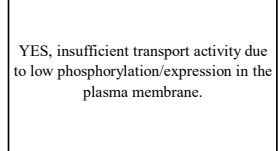 & 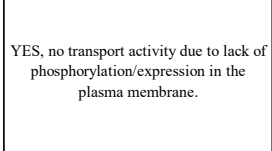 & 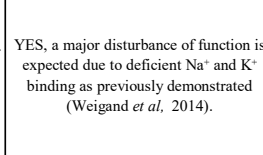 & 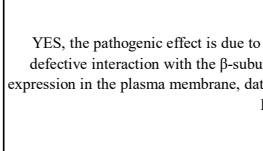 & 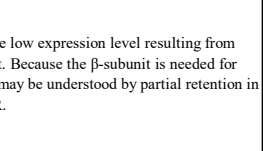 & 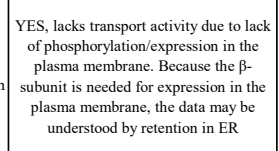 & 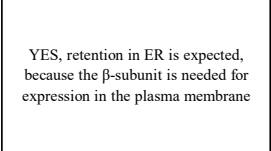 \\
\hline Novel & 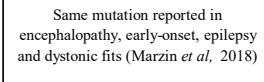 & Novel & Novel & 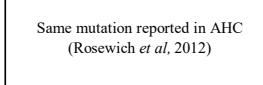 & & & 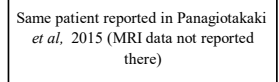 & 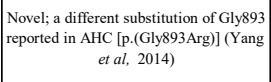 \\
\hline $\mathrm{NR}$ & $\mathrm{NR}$ & NR & NR & $\mathrm{NR}$ & $\mathrm{NR}$ & $\mathrm{NR}$ & $\mathrm{NR}$ & \begin{tabular}{|c|c|} 
A2-G900R reported in HM (Depreze \\
$a l, 2008)$
\end{tabular} \\
\hline
\end{tabular}




\begin{tabular}{|c|c|c|c|c|c|}
\hline \multicolumn{5}{|c|}{$A T P P A 3$, NM_152296.4, heteroroggous } & ATPIIL, NM__0007023,3, homorygous \\
\hline 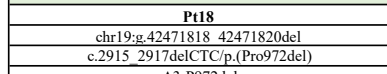 & 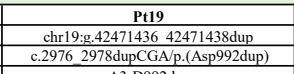 & 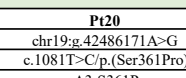 & 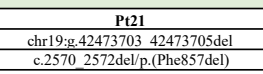 & 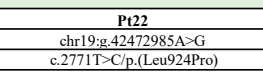 & 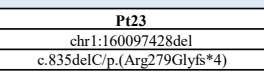 \\
\hline 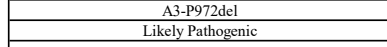 & \begin{tabular}{|c|} 
A-1-3992dup \\
Pathogenic \\
\end{tabular} & $\begin{array}{c}\text { A-3.3319 } \\
\text { Pathogenic } \\
\end{array}$ & 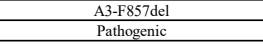 & $\begin{array}{c}\frac{A 3-1.292 \mathrm{P}}{2} \text { Pathognic } \\
\end{array}$ & $\begin{array}{c}\text { A2-2R79GGS } \\
\text { Pathogenic } \\
\end{array}$ \\
\hline 21.4 & 33 & 25.8 & 18.62 & 29.4 & 32 \\
\hline $\mathrm{NA}$ & $\mathrm{NA}$ & D (0) & $\mathrm{NA}$ & D (0) & $\mathrm{NA}$ \\
\hline $\mathrm{NA}$ & $\mathrm{NA}$ & $\mathrm{P}(0.86)$ & $\mathrm{NA}$ & $\mathrm{D}(0.97)$ & $\mathrm{NA}$ \\
\hline $\mathrm{D}(1)$ & $\mathrm{D}(0.99)$ & $\mathrm{D}(1)$ & $\mathrm{D}(0.99)$ & $\mathrm{D}(1)$ & $\mathrm{D}(1)$ \\
\hline$\frac{3.27}{4.551}$ & $\begin{array}{l}3.32 \\
3.722 \\
\end{array}$ & $\frac{4.21}{4.145}$ & $\begin{array}{l}\frac{3.35}{3.95} \\
3.95-10\end{array}$ & $\begin{array}{l}\frac{3.42}{3.82} \\
3.82-10\end{array}$ & $\begin{array}{l}4.89 \\
3.688 \\
\end{array}$ \\
\hline 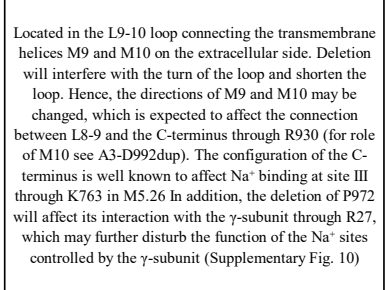 & 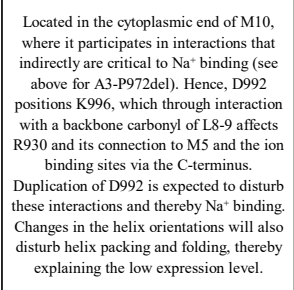 & Same as Patient 9 & 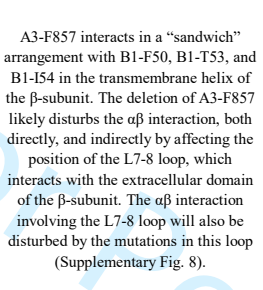 & 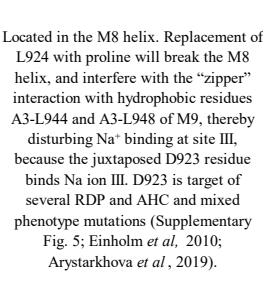 & 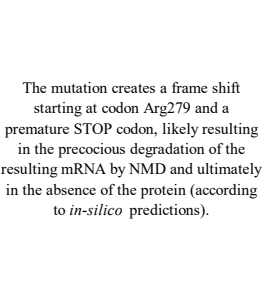 \\
\hline YES (this study) & YES (this study) & YES (this study) & No & YES (Arsstarkhovora tal, 2019) & No \\
\hline YES & No & No & $\mathrm{NA}$ & YES & $\mathrm{NA}$ \\
\hline YES & No & No & $\mathrm{NA}$ & $\mathrm{NA}$ & $\mathrm{NA}$ \\
\hline 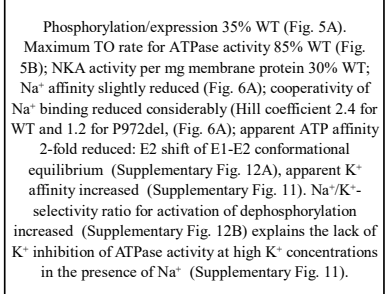 & 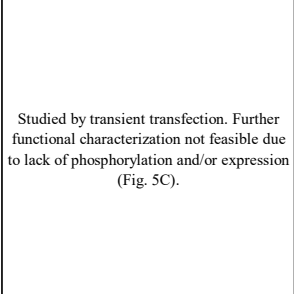 & Same as Patien 9 & $\mathrm{NA}$ & 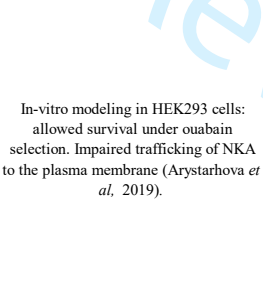 & \\
\hline 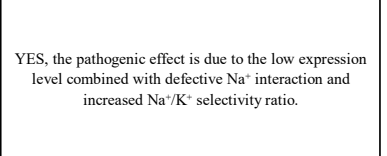 & 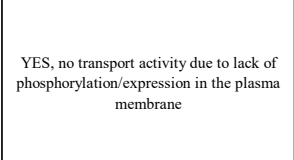 & Same es Patien 9 & 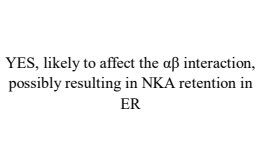 & 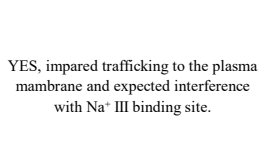 & 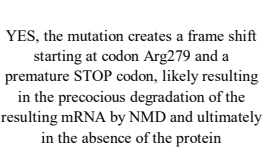 \\
\hline Novel & 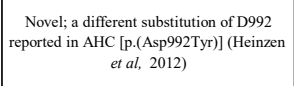 & Novel & 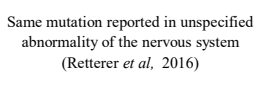 & 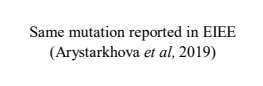 & $\begin{array}{l}\text { Same patient as } \mathrm{PP} \text { i in Monteirio e elal, } \\
2020\end{array}$ \\
\hline A2-P979 Leported in HM (Jurkat-Rotte tal, 2004) & \begin{tabular}{|l|} 
A2-D999H reported in HM(Fermandeze \\
all 2008)
\end{tabular} & NR & $\mathrm{NR}$ & NR & NR \\
\hline
\end{tabular}


Supplementary Table 1 - Genomic coordinates, ACMG classification, in-silico predictions, structural and (where available) in-vitro functional consequences of the mutations in our cohort. The ability of NKA to support cell growth in stable transfection and to phosphorylate from ATP are reported as evaluated in this study in a COS-1 cellular model. Transient transfection in the presence of siRNA to knock down the endogenous NKA was applied to study those mutations not supporting cell growth in stable transfections. $\S$ Supporting cell growth in stable transfection requires at least 5-10\% of NKA transport activity; \# NKA phosphorylation requires expression in cell membrane as well as ATP and Na binding. The results of in-silico prediction from SIFT, PolyPhen-2, and Mutation Taster, as well as the conservation scores from GERP++ and PhyloP are showed. Confidence score for each prediction tool is reported in brackets: SIFT score for a missense variant is computed as (1-p) where $\mathrm{p}$ is the probability for the variant to be deleterious. For Mutation Taster a value close to 1 indicates a high 'security' of the prediction, while forPolyPhen2 it represents the probability for the variant to be disease causing (in the 0-1 range). GERP++ and PhyloP scores range from -12.3 to 6.17, and from -14.1 to 6.4, respectively, with higher scores indicating stronger constraint. Abbreviations: AHC, alternating hemiplegia of childhood; ER, endoplasmic reticulum; HM, hemiplegic migraine; NMD, nonsense-mediated mRNA decay; D, damaging; P, possibly damaging; NA, not available; NR, not reported; TO, turnover. 


\section{Appendix: ATP1A2/A3-collaborators}

Damien Sanlaville ${ }^{1}$, Rani Sachdev ${ }^{2,3}$, Ian Andrews ${ }^{2,3}$, Francesco Mari ${ }^{4}$, Anna Cavalli ${ }^{4}$, Carmen Barba ${ }^{4}$, Beatrice De Maria ${ }^{4}$, Giampaolo Garani ${ }^{5}$, Johannes R. Lemke ${ }^{6}$, Mario Mastrangelo ${ }^{7}$, Emily Tam ${ }^{8}$, Elizabeth Donner ${ }^{8}$, Helen Branson ${ }^{9}$, Fabiola P. Monteiro ${ }^{10}$, Fernando Kok ${ }^{10}$, Katherine B Howell ${ }^{11}$, Stephanie Leech ${ }^{12}$, Heather Mefford $^{13}$, Alison Muir ${ }^{13}$

1. Department of Medical Genetics, Member of the ERN EpiCARE, University Hospital of Lyon, Lyon, France

2. Centre for Clinical Genetics, Sydney Children's Hospital, Randwick, NSW, Australia

3. School of Women's and Children's Health, University of New South Wales, Randwick, NSW, Australia

4. Pediatric Neurology, Neurogenetics and Neurobiology Unit and Laboratories, Meyer Children's Hospital, University of Florence, Florence, Italy

5. Neonatal Intensive Care Unit, Department of Mother and Child, Ferrara University Hospital, Ferrara, Italy

6. Institute of Human Genetics, University of Leipzig Hospitals and Clinics, Leipzig, Germany

7. Department of Human Neuroscience, Unit of Child Neurology and Psychiatry, Sapienza University, Rome, Italy

8. Division of Neurology, Department of Pediatrics, The Hospital for Sick Children, Toronto, Ontario, Canada

9. Diagnostic Imaging, The Hospital for Sick Children, Toronto, Ontario, Canada

10. Mendelics Genomic Analysis, CEP 04013-000, São Paulo, SP, Brazil

11. Murdoch Children's Research Institute, Royal Children's Hospital, Melbourne, Australia

12. University of Melbourne, Australia

13. Department of Pediatrics, Division of Genetic Medicine, University of Washington, Seattle, WA 


\section{Supplementary References}

Arystarkhova E, Haq IU, Luebbert T, Mochel F, Saunders-Pullman R, Bressman SB, et al. Factors in the disease severity of ATP1A3 mutations: Impairment, misfolding, and allele competition. Neurobiol Dis 2019; 132: 104577.

Baginski ES, Foà PP, Zak B. Microdetermination of inorganic phosphate, phospholipids, and total phosphate in biologic materials. Clin Chem 1967; 13: 326-32.

Cooper GM, Stone EA, Asimenos G, Green ED, Batzoglou S, Sidow A. Distribution and intensity of constraint in mammalian genomic sequence. Genome Res 2005; 15: 901-13.

Deprez L, Weckhuysen S, Peeters K, Deconinck T, Claeys KG, Claes LRF, et al. Epilepsy as part of the phenotype associated with ATP1A2 mutations. Epilepsia 2008; 49: 500-8.

DePristo MA, Banks E, Poplin R, Garimella K V., Maguire JR, Hartl C, et al. A framework for variation discovery and genotyping using next-generation DNA sequencing data. Nat Genet 2011; 43: 491-8.

Einholm AP, Nielsen HN, Holm R, Toustrup-Jensen MS, Vilsen B. Importance of a potential protein kinase a phosphorylation site of $\mathrm{Na}+, \mathrm{K}+-\mathrm{ATPase}$ and its interaction network for $\mathrm{Na}+$ binding. J Biol Chem 2016; 291 : $10934-47$.

Einholm AP, Toustrup-Jensen MS, Holm R, Andersen JP, Vilsen B. The rapid-onset dystonia parkinsonism mutation $\mathrm{D} 923 \mathrm{~N}$ of the $\mathrm{Na}+, \mathrm{K}+$-ATPase $\alpha 3$ isoform disrupts $\mathrm{Na}+$ interaction at the third $\mathrm{Na}+$ site. $\mathrm{J}$ Biol Chem 2010; $285: 26245-54$.

Fernandez DM, Hand CK, Sweeney BJ, Parfrey NA. A novel ATP1A2 gene mutation in an Irish familial hemiplegic migraine kindred. Headache 2008; 48: 101-8.

Geering K. The functional role of $\beta$ subunits in oligomeric P-type ATPases. J Bioenerg Biomembr 2001; 33: $425-38$.

Guerrini R, Dobyns WB. Malformations of cortical development: Clinical features and genetic causes. Lancet Neurol 2014; 13: 710-26.

Heinzen EL, Swoboda KJ, Hitomi Y, Gurrieri F, De Vries B, Tiziano FD, et al. De novo mutations in ATP1A3 cause alternating hemiplegia of childhood. Nat Genet 2012; 44: 1030-4.

Holm R, Toustrup-Jensen MS, Einholm AP, Schack VR, Andersen JP, Vilsen B. Neurological disease mutations of $\alpha 3 \mathrm{Na}+, \mathrm{K}+-\mathrm{ATPase}$ : Structural and functional perspectives and rescue of compromised function. Biochim Biophys Acta - Bioenerg 2016; 1857: 1807-28.

Jagadeesh KA, Wenger AM, Berger MJ, Guturu H, Stenson PD, Cooper DN, et al. M-CAP eliminates a majority of variants of uncertain significance in clinical exomes at high sensitivity. Nat Genet 2016; 48: 1581-6.

Jurkat-Rott K, Freilinger T, Dreier JP, Herzog J, Göbel H, Petzold GC, et al. Variability of familial hemiplegic migraine with novel A1A2 Na +/K+-ATPase variants. Neurology 2004; 62: 1857-61. 
Kanai R, Ogawa H, Vilsen B, Cornelius F, Toyoshima C. Crystal structure of a Na + -bound Na,$+ \mathrm{K}+$-ATPase preceding the E1P state. Nature 2013; 502: 201-6.

Li H, Durbin R. Fast and accurate short read alignment with Burrows-Wheeler transform. Bioinformatics 2009; 25: 1754-60.

Liu X, Jian X, Boerwinkle E. dbNSFP: a lightweight database of human nonsynonymous SNPs and their functional predictions. Hum Mutat 2011; 32: 894-9.

Marzin P, Mignot C, Dorison N, Dufour L, Ville D, Kaminska A, et al. Early-onset encephalopathy with paroxysmal movement disorders and epileptic seizures without hemiplegic attacks: About three children with novel ATP1A3 mutations. Brain Dev 2018; 40: 768-74.

Møller RS, Dahl HA, Helbig I. The contribution of next generation sequencing to epilepsy genetics. Expert Rev Mol Diagn 2015; 15: 1531-8.

Monteiro FP, Curry CJ, Hevner R, Elliott S, Fisher JH, Turocy J, et al. Biallelic loss of function variants in ATP1A2 cause hydrops fetalis, microcephaly, arthrogryposis and extensive cortical malformations. Eur J Med Genet 2020; 63: 103624 .

Nielsen HN, Spontarelli K, Holm R, Andersen JP, Einholm AP, Artigas P, et al. Distinct effects of Q925 mutation on intracellular and extracellular $\mathrm{Na}+$ and $\mathrm{K}+$ binding to the $\mathrm{Na}+, \mathrm{K}+$-ATPase. Sci Rep 2019; 9

Paciorkowski AR, McDaniel SS, Jansen LA, Tully H, Tuttle E, Ghoneim DH, et al. Novel mutations in ATP1A3 associated with catastrophic early life epilepsy, episodic prolonged apnea, and postnatal microcephaly. Epilepsia $2015 ; 56: 422-30$.

Palmer EE, Schofield D, Shrestha R, Kandula T, Macintosh R, Lawson JA, et al. Integrating exome sequencing into a diagnostic pathway for epileptic encephalopathy: Evidence of clinical utility and cost effectiveness. Mol Genet genomic Med 2018; 6: 186-99.

Panagiotakaki E, de Grandis E, Stagnaro M, Heinzen EL, Fons C, Sisodiya S, et al. Clinical profile of patients with ATP1A3 mutations in Alternating Hemiplegia of Childhood - A study of 155 patients. Orphanet J Rare Dis $2015 ; 10$

Parrini E, Marini C, Mei D, Galuppi A, Cellini E, Pucatti D, et al. Diagnostic Targeted Resequencing in 349 Patients with Drug-Resistant Pediatric Epilepsies Identifies Causative Mutations in 30 Different Genes. Hum Mutat 2017; 38: 216-25.

Pollard KS, Hubisz MJ, Rosenbloom KR, Siepel A. Detection of nonneutral substitution rates on mammalian phylogenies. Genome Res 2010; 20: 110-21.

Rentzsch P, Witten D, Cooper GM, Shendure J, Kircher M. CADD: predicting the deleteriousness of variants throughout the human genome. Nucleic Acids Res 2019; 47: D886-94.

Retterer K, Juusola J, Cho MT, Vitazka P, Millan F, Gibellini F, et al. Clinical application of whole-exome sequencing across clinical indications. Genet Med 2016; 18: 696-704. 
Richards S, Aziz N, Bale S, Bick D, Das S, Gastier-Foster J, et al. Standards and guidelines for the interpretation of sequence variants: A joint consensus recommendation of the American College of Medical Genetics and Genomics and the Association for Molecular Pathology. Genet Med 2015; 17

Roenn CP, Li M, Schack VR, Forster IC, Holm R, Toustrup-Jensen MS, et al. Functional consequences of the CAPOS mutation E818K of Na,K-ATPase. J Biol Chem 2019; 294: 269-80.

Rosewich H, Thiele H, Ohlenbusch A, Maschke U, Altmüller J, Frommolt P, et al. Heterozygous de-novo mutations in ATP1A3 in patients with alternating hemiplegia of childhood: A whole-exome sequencing geneidentification study. Lancet Neurol 2012; 11: 764-73.

Sasaki M, Ishii A, Saito Y, Morisada N, Iijima K, Takada S, et al. Genotype-phenotype correlations in alternating hemiplegia of childhood. Neurology 2014; 82: 482-90.

Schack VR, Holm R, Vilsen B. Inhibition of phosphorylation of $\mathrm{Na}+, \mathrm{K}+$-ATPase by mutations causing familial hemiplegic migraine. J Biol Chem 2012; 287: 2191-202.

Schrödinger. PyMOL, molecular visualization system. Available at https://pymol.org 2018

Schwarz JM, Rödelsperger C, Schuelke M, Seelow D. MutationTaster evaluates disease-causing potential of sequence alterations. Nat Methods 2010; 7: 575-6.

Shinoda T, Ogawa H, Cornelius F, Toyoshima C. Crystal structure of the sodium-potassium pump at 2.4 resolution. Nature 2009; 459: 446-50.

Sievers F, Wilm A, Dineen D, Gibson TJ, Karplus K, Li W, et al. Fast, scalable generation of high-quality protein multiple sequence alignments using Clustal Omega. Mol Syst Biol 2011; 7

Stenson PD, Mort M, Ball E V., Evans K, Hayden M, Heywood S, et al. The Human Gene Mutation Database: towards a comprehensive repository of inherited mutation data for medical research, genetic diagnosis and nextgeneration sequencing studies. Hum Genet 2017; 136: 665-77.

Sweadner KJ, Toro C, Whitlow CT, Snively BM, Cook JF, Ozelius LJ, et al. ATP1A3 mutation in adult rapidonset ataxia. PLoS One 2016; 11: 1-12.

Tavraz NN, Dürr KL, Koenderink JB, Freilinger T, Bamberg E, Dichgans M, et al. Impaired plasma membrane targeting or protein stability by certain ATP1A2 mutations identified in sporadic or familial hemiplegic migraine. Channels 2009; 3: 82-7.

Thorvaldsdóttir H, Robinson JT, Mesirov JP. Integrative Genomics Viewer (IGV): high-performance genomics data visualization and exploration. Brief Bioinform 2013; 14: 178-92.

Toustrup-Jensen MS, Einholm AP, Schack VR, Nielsen HN, Holm R, Sobrido MJ, et al. Relationship between Intracellular $\mathrm{Na}+$ Concentration and Reduced $\mathrm{Na}+$ Affinity in $\mathrm{Na}+, \mathrm{K}+$-ATPase Mutants causing Neurological Disease. J Biol Chem 2014; 289: 3186-97.

Toustrup-Jensen MS, Holm R, Einholm VR, Morth PJ, Nissen P, Andersen JP, et al. The C terminus of Na,$+ \mathrm{K}$ + -ATPase controls $\mathrm{Na}+$ affinity on both sides of the membrane through Arg 935. J Biol Chem 2009; 284 : 
$18715-25$.

Ueda K, Serajee F, Huq AM. Clinical benefit of NMDA receptor antagonists in a patient with ATP1A2 gene mutation. Pediatrics 2018; 141: S390-4.

Vanmolkot KRJ, Kors EE, Turk U, Turkdogan D, Keyser A, Broos LAM, et al. Two de novo mutations in the $\mathrm{Na}, \mathrm{K}-\mathrm{ATP}$ ase gene ATP1A2 associated with pure familial hemiplegic migraine. Eur J Hum Genet 2006; 14 : $555-60$.

Viollet L, Glusman G, Murphy KJ, Newcomb TM, Reyna SP, Sweney M, et al. Alternating hemiplegia of childhood: Retrospective genetic study and genotype-phenotype correlations in 187 subjects from the US AHCF registry. PLoS One 2015; 10: 1-14.

De Vries B, Freilinger T, Vanmolkot KRJ, Koenderink JB, Stam AH, Terwindt GM, et al. Systematic analysis of three FHM genes in 39 sporadic patients with hemiplegic migraine. Neurology 2007; 69: 2170-6.

Weigand KM, Messchaert M, Swarts HGP, Russel FGM, Koenderink JB. Alternating Hemiplegia of Childhood mutations have a differential effect on $\mathrm{Na}^{+}, \mathrm{K}+-$-ATPase activity and ouabain binding. Biochim Biophys Acta Mol Basis Dis 2014; 1842: 1010-6.

Yang X, Gao H, Zhang J, Xu X, Liu X, Wu X, et al. ATP1A3 mutations and genotype-phenotype correlation of alternating hemiplegia of childhood in chinese patients. PLoS One 2014; 9 
STREGA statement: Reporting guidelines checklist for genetic association studies

\begin{tabular}{|c|c|c|c|}
\hline SECTION & \begin{tabular}{|l|} 
ITEM \\
NUMBER
\end{tabular} & CHECKLIST ITEM & \begin{tabular}{|l|} 
REPORTED ON \\
PAGE NUMBER:
\end{tabular} \\
\hline \multicolumn{4}{|l|}{ TITLE AND ABSTRACT } \\
\hline & $1 \mathrm{a}$ & Indicate the study's design with a commonly used term in the title or the abstract & 1 and 4 \\
\hline & $1 b$ & $\begin{array}{l}\text { Provide in the abstract an informative and balanced summary of what was done and what } \\
\text { was found }\end{array}$ & 4 \\
\hline \multicolumn{4}{|l|}{ INTRODUCTION } \\
\hline \multirow{2}{*}{ Background and objectives } & 2 & Explain the scientific background and rationale for the investigation being reported & $6-7$ \\
\hline & 3 & $\begin{array}{l}\text { State specific objectives, including any pre-specified hypotheses. State if the study is the } \\
\text { first report of a genetic association, a replication effort, or both }\end{array}$ & $6-7$ \\
\hline \multicolumn{4}{|l|}{ METHODS } \\
\hline Study design & 4 & Present key elements of study design early in the paper & $7-8$ \\
\hline Setting & 5 & $\begin{array}{l}\text { Describe the setting, locations, and relevant dates, including periods of recruitment, } \\
\text { exposure, follow-up, and data collection }\end{array}$ & 7-8 \\
\hline Participants & $6 a$ & $\begin{array}{l}\text { Give information on the criteria and methods for selection of subsets of participants from a } \\
\text { larger study, when relevant }\end{array}$ & 7-8 \\
\hline Variables & 7 & $\begin{array}{l}\text { Clearly define genetic exposures (genetic variants) using a widely-used nomenclature } \\
\text { system. Identify variables likely to be associated with population stratification (confounding } \\
\text { by ethnic origin) }\end{array}$ & 7-8 \\
\hline $\begin{array}{l}\text { Data } \\
\text { sources/measurements }\end{array}$ & $8^{*}$ & $\begin{array}{l}\text { Describe laboratory methods, including source and storage of DNA, genotyping methods } \\
\text { and platforms (including the allele calling algorithm used, and its version), error rates and } \\
\text { call rates. State the laboratory/centre where genotyping was done. Describe comparability } \\
\text { of laboratory methods if there is more than one group. Specify whether genotypes were } \\
\text { assigned using all of the data from the study simultaneously or in smaller batches }\end{array}$ & 7-8 \\
\hline Bias & 9 & $\begin{array}{l}\text { For quantitative outcome variables, specify if any investigation of potential bias resulting } \\
\text { from pharmacotherapy was undertaken. If relevant, describe the nature and magnitude of } \\
\text { the potential bias, and explain what approach was used to deal with this }\end{array}$ & NA \\
\hline
\end{tabular}




\begin{tabular}{|c|c|c|c|}
\hline SECTION & $\begin{array}{l}\text { ITEM } \\
\text { NUMBER }\end{array}$ & CHECKLIST ITEM & $\begin{array}{l}\text { REPORTED ON } \\
\text { PAGE NUMBER: } \\
\end{array}$ \\
\hline Study size & 10 & Explain how the study size was arrived at & $7-8$ \\
\hline Quantitative variables & 11 & If applicable, describe how effects of treatment were dealt with & NA \\
\hline \multirow[t]{10}{*}{ Statistical methods } & $12 a$ & $\begin{array}{l}\text { Describe all statistical methods, including those used to control for confounding. State } \\
\text { software version used and options (or settings) chosen }\end{array}$ & NA \\
\hline & $12 \mathrm{~b}$ & Describe any methods used to examine subgroups and interactions & NA \\
\hline & $12 \mathrm{c}$ & Explain how missing data were addressed & NA \\
\hline & $12 d$ & $\begin{array}{l}\text { Cohort study-If applicable, explain how loss to follow-up was addressed } \\
\text { Case-control study-If applicable, explain how matching of cases and controls was } \\
\text { addressed } \\
\text { Cross-sectional study-If applicable, describe analytical methods taking account of } \\
\text { sampling strategy }\end{array}$ & NA \\
\hline & $12 \mathrm{e}$ & Describe any sensitivity analyses & NA \\
\hline & $12 \mathrm{f}$ & State whether Hardy-Weinberg equilibrium was considered and, if so, how & NA \\
\hline & $12 \mathrm{~g}$ & Describe any methods used for inferring genotypes or haplotypes & $7-8$ \\
\hline & $12 \mathrm{~h}$ & Describe any methods used to assess or address population stratification & NA \\
\hline & $12 \mathrm{i}$ & $\begin{array}{l}\text { Describe any methods used to address multiple comparisons or to control risk of false- } \\
\text { positive findings }\end{array}$ & NA \\
\hline & $12 \mathrm{j}$ & Describe any methods used to address and correct for relatedness among subjects & $7-8$ \\
\hline \multicolumn{4}{|l|}{ RESULTS } \\
\hline \multirow[t]{3}{*}{ Participants } & $13 a$ & $\begin{array}{l}\text { Report numbers of individuals in whom genotyping was attempted and numbers of } \\
\text { individuals in whom genotyping was successful }\end{array}$ & 8 \\
\hline & $13 b$ & Give reasons for non-participation at each stage & NA \\
\hline & $13 c$ & Consider use of a flow diagram & NA \\
\hline
\end{tabular}




\begin{tabular}{|c|c|c|c|}
\hline SECTION & $\begin{array}{l}\text { ITEM } \\
\text { NUMBER }\end{array}$ & CHECKLIST ITEM & $\begin{array}{l}\text { REPORTED ON } \\
\text { PAGE NUMBER }\end{array}$ \\
\hline \multirow[t]{3}{*}{ Descriptive Data } & $14 a$ & $\begin{array}{l}\text { Give characteristics of study participants (e.g. demographic, clinical, social) and information } \\
\text { on exposures and potential confounders. Consider giving information by genotype }\end{array}$ & 8 \\
\hline & $14 \mathrm{~b}$ & Indicate number of participants with missing data for each variable of interest & NA \\
\hline & $14 \mathrm{c}$ & Cohort study-Summarise follow-up time (eg, average and total amount) & NA \\
\hline Outcome Data & $15^{*}$ & $\begin{array}{l}\text { Cohort study- Report outcomes (phenotypes) for each genotype category over time } \\
\text { Case-control study- Report numbers in each genotype category } \\
\text { Cross-sectional study- Report outcomes (phenotypes) for each genotype category }\end{array}$ & $8-10$ \\
\hline \multirow[t]{4}{*}{ Main Results } & $16 a$ & $\begin{array}{l}\text { Give unadjusted estimates and, if applicable, confounder-adjusted estimates and their } \\
\text { precision (e.g. } 95 \% \text { confidence interval). Make clear which confounders were adjusted for } \\
\text { and why they were included }\end{array}$ & NA \\
\hline & $16 \mathrm{~b}$ & Report category boundaries when continuous variables were categorized & NA \\
\hline & $16 \mathrm{c}$ & $\begin{array}{l}\text { If relevant, consider translating estimates of relative risk into absolute risk for a meaningful } \\
\text { time period }\end{array}$ & NA \\
\hline & $16 \mathrm{~d}$ & Report results of any adjustments for multiple comparisons & NA \\
\hline \multirow[t]{3}{*}{ Other Analyses } & $17 a$ & $\begin{array}{l}\text { Report other analyses done-e.g. analyses of subgroups and interactions, and sensitivity } \\
\text { analyses }\end{array}$ & NA \\
\hline & $17 \mathrm{~b}$ & $\begin{array}{l}\text { If numerous genetic exposures (genetic variants) were examined, summarize results from } \\
\text { all analyses undertaken }\end{array}$ & NA \\
\hline & $17 \mathrm{c}$ & If detailed results are available elsewhere, state how they can be accessed & NA \\
\hline \multicolumn{4}{|l|}{ DISCUSSION } \\
\hline Key Results & 18 & Summarise key results with reference to study objectives & $14-21$ \\
\hline Limitations & 19 & $\begin{array}{l}\text { Discuss limitations of the study, taking into account sources of potential bias or } \\
\text { imprecision. Discuss both direction and magnitude of any potential bias }\end{array}$ & $14-21$ \\
\hline Interpretation & 20 & $\begin{array}{l}\text { Give a cautious overall interpretation of results considering objectives, limitations, } \\
\text { multiplicity of analyses, results from similar studies, and other relevant evidence }\end{array}$ & $14-21$ \\
\hline Generalisability & 21 & $\begin{array}{l}\text { Discuss the generalisability (external validity) of the study results } \\
\text { Other information }\end{array}$ & $14-21$ \\
\hline FUNDING & & & \\
\hline
\end{tabular}




\begin{tabular}{|l|l|l|l|}
\hline SECTION & $\begin{array}{l}\text { ITEM } \\
\text { NUMBER }\end{array}$ & CHECKLIST ITEM & $\begin{array}{l}\text { REPORTED ON } \\
\text { PAGE NUMBER: }\end{array}$ \\
\hline & 22 & $\begin{array}{l}\text { Give the source of funding and the role of the funders for the present study and, if } \\
\text { applicable, for the original study on which the present article is based }\end{array}$ & 22 \\
\hline & & & \\
\hline
\end{tabular}

*Give information separately for cases and controls in case-control studies and, if applicable, for exposed and unexposed groups in cohort and crosssectional studies.

Reference: von Elm E, Altman DG, Egger M, Pocock SJ, Gotzsche PC, Vandenbroucke JP. The Strengthening the Reporting of Observational Studies in Epidemiology (STROBE) Statement: guidelines for reporting observational studies. This guideline was published simultaneously in 8 journals.

Ann Intern Med. 2007; 147(8):573-577. PMID: 17938396, PLoS Med. 2007;4(10):e296. PMID: 17941714, BMJ. 2007;335(7624):806-808. PMID: 17947786, Prev Med. 2007;45(4):247-251. PMID: 17950122, Epidemiology. 2007;18(6):800-804. PMID: 18049194, Lancet. 2007;370(9596):1453-1457. PMID: 18064739 J Clin Epidemiol. 2008;61(4):344-349. PMID: 18313558, Bull World Health Organ. 2007;85(11):867-872. PMID: 18038077 